\title{
THE POWER OF “ALTRUISTIC" HEALTHCARE AND ITS IMPACTS ON THE HEALTH-SEEKING BEHAVIOUR OF KOREAN IMMIGRANTS IN NEW ZEALAND
}

\author{
BY \\ MIKYUNG KIM
}

\begin{abstract}
A thesis
submitted to the Victoria University of Wellington

in fulfilment of the requirements for the degree of

Doctor of Philosophy in Health
\end{abstract}

Victoria University of Wellington

2016 



\begin{abstract}
Immigrants' health-seeking behaviours are reconstructed during their healthcare adapting process as a consequence of interaction with the host health services. How Korean immigrants, who came from a very different healthcare system, reconstructed their health-seeking behaviours by adapting in New Zealand, and the factors affecting their healthcare decisions and behaviours were the main concerns of the research. Korean immigrants' experiences were explored and theorised in order to provide a theoretical understanding of their health-seeking behaviours. Glaser's classic grounded theory was used to generate a conceptual theory with emergent fit. Forty-eight participants were recruited from Wellington and Auckland. The primary data were collected from unstructured face-to-face individual interviews ( $\mathrm{n}=23)$, seven two-person interviews ( $\mathrm{n}=14$ [two people attended twice]), and four group interviews ( $\mathrm{n}=14$ [one person attended twice]). In order to develop a theory grounded in the participants' experiences and perspectives, data were collected, coded and analysed congruently and iteratively with the constant comparison and theoretical sampling methods.
\end{abstract}

The Healthcare Acculturation Theory that emerged from this research elucidated Koreans' healthcare adapting process and provided insights into their health-seeking behaviours in New Zealand. Koreans' health-seeking behaviours were largely categorised into four positions: "rejecting", "attuning", "attuning but negotiating", and "rejecting but negotiating". They were found to be in one of the four positions at any one point in time and each position reflected their responses toward the New Zealand healthcare system. Once a specific position was held, their healthcare decisions and behaviours were directed by the said position. As their healthcare experiences were ongoing, their chosen positions were not end-outcomes in themselves. When their existing perceptions and behaviours were challenged by new experiences their healthcare positions transitioned or reinforced, and these changes informed the Healthcare Acculturation Theory.

When Korean immigrants, who came from a commercially driven healthcare system, experienced healthcare (emergency care rather than elective) at the public 
services in New Zealand they were deeply touched. The healthcare they received was caring, empathetic, and responsive care delivered in a person-centred way that they had never experienced before. This experience rendered a turning point in their healthcare adapting process. Participants who reached such a point experienced transitions, not only in their healthcare positions, but in their life beliefs and values as well, which went far beyond simply a shifting perspective but can be likened more to a "conversion". It was conversion to a new understanding. This research articulates the major contributing factor to immigrants' acceptance of healthcare, which was receiving care that Koreans perceived as "altruistic". It is related to the ambience of health services that resulted in sincere and empathetic care, rather than the barriers to healthcare that immigrants face in the host country. This thesis makes an important contribution to the existing body of knowledge on acculturation demonstrating new knowledge and a theoretical understanding of health-seeking behaviour, in the development of a substantive theory based on the experiences of Korean immigrants in New Zealand.

Key words: health-seeking behaviour, health service utilisation, quality of health service, immigrants, acculturation, patient satisfaction, person-centred care 


\section{Acknowledgements}

I would like to express my sincere gratitude to all those who supported my $\mathrm{PhD}$ journey over the past three years. The completion of this thesis would not have occurred without their support. I could do this through God who gave me strength throughout the journey.

First, my appreciation goes to the Victoria Doctoral Scholarship for giving me the opportunity to study at Victoria University of Wellington. I would like to acknowledge my thankfulness to the Korean immigrants who participated in this thesis by putting in time and sharing their experiences and life stories. I am extremely grateful to my supervisors, Dr Kay de Vries and Dr Kathy Nelson. Your guidance and support with persistent encouragement enabled me to accomplish this project. I enjoyed the supervision meetings with you and have learned from your extensive knowledge and expertise, and through your critiques and advice. It was a very precious time for me and I appreciate that. Having you as my supervisors was a great blessing to me. I must also thank all of my friends, colleagues, and the staff at the Graduate School of Nursing, Midwifery and Health for their support. To my husband and my best friend, Ashley, my deepest thanks for your constant love and caring support during the study. I would never have been able to study abroad without you. Thank you for always being there for me. I dedicate this thesis to my husband with love and respect. 


\section{Table of contents}

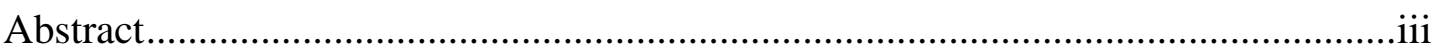

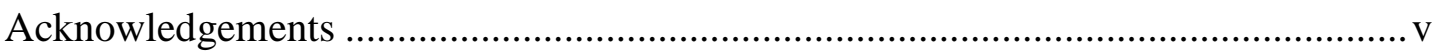

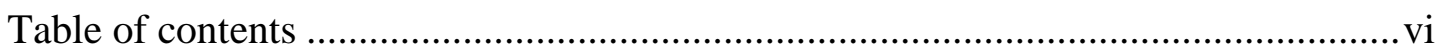

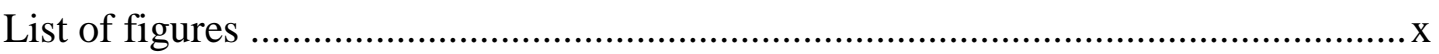

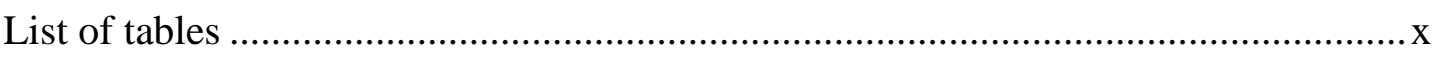

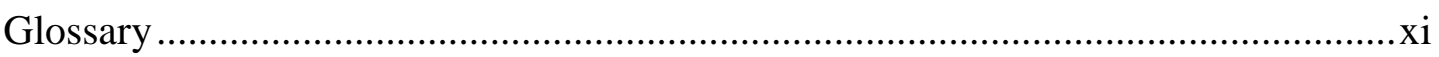

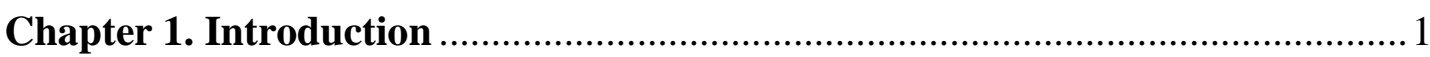

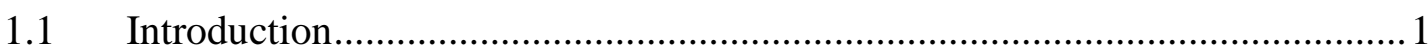

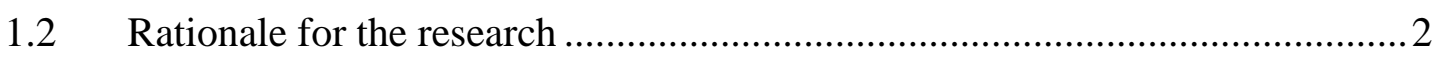

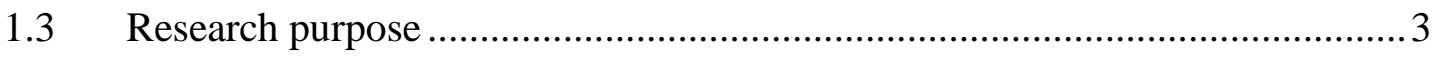

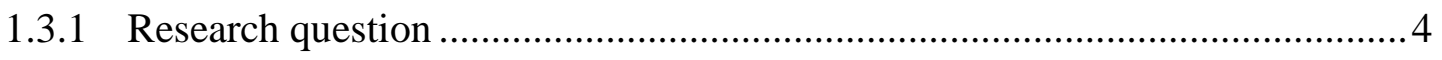

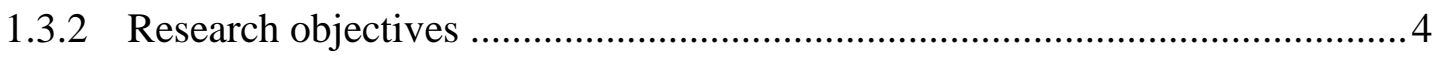

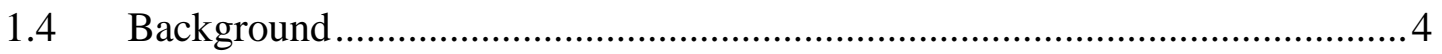

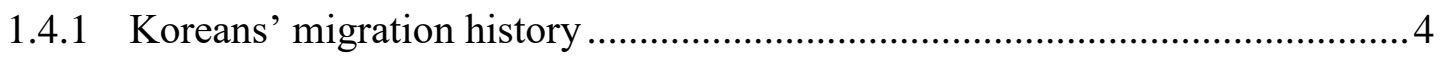

1.4.2 Korean immigrants in New Zealand ......................................................... 7

1.4.3 Healthcare system in Korea ................................................................... 11

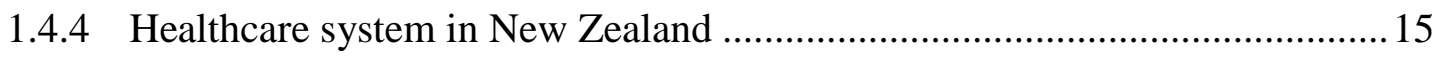

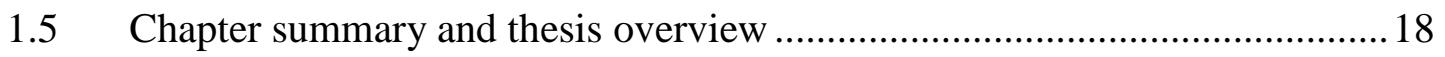

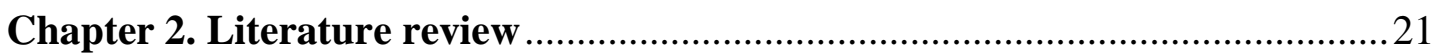

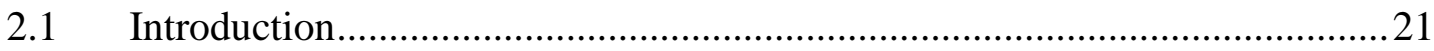

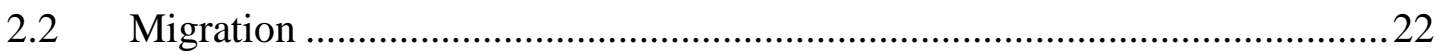

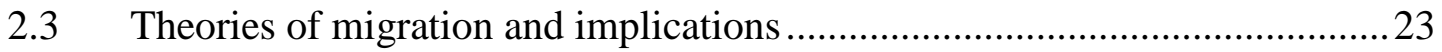

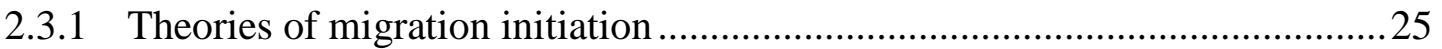

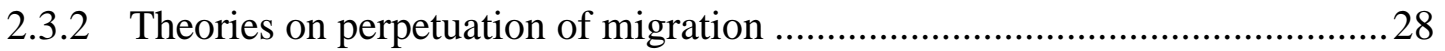

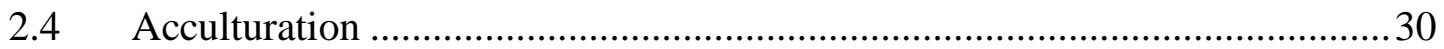

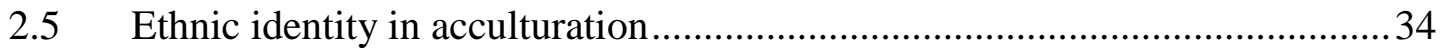

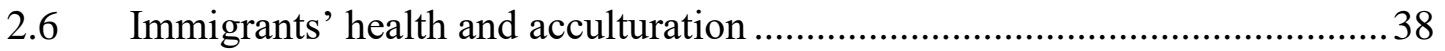

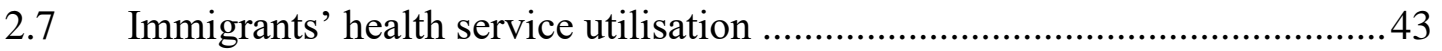

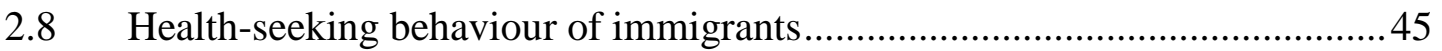

2.9 Korean immigrants' health-seeking behaviour in New Zealand ...................47

2.10 Illness perception and cultural implications .............................................49

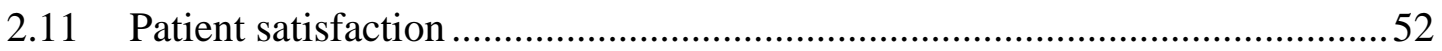




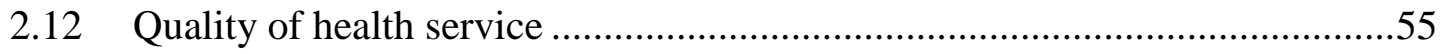

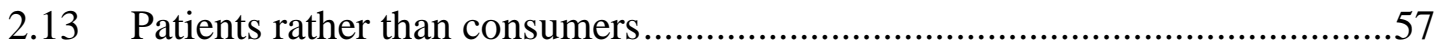

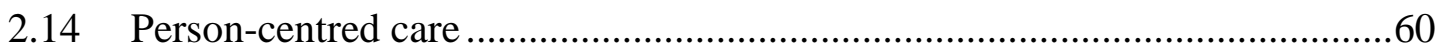

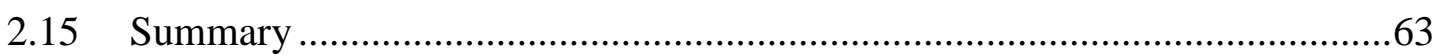

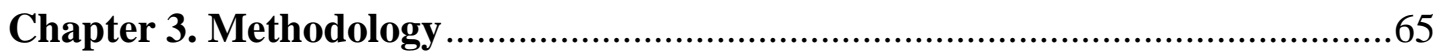

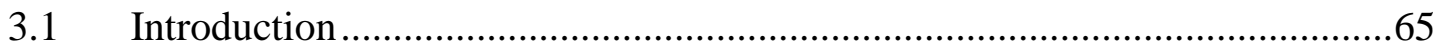

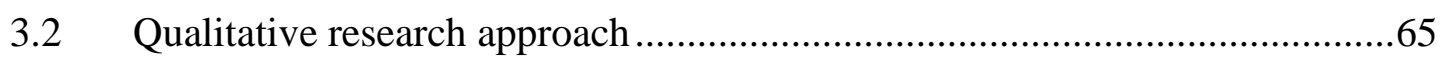

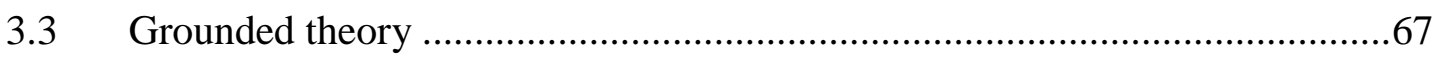

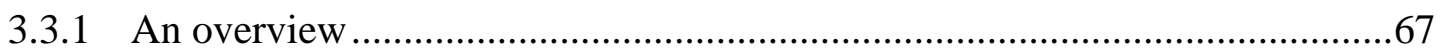

3.3.2 History and divergence of grounded theory..............................................68

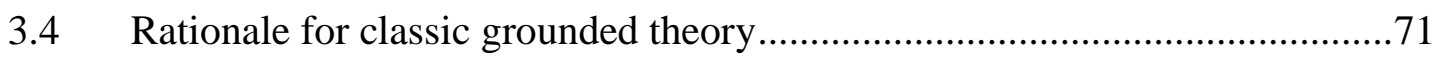

3.4.1 General methodology grounded theory....................................................

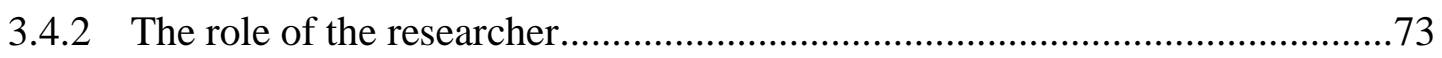

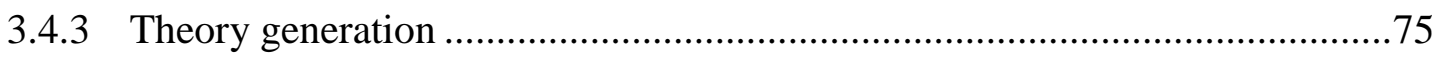

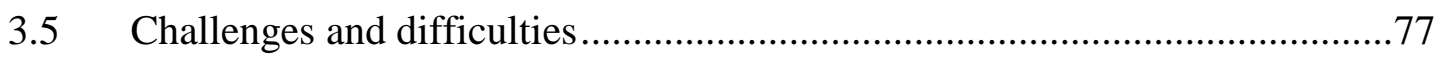

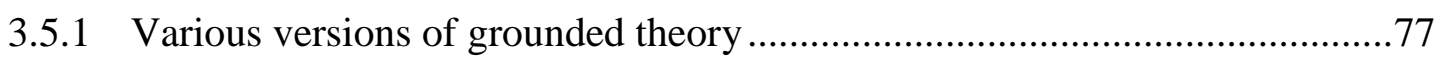

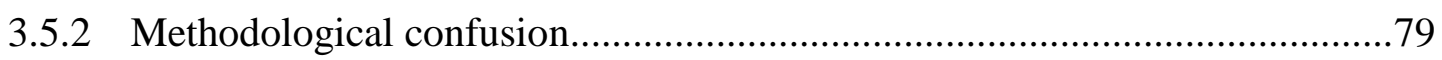

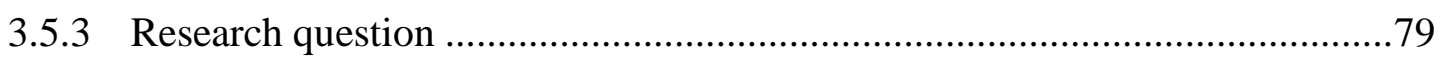

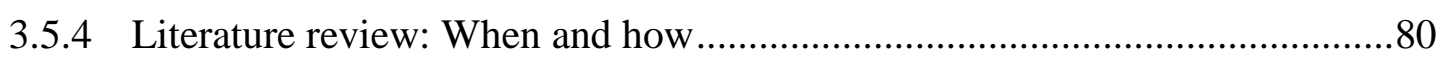

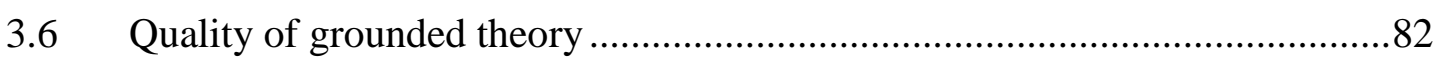

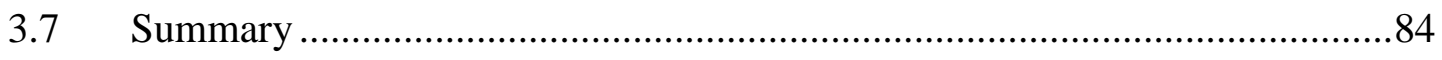

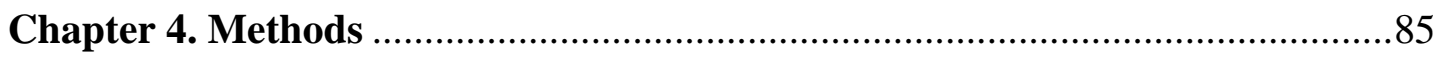

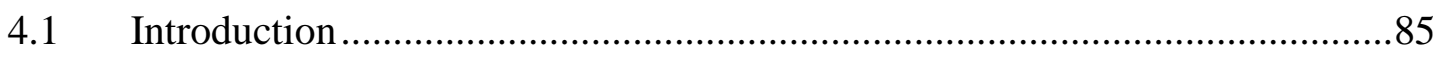

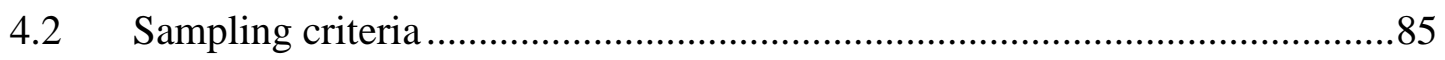

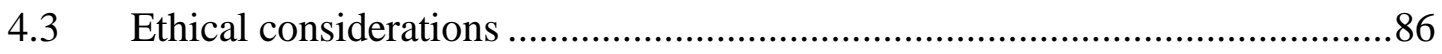

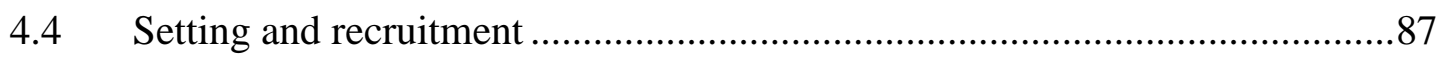

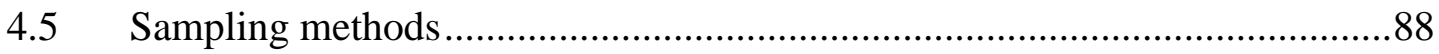

4.6 Characteristics of participants ............................................................... 90

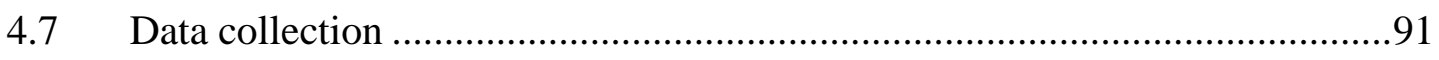

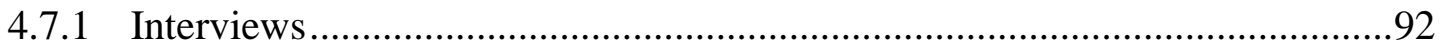

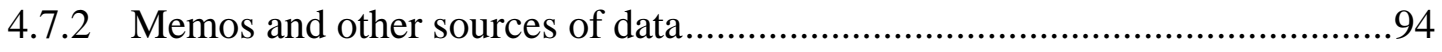

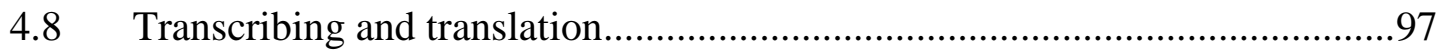

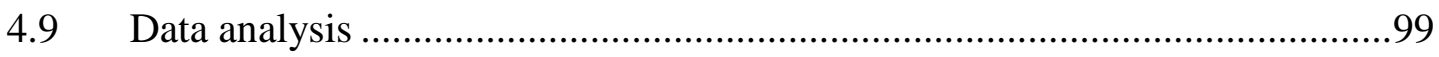

4.9.1 Substantive coding: Open coding and selective coding .............................100 
4.9.2 Constant comparison and theoretical sampling ........................................ 103

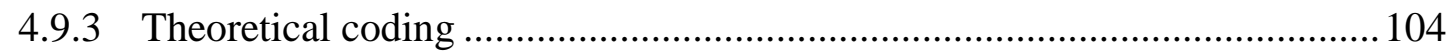

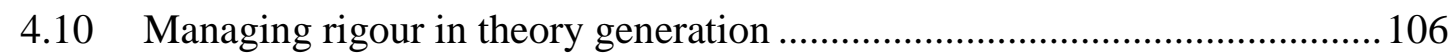

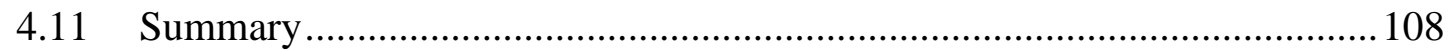

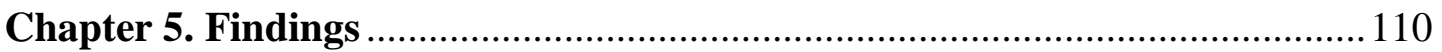

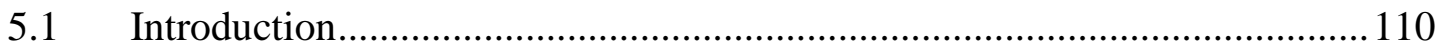

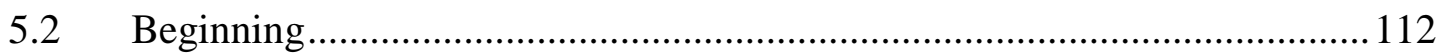

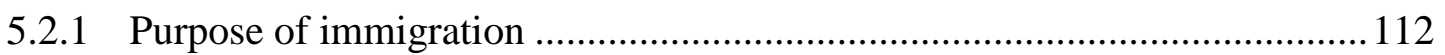

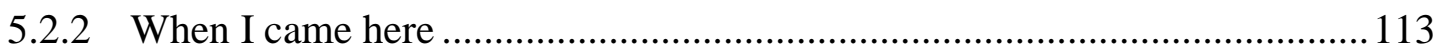

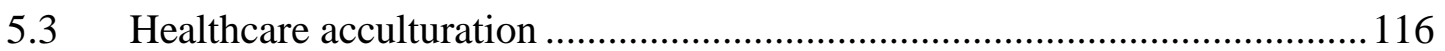

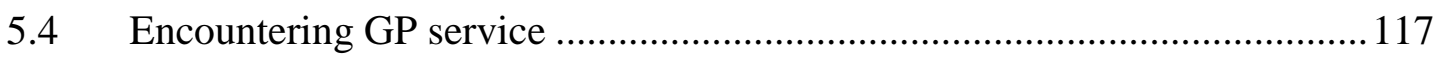

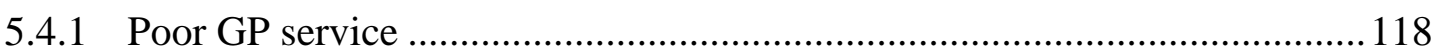

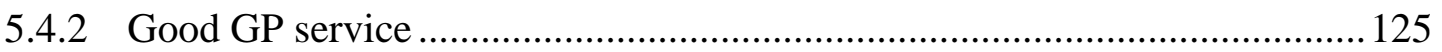

5.5 Four healthcare positions: Attuning, rejecting, attuning but negotiating,

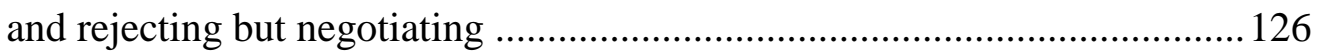

5.6 Rejecting: GP service and elective care at public and private services ........ 127

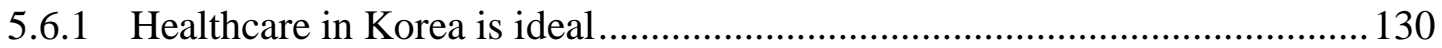

5.7 Attuning: Emergency care at public services ............................................. 139

5.7.1 Healthcare in New Zealand is ideal .......................................................... 142

5.8 Negotiating: Attuning but negotiating and rejecting but negotiating ............ 155

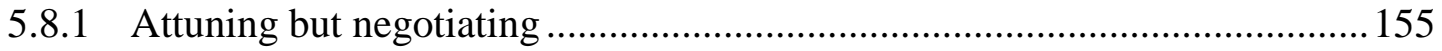

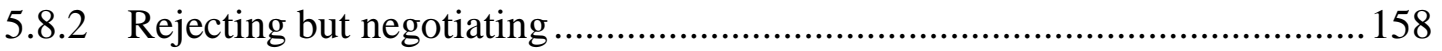

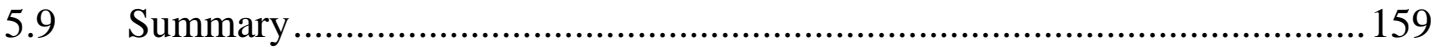

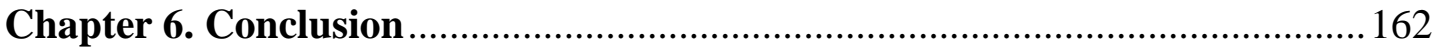

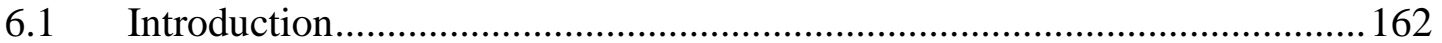

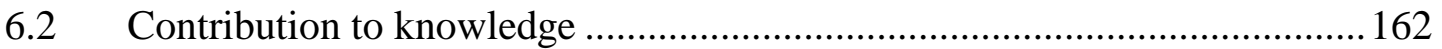

6.3 Contribution to methodology and methods ............................................... 166

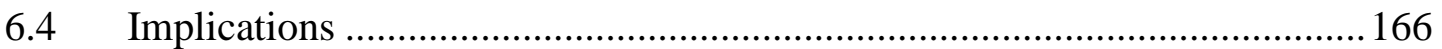

6.5 Limitations and recommendations for future research ................................ 168

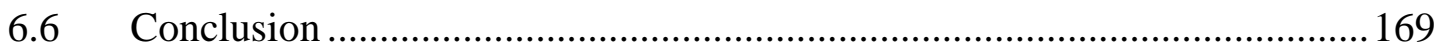

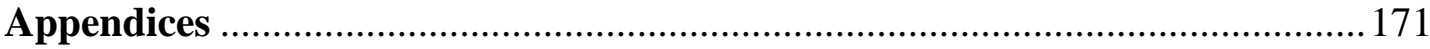

Appendix 1. Participant information sheet (Korean) ............................................ 171

Appendix 2. Participant information sheet (English) ........................................... 173

Appendix 3. Participant consent form (Korean) …............................................. 175 
Appendix 4. Participant consent form (English)...............................................176

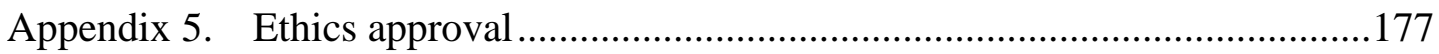

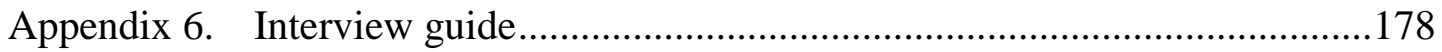

Appendix 7. Demographics of participants .........................................................179

Appendix 8. Data analysis and progress: Diagrams A, B, C ...............................184

Appendix 9. Data analysis and progress: Diagram D ..........................................185

Appendix 10. Data analysis and progress: Diagram E ...........................................186

Appendix 11. Development of codes .....................................................................187

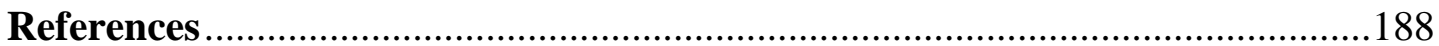




\section{List of figures}

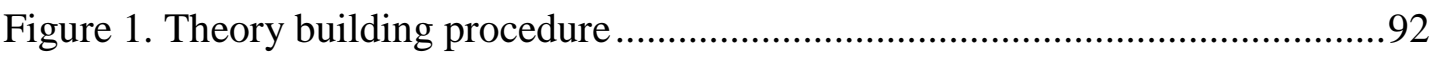

Figure 2. Healthcare Acculturation Theory …....................................................111

Figure 3. Healthcare Acculturation Theory: Encountering GP service ....................118

Figure 4. Healthcare Acculturation Theory: Rejecting ........................................127

Figure 5. Healthcare Acculturation Theory: Turning point and attuning .................140

Figure 6. Healthcare Acculturation Theory: Attuning but negotiating and rejecting

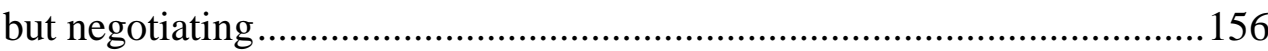

\section{List of tables}

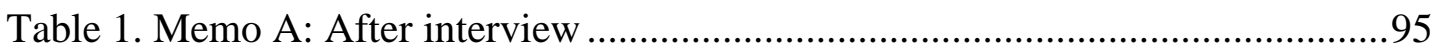

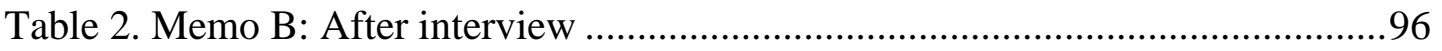

Table 3. Memo C: After informal conversation .........................................................97 


\section{Glossary}

Health-seeking behaviour is an outcome of sequential activities undertaken by individuals to manage health problems and protect their health from illnesses/diseases (Ward, Mertens, \& Thomas, 1997). This behaviour is influenced by individual's illness perceptions and health beliefs, and their healthcare activities, including remedial actions; health service utilisations are directed by this behaviour (Anwar, Green, \& Norris, 2012).

Acculturation is a multidimensional process of cultural and psychological changes that appear when individuals or groups of people are exposed to a new culture. It involves different degrees of culture learning and maintenance and related attitudes, values and behaviours (Organista, Marin, \& Chun, 2010).

Healthcare acculturation is an adapting process within the new host country's healthcare system which is a part of acculturation as a whole. While migrants interact with the host health services, their healthcare perceptions, attitudes and behaviours toward the host healthcare system are formed based on health services received in the host country and healthcare they experienced in their home countries. The process of healthcare acculturation is reflected in the migrants' healthcare perceptions, attitudes and behaviours; at the same time, the levels of healthcare acculturation influence migrants' lives as well.

Healthcare position reflects migrant's response towards the host healthcare system. Migrants choose one of these healthcare positions (rejecting, attuning, attuning but negotiating, and rejecting but negotiating) as their approach to healthcare based on their healthcare experiences and their perceptions of the host health services, and the chosen position affects their health service utilisation and health-seeking behaviours. However, migrants' healthcare positions are not end-outcomes in themselves as their healthcare experiences are ongoing. During the healthcare acculturation process their positions are enhanced, modified, or transitioned. 
Health service utilisation is the measure of the population's usage of the healthcare services available to them. This measure is used to examine how efficiently a health service system produces health in a population. The specific factors are the range and comprehensiveness of services, their accessibility, availability and affordability, and the continuity and co-ordination of care (Ministry of Health, 2010).

Healthy immigrant effect is the phenomenon where immigrants appear healthier than the native-born citizens due to health screening prior to immigration and self-selection of younger and more highly educated individuals among people considering migration (Kennedy, McDonald, \& Biddle, 2006; McDonald \& Kennedy, 2004; Mehta, 2012).

Medical return is a particular health-seeking behaviour whereby immigrants seek healthcare in their homeland (Horton \& Cole, 2011).

Migration is the temporary or permanent geographical movement of individuals or groups of people (Hagen-Zanker, 2008). The term covers both phenomena of people leaving (emigration) and coming into (immigration) a country and includes many different types of migrants; temporary and long-term labour (low and high skilled) and business migrants; undocumented or illegal migrants; refugees and asylum seekers; forced migrants caused by famine and natural disasters; migrants' family members (family reunion and formation); returning migrants (Organisation for Economic Co-operation and Development [OECD], 2006). 


\section{Chapter 1. Introduction}

\subsection{Introduction}

This research was initiated to render an understanding of the health-seeking behaviour of Korean immigrants in New Zealand which has received relatively little attention in the literature. As a minority subgroup in New Zealand, Koreans were found to be one of the fastest-growing ethnic groups in the 2006 census (Statistics New Zealand, 2006). They became one of the largest Asian ethnic groups, behind Chinese, Indians, and Filipinos (Statistics New Zealand, 2014b). However, there is a dearth of knowledge about Korean immigrants regarding their health and healthcare in New Zealand. This research sought to address the knowledge gap by developing a substantive theory grounded in Korean immigrants' healthcare experiences, perceptions, and behaviours to provide a theoretical explanation of their healthseeking behaviours in New Zealand.

This introductory chapter provides a general outline and background to the research. It begins with a rationale and justification for the research, followed by the purpose, question and objectives. Three relevant background areas pertaining to Korean immigrants and their health-seeking behaviours in New Zealand are introduced in this chapter: 1) Koreans' migration history; 2) the immigration purposes and settlement issues of Korean immigrants; and 3) the healthcare systems in Korea and in New Zealand. The overview of Korean migration historical background provides information on Koreans' immigration decisions and the initial reasons for immigration to New Zealand. The immigration expectations and settling issues of Korean immigrants in New Zealand are described and discussed in detail. The two different healthcare systems of Korea and New Zealand are outlined and compared to help readers understand Koreans' healthcare experiences and perceptions, as well as their health-seeking behaviours within the New Zealand healthcare system. 


\subsection{Rationale for the research}

When immigrants move to a foreign country they are expected to settle in a new environment and adjust to a new lifestyle; this means that immigration is not only a geographical relocation but also involves a social and cultural adaptation into the new society (Choi, 2013a). Although most Koreans immigrated to New Zealand looking for a better quality of life, they have been found to face settlement issues such as limited English proficiency, low income, employment problems and a lack of integration into a new society (Morris, Vokes, \& Chang, 2007; Song, 2013; Zhou, 2009). Furthermore, "medical return", which refers to going back to their homeland for medical care (Horton \& Cole, 2011), has been observed amongst Korean immigrants who have lived more than 10 years in New Zealand (Lee, Kearns, \& Friesen, 2010).

This phenomenon does not take into account healthcare during a holiday in their home country or medical tourism, which is medical treatment as part of a tour package (Turner, 2007). Medical return is "solely going back to one's home country for medical care or postponing treatment in order to have medical care when they next return to their home country". While a number of studies have confirmed a positive association between immigrant's health service utilisation and their levels of acculturation (Akresh, 2009; Fassaert, Hesselink, \& Verhoeff, 2009; Leclere, Jensen, \& Biddlecom, 1994; Salant \& Lauderdale, 2003), the first generation Koreans who have lived more than 10 years in New Zealand and who were eligible for free care, still go back to Korea for medical care despite putting their health at risk by delaying care. This health-seeking behaviour can be seen as a failure to negotiate or adapt into the healthcare system in New Zealand (Elliott \& Gillie, 1998; Ormond, 2014). How Korean immigrants perceived healthcare in New Zealand and how they responded to it; and what factors affect their healthcare decisions and health-seeking behaviours are ambiguous. The main concerns of Korean immigrants regarding their healthcare and how they resolve them are not well known to New Zealand society and even to Koreans themselves, since there is little research pertaining to Koreans' health and health-seeking behaviour. Any reference to their health is embedded in discussion 
about the health of Asian or other Asians in New Zealand because Koreans have been categorised as "other Asians" (Mehta, 2012).

I am a Korean who lives in New Zealand and share the same healthcare background as the participants in this research, which is very different to that of New Zealand. Being one of them, I could see the differences in healthcare between Korea and New Zealand and I could understand the difficulties in utilising the New Zealand health services due to the existing gap between the two systems. However, being new here, I did not know what other Korean immigrants' main concerns related to their health and healthcare were and how they resolved them; as these were not found in the literature. As a researcher who majored in public health, I was interested in Koreans' health-seeking behaviours in New Zealand. I wanted to know how Koreans perceived healthcare in New Zealand; what factors contributed to their healthcare perceptions and health-seeking behaviours; and how their health-seeking behaviours were reconstructed and maintained or transitioned during their healthcare acculturation processes.

To understand the main concerns of Korean immigrants in healthcare, this research explored their healthcare experiences in New Zealand and in Korea including their perceptions of these experiences and expectations; and examined the process of healthcare acculturation of Korean immigrants within the New Zealand healthcare system. Korean immigrants' healthcare experiences were theorised, and the developed theory will provide insights into their healthcare adapting processes as well as those of other ethnic immigrant groups, since there is no theory that explains immigrant's healthcare adapting processes. Consequently, the findings of this thesis will help in addressing and filling the gap in knowledge of Korean immigrants' health-seeking behaviours in New Zealand.

\subsection{Research purpose}

This research was designed to develop a substantive theory to render insights into Korean immigrants' health-seeking behaviours in New Zealand, and to address how Korean immigrants interact with the New Zealand healthcare system during their healthcare acculturation process. 


\subsubsection{Research question}

What are the health-seeking behaviours of Korean immigrants in New Zealand and what factors affect these behaviours? ${ }^{1}$

\subsubsection{Research objectives}

To achieve the purpose of the research, the research objectives were:

1) to explore the health-seeking behaviours of Korean immigrants in New Zealand;

2) to describe healthcare perceptions of Korean immigrants and their transition pathways in the healthcare acculturation process;

3) to identify the factors that influence Korean immigrants' health-seeking behaviours;

4) to provide a theoretical explanation for these experiences.

\subsection{Background}

\subsubsection{Koreans' migration history}

The Korean diaspora is almost 7.2 million worldwide (Ministry of Foreign Affairs, 2015) which is equivalent to approximately $14 \%$ of the South Korean population (51 million in 2015) (Statistics Korea, 2015). Furthermore, South Korea (henceforth Korea), previously the world's most ethnically homogeneous nation ${ }^{2}$ has recently adopted multiculturalism as official policy in order to handle a rapidly growing population of foreigners (Prey, 2011). The Korean international migration history started in the 1860s (Joseon Dynasty, 1392-1897) when many Korean peasants moved to China and Russia to escape from hunger (Oh et al., 2011). Koreans who migrated to Hawaii (1903) as labourers in the sugarcane plantations were the first official English language passport holders issued by the Empire of

\footnotetext{
${ }^{1}$ This research question was developed at the beginning of the research to complete the research proposal. It implied broad and general interests of the research in order to stay open and flexible to the data.

2 The Korean ethnic group accounted for $98.1 \%$ of the total population in Korea in 2006 and it has decreased to around $96.4 \%$ in 2014 (Statistics Korea, 2015).
} 
Korea (1897-1910) (Baik \& Murabayashi, 2011). During the Japanese colonial regime from 1910 to 1945, Korean migrants included asylum seekers who continued to move overseas to have a better life (Oh et al., 2011). Korea had been one of the world's poorest countries until the Korean War (1950-1953). After the Korean government was established (1948), migration trends in Korea were in three categories in chronological order; the age of labour sending (emigration) from the 1960s to the 1990s; the age of labour receiving (immigration) from 1990 to 2003, and the age of increasing foreign residents from 2004 to date (Oh et al., 2011).

\section{The age of labour sending (1960s - 1990s)}

In the early 1960s, emigration was encouraged by the Korean government as a strategy to control population, alleviate unemployment, generate inflow remittance, and acquire advanced technology (Kwon, 1997). Around 21,000 contract mine workers and nurses were dispatched to Germany (1963-1978), and people began emigrating to the United States of America (USA) to study or for better income after the USA opened its doors to Asian immigrants in 1965 (Kwon, 1997; Oh et al., 2011; Park, 2004). In the 1970s and 1980s, millions of Korean labourers emigrated to the oil-rich countries of the Middle East as contract workers, and they returned home with substantial savings after a few years (Park, 2004). The number of labourers who emigrated rose dramatically from 14,500 in 1974 to 434,000 in 1983, and Korea was a labour sending country until the early 1990s (DeWind, Kim, Skeldon, \& Yoon, 2012). At that time, the remittances from Korean emigrant labourers grew not only their family economy but also contributed to the Korean economy (Kwon, 1997). During this period, emigration in Korea mainly occurred because of the differences in wage and employment conditions. This migration pattern can be explained by Neo-classical Migration Theory ${ }^{3}$ which considers migration as an individual's choice and a part of the process of economic development (Harris \& Todaro, 1970; Massey et al., 1993).

\footnotetext{
${ }^{3}$ See the Section 2.3.1 Theories of migration initiation (p. 25).
} 


\section{The age of labour receiving (1990 - 2003)}

The growing demand for workers resulted from economic development and political settlement in Korea. Prior to 1990 there were more than 430,000 Korean labour emigrants, and this number dropped greatly to 18,000 by 1993 (DeWind et al., 2012; Kim, 2009a; Oh et al., 2011). Simultaneously, a new phenomenon, the immigration of foreigners, especially foreign labourers into Korea, started and increased (Oh et al., 2011). In 1993, the Korean government introduced the "Industrial Trainee System" and officially invited foreign trainees from 15 Asian countries to become a special part of the Korean workforce (Oh et al., 2011). Massey (2003), an American sociologist explained that economic growth in Asian countries (Taiwan, Korea, Hong Kong, Singapore, Thailand, and Malaysia) had brought a transformation in their migratory pattern from sending (emigration) to receiving (immigration) labour.

Meanwhile, at this time, marriage immigration was introduced in Korea to facilitate the marriage of single rural men to foreign women from China, Indonesia, Mongolia and Southeast Asia (DeWind et al., 2012; Oh et al., 2011). Between 1990 and 2005, 160,000 Korean men married foreign women, and 80,000 Korean women married foreign men (DeWind et al., 2012).

\section{The age of increasing foreign residents (2004 - present)}

Korea has experienced great transitions in recent decades, such as industrialisation, urbanisation, and demographic transition with low fertility and mortality (DeWind et al., 2012). As a consequence of the economic growth, educated and affluent Koreans began to avoid certain occupational categories, particularly the so-called “3-D” (difficult, dirty, and dangerous) manual jobs (Kim, 2009a; Skrentny, Chan, Fox, \& Kim, 2007). Subsequently, Korea supported the entry of significant numbers of migrant labourers; with the number of migrant workers arriving in Korea growing exponentially from approximately 18,000 in 1990 to nearly 550,000 in 2008 (DeWind et al., 2012; Kim, 2009a). According to the Dual Labour Market Theory ${ }^{4}$, the Korean labour market at that time can be divided into two sectors: the primary

\footnotetext{
${ }^{4}$ See the Section 2.3.1 Theories of migration initiation (p. 25).
} 
with attractive jobs, and the secondary with 3-D jobs (Jennissen, 2007; Kim, 2009a; Skrentny et al., 2007). Koreans take the attractive jobs, while the secondary jobs are taken by foreign workers. It can be noticed that the previous push factors of migration in Korea (low wages, high unemployment rates, and inferior working and living conditions) switched to pull factors (relatively wealthier economic situations and better working and living conditions); and these pull factors attracted foreigners especially foreign labourers to Korea, as well as Koreans from other countries.

By the middle of 2015, the foreign population in Korea totalled more than 1.7 million, an increase from 92,000 in 2009 , and accounted for $3.3 \%$ of the total population (Ministry of the Interior, 2015). Meanwhile, the number of Koreans who immigrated to foreign countries dropped from 26,236 in 2006 to 7,367 in 2014. This decline has also been found amongst Koreans who moved to New Zealand; the number fell from 1,574 in 2006 to 96 in 2014 (Ministry of Foreign Affairs, 2015). The Korean population in New Zealand dropped slightly from 30,792 (2006) to 30,171 (2013) within this period (Statistics New Zealand, 2006, 2014b).

\subsubsection{Korean immigrants in New Zealand}

Bicultural New Zealand, with mostly Māori (indigenous) and European/Pākehā $\bar{a}^{-5}$ (non-Maori settlers) until around the 1960s, has increasingly become a more culturally and ethnically diverse society (Thorns, Fairbairn-Dunlop, \& Du Plessis, 2010). New Zealand is known internationally as one of the highest immigrant receiving countries in the world (Singham, 2006). According to the 2013 Census, more ethnicities (213) reside in contemporary New Zealand than there are countries in the world (196), and this great diversity of New Zealand is growing (Statistics New Zealand, 2013). This diverse society is often described as one nation, two peoples and many cultures (Bozic-Vrbancic, 2003). However, biculturalism is still an official policy in New Zealand based on the Treaty of Waitangi ${ }^{6}$, and

\footnotetext{
${ }^{5}$ Pākehā, which is a Māori term for white man, refers to non-Maori or to New Zealanders who are European (Ballara, 1986).

${ }^{6}$ The Treaty of Waitangi (also known as "Te Tiriti o Waitangi” in Māori) is an agreement between the British Crown and the Maori in 1840; as equal partners with equal rights and privileges (Smith, 2010).
} 
multiculturalism has not been declared as an official policy (Bozic-Vrbancic, 2003; Smith, 2010). New Zealand's population is comprised of diverse ethnic groups: European (74.0\%), Māori (14.9\%), Asian (11.8\%), Pacific peoples (7.4\%), Middle Eastern/Latin American/African (1.2\%), and many other minority ethnic groups.

The majority of Korean immigrants arrived in New Zealand in the 1990s and the most significant growth in the Korean population in New Zealand was reported at that time; from less than 500 in 1986 to nearly 13,000 in 1996 (Statistics New Zealand, 2007). Since Koreans' immigration history in New Zealand is relatively short compared to Chinese and Indians, most (89\%) Koreans in New Zealand are first-generation who were born overseas (Statistics New Zealand, 2014a). They are generally concentrated in the main urban areas (96.2\%), such as the Auckland Region where more than two-thirds of the Korean population reside (72.9\%), followed by the Canterbury Region (11.1\%), and the Waikato Region (3.7\%). Their ethnicity was predominantly Korean $(97.2 \%)$ and the most common language spoken was Korean $(85.9 \%)$.

\subsubsection{Purpose of immigration}

Koreans, who were wealthy, young, and educated, immigrated to New Zealand under the "points system" (Lee, Kearns, et al., 2010). Yoon and Yim's (1997) study on the occupations of Korean immigrants in Auckland explained that most Koreans immigrated to New Zealand looking for a better quality of life rather than for economic reasons. Koreans chose the quality of life in New Zealand over an economically comfortable life in Korea. This meant, when middle or upper middle class Koreans made decisions to immigrate to New Zealand, they probably knew that their future income would not be greater than their income in Korea. Rather they were attracted by pull factors in New Zealand such as a more relaxed and better lifestyle, clean environment, and an English-speaking environment for their children's education (Kim \& Yoon, 2003; Lidguard \& Yoon, 1998; Morris et al., 2007). They were also pushed from Korea due to the competitive education system, stressful working conditions, and crowded and polluted living environment. 
Lee (2012), a Korean professor of economics, analysed the main factors of happiness in the Organisation for Economic Co-operation and Development (OECD) countries. He measured weights and rankings of these countries based on the OECD Better Life Index ${ }^{7}$. He found that Koreans were the second unhappiest people (after Hungary) amongst the OECD countries. The most relevant factors affecting Koreans' happiness were stable income and employment, and living conditions; and fairness in income distribution. Moreover, Korean teenagers might be the unhappiest in the world according to the Comprehensive Survey of Children in Korea (2013) (Kim, 2015). The survey, measuring children's subjective well-being against that of 30 OECD countries, found that $60.3 \%$ of Korean children had high life satisfaction compared to $94.2 \%$ of children in the Netherlands and $90.2 \%$ of children in Iceland. Korean children's life satisfaction ranked at the bottom of the OECD countries. The primary reason for their low level of life satisfaction was the pressure to achieve high grades at school--a requirement to enter top-class universities--and to achieve this they had to take private lessons after a full-day school programme (Park, 2014, Nov 04). In Korea, the university entry criteria include the admission examination scores and the student's grades over all three years of high school. Typically, students in the 3rd grade leave home early in the morning with three meal boxes (breakfast, lunch and supper) and come home very late at night (around midnight). In this educational system, children have to work long hours to compete with others. Most Koreans believe that having a good educational background is one of the best ways to improve social status, and over-emphasis in this belief has become a burden to both students and parents (Jones, 2013; Schneidewind, 2016).

\subsubsection{Settlement}

Most Koreans moved to New Zealand looking for a better quality of life; however, it seems that they confront settlement issues in New Zealand (Morris et al., 2007; Song, 2013; Zhou, 2009). According to a study on the healthcare needs assessment for Asian people in Waitemata (Zhou, 2009), Koreans were the least able

\footnotetext{
${ }^{7}$ The OECD Better Life Index is a tool which provides 34 OECD countries' performance of wellbeing across 11 dimensions, such as health, education, environment, overall satisfaction with life, and income (OECD, 2011).
} 
to speak English amongst Asian ethnic groups; nearly 30\% of Koreans were with "No English" while only 5\% of Indians were not able to speak English. The data in Zhou's study came from national surveys and statistical reports in New Zealand. In addition, a survey of 479 Korean immigrants in Auckland in 2006 (Han et al., 2007) found that $78 \%$ of respondents spoke only Korean at home and $58 \%$ claimed the language problem as the most difficult factor for settlement.

The 2013 New Zealand Census showed that the median income for Koreans who are aged 15 or over was NZ\$11,500 while it was NZ\$27,400 for Indians and NZ\$37,300 for New Zealanders (Statistics New Zealand, 2014c). Almost half of the working-age (15+) Koreans (44.5\%) were not in the labour force while that of Indians and New Zealanders were $28.0 \%$ and $32.9 \%$ respectively. Only $34.9 \%$ of the working-age Koreans had full time jobs, but $53.5 \%$ of Indians and $48.0 \%$ of Europeans had full time jobs (Statistics New Zealand, 2014c). Chang, Morris, and Vokes (2006) found that Koreans' underemployment (employment in unskilled and low paid jobs according to their qualifications and careers) remained a problem as well. As a result of the serious inability to gain employment, many Koreans established small businesses such as grocery stores, restaurants, souvenir shops, cafes and travel agencies (Song, 2013; Yoon \& Yim, 1997). The number of Korean businesses has gradually grown in line with the increase in the number of Korean residents; the 37 listed Korean businesses in Auckland grew to approximately 636 between 1992 and 1997, and to around 1,000 in 2005 (Han et al., 2007). Of note, most of these businesses rely heavily on Korean customers, suppliers, and labourers; and can be referred to as typical co-ethnic businesses (Cain \& Spoonley, 2013; Song, 2013; Yoon \& Yim, 1997).

Research by Morris et al. (2007) focusing on the experience of "social exclusion" of Korean immigrants in Christchurch claims that fitting into the host society is one of the biggest challenges for Korean immigrants in Christchurch. Most of the 36 participants in this research experienced incidents of harassment and complained of the difficulties in making Kiwi friends. Similarly, according to the pilot longitudinal survey (2001-2002) on migrants' settlement experiences, more than half of the migrants including Korean immigrants did not have any New 
Zealander friends (New Zealand Immigration Service, 2004). The total survey population was 691 at Wave 1 (2001) and 546 at Wave 2 (2002). Koo (2004) who examined the adaptation process and mental health of Korean immigrants in Christchurch (based on his ethnographic Master's thesis completed in 1997) criticised the Koreans' attitudes. In his view, Koreans experience social exclusion due to their lack of desire to fit into the new society. In contrast, Morris and her colleagues (2007) argue that Koreans who immigrated to New Zealand expected to become a part of New Zealand society and did not want to mix only with Koreans. However, they gave up this desire when they experienced social exclusion and rejection such as racism, discrimination, and harassment (e.g., rejection from personal or private meetings, verbal abuse, and actual physical attacks).

\subsubsection{Healthcare system in Korea}

Korea has a universal healthcare system, provided by a compulsory National Health Insurance (NHI) and operated by a non-profit organisation, National Health Insurance Corporation (NHIC) under government supervision (Ministry of Health, Welfare, and Family Affairs of the Korean Government) (Chun, Kim, Lee, \& Lee, 2009; Kim, 2010). Nearly the entire population (approximately 97\%) is covered by the NHI, and the remainder (approximately 3\%) who are unable to pay for their own healthcare coverage are supported by the Medicaid-Aid program (MAP) (Kwon, Lee, \& Kim, 2015; Lee \& Shaw, 2014). The insured in NHI are divided into "employee" and "self-employed" groups. The employee insured group includes the insured persons, their spouse, direct lineal descendants and ascendants, and unmarried brother and sisters. The self-employed insured includes persons excluded from the employee group (National Health Insurance Service, 2014). A uniform contribution rate is required from all employees (5.99\% in 2014); employees pay $50 \%$ of the contribution and their employers pay the other $50 \%$. The contribution of selfemployed insured is based on both income and household assets (e.g., property, house, and vehicle) (National Health Insurance Service, 2014).

The Korean healthcare system is financed almost equally by public and private funding; public 55\% and private $45 \%$ in 2011 (Kwon et al., 2015). The public 
sector consists of individual member contributions and government subsidy. While the individual contributions accounted for $82.7 \%$ in 2013, the government subsidy was $17.3 \%$ (National Health Insurance Service, 2014). The private sector is mostly funded by out-of-pocket payment for non-covered and co-payment on covered services which accounted for $35.2 \%$ in 2011 (Kwon et al., 2015). The proportions of the patients' co-payment are $20 \%$ for the insured services in inpatient care and a differing proportion of cost sharing (30-60\%) depending on the level of health services for outpatient care (Kwon et al., 2015; National Health Insurance Service, 2014).

The three major characteristics of the Korean healthcare system are: a private sector dominant healthcare provider system, patient's freedom to choose provider, and a functional undifferentiated healthcare delivery system (Ock et al., 2014; OECD, 2002). Healthcare delivery in Korea is largely privatised and the private healthcare providers play a major role in the Korean healthcare system; about $94 \%$ of hospitals and nearly all clinics were private providers in 2011 (Kwon et al., 2015). Although the rate of the patients' co-payments (out-of-pocket payments) has declined from 50\% in 1995 to $35 \%$ in 2011 , it is still relatively high compared with the OECD average of $20 \%$ (2011) (OECD, 2013). This substantially higher rate of out-of-pocket payment in Korea is mainly incurred by the consumption of the uninsured services which mostly involve new high cost medical technologies and medicines offered by healthcare providers for profit reasons (Kwon et al., 2015). Healthcare providers are paid based on the fee-for-service (FFS) payment method in Korea; the expenses are calculated and the unit price per score is determined by negotiation between the NHIC and healthcare providers (National Health Insurance Service, 2014). Under the FFS payment system, healthcare providers, particularly private providers tend to encourage induced demand, and Korea is no exception (Goldsmith, 2012; Lee \& Shaw, 2014; OECD, 2012). In the last 15 years, Koreans' healthcare expenditure has doubled; the total healthcare expenditure was $3.7 \%$ of GDP (Gross Domestic Product) in 1995 and has increased to 7.4\% in 2011 (Kwon et al., 2015). It was one of the highest growth rates amongst OECD countries even though they were still low compared to the OECD average for healthcare expenditure of $9.3 \%$ in 2011 (OECD, 2013). 
In Korea, medical facilities are divided into three categories depending on the number of medical departments and the size of the facilities (Ministry of Health and Welfare, 2012). The first-tier facilities are clinics with less than 30 beds, including clinics for maternity and dental care, and oriental medicine. They provide medical services for early symptoms of diseases. Secondary facilities are hospitals, large scale general hospitals, dental hospitals, and Korean traditional medical hospitals (30 to 300 beds); and they offer specialized high quality care for inpatients and outpatients. Tertiary medical care focuses on high complexity medical services and is provided by general hospitals or hospitals belonging to medical schools. There are 44 tertiary hospitals designated by the government (National Health Insurance Service, 2014). Since there is no gatekeeping role in the Korean healthcare system, patients do not need to register with healthcare provider(s), and they are not required to have a referral to see a specialist (Chun et al., 2009; Kwon et al., 2015; Song, 2009). Patients freely choose their own doctors and hospitals according to their preference irrespective of the levels of health. Referral is required for the tertiary care in Korea; however, accessing tertiary hospitals is relatively easy because the Korean government does not strictly enforce its access (Chun et al., 2009; Kwon et al., 2015). Patients have to pay $60 \%$ of the total medical service cost and doctor's fee without referrals; nevertheless, the main healthcare providers in Korea are secondary and tertiary institutions (World Health Organization \& Ministry of Health and Welfare, 2012).

Without a gatekeeping role in primary healthcare, hospitals and clinics in Korea provide similar services because their roles and functions are not clear (Kwon et al., 2015; Lee, Park, Choi, \& Kwon, 2010; Ock et al., 2014). Accordingly, there is serious competition between them within the market-oriented healthcare delivery system; they compete with each other like normal businesses to attract consumers (patients) in order to achieve higher profits (Kwon et al., 2015; Ock et al., 2014; OECD, 2012). While the unit fees per healthcare service in Korea were lower compared to other OECD countries as a consequence of this competition, there was also an increased usage of healthcare services that lie outside the health insurance benefit which is derived by the healthcare providers for their profits (OECD, 2012). Hospitals in Korea are allowed to respond to the patients' healthcare demands 
directly and patients literally shop for their doctors (Kwon et al., 2015; Lee, Choi, et al., 2013). In this circumstance, providers are likely drive healthcare demand by having as many patients as possible and providing unnecessary healthcare including non-insured medical services to maximise their profits (Goldsmith, 2012; Lee \& Shaw, 2014; OECD, 2012). Consequently, there is an oversupply of health services and excessive care in Korea.

Large scale private hospitals in Korea offer various healthcare services with advanced medical technologies and specialised doctors (Lee, Park, et al., 2010). Patients in Korea are used to walking in without an appointment and self-referring to specialists. The norm is of a one-stop-shop system of healthcare where various health services are available at one place (Noh et al., 2006; Tse, Wong, Hong, \& Rasalingam, 2013). For example, patients are able to access many different health services, such as consultation, medical treatments, operations, blood tests, radiology tests, and prescriptions in a hospital on the same day. Koreans typically have extensive investigations and access to a battery of check-ups. People aged 40 or over are entitled to take regular "General Health Check-ups" (also known as comprehensive body checks) every two years (every year for the employee insured excluding office workers), which is covered by the NHI (National Health Insurance Service, 2014, p. 21). There is also a demand for private comprehensive medical checks for more sophisticated tests, and private hospitals offer various medical check-up programmes to meet the demand. The cost of medical check-ups varies depending on the types and the number of screenings and on the hospital. Each hospital has its own cost structure (the minimum price for a check-up programme is approximately NZ\$700-800) (Asan Medical Center, 2016; Kim, Kim, Park, Park, \& Cho, 2015; "Medical screening," 2014). Koreans were able to self-purchase most medications from local pharmacies until 2000 when a new Korean government policy prohibited physicians from dispensing and pharmacists from prescribing drugs by law (Kim \& Ruger, 2008; Park et al., 2005). What is now required is a physician's prescription which a pharmacist fills.

Koreans living overseas with a F-4 visa (people of Korean heritage) are eligible for the NHI Program after residing back in Korea for at least three months 
(Immigration Bureau, 2005; Song, 2009). According to the Ministry of Health and Welfare (MOHW) (2011), the number of overseas Koreans who travel to Korea for medical care and examinations increased from 10,789 people in 2003 to 25,009 people in 2011.

\subsubsection{Healthcare system in New Zealand}

New Zealand has a universal coverage healthcare system, provided by public, private and non-governmental sectors (Cumming et al., 2014). Healthcare in New Zealand is predominately financed by public funding through general taxation (taxbased financing). Between 2009 and 2010, 83.2\% of healthcare expenditure was funded publicly including $8.4 \%$ of contribution from the Accident Compensation Corporation (ACC) and 2.0\% from other government resources (Ministry of Health, 2012a). The other $16.8 \%$ of total healthcare expenditure was covered by private funding: $10.5 \%$ of households' out-of-pocket payments; $4.9 \%$ of private insurance; and $1.4 \%$ of non-government funding. This proportion of patient out-of-pocket payments $(10.5 \%)$ was lower than that of the OECD average $(20 \%)$, and the total healthcare expenditure of GDP $(10.3 \%)$ was above the OECD average $(9.3 \%)$ in 2011 (OECD, 2013).

While the New Zealand healthcare system is largely funded through public sources, the delivery system is a mix of public and private services (Medical Council of New Zealand). The Ministry of Health (MOH) is the government's principal advisor on health policy and disability support services; 20 District Health Boards (DHBs) are responsible for planning and funding health services for their geographical areas (Ministry of Health, 2015b). Twelve district health board-owned Public Health Units (PHUs) provide regional public health services focusing on improving, promoting, and protecting community health; and delivering efficient and effective healthcare to meet the health needs of people (Cumming et al., 2014). New Zealand's primary health care (PHC) is coordinated through 32 community-based primary health organisations (PHOs) ${ }^{8}$ (Cumming et al., 2014; Quin, 2009).

\footnotetext{
${ }^{8}$ PHOs, which were established in 2002, are not-for-profit organisations (Cumming et al., 2014).
} 
While public health services are funded by government, general practitioners (GPs) who provide PHC service are predominantly private doctors (Gauld et al., 2012). GPs, nurses and other health professionals (such as health promotion workers, dieticians, pharmacists, psychologists, and midwives) at a PHO provide a set of treatment and preventive services to their enrolled patients, and the government subsidises the PHO on a per capita basis (Cumming et al., 2014). There are also patient co-payments for GP services; patients pay additional fees according to their service usage (which is partial fee-for-service). The fees for GP services vary for each PHO because they are set by GPs themselves. GPs, who have practiced independently, have been encouraged to join or form PHOs together with other GPs and healthcare practitioners in order to achieve a population approach to primary care with the government funding (Neuwelt \& Matheson, 2012). Finally, most GPs are now members of PHOs (Ministry of Health, 2016). New Zealanders are also expected to be enrolled at a PHO for the GP service; and patients who are not registered with a PHO can still access the GP service but their fees are higher than those who are registered (Cumming et al., 2014). Between 2013 and 2014, 95\% of New Zealanders were registered with a PHO (Ministry of Health, 2014a).

Patients have access to the GP service by booking, but to access specialist care they need a referral letter (Cumming et al., 2014). PHC is the most important gateway to the public healthcare system; GPs act as gate-keepers of the healthcare system and control access to secondary and tertiary public services in order to provide timely healthcare when necessary (Jatrana \& Crampton, 2009). That is, except for accident and emergency care, patients cannot see a specialist without a GP's (or midwife's) referral, not only for specialist care at the secondary and tertiary public hospitals but also for private sector healthcare (Cumming et al., 2014; WHO \& Ministry of Health, 2012). Referrals from GPs, nurse practitioners, and specialists are required for all diagnostic tests such as laboratory and radiology tests. The 2008 Commonwealth Fund International Health Policy Survey found that New Zealand patients generally had easy access to their doctors (GPs), but their waiting times to see specialists were longest amongst other countries (Australia, France, Germany, Netherlands, UK, and USA) (Schoen, Osborn, How, Doty, \& Peugh, 2009). The New Zealand Health Survey 2013/14 showed that difficulties in making an appointment 
with their GPs, within 24 hours, and the high cost of the GP service was the main reasons for patients' unmet healthcare needs (Ministry of Health, 2014a). There were no unmet needs reported with respect to referrals to access specialist care or diagnostic tests because this was excluded in the indicators of the survey. The Accident Compensation Corporation commonly referred to as ACC is a government funded no-fault personal injury scheme for accident-related care covering New Zealanders and visitors to New Zealand (Ministry of Health, 2015a). ACC is a social insurance scheme paid for by a levy by all employers, employees and self-employed people, and funds accident and work related injury care at public hospitals and private healthcare sectors (Cumming et al., 2014).

Since 1958, the cost of the secondary and tertiary healthcare and disability support services has been paid by the government: inpatient or outpatient treatment in public hospitals; maternity care; mental healthcare; palliative care; dental care for school children (under 18) including prescription drugs, X-rays, and laboratory services (Cumming et al., 2014; Lang, 1987; WHO \& Ministry of Health, 2012). Patients pay fee-for-service co-payments for the GP service (children under six generally pay no fees), and for pharmaceuticals, elective surgeries, private hospital or specialist services and adult dental care. Complementary and alternative medicine $(\mathrm{CAM})$ is generally paid for by the user, but some CAM treatments (e.g., acupuncture, chiropractic, and osteopathy) are subsidized by the ACC if patients are eligible for ACC (Cumming et al., 2014). Low-income and high health service users are eligible for additional subsidies.

Approximately one-third of the New Zealand population hold private insurance to help cover co-payments, elective surgery, and specialist consultations (WHO \& Ministry of Health, 2012). Patients with private insurance are able to avoid long waiting times for the publicly funded healthcare; thus, long waiting times for the public services have led to growth in the private insurance market in New Zealand (Cumming et al., 2014). Despite free healthcare at public services and relatively low out-of-pocket patient payments compared to other OECD countries (OECD, 2013), high user healthcare charges for primary care and elective care are considered a cost barrier to accessing care, and it was one of the reasons for patients' 
unmet needs in New Zealand (Ministry of Health, 2014a). According to the Commonwealth Fund data, adults aged 65 or older reported that they did not follow their doctor's treatment recommendation or did not visit doctors due to the medical cost (Osborn \& Moulds, 2014). If the medical cost is the barrier to care, it would be possible that it may result in increased utilisation of ED (emergency departments) services because of its free services and the increased emergency medical care needs due to deferred primary care (Jatrana \& Crampton, 2009).

While patients in Korea freely choose their healthcare providers and facilities without booking and referrals, patients in New Zealand have to visit a GP first irrespective of their healthcare preferences and referral is a prerequisite to see a specialist. The cost of primary care in Korea is relatively low due to the highly competitive medical environment, whereas patients' access to primary care in New Zealand is often hindered by its high cost. However, healthcare at the secondary and tertiary levels that are provided by public healthcare services are free in New Zealand, and all accident related injuries are also free because they are covered by the ACC. Conversely, patients, who have freedom and various healthcare options as consumers in the commercially oriented healthcare system in Korea, pay for their healthcare that they have chosen depending on the type of care and the level of insurance coverage. The absence of the no-fault compensation system in Korea where the cost for the accident-related care is determined by the degree of culpability for the accident means that Koreans' freedom in healthcare is not free.

\subsection{Chapter summary and thesis overview}

This research aimed to build a theory that explains the main concerns of Korean immigrants with respect to their health and healthcare in New Zealand, and to shed light on Koreans' health-seeking behaviours and their healthcare adapting process within the New Zealand healthcare system. Chapter one provided the rationale and justification for the research and outlined the research purpose, question and objectives. This chapter also presented background information to provide an understanding of Korean immigrants in New Zealand. The four relevant areas of background information introduced were Koreans' migration history, the 
immigration purposes and settlement issues of Korean immigrants in New Zealand, and the healthcare systems in Korea and in New Zealand.

While Korea achieved significant economic growth during the past 60 years, the migratory movement in Korea also experienced transitions in this period. Koreans, who previously immigrated to foreign countries mainly for economic reasons, today immigrate to have a better life quality; and Koreans who immigrated to New Zealand are no exception. Despite their reason for immigration, the problematic settlement issues around them (such as limited English proficiency, low income, employment problems, and a lack of integration into a new society) have been noticed. To understand the main concerns of Korean immigrants in healthcare and their healthcare adapting process in New Zealand, the overview of the healthcare systems in Korean and in New Zealand including the comparison of the most notable differences between these systems are provided. The role of the healthcare system in healthcare acculturation and its influences on immigrant's health-seeking behaviour will be examined and discussed in Chapter two (Literature review) and in Chapter five (Findings).

In Chapter two I present a review of the literature relevant to the research findings. The purpose of this review is to support the research findings as data in order to complete the theory, not to demonstrate a research gap in the existing knowledge. In keeping with grounded theory methodology, the literature review, which was conducted after the data collection and data analysis, focuses on the association between Koreans' health-seeking behaviours and the affecting factors revealed in the research findings. I examine the literature on "migration", "acculturation", "ethnic identity", "immigrants' health service utilisation", and "health-seeking behaviours", including those of Koreans; and "illness perceptions of immigrants", "patients' satisfaction", “quality of healthcare”, and "person-centred care".

Chapter three demonstrates methodological choices and challenges I encountered throughout the research process. The chapter begins with the fundamental characteristics of qualitative research, followed by a history of grounded theory and its divergence. The overview of grounded theory methodological 
approach includes its essential tenets, such as the concurrent, iterative and integrative data collection and data analysis, and the constant comparative analysis together with theoretical sampling. I also discuss the challenges in adopting grounded theory that researchers may experience such as choosing one version from the various versions of grounded theory, the different roles of the literature review, and unfamiliar attributes of grounded theory as a general methodology.

The detailed theory building procedure conducted in this research is described in Chapter four. I provide detailed data collection and analysis procedures including the research setting, recruitment, participant's characteristics, different types of data used, and coding procedures. I describe the challenges and learning I experienced in conducting a grounded theory research in a second language. The ethical considerations associated with the participants and considerations of the quality of research in classic grounded theory are discussed in detail.

Chapter five introduces the substantive theory that emerged from the research, namely the Healthcare Acculturation Theory, and provides a detailed explanation of the process of theory development. The findings are discussed in light of the literature reviewed after data analysis. Korean immigrants' health-seeking behaviours and their transition pathways in the process of healthcare acculturation in New Zealand are presented with a series of figures in this chapter.

The concluding chapter commences by discussing the contribution this thesis makes to an understanding of Korean immigrants' health-seeking behaviour in New Zealand and its contribution to methodology and methods. Several implications from the findings of this research are introduced, and the research limitations that I perceived are presented in this chapter. Based upon these limitations I propose recommendations for future research. The chapter ends with concluding thoughts. 


\section{Chapter 2. Literature review}

\subsection{Introduction}

The role of the literature review and when it is conducted in grounded theory differs to those of other research methodologies, and is even diverse within grounded theory studies (Andrew, 2006). Delaying the literature review until completing the data analysis is encouraged in grounded theory in order to prevent the influence of the literature on an emerging theory (Chen \& Boore, 2009; Markey, Tilki, \& Taylor, 2014). The role of literature review in grounded theory is to compare with and to support the findings of the research, not to find a research gap in knowledge (Chen \& Boore, 2009; Ng \& Hase, 2008). Therefore, researchers in grounded theory analyse the relevant literature as another form of data in order to saturate their findings and articulate the theory emerged from the data (Charmaz, 2014). An in-depth discussion of the use of literature in grounded theory studies is provided in Chapter three, under the Section 3.5.4 Literature review: When and how.

There were two different literature reviews conducted for this research. I briefly reviewed literature to establish a gap in the area of Korean immigrants' health-seeking behaviour in New Zealand in order to complete my research proposal at the early stage of the research. A second literature review was undertaken as theory emerged from the data analysis to fill the gap that was addressed at the earlier literature review and to develop new theoretical knowledge. This chapter presents the latter literature review. Some of the earlier review, relevant to the research findings, is included. This literature review focuses on the association between immigrants' health-seeking behaviours and the contributing factors as revealed in the research findings. To understand immigrants, literature was examined that related to "migration", “acculturation", and "ethnic identity". To understand immigrants' health, I reviewed the literature on immigrants' health service utilisation and healthseeking behaviours including those of Koreans, and illness perceptions of immigrants. Lastly, to figure out why and how certain factors (findings from the research) affect Koreans' health-seeking behaviours, the literature relevant to patients' satisfaction, quality of healthcare and person-centred care was investigated. 
The keywords used for the search included: health-seeking behaviour, immigrants' health, healthy immigrant effect, health service utilisation, immigrants' health service utilisation, healthcare system, illness perception, migration, migration theory, acculturation, acculturation strategy, culture, ethnicity, ethnic identity, patient satisfaction, quality of health service, unmet need, healthcare expectation, and person-centred care.

\subsection{Migration}

Globalisation has brought greater interaction between countries; particularly, technological revolution in transportation and communication has facilitated and increased international migratory movement (Czaika \& de Haas, 2014; Kahanec \& Zimmermann, 2008). The interconnectedness of the world has accelerated and introduced greater diversity (Held, 1999). According to the United Nations Department of Economic and Social Affairs (UN-DESA), there were approximately 232 million international immigrants in the world in 2013 (an estimated 3.2\% of the world population); this number rose from 175 million in 2000 and is expected to reach 400 million by 2050 (United Nations Population Division, 2013). More than $50 \%$ of the world's migrants (117 million) resided in OECD countries in 2013 (estimated 10\% of OECD population) (OECD/European Union, 2015). In the 21st century, increased human mobility has become one of the most influential attributing factors of globalisation (Postelnicu, 2013).

"Migration" is often referred to as a geographical movement of individuals or groups of people including temporary and permanent movement; it covers both phenomena of emigration (leaving) and immigration (coming), and national and international migration (Hagen-Zanker, 2008; Kumpikaite \& Zickute, 2012). Unlike internal (national/domestic) migrants, migrants who move from country to country are generally regulated by international and receiving countries' migration policies, and are expected to experience cultural change as a result of crossing borders (Castles, 2000; Zohry, 2005). Hereafter migration and migrants will refer to "international migration" and "international migrants" as this research was focused on international migration. 
There are a variety of types of migrants depending on the purposes of migration, duration of stay, and legal status. Types include temporary and long-term labour and business migrants; undocumented or illegal migrants; refugees and asylum seekers; forced migrants as a result of famine/natural disasters or political issues; family reunion migrants; students; and returning migrants (Castles, 2000; OECD, 2006). These various types of migrants, in terms of migration categories, are ascribed to the globalised world and technological revolution, while the migration patterns and dynamics are reflected by global social, economic, political, environmental, and demographic situations (Castles \& Miller, 2009; Vertovec, 2007). For example, the huge influx of refugees from the Mediterranean countries (such as Syria, Afghanistan, Kosovo, Iraq, and Albania) to Europe has changed the migratory patterns in Europe over the past year ("Migrant crisis: Migration to Europe explained in graphics," 2015). Syrian refugees, who have fled from the civil war in their home country, have become the world's single largest refugee group (Carroll \& Qiu, 2015). According to the United Nations High Commissioner for Refugees (UNHCR) (2016), more than 4.6 million Syrians are living as refugees. As a result, Syrian refugees' legal and illegal displacement into Europe including their neighbour countries (Turkey, Lebanon and Jordan) increased public attention and raised the pressure on migration policies (UNHCR, 2015). Future migration policies in these countries are likely to be affected by this change.

Unlike voluntary migrants whose migration is based on their own decisions "for a better life" (de Haas, 2010c, 2014b; Thieme, 2006), forced migrants such as refugees and asylum seekers migrate as a matter of survival, from war or persecution. Nevertheless, the underlying assumption is, people will migrate continuously as long as inequalities (in terms of economic, demographic, social, and political conditions) remain between countries (Castles, 2000; Martin, 2013).

\subsection{Theories of migration and implications}

Since migration has become one of the global issues, various migration theories and theoretical models have emerged to explain the dynamics of migratory movement, and to address the causes and impacts of migration (Vieru, 2013). 
However, there is no single migration theory or model explaining all aspects of the migration phenomenon comprehensively; it is rather somewhat segmented (Baycan \& Nijkamp, 2013; Kumpikaite \& Zickute, 2012; Massey et al., 1993). The nature of migration itself, which is complex and multi-faceted, is often attributed as the main reason for the absence of comprehensive migration theories (Bijak, 2006; Castles, 2010; de Haas, 2008; King, 2012; Kumpikaite \& Zickute, 2012; Van Hear, 2010).

Migration is linked in complex ways to class, gender, generation, ethnicity and other social cleavages, which are embodied in hierarchies of power and social status, in positions in home and host communities, and in work and domestic relationships - all of which may be transformed in the course of the migratory process. (Van Hear, 2010, p. 1531)

Accelerated human mobility as a result of globalisation has contributed to increased volume and broadened geographical scope of migration; consequently, the migration phenomenon has become more complex and diverse than ever (Baycan \& Nijkamp, 2013; Czaika \& de Haas, 2014). Sociologist Massey and his colleagues (1993) proposed incorporation of various segmented migration theories and approaches in order to provide a more complete picture, whereas de Haas (2008), who is also a sociologist, argued that employing different migration theories together would be problematic because individual migration theories and approaches are underpinned by different theoretical paradigms and with different methodological criteria. A number of migration researchers have agreed that it would be impossible to develop a single general theory capturing the entire phenomenon of migration because of its complex and diverse nature (Baycan \& Nijkamp, 2013; Borjas, 1989; King, 2012; Kumpikaite \& Zickute, 2012; Massey et al., 1993; Thieme, 2007).

In literature, migration theories are often categorised by certain distinctions amongst them such as "stages of migration" or "levels of migration analysis" (Hagen-Zanker, 2008; Massey et al., 1993; Thieme, 2007). For example, the theories are divided into two sub-categories depending on the interests of migration; "initiation" of migration theories and "perpetuation" of migration theories. The 
theories categorised by the levels of migration analysis are "micro-, meso-, and macro-level" "9 theories.

\subsubsection{Theories of migration initiation}

Most early migration theories and approaches were developed to explain the initiation (drives) of migration with focus on international labour migration (Jennissen, 2007; Massey et al., 1993; Thieme, 2007). These theories emphasise economic factors in migration, and assume discrepancies in economic conditions (levels of wages and employment opportunities) between countries as the main drives of migration.

Push-Pull Model (Lee, 1966) which originated from Ravenstein's (1885) "Law of migration" is one of the popular approaches on the migration initiation theories (Kumpikaite \& Zickute, 2012; Wang, 2010). Geographer Ravenstein formulated his theory based on observation of human behaviour through the census data from England and Wales (Grigg, 1977; Wang, 2010). He viewed geographical economic disparity as a primary reason for migration, and concluded that the dynamics of migration were directly determined by push-pull factors (Greenwood \& Hunt, 2003; Wang, 2010). Later Lee (1966), a demographer, proposed a new analytical framework Push-Pull Model based on Ravenstein's theory (de Haas, 2008). Lee (1966) highlighted four key factors that determine migration decisions: push (minus) factors which are associated with the area of origin; pull (plus) factors which are associated with the area of destination; intervening obstacles between two areas (origin and destination), such as distance and cost of moving; and personal factors including individuals' knowledge, information, and perceptions regarding migration. According to the Push-Pull Model, migration decisions are affected by

\footnotetext{
${ }^{9}$ While micro-level migration theories emphasise individual factors of migration, such as individuals' wages, skills, and behaviours, and propose that migration decisions are largely influenced by individuals' cost-benefit calculations; theories on macro-level perspective concern national and global migration flows, and focus on global structural factors, such as international economic, political and social circumstances (Hagen-Zanker, 2008; Massey et al., 1993; Morawska, 2007). Unlike theories of macro- and micro-level perspectives, which focus on causes of migration, theories of meso-level concern perpetuation/continuation of migration; how migration is maintained and continues (de Haas, 2010a, 2011; Faist, 2009). Meso-level migration theories focus on individuals' social ties (kinship, ethnic and community ties, and friendship) and networks rather than economic factors of migration.
} 
comparisons of the push factors (minus factors: low wages, high unemployment rates, heavy taxation, and lack of healthcare) and the pull factors (plus factors: high wages and high employment rates) of both origin and destination. In this regard, migration is likely to occur if the pull (plus) factors at the destination outweigh that of the origin. While this model has been broadly employed and gained popularity, it has also been subjected to criticism (Kumpikaite \& Zickute, 2012). Hagen-Zanker (2008) and de Haas (2010c, 2014b) point out the overly simplified push and pull factors in the model fail to provide exact causal mechanisms behind the migration flow of why individuals and groups of people move. They claim that the heterogeneity of migration is ignored in this model. In addition, the model does not provide an explanation of how the intervening obstacles and different roles in gender enter into the equation.

In the Neo-classical Economic Migration perspective, migration occurs mainly due to a discrepancy in economic factors between countries such as different wages and employment conditions (de Haas, 2010b; Jennissen, 2007). In general, countries with a high labour demand have a relatively high wage, while countries with a high labour supply have a relatively low wage, and migrants tend to move from low-wage to high-wage countries (Jennissen, 2007); migration is an individual investment decision for maximised benefit based on cost and benefit calculations. The Neo-classical Economic Migration theorists assume migration as a part of economic development, and consider individuals as a main contributing factor in migration decisions (micro level) (Flatau, 2002; Harris \& Todaro, 1970; Morawska, 2007). Individuals' migration decisions are significantly determined by human capital, such as age, gender, levels of education, skills, and experiences (Baycan \& Nijkamp, 2013; de Haas, 2008; Kumpikaite \& Zickute, 2012; Morawska, 2007). Meanwhile, individualistic migration assumptions of Neo-classical Economic Migration have been criticised by the New-economics of Labour Migration (NELM) perspectives (Castles, 2010; de Haas, 2010b; Kurekova, 2011; Massey et al., 1993).

NELM theorists argue that migration decisions involve members of families and households rather than the individual alone, and the purpose of migration is not only to maximise income, but also to minimise risks on families and households (as 
known as a risk spreading strategy). Thus, the migration decision takes into account security of employment (labour insurance) and availability of market capital in order to manage tentative risks, besides wages differences and employment opportunities (Baycan \& Nijkamp, 2013; Thieme, 2007; Xhaferaj, 2014). The concern according to de Haas (2010b) is that, these security conditions are not achievable for all immigrants because some markets do not have these security systems, and they may be available only for elite groups even if they exist. This NELM theory has been recognised as more realistic and useful than the Neo-classical Economic Migration Theory, but its migration assumption is still heavily focused on economic factors, and considers these as the main reasons for migration (Castles, 2010; de Haas, 2014a).

Unlike theories of migration above (Push-Pull Model, Neo-classical Economic Migration Theory, and NELM theories), Dual (segmented) Labour Market Theory views migration at a global (macro) level, and emphasises labour demand in industrial societies as a key contributing factor for migration (Massey et al., 1993; Piore, 1979). According to this theory, the labour markets in developed countries are segmented into two sectors: the primary sector with high-skilled and well-paid jobs, and the secondary sector with low paid unskilled jobs (Baycan \& Nijkamp, 2013; Jennissen, 2007; Kumpikaite \& Zickute, 2012). While jobs in the primary sector attract the local population and are filled by them, the secondary sector experiences shortage of labour; consequently, labour shortage in the secondary sector motivates international labour migration. Given this perspective, the flow of labour migration tends to move from less developed countries to more developed countries.

Similarly, the World Systems Theory takes global level perspectives (macro), and assumes that migrants generally move from economically poor countries to economically rich countries (Hagen-Zanker, 2008; Jennissen, 2007; Massey et al., 1993). The global migratory movement, which is a result of interactions between countries and structural changes in the global economy, is a part of the capitalist development process (Hagen-Zanker, 2008; Jennissen, 2007; Kurekova, 2011; Massey et al., 1993). However, Hagen-Zanker's (2008) critique is that circumstances in sending countries (push factors) were disregarded in the World Systems and Dual 
Labour Market theories, while receiving countries' circumstances (pull factors) were heavily considered. Theories of migration initiation shed light on the global labour migration; however, the various causes of migration within these theories are simply attributed to the economic factors of migration which were isolated from the other aspects of migration, such as social, cultural, political, demographical, and environmental factors (Bodvarsson \& Van den Berg, 2013; Thieme, 2007).

\subsubsection{Theories on perpetuation of migration}

Theories on the perpetuation of migration focus on how migration sustains itself across time and space, rather than the causes of migration (de Haas, 2010a; Massey et al., 1993). Therefore, the factors determining the continuous nature of migration such as social ties and networks, institutional supporting, and transnational activities (meso-level) are major concerns for these theories (de Haas, 2010a; Massey et al., 1993; Thieme, 2007).

Network Theory emphasises migrants' interpersonal connection as the most important contributing factor for perpetuating migration (Kumpikaite \& Zickute, 2012; Thieme, 2007). For example, family, relatives, friends and acquaintance ties, and these social ties (also known as social networks) play a role as resources (capital) in migration life (Bijak, 2006; Bodvarsson \& Van den Berg, 2013; Castles, 2010; Kumpikaite \& Zickute, 2012). Migrants with strong networks usually have more advantages in their migrant lives; for example, information from their networks would help them to find jobs or find places to live, and these networks would be of crucial help to them opening new businesses and managing them (Baycan \& Nijkamp, 2013; Kumpikaite \& Zickute, 2012). The advantages of migration network reduce not only economic risks for individuals but also social and psychological risks (Bijak, 2006; de Haas, 2010a; Kumpikaite \& Zickute, 2012; Massey et al., 1993; Thieme, 2007). As a result, people's migration becomes easier and more secure; consequently, migration is more likely to be continued. The expansion of migration network can result in increased motivation of migration including additional migration in both migrant and non-migrant groups (de Haas, 2010a; Massey et al., 
1993). In Network Theory, migrants' network is considered as a collective agency of migrants (Castles, 2010).

Similar to the Networks Theory, the Cumulative Causation Migration Theory emphasises migration development, and provides an idea of how migration develops and is continuous (de Haas, 2008; Kumpikaite \& Zickute, 2012; Kurekova, 2011).

These researchers believe that migration has a tendency to develop its own social and economic infrastructure, which helps migration to perpetuate itself (Baycan \& Nijkamp, 2013; Massey, 1988, 2003). For example, individuals who migrated due to their networks (encouraged by information including lowered costs and risks of migration) are likely to motivate others with their own migration experiences. This means that once migration takes place, the cumulative causation process starts, and migration is able to sustain itself due to its cumulated causation; and migration is expected to increase as well (Massey, 1988, 1999; Massey et al., 1993).

A concept of "transnationalism" has been employed by migration researchers to describe the contemporary transnational migration trend (Baycan \& Nijkamp, 2013; Kurekova, 2011). As a result of globalisation (reduced traveling time and cost, and ease of communication), human mobility has been spurred, and migration has become more broad, diverse, and complex (Baycan \& Nijkamp, 2013; Kurekova, 2011). However, the connection between migrants and their home societies has become closer than ever (Baycan \& Nijkamp, 2013; Castles \& Miller, 2009; Czaika \& de Haas, 2014; Kurekova, 2011). According to Portes, Guarnizo and Landolt (1999), transnational migrants are those:

who live dual lives: speaking two languages, having homes in two countries, and making a living through continuous regular contact across national borders. Activities within the transnational field comprise a whole gamut of economic, political and social initiatives ranging from informal import-export businesses, to the rise of a class of binational professionals, to the campaigns of home country politicians among their expatriates. (pp. 217-218)

Previously, migration was commonly accompanied by disconnection from the home countries: for example, disconnection from family members, friends, social 
network, and their supports (Suårez-Orozco, Todorova, \& Louie, 2002). However, the transnational migration trend affected and changed the dynamics and patterns of migration; as a result, the previous migration theories were challenged, and the transnational migration approaches were developed to explain the new trend of migration (Baycan \& Nijkamp, 2013; de Haas, 2010b; Kurekova, 2011). The transnational migration approaches focus on transnational social spaces of migrants, and emphasise their transnational characteristics of migration, such as transnational networks and ties, and cross border activities between both the host and home countries (Faist, 2000; Kurekova, 2011; Vertovec, 2004). According to Baycan and Nijkamp (2013), migration should be understood within a global context (transnationalism), rather than a national context (nationalism).

Migration studies should focus on the evolving migration process, rather than a static snapshot; further, researchers should analyse migration based on the complex, mixed and shifting motivations of migrants, not by simple categories and dichotomies (e.g., "origin" or "destination"; and "permanent”, "temporary", or "return" migration). Since the nature of migration is multi-faceted, migration could be better understood through interdisciplinary approaches rather than methodological approaches. The transnational migration trend has become a part of the global transformation processes, and the approaches of transnational migration have changed the migration perspectives in migration studies (Baycan \& Nijkamp, 2013; Lee, Carling, \& Orrenius, 2014).

\subsection{Acculturation}

As a consequence of migration, migrants who come from various cultural backgrounds are exposed to new cultures in the host societies; and when they engage in intercultural contact, the process of acculturation takes place (Berry, 2005; Sam \& Berry, 2010). The concept of acculturation is referred to as the process of cultural adaptation or cultural learning with individuals experiencing various changes including not only cultural changes but also changes in values, attitudes, and behaviours (Berry, 1997; Organista et al., 2010). However, the acculturation attitudes of individuals, which have been reported in the literature, are not the same even if 
they share the same cultural heritage (Berry, 2005; Sam \& Berry, 2010; Schwartz, Unger, Zamboanga, \& Szapocznik, 2010). Acculturation studies have generally focused on how individuals engage the acculturation process, what factors affect their acculturation, and what effects and to what extent they are affected by these effects (Sam \& Berry, 2010).

Early acculturation studies were predominantly undertaken by anthropologists and sociologists, and different terms such as "assimilation" and "incorporation" were often adopted to refer to acculturation (Lakey, 2003; Organista et al., 2010). Within their perspectives, migrants are expected to assimilate or incorporate into the host society as can be seen from the terms above (Organista et al., 2010; Schwartz et al., 2010). The cultural transitions amongst migrants occur in one direction from the heritage culture to the host culture, and giving up one's own culture is a prerequisite to acquiring the host culture (Gordon, 1964). This means that acculturation is a unidimensional process. Unlike sociologists and anthropologists, psychologists view acculturation not just as a unidimensional process (Berry, 1997; Organista et al., 2010; Ryder, Alden, \& Paulhus, 2000; Schwartz et al., 2010). They argue that gaining a new culture is not necessarily followed by losing the heritage culture; migrants not only acquire a new culture, but can also retain their heritage culture. Psychologists emphasise bi/multi-dimensional acculturation approaches and migrants' multi-cultural identities. They criticise the over-simplified and incomplete conceptualisation of acculturation in unidimensional approaches.

According to psychologists, the acculturation of individuals is not the same because acculturation is influenced by values, beliefs, attitudes, and characteristics of individuals such as ethnicities, gender, age, socioeconomic status, education levels, language proficiency, and health status (American Psychological Association, 2012; Berry, 2005; Sam \& Berry, 2010). Psychologists emphasise the role of individuals who have choices in acculturation; individuals make a decision to acculturate whether to acquire the host culture or not, and whether to retain or leave behind the heritage culture (Lakey, 2003; Organista et al., 2010; Ryder et al., 2000; Schwartz et al., 2010). The changes in values, beliefs, and behaviours of acculturating individuals take place as a consequence of their acculturation choices. Psychologist Berry (1997, 
2003, 2005) conceptualised acculturation with two separate processes: a cultural process and a psychological process. A cultural process is a group level cultural change that results from intercultural contact. This change is accompanied by social structural and institutional changes, and cultural practice changes. While a cultural process takes place at a group level, a psychological process occurs at an individual level, and the change involves individuals' attitudinal and behavioural aspects.

A well-known bi-dimensional acculturation model was developed by Berry. According to his model, acculturation occurs in two dimensions/directions, which involve retaining or discarding the heritage culture and/or acquiring or rejecting the host culture. He proposed four acculturation strategies based on his two-dimensional acculturation assumption, which can be adopted by individuals as acculturation approaches: "integration", "assimilation", "separation”, and "marginalisation". The integration strategy engages both adopting a new culture and retaining the heritage culture, and assimilation strategy refers to acquiring the host culture but rejecting the heritage culture. Conversely, separation strategy rejects the host culture while maintaining the heritage culture, and marginalisation strategy discards both the home and host culture. These four strategies demonstrate different responses to acculturation, and the strategy adopted by an individual reflects their acculturation orientation including the level of engagement with the heritage and host cultures (Organista et al., 2010; Ruzek, Nguyen, \& Herzog, 2011).

Berry's model of acculturation has been widely accepted and gained popularity in acculturation literature. The four acculturation strategies, which affirm individuals' different acculturation choices, are recognised as one of Berry's major contributions to acculturation studies (Organista et al., 2010; Schwartz \& Zamboanga, 2008). However, there are some criticisms of his model related to the four strategies. Particularly, marginalisation (one of the four strategies) has been criticised for its reliability and validity (del Pilar \& Udasco, 2004; Matsudaira, 2006; Organista et al., 2010; Schwartz et al., 2010; Schwartz \& Zamboanga, 2008). Individuals who adopt the marginalisation strategy reject both the home and host cultures (Berry, 1997), but the question is can an individual remain cultureless? In addition, according to a number of researchers, Berry's four acculturation strategies 
are not sufficient to cover immigrants' diverse ways of acculturating because they are overly generalised and simplified (Kim \& Omizo, 2006; Organista et al., 2010; Padilla, 2006; Ruzek et al., 2011; Schwartz \& Zamboanga, 2008). For example, individuals who have two or more cultural identities (i.e., children from intermarriage or second-generation migrants) are not able to fit into any of the four strategies (Organista et al., 2010).

Schwartz and Zamboanga's study (2008) examined Berry's (1997) acculturation model with 436 Hispanic college students in the USA, and found that more than the four strategies of acculturation were adopted by the participants. In the study, Berry's integration (biculturalism ${ }^{10}$ ) category appeared to have two groups: "separation and biculturalism" and "assimilation and biculturalism". Another study (Arends-Tóth \& Van de Vijver, 2007) which focused on acculturation attitudes of Turkish immigrants in the Netherlands, also demonstrated that some groups of immigrants did not fit into any one of Berry's four strategies. The study found that some Turkish immigrants employed two different acculturation strategies and used them separately. For example, some participants adopted the strategies of separation and integration to acculturate to the host society; the integration strategy was adopted for the public domains ${ }^{11}$, and the separation strategy was employed for the private domains ${ }^{12}$. Arends-Tóth and Van de Vijver's study result is supported by Miller and Lim's (2011) domain-specific nature of acculturation strategy hypothesis. According to Miller and Lim, acculturating individuals adopt different strategies and engage different levels of acculturation depending on the acculturation domains.

A number of researchers have stressed that the acculturation process is affected by individuals' demographic and contextual factors (Bhatia \& Ram, 2001;

\footnotetext{
${ }^{10}$ Biculturalism broadly refers to cultural integration of behaviours, values, beliefs, and customs in both the heritage and mainstream cultures (Schwartz \& Unger, 2010; Schwartz et al., 2010). Bicultural individuals acknowledge their dual cultural identities and practice both cultures simultaneously. Biculturalism reflects the integration category, which is one of the four strategies in Berry's model.

${ }^{11}$ Public domains referred to "social contacts, friends at school, speaking the language, reading the language, education and courses, teachers, news and information, and reading newspapers" (Arends-Tóth \& Van de Vijver, 2007, p. 1475).

12 Private domains included "celebrations, eating food, child-rearing practices, cultural habits, cultural way of life, self-reported cultural identity, and attributed cultural identity as seen by the respondent" (Arends-Tóth \& Van de Vijver, 2007, p. 1475).
} 
Organista et al., 2010; Rudmin, 2003; Schwartz, Montgomery, \& Briones, 2006; Schwartz et al., 2010). Some researchers argue, it would be difficult to acculturate if the existing cultural gap between the origin and host country is too big (Bhatia \& Ram, 2001; Rudmin, 2003; Schwartz et al., 2006). For example, the acculturation process of individuals who came from English speaking countries immigrating to a same language country would be easier than moving to a non-English speaking country; and acculturating would be more difficult for the individuals who came from collectivist-oriented heritage cultures moving to Western, individualisticoriented cultures, and vice versa (Rudmin, 2003; Schwartz et al., 2010). In addition, acculturation of adults would be more difficult than that of young children, and acculturation would be less serious for the second-generation immigrants. This means, acculturation is likely to be considered more or less serious depending on the age of the immigrants and the generation of the immigrants.

Schwartz and his colleagues (2010) pointed to limitations of the one-size-fitsall approach in acculturation studies. They argue that the one-size-fits-all approach would generalise and simplify various characteristics of individuals in order to adjust to the approach. They criticised the researchers employing a one-size-fits-all approach (which is popular or widely used, for example Berry's four acculturation strategies) without considering various characteristics and types of immigrants. In this regard, it has been suggested that if researchers analysed the acculturation phenomenon in a flexible manner rather than simply applying the one-size-fits-all approach, it would provide a better understanding with an applicable explanation (Chirkov, 2009; Schwartz et al., 2010).

\subsection{Ethnic identity in acculturation}

In culturally plural societies, immigrants are often described by their ethnic identities, and these identities help to distinguish one ethnic group from another (Matsunaga, Hecht, Elek, \& Ndiaye, 2010). While immigrants are labelled by their ethnic identities according to race, culture, and language (who they are), cultural identity explains what their ethnic identity means as shown in their beliefs, values, and traditions (Hall, 2004; Nagel, 1994; Ravanera \& Fernando, 2009). Ethnic 
identities are more likely inherited, but can be redefined and reconstructed (Ravanera \& Fernando, 2009). According to Ravanera and Fernando, when immigrants are exposed to culturally diverse contexts, their sense of ethnic identity emerges to identify themselves with the other ethnic groups, and their social identities (such as cultural and national identity) are constructed and/or reconstructed during the process of acculturation (Berry, 2004). Identities of immigrants imply their sense of belonging or attachment to a certain group or culture (Phinney, 1990; Phinney \& Ong, 2007).

Many studies have investigated the association between ethnic or cultural identities of immigrants and their acculturation outcomes; and a number of these studies have confirmed that positive acculturation outcomes were generally positively associated with a well-developed and strong sense of ethnic identities (Berry, 2003; Lopez, 2008; Schimmele \& Wu, 2015; Schwartz et al., 2010). For example, a meta-analysis study, which examined the relationship between ethnic identity and well-being of non-whites in North America, found that strong ethnic identity of individuals contributed to individuals' well-being (Smith \& Silva, 2011). Some empirical studies also indicated immigrants' better psychological conditions such as increased self-esteem as a result of positive ethnic identities (Lopez, 2008; St. Louis \& Liem, 2005; Umaña-Taylor, 2004), and decreased depression and anxiety (Williams, Chapman, Wong, \& Turkheimer, 2012). Further, according to the studies focusing on the perceived discrimination of ethnic minorities, individuals with a high level of positive ethnic identity are less likely to be influenced by discrimination (Noh \& Kaspar, 2003; Pérez, Fortuna, \& Alegria, 2008; UmañaTaylor, Wong, Gonzales, \& Dumka, 2012). Individuals who have a well-developed sense of ethnic identity are also less likely to be involved with substance use; for example, alcohol and drugs (Kulis, Marsiglia, Kopak, Olmsted, \& Crossman, 2012; Love, Yin, Codina, \& Zapata, 2006).

While evidence from the most robust studies have emphasised the benefits of immigrants' strong ethnic identities, the concern is whether immigrants' sense of belonging to the host country is impeded by these identities (Costigan \& $\mathrm{Su}, 2004$; Ryder et al., 2000; Schimmele \& Wu, 2015). Noels, Pon, and Clément (1996), who 
examined the cultural orientation between the heritage and host cultures of Chinese university students in Canada, discovered a correlation between ethnic identity and a sense of attachment to the host culture; a stronger sense of Chinese identity resulted in a lowered level of involvement with Canadian culture. However, according to Ravanera and Fernando (2009), the relationship between immigrants' ethnic identities and their senses of belonging to the host society are compatible.

Ravanera and Fernando's study examining young people's (aged 15-24) attachment to the host society from five ethnic groups (Non-visible minority, Chinese, South Asians, Blacks, and Other visible minority ${ }^{13}$; the data from the Ethnic Diversity Survey [EDS, 2002] in Canada) found that 70\% of the 7,497 participants who self-identified their own ethnic group also demonstrated a strong sense of attachment to Canada. Likewise, about $20 \%$ of the participants who classified themselves as Canadian did not show a strong sense of belonging to Canada. A number of researchers and studies have confirmed that immigrants' strong ethnic identities do not hinder their sense of belonging to the host society (Berry, Phinney, Sam, \& Vedder, 2006; Costigan \& Su, 2004; Kiang, Yip, \& Fuligni, 2008;

Schimmele \& Wu, 2015). Costigan and Su's (2004) research on immigrants' cultural engagement between the origin and host cultures also found that immigrants' cultural practice of the heritage and the host culture were not interrelated; they were not affected by each other. Individuals who participate in two different cultures (the heritage and host cultures) generally simultaneously develop bicultural identities and bicultural orientations, and are often described as bicultural individuals (Berry et al., 2006; Schimmele \& Wu, 2015).

Individuals with bicultural identities have been found to show better adjustment in the process of acculturation, as biculturalism refers to the most successful and positive adaptation strategy (as known as integration strategy) amongst the four acculturation strategies (Berry, 1997; Chen, Benet-Martínez, \& Harris Bond, 2008; Schwartz et al., 2006). Although bicultural individuals are

\footnotetext{
${ }^{13}$ According to Statistics Canada, "visible minorities" refers to "persons, other than Aboriginal peoples, who are non-Caucasian in race or non-white in colour". These minorities in Canada are Chinese, South Asian, Black, Arab, West Asian, Filipino, Southeast Asian, Latin American, Japanese, and Korean (Statistics Canada, 2015).
} 
attached to two different cultures, they tend to develop a personalised culture which is found to somewhat differ from their original heritage and their host culture as a result of engaging two different cultures in a certain amount of time (Benet-Martínez, Leu, Lee, \& Morris, 2002; Schwartz \& Unger, 2010). Besides, the traditional concept of ethnic identity has been challenged against the concepts of bicultural identity, global identity, multicultural identity, or intercultural identity ${ }^{14}$ (Kim, 2006, 2008, 2009b; Kunst \& Sam, 2013; Moore \& Barker, 2012).

In a globalised world, the concept of identity has become more fluid, flexible, and multifaceted beyond the traditional ethnic categories, territories and borders (Adler, 2002; Kim, 2008; Moore \& Barker, 2012; Scholte, 2005). Kunst and Sam's (2013) study of the acculturation process of ethnic minorities found a global identity amongst culturally marginalised individuals. While ethnic minority individuals with a stronger global identity showed higher levels of sociocultural adaptation, the ethnic minority individuals with lower levels of global identity displayed lower levels of sociocultural adaptation. According to the findings of Kunst and Sam's study, global identity is generally associated with positive outcomes of acculturation, such as lower levels of stress and higher levels of life satisfaction; and marginalised ethnic minority individuals with a strong global identity seem to have lives more connected to others rather than simply being isolated from the ethnic and host cultures (Kunst \& Sam, 2013). However, it is not well-defined whether marginalised individuals chose marginalisation ${ }^{15}$ as their acculturation strategy due to their lack of sociocultural competence or for their ideological reasons, and whether they chose a global identity as an alternative to replace the role of the ethnic and/or host identity or not.

A study by Moore and Barker (2012) also revealed multicultural identity in their participants who lived in a foreign culture during their developmental ages. Multicultural individuals, according to Moore and Barker "have integrated different elements from each culture, thus forming one multicultural identity to which they

\footnotetext{
${ }^{14}$ The concept of intercultural identity "is employed as a counterpoint to, and as an extension of, cultural identity, and as a concept that represents the phenomenon of identity adaptation and transformation beyond the perimeters of the conventional, categorical conception of cultural identity" (Kim, 2008, p. 359).

${ }^{15}$ See the Section 2.4 Acculturation (p. 30).
} 
adhere consistently regardless of the country, context, and culture they are in" (p. 599). They also call these multicultural identity individuals "third culture individuals". A concept of intercultural identity, developed by Kim (2008), views identity as a way of being to oneself and to the outside world, which is "open-ended, adaptive, and transformative" (p. 364).

Immigrants' identities evolve or change during the process of acculturation (Schimmele \& Wu, 2015) even though their host societies simply label them by their races or ethnicities (Matsunaga et al., 2010). As shown in the literature, immigrants with a well-developed sense of identity, whether it is an ethnic or a global identity, appear to have better acculturation outcomes (Berry, 2003; Kunst \& Sam, 2013; Lopez, 2008; Schimmele \& Wu, 2015; Schwartz et al., 2010). It is unclear how the acculturation outcomes of immigrants with a strong ethnic identity and with a strong global identity differ, and how these identities are interrelated and influence the outcomes of acculturation.

\subsection{Immigrants' health and acculturation}

Acculturation has also been associated with significant influences on immigrants' health and health behaviours (Kennedy et al., 2006; Sanou et al., 2014; Schwartz et al., 2010; Shin \& Lach, 2014). Immigrants' health is often explained or predicted by their degree of acculturation (Dressler, 1993; Hunt, Schneider, \& Comer, 2004) because their healthcare and health outcomes are directly related to their levels of language proficiency, length of stay, and legal and socioeconomic status in the host societies (Davies, Basten, \& Frattini, 2009; Derose, Escarce, \& Lurie, 2007; Dias, Severo, \& Barros, 2008; Lenz, Bauer-Dubau, \& Jelinek, 2006; Zimmerman, Kiss, \& Hossain, 2011). Although many empirical studies have revealed the strong association between immigrants' levels of acculturation and their health (Kennedy et al., 2006; Sanou et al., 2014; Schwartz et al., 2010; Shin \& Lach, 2014), the impacts of acculturation on health did not show a consistent positive or negative trend; rather findings were somewhat mixed or scattered (Lara, Gamboa, Kahramanian, Morales, \& Hayes Bautista, 2005; Lee, Chen, He, Miller, \& Juon, 2013). Thus, questions about immigrants' health pertaining to acculturation still 
remain to be answered. In particular, there is a need to understand how immigrants' health is affected by the process of acculturation; what effects acculturation has on their health; and to what extent their health outcomes are influenced by these effects (Salant \& Lauderdale, 2003; Schwartz et al., 2010).

In the literature, immigrants are frequently referred to as a "vulnerable population" because their health is easily exposed to risks; for example, the high proportion of delayed diagnosis and treatment, or inadequate medical care (Lenz et al., 2006). During the process of acculturation, immigrants encounter various challenges (e.g., cultural differences, language difficulties, and socioeconomic disadvantages), and experience life changes; consequently, changes in health outcomes appear as a result of acculturation (Davies et al., 2009; Zimmerman et al., 2011). Immigrants who emigrate from relatively less developed countries than the host countries, in particular, are expected to encounter more serious acculturation problems, such as low income, unemployment and underemployment, limited education opportunities, and discrimination (Pong \& Zeiser, 2012; Smokowski, David-Ferdon, \& Bacallao, 2009). In addition, the differences in health between immigrants and native-born citizens in terms of physical and psychological conditions, disease profiles, and beliefs of health and illness, have been reported (de Luca, Ponzo, \& Andrés, 2013). These health disparities could leave the host societies with a limited understanding or misunderstanding of immigrants and their health, and could hinder the reception of adequate and timely healthcare of immigrants (International Organization for Migration, 2013).

In spite of immigrants' vulnerability regarding their health, studies have found that immigrants tend to have better health than the native-born population and the more acculturated immigrants (Alegria et al., 2008; Baker, Rendall, \& Weden, 2015; Cunningham, Ruben, \& Narayan, 2008; Kim \& Chan, 2004; Lara et al., 2005; Pérez-Escamilla \& Putnik, 2007). For example, Cunningham et al.'s (2008) review of 71 studies on immigrant health in the USA (1980-2007), demonstrates that foreign-born immigrants are less likely to be diagnosed with heart disease, some cancers (breast, prostate, and colon), obesity, and mental disorders; and more likely to have lower mortality rates and better pregnancy outcomes than non-immigrant 
population. These findings have led to the concept of the "epidemiological paradox"; the better health outcomes of foreign-born immigrants compared to the dominant population, in spite of their marginal status (e.g., relatively low levels of income, less educated, lack of health insurance, and less use of health services) that is generally associated with worsened health outcomes (Alegria et al., 2008; Flores \& Brotanek, 2005). This phenomenon is commonly referred to as the "healthy immigrant effect", which is explained by the tendency of self-selection in immigration (young and healthy individuals are more likely to migrate), and the characteristics of national immigration policies (more favourable to young and healthy individuals) (Kennedy et al., 2006; McDonald \& Kennedy, 2004; Sanou et al., 2014).

The healthy immigrant effect is known to diminish gradually as immigrants' acculturation levels increase; in other words, the longer immigrants stay in the host country, they lose more health advantages and gain more health problems (Alegria et al., 2008; Antecol \& Bedard, 2006; Cunningham et al., 2008; Kennedy et al., 2006; McDonald \& Kennedy, 2004; Sanou et al., 2014; Schwartz et al., 2010). As a result, the health gap between immigrants and the non-immigrant population becomes smaller. With increased length of stay, losing health advantages has been linked to adopting the unhealthy host lifestyle and poor health behaviours including dietary habits (Ayala, Baquero, \& Klinger, 2008; Cunningham et al., 2008; Lara et al., 2005; Laroche, Kim, Tomiuk, \& Bélisle, 2005; Rubalcava, Teruel, Thomas, \& Goldman, 2008). For example, individuals who are more exposed to the host culture tend to consume more fast/instant food, and less fruit and vegetable (Ayala et al., 2008; Lara et al., 2005; Laroche et al., 2005). More acculturated individuals are more likely to engage in smoking, drinking, and other drug use than that of less acculturated counterparts (Chun \& Mobley, 2014; Corlin, Woodin, Thanikachalam, Lowe, \& Brugge, 2014; Rubalcava et al., 2008). Furthermore, acculturation increased the chances of obesity (Oza-Frank \& Cunningham, 2010; Park, Myers, Kao, \& Min, 2009), diabetes (O'Brien, Alos, Davey, Bueno, \& Whitaker, 2014), and hypertension (Wong, Dixon, Gilbride, Kwan, \& Stein, 2013).

Heterogeneity in health and health behaviours amongst immigrants is identified by Kennedy, Mcdonald, and Biddle (2006). According to these 
researchers, individuals who came from less developed countries were relatively healthier and had better health behaviours (e.g., healthy diet, low consumption of alcohol, tobacco, and drugs) compared to the host population, whereas immigrants who came from countries that were economically and culturally similar to the host country showed similar health statuses and behaviours to the native-born population. In this regard, it can be said that the healthy immigrant effect generally appears in immigrants who came from less developed countries to more developed countries with great cultural distance (Kennedy et al., 2006; Vang, Sigouin, Flenon, \& Gagnon, 2015).

While a number of studies have found that immigrants with longer duration or greater levels of acculturation are associated with poorer health conditions, and this is ascribed to adopting an unhealthy lifestyle from the host culture (Ayala et al., 2008; Cunningham et al., 2008; Lara et al., 2005; Laroche et al., 2005; Rubalcava et al., 2008); there is some empirical evidence against the negative impacts of acculturation on immigrants' health (Ro, 2014; Vang et al., 2015). For example, more acculturated individuals were positively associated with adopting some healthy behaviours like physical activities including exercise and leisure (Abraido-Lanza, Chao, \& Florez, 2005; Pérez-Escamilla \& Putnik, 2007); and have a lower likelihood of type 2 diabetes (Jaber, Brown, Hammad, Zhu, \& Herman, 2003; Pérez-Escamilla $\&$ Putnik, 2007). In a study that examined acculturation and the health of 517 Korean American adults (Shin \& Lach, 2014), it was found that Korean Americans' physical health was positively associated with their acculturation levels. Participants who adopted well into the host society demonstrated better physical health than other counterparts who were less acculturated in the study. Similarly, Eamranond et al. (2009), who investigated the association between the levels of acculturation and cardiovascular risks amongst Hispanic adults, found that cardiovascular risks tend to improve with acculturation; more acculturated Hispanics had lower blood pressure levels than Hispanics who were relatively less acculturated.

Most acculturating individuals encounter challenges and difficulties in adapting to a new culture and society, and experience various stressors due to these challenges and difficulties (Organista et al., 2010). Berry and his colleagues (1987) 
named this psychological stressful condition "acculturative stress" which is caused by conflicts between two cultures (origin and host). Individuals who have acculturative stress experience psychological distress, and exhibit feelings of depression, anxiety, and confusion depending on their stress levels. Berry (1997, 2003, 2006) analysed individuals' different levels of acculturative stress related to the four acculturation strategies ${ }^{16}$. According to Berry, individuals with the integration strategy (who chose to integrate both origin and host culture) experience the least stress during the acculturation process because they have more flexible and selective choices between two cultures. Conversely, individuals with the marginalisation strategy (who decided to discard both cultures) are expected to have the most stress. That is, immigrants' acculturative stress is influenced by the degree of acculturation; the more acculturated individuals have lower acculturative stress, and the less acculturated individuals have higher levels of acculturative stress. Berry considers the integration strategy (biculturalism) as the most desirable choice to acculturate due to its positive influences on health outcomes. However, Schwartz and Zamboanga (2008) argue that the bicultural individuals (individuals with the integration strategy) would have more stress than those adopting the other strategies. From Schwartz and Zamboanga's point of view, the individuals who adopt or retain a single culture (the assimilation and separation strategies) would have less stress than the bicultural individuals who manage two different cultures simultaneously. In addition, Berry's acculturative stress model indicates the tendency of declining acculturative stress with time yet, Chen (2011) points out the possibility of increasing health problems with duration; in his view, individuals who experience acculturative stress longer would have more health problems.

While most immigrants undergo cultural transition during the process of acculturation, changes in health and health behaviours also occur as a part of the acculturation (Berry, 2003; Sam \& Berry, 2010). Despite numerous acculturation studies, the interrelationship between health and acculturation, more specifically, the heterogeneity of immigrants' health and health outcomes regarding their degrees of acculturation and duration of stay does not provide clarity.

\footnotetext{
${ }^{16}$ See the Section 2.4 Acculturation (p. 30).
} 


\subsection{Immigrants' health service utilisation}

In addition to the increased attention regarding the health of immigrants and its relationship to acculturation, some researchers have conducted studies to establish if there is a correlation between immigrants' health service utilisation and acculturation. These researchers found that immigrants' health service utilisation rose with the duration of residence and with the degree of acculturation (Akresh, 2009; Fassaert et al., 2009; Kim, Han, Kim, \& Duong, 2002; Salant \& Lauderdale, 2003). Immigrants' health is generally determined by the utilisation of appropriate and effective healthcare (accessibility, availability, and affordability) and quality of health services in the host country (Davies et al., 2009). Although obtaining adequate and timely healthcare is considered a prerequisite for one's health, immigrants' health services access and utilisation is often limited by their disadvantaged immigrant status: socioeconomic and legal status, cultural differences, language difficulties, unfamiliarity of host healthcare system, and insufficient knowledge and information regarding entitlements to care and availability of services (Davies et al., 2009; Galanis et al., 2013). In this regard, immigrants' health service utilisation is a result of interaction between health needs, socio-economic and cultural factors, and health resources, rather than quality of health services in the host country alone (Stephenson \& Hennink, 2004; Uchudi, 2001).

The measurement of health service utilisation is a useful way to monitor health inequalities between immigrants and native-born citizens; groups with high healthcare needs would appear with a higher usage of health service than other groups (Crampton, Jatrana, Lay-Yee, \& Davis, 2007). As international studies on immigrants' health have shown, predominant disparities in healthcare between immigrant groups and the native-born population are presented; the health service utilisation of immigrants is markedly lower than that of the non-migrant population (Anderson, 2008; Carrasquillo, Carrasquillo, \& Shea, 2000; de Luca et al., 2013; Derose, Bahney, Lurie, \& Escarce, 2009; Derose et al., 2007). This disparity was also present in New Zealand. The New Zealand Health Survey 2011/12 (Ministry of Health, 2012b) found a lower use of primary health services amongst Asian ethnic groups such as Korean immigrants. According to the survey, Asian immigrants have 
comparatively low levels of health-related risk factors including smoking, and have less incidence of some chronic conditions, for example, asthma, arthritis and chronic pain. Thus, Asian's lower health service utilisation compared to other ethnic groups is partly due to their reduced needs of healthcare as a consequence of their overall better health status otherwise known as the healthy immigrant effect (Kennedy et al., 2006; McDonald \& Kennedy, 2004; Mehta, 2012).

In contrast, there is an argument that the lower health service utilisation including postponed seeking of healthcare of ethnic minority groups is due to barriers, such as language difficulties, unfamiliarity of the healthcare system, low income, cultural incompetency, and discrimination (Derose et al., 2009; Derose et al., 2007; Leclere et al., 1994; Shin, Song, Kim, \& Probst, 2005). An ethnographic study examining health service utilisation of immigrants (Indian, Korean, and Chinese) in New Zealand (Anderson, 2008) found that the factors affecting health service utilisation of these ethnic groups are mainly due to the knowledge and experiences of health services in both the host and home countries, language skills, and cultural competency. For example, the general practitioner (GP) and referral system of New Zealand, which are different from the healthcare systems of the participants' home countries, influence their health service utilisation. Similarly, a study on Korean immigrants' health-seeking behaviours in Hawaii (Choi, 2013a) claims that Koreans' under-utilisation of the health service is a result of their low coverage of health insurance, the high medical costs, and lack of information of the health service system in Hawaii.

Studies on immigrants' health have found that immigrants' under-utilisation of health services, regardless of its reasons, declines along with their duration of residence (Akresh, 2009; Fassaert et al., 2009; Leclere et al., 1994). Individuals with longer duration in a country become more comfortable with the use of the host language and gain familiarity with the healthcare system, resulting in increased health service utilisation (Akresh, 2009). It is also argued that this increased utilisation occurs due to the development of poorer health as a consequence of the length of stay (Clough, Lee, \& Chae, 2013). For instance, as immigrants adopt the dominant culture, especially the unhealthy lifestyle including drinking and eating 
habits, the number of obese Asian immigrants in New Zealand is rapidly increasing (Scragg, 2010). The health gap between immigrants and non-immigrants diminishes gradually as the duration increases, which can be explained by the healthy immigrant effect ${ }^{17}$ described above (Fennelly, 2007; Ministry of Health, 2006). Ku and Matani (2001) set out to understand immigrants' access to health service related to their insurance status in the USA and found that noncitizen adults had reduced health service utilisation while naturalised citizens were patterned similar to that of the native-born citizens.

In summary, immigrants' health service utilisation increases along with time in the host country, and the degree of acculturation is positively associated with their health service utilisation. However, immigrants' health service under-utilisation, whether caused by their better health status or barriers, is not well understood. It is also not clear if the increased health service utilisation of immigrants, in time, is due to immigrants' imitating the unhealthy lifestyles of the native-born population or because of improved language skills and understanding of the healthcare system in the host countries.

\subsection{Health-seeking behaviour of immigrants}

"Health-seeking behaviour is an activity motivated by a desire to protect health by avoiding illness or a desire to increase one's level of health in either the absence or presence of illness" (Pender, Murdaugh, \& Parsons, 2002, p. 34). Studies on health-seeking behaviour focus on individuals' illness behaviour and aim to understand the factors influencing their health beliefs and decisions to seek healthcare (Grundy \& Annear, 2010; MacKian, 2003). This behaviour is shaped by individuals' sequential healthcare activities based on their perceptions and beliefs with respect to health and healthcare; the ways of observing, interpreting and responding to their health symptoms are influenced by their personal evaluation of these activities (Anwar et al., 2012). MacKian (2003) claims that individuals' healthseeking behaviour is associated with physical, social, and cultural factors; and it reflects an inter-relationship between the individuals and society. To understand any

\footnotetext{
${ }^{17}$ See the Section 2.6 Immigrants' health and acculturation (p. 38).
} 
specific health needs and issues of individuals and groups, prior knowledge of health-seeking behaviour, including the factors affecting this behaviour, is necessary (Hausmann-Muela, Muela Ribera, \& Nyamongo, 2003).

While immigrants encounter a new culture and new environment in the host country, their health-seeking behaviour is challenged by the unfamiliar host health services (Choi, 2013b). As a consequence of interaction with the host health services, immigrants' health-seeking behaviour is modified and reconstructed by the healthcare they experienced; in other words, how immigrants perceive the health services they received in the host country is reflected in their health-seeking behaviour (Choi, 2013a; Leduc \& Proulx, 2004). Accordingly, the factors determining their health-seeking behaviour are the experiences and perceptions of the host health services, immigrant status (such as socio-economic and legal status, and duration of residence, language proficiency), health beliefs, illness perceptions, and the social contexts of the host and home countries (Choi, 2009, 2013b; Leduc \& Proulx, 2004; Portes, Kyle, \& Eaton, 1992). This means, immigrants' health-seeking behaviour varies not only between immigrant and non-immigrant groups, but also amongst immigrants (Choi, 2013b; Ell \& Castaneda, 1998).

Immigrants' healthcare seeking behaviours commonly fall into two pathways; utilising formal services from the host healthcare system and looking for alternative services (Gideon, 2011; Grundy \& Annear, 2010). Immigrants generally look for alternatives in order to solve their health problems when they encounter obstacles to gain access to the formal health services (the host healthcare system) because of their disadvantaged immigrant status and/or when they want to avoid the formal health services due to their healthcare preferences. Many of them initially tend to selfmedicate, using herbal or traditional folk remedies, traditional medicines, and illegal private practices as alternative strategies to replace the formal healthcare services. They employ these alternatives before and/or after utilising formal care, or with formal care simultaneously (Gideon, 2011; Kim et al., 2002). One of the major issues facing immigrants is negotiating the health service system of the host country (Choi, 2013a; Leduc \& Proulx, 2004; Lee, Kearns, et al., 2010). 


\subsection{Korean immigrants' health-seeking behaviour in New Zealand}

Recent studies on Korean immigrants' health-seeking behaviour in the USA and New Zealand report that immigrated Koreans return to their homeland for healthcare (Choi, 2013a; Lee, Kearns, et al., 2010; Son, 2013). This specific healthseeking behaviour is a growing phenomenon amongst immigrant populations (particularly amongst recent immigrants), and it is often referred to as "medical return" (Horton \& Cole, 2011, p. 1). However, why certain immigrants choose medical return instead of formal services in the host country, in spite of health risks (long waiting period to get healthcare) and cost and time to travel is unclear (Horton $\&$ Cole, 2011). Choi (2013a) carried out a qualitative research with 20 Koreans in Hawaii to understand their health-seeking behaviours and found that the Korean immigrants considered returning to their home country as an alternative choice for healthcare. This health-seeking behaviour of Koreans was determined by a lack of health insurance and high medical costs, unfamiliarity with the healthcare system, and the cultural and linguistic comfort of health services in their country of origin. The Korean participants considered a trip to Korea for healthcare a rational choice (Choi, 2013a).

Mexican immigrants in California are another group known for medical return (Horton \& Cole, 2011). They often cross the Mexican border to access healthcare in Mexico, and the reasons for this behaviour is similar to that of Koreans in Hawaii; high medical costs, a lack of insurance, and type of medical practice which they prefer. Mexicans in Horton and Cole's study who lived near the Mexican border also chose "convenience" as one of the reasons for their medical return. In addition, the UK large-scale survey of over 1,000 Latin Americans in London found that approximately $30 \%$ of them seek healthcare in Latin America, and their reasons for medical return were largely their immigrant legal status and unfamiliarity of the host healthcare system (Mcllwaine, Cock, \& Linneker, 2011). Latin-Americans who looked for healthcare in their home country were largely undocumented or recent immigrants to the UK.

Some Korean immigrants in New Zealand also choose Korea for medical care. According to Lee, Kearns, et al. (2010), first-generation Korean immigrants 
who are permanent residents or citizens of New Zealand (most with more than 10 years residency) place more importance on the "affective" aspects of healthcare than the available "effective" medical care (p. 114). Seeking affective care was their main reason for medical return to Korea beyond the barriers of healthcare such as high medical costs, a lack of health insurance, and incompetency in the New Zealand healthcare system. In the New Zealand healthcare system (which is a largely publicly funded system), the secondary and tertiary services in public hospitals are free in both hospital and outpatient treatment including 24-hour accident and emergency clinics; primary healthcare practices (such as visits to GPs and dentists) require partial or full payment (Cumming et al., 2014). Immigrants in New Zealand with permanent residence or citizenship status are entitled to the same healthcare benefits as native-born citizens.

It has been reported that the majority of Korean immigrants in New Zealand are generally wealthier and well educated, and they came to New Zealand for a better quality of life rather than for economic reasons (Yoon \& Yim, 1997). The specific health-seeking behaviour of Korean immigrants in New Zealand seeking healthcare in their homeland cannot be explained by that of Korean and Mexican immigrants in the USA (a lack of health insurance, high medical costs, and incompetency in the host healthcare system), or Latin Americans in the UK (immigrant legal status and unfamiliarity with the host healthcare system). As shown in the literature above, most immigrants tend to assimilate into the healthcare system in the host country along with duration of residence (Akresh, 2009; Choi, 2013a; Ku \& Matani, 2001). Likewise, naturalized citizens have a tendency to adopt the health-seeking behaviour of the native-born citizens ( $\mathrm{Ku} \& \mathrm{Matani}, 2001)$. However, the first generation Koreans who have lived more than 10 years in New Zealand and who have access to free public healthcare still seek healthcare in their home country despite putting their health at risk by delaying care (Lee, Kearns, et al., 2010). This health-seeking behaviour of Korean immigrants (medical return) can be viewed as a failure to negotiate the healthcare system in New Zealand (Elliott \& Gillie, 1998; Ormond, 2014). Although an increasing interest in the health of immigrants in New Zealand has been recognised there is insufficient literature to explain Koreans' health or health-seeking behaviour. 


\subsection{Illness perception and cultural implications}

Individuals' health and health-seeking behaviours are considerably influenced by their perceptions of health and illnesses; how they perceive their health and illnesses affect their responses to illness symptoms and healthcare, and outcomes of illness (Boruchovitch \& Mednick, 2002; Perez, 2015). While disease is referred to as a bio-medical condition (a clinical concept), illness is a culturally and socially constructed health condition (a subjective concept) (Eisenberg, 1977; Prior, Chun, \& Huat, 2000). Individuals perceive and interpret their illness symptoms and conditions differently (even with a similar illness), and it is generally due to their different illness perceptions, which are formed based on personal beliefs, knowledge, and illness experiences (Conrad \& Barker, 2010; Petrie \& Weinman, 2012). In the social constructivists' view, perception is socially constructed through the process of interaction within a social context, and not a discovered concept (Conrad \& Barker, 2010). How society responds to a certain illness influences how individuals in that society define the illness; therefore, the socially constructed illness perceptions are not be the same amongst different groups (Mishra, Kusuma, \& Babu, 2013; Salant \& Lauderdale, 2003). According to Mishra et al., people who are under the same cultural influence share their own meanings of health and illnesses. "Culture constitutes a shared system of meaning, the way that people experience, perceive, and interpret their world" (Becker, Gates, \& Newsom, 2004, p. 2066).

Immigrants who come from different cultural backgrounds and have different healthcare experiences, exhibit different perceptions of illnesses and expectations for healthcare (Choi, 2013b; Mead \& Roland, 2009). Immigrants perceive illnesses and respond to healthcare based on their experiences and through their own cultural lens (Mishra et al., 2013; Salant \& Lauderdale, 2003). For example, "Hwa-byung" is one of the cultural syndromes or culture bound syndromes ${ }^{18}$ only found in Koreans and Korean immigrants (Choi \& Yeom, 2011; Lee, 2014). Hwa-byung is a psychiatric illness caused by chronic distress and it is literally an anger illness ("Hwa" means anger and "Byung" means illness that is also known as anxiety disorder or major

\footnotetext{
${ }^{18}$ Culture bound syndromes are generally considered as indigenous illnesses found in specific cultural groups and communities (American Psychiatric Association, 2005).
} 
depressive disorder) (Lee, 2015). In Korean culture, people are encouraged not to express their emotional feelings (particularly negative feelings such as anger, sorrow, and disappointment) and encouraged to avoid confrontation in order to preserve harmony within the hierarchical relationships (Ninnemann, 2012). While people suppress their emotions and feelings to keep harmony in their families and personal relationships, they experience chronic distress and anger caused by their accumulated emotional repressions; consequently, over time Hwa-byung is developed by this emotional disruption (Choi \& Yeom, 2011; Ninnemann, 2012). In the traditionally patriarchal society of Korea, Hwa-byung has been reported as present in 5-13\% of women in their 40s-60s (Lee, Wachholtz, \& Choi, 2014). This illness is generally accompanied by both somatic and psychological symptoms such as heart palpitation, chest tightness, headache, indigestion, panic, insomnia, anxiety, anger, and depression. Although patients are aware of the causes of their Hwa-byung which are related to psychological problems and issues, they are less likely to seek help from psychologists or psychiatrists (Lee, Wachholtz, et al., 2014; Park, 2014). Rather they focus on medical treatment for only physical symptoms and it is because of the social perception towards mental illnesses in Korean culture; mental illnesses are often stigmatised and seen as a source of shame (Kim \& Rew, 1994; Park, 2014).

This illness was also detected amongst the middle aged or older Korean American women in the USA (Kim \& Rew, 1994; Lee, Wachholtz, et al., 2014; Lee, 2014), and elderly Korean immigrants both in males and females in New Zealand (Park, 2014). According to Park, Hwa-byung was one of the major illness experienced by elderly first-generation Korean New Zealanders even though the causes of the developing Hwa-byung are different to that of Koreans in Korea (e.g., language difficulties, adjustment problems, cultural and social isolation, and elder mistreatment in the family). Similar symptoms of Hwa-byung are reported by the participants; for example, chest pain, heart related discomforts, and a feeling of tightness and heaviness in chest. However, there was no heart related illness diagnosed by their doctors in New Zealand. It would be very difficult to expect a correct diagnosis and a proper medical treatment for this culture bound syndrome Hwa-byung from doctors who do not understand its causes and symptoms, and who are not aware of its cultural context. 
As individuals' illness perceptions and responses are heavily affected by cultural and social factors, the concepts and perceptions of illness change when the cultural and social norms are changed (Jumah \& Duda, 2008; Mishra et al., 2013). The traditional and cultural illness perceptions in non-Western societies are challenged and replaced by Western concepts as a result of globalization (Frederick, Forbes, \& Anna, 2008). Jumah and Duda (2008) tested Ghanaian males' perception of ideal woman's body images, and found that Ghanaian males' traditional preferences for female's body image have changed; from heavy and large body types to average or slim types. Likewise, Ghanaian women's perception toward their own body image has also changed from overweight to slim or average, even though most female participants in the study believed that Ghanaian men are still more attracted to heavier female body types.

In a culturally diverse society, immigrants experience changes in health concept and illness perception during the process of acculturation (Mishra et al., 2013; Salant \& Lauderdale, 2003). "Illness is experiences", and when immigrants experience illnesses and healthcare in the host societies they learn different meanings of illnesses from others, and they compare their healthcare experiences with the previous ones in their home countries (Jennings, 1986, p. 866). As a result, the changes in illness perception take place as a part of acculturation. The raised concern is that immigrants' illness perception relating to healthcare creates difficulties in making an illness diagnosis (Conrad \& Barker, 2010). Although individuals claim symptoms represent an illness, these symptoms sometimes do not reveal physical abnormalities, that are then acknowledged by physicians in order to provide medical treatment. Immigrants' illnesses, which are often deeply entwined with various cultural meanings and influences, can be more difficult for physicians in the host countries to acknowledge. Without any medically recognised symptom no medical treatment is offered, rather the claim of an illness raises some suspicions for host countries' medical professionals (Padilla \& Villalobos, 2007). Consequently, immigrants' different understandings of illnesses and different healthcare expectations, which are rooted in their cultural perception, are often considered as a barrier to healthcare in the host societies 


\subsection{Patient satisfaction}

Patients' perceptions of their healthcare are considered as key determinants for the quality of care (Suurmond, Uiters, de Bruijne, Stronks, \& Essink-Bot, 2011; Weingart et al., 2005). How patients perceive the health service provided is directly related to patient satisfaction, and their levels of satisfaction are manifested as the quality of the service they received (Hudson, Zarifeh, Young, \& Wells, 2012). If the quality of the health service is a prerequisite to elicit the patient satisfaction (Naidu, 2009), the quality of the healthcare system could be measured by the degrees of patient satisfaction (Sajid \& Baig, 2007; Senić \& Marinković, 2013).

The interaction between patient and physician, and their trust relationship has been emphasised as a major contributor to patient satisfaction with positive outcomes (Alrashdi, 2012; Harmsen, Bernsen, Bruijnzeels, \& Meeuwesen, 2008; Hudson et al., 2012; Suurmond et al., 2011). Patients who are satisfied with their healthcare tend to comply with physician's treatment guidance including follow-ups; resulting in better health outcomes, faster recovery, and reduced medical cost (Alrashdi, 2012; Choi, Kim, Lee, Lee, \& Cho, 2004; Hudson et al., 2012; Sixma, Kerssens, Campen, \& Peters, 1998). It is also noteworthy that the understanding gap between patients and physicians has been identified as one of the main causes of patients' dissatisfaction (Alrashdi, 2012; Harmsen et al., 2008; Hudson et al., 2012; Suurmond et al., 2011). Immigrants' unsatisfactory healthcare experiences have been found to be mainly caused by communication problems such as miscommunication and misunderstanding (Harmsen et al., 2008; Hudson et al., 2012; Suurmond et al., 2011). Ethnic minority immigrants are less likely to be satisfied with the health service in their host countries, and it is largely due to insufficient understanding of their physicians as to their healthcare (Harmsen et al., 2008). For example, Harmsen et al. (2008) in the Netherlands examined immigrants' levels of healthcare satisfaction and their perceived service quality with respect to cultural differences and language proficiency, and found that immigrants were less likely to be satisfied with their healthcare than the non-immigrant population. Immigrants' negative perceptions of their healthcare were directly related to the communication problems (insufficient understanding between immigrant patients and their healthcare 
providers), and this was a major cause of their low satisfaction. Immigrants with negative healthcare experiences reported that they were not well informed when receiving healthcare; as a result, they felt they were being ignored (Suurmond et al., 2011). Lack of mutual understanding and communication difficulties contributed to immigrants' negative healthcare experiences and also mistrust between immigrants and their physicians.

The role of the patient-physician interaction and physician's attitudes have been acknowledged as a major factor affecting patient satisfaction; immigrants, who had communication problems with their physicians, show lower levels of satisfaction with their healthcare (Harmsen et al., 2008; Migge \& Gilmartin, 2011; Salant \& Lauderdale, 2003; Suurmond et al., 2011). Schouten and Meeuwesen's (2006) review of the literature related to the communication between patients and physicians identified five variables contributing to immigrants' communication problems: different beliefs as to health and illness; different cultural values; different perceptions on doctor-patient relationship; racism; and language barriers. They concluded that culture and ethnicity are barriers (together with their language barriers) to building an affirmative patient-physician relationship. For example, Asian immigrants, familiar with the hierarchical doctor-patient relationship, have been found to generally respect their doctors as a primary decision maker and often complain about their doctors in the host countries who consider patients the main actor in the decision-making process (Clough et al., 2013; Harmsen et al., 2008; Tam Ashing, Padilla, Tejero, \& Kagawa-Singer, 2003). They are more likely to have followed their doctors' detailed instructions as to the treatments such as diet and daily life activities (Tam Ashing et al., 2003).

The gap between the two healthcare systems of the host and home countries is also indicated as a determinant in immigrants' satisfaction with their healthcare; immigrants, who came from countries with various healthcare systems, perceive the host health services differently depending on their previous healthcare experiences in their home countries (Lien, Nafstad, \& Rosvold, 2008; Mead \& Roland, 2009; O'Donnell, Higgins, Chauhan, \& Mullen, 2008). Immigrants were largely dissatisfied with healthcare from their GPs if they had never previously experienced the GP 
service, and they viewed the GPs as less qualified doctors than the specialised doctors (O'Donnell, Higgins, Chauhan, \& Mullen, 2007; 2008). As a result, they tended to withdraw from healthcare with the GP service, preferring to see specialists. It would appear that immigrants evaluate their healthcare in the host countries based on their healthcare expectations; what immigrants expect in healthcare depends on what type of healthcare they experienced in their home countries (Bowling et al., 2012; Bowling, Rowe, \& McKee, 2013; Hudson et al., 2012). Immigrants' healthcare adaptation to the host system is influenced by the type of healthcare system and the level of health services in their home countries (Lipson, Weinstein, Gladstone, \& Sarnoff, 2003). If the gap between the home and host countries' healthcare systems is great, adapting would be more difficult.

While the abundant empirical literature has revealed the positive association between patient satisfaction and health outcomes (Alrashdi, 2012; Hudson et al., 2012; Sajid \& Baig, 2007; Senić \& Marinković, 2013; Tucker \& Adams, 2001), achieving a high level of patients satisfaction has also been emphasised as a source of profit within the competitive medical environment (Choi et al., 2004; Tucker \& Adams, 2001; Zeithaml, 2000). However, patients' assessments of health service quality, as measured by their levels of satisfaction, have been questioned for their validity (Alrashdi, 2012; Choi et al., 2004; Chow, Mayer, Darzi, \& Athanasiou, 2009; Godil et al., 2013; Hudson et al., 2012; Naidu, 2009; Peck et al., 2001). Consumer satisfaction is often described as a feeling or an affective/cognitive response towards evaluation of a received service (Hu, Kandampully, \& Juwaheer, 2009; Senić \& Marinković, 2013; Westbrook, 1981); likewise, patient satisfaction is a perception which is heavily dependent on their subjective evaluation of the experienced service (Senić \& Marinković, 2013). Patients' evaluations are largely based on the functional quality of the service rather than its technical quality (Alrashdi, 2012; Senić \& Marinković, 2013). How the service is provided (functional quality) is known to have more impacts on patients' evaluation rather than the medical care (technical quality) itself, and it is largely because of patients' lack of knowledge and expertise to assess the technical quality of the service (Alrashdi, 2012). For example, according to Godil et al. (2013), patient satisfaction is not a valid measure for the quality of healthcare. Godil et al. conducted research with 422 
spine surgical patients and found that patient satisfaction was not associated with surgical morbidity and healthcare quality. These findings indicate that patient's views on the quality of the service may differ to that of physicians who provide the service (Suurmond et al., 2011).

Shi and Stevens (2005), who examined the association between race/ethnicity and socioeconomic status with respect to healthcare in the USA, found that whites (non-Hispanic) had higher levels of expectations and lower levels of satisfaction toward the USA healthcare system. Compared to whites, all other ethnic minority groups in the study showed relatively lower levels of expectations with higher levels of satisfaction. According to the researchers, the lowered health service expectations of minority groups were a result of the difficulties in health service utilisation, or their negative healthcare experiences in the host country. They were less likely to express their unmet needs because of their lowered expectations; this means that their higher levels of satisfaction compared to white counterparts were not influenced by the service quality.

Health service quality is positively associated with patient satisfaction (Naidu, 2009; Tucker \& Adams, 2001). According to Petersen (1988), "it really does not matter if the patient is right or wrong. What counts is how the patient felt even though the caregiver's perception of reality may be quite different" (p. 25). Godil et al. (2013) argue that if healthcare is ineffective and/or unsafe, the service quality would not be accepted even if the patients are satisfied with the service. They assert that patient satisfaction alone does not guarantee the quality of service.

\subsection{Quality of health service}

Although service satisfaction as a proxy for the service quality has raised debates, patient satisfaction is a valuable source of health service quality assessment (Alrashdi, 2012; Choi et al., 2004). Service quality is often defined as a gap between expected service and actual service received; perceived quality is a consequence of a comparison between expected and received services (Parasuraman, Zeithaml, \& Berry, 1985). Health service quality can be assessed by patients' evaluation, as they are the ones who experience the entire process of the service and its outcomes 
(Alrashdi, 2012). Although there have always been expectations for quality in health service and constant interests in its improvements, it is not easy to assess quality due to its elusiveness and heterogeneity characteristics (Mohammad \& Alhamadani, 2011). Unlike the other tangible products (e.g., vehicles, gadgets, or clothing), healthcare is a process of service including various interactions between patients and health service providers (Jun, Peterson, \& Zsidisin, 1998; Mitchell, 2008; Mosadeghrad, 2013). The health services provided are less likely to have the constant levels of quality; likewise, the perceived quality would be interpreted differently from person to person (Jun et al., 1998; Mosadeghrad, 2013) and it is difficult to confirm the quality before the service has been delivered to the patients (Mosadeghrad, 2013). Above all, the evaluation of the quality of health services is complex because it involves highly specialised expertise, multi-dimensional interactions, and performance risks (Alrashdi, 2012; Rashid \& Jusoff, 2009).

Health service quality is commonly divided into two distinct dimensions: technical service quality and functional service quality (Fiala, 2012; Grönroos, 1984; Jun et al., 1998; Papanikolaou \& Zygiaris, 2014; Parasuraman et al., 1985). While technical quality is related to the core medical service; that is, technical accuracy, procedures, and its outcomes (what is achieved), functional quality is about the manner of service delivery (how it is achieved). Donabedian (1988) categorised health services into three different domains for the quality assessment: structure, process, and outcomes. Structure indicates the quality of healthcare system (e.g., facilities, technologies, and medical staff), process is related to healthcare providers' activities (e.g., diagnosis and treatments), and outcome refers to the improvements in health as a result of healthcare. He claims that a good health service structure can increase the quality of the service processes, and that good service processes result in good healthcare outcomes. In order to improve the service quality, the assessments should take into account different perspectives of different stakeholders as the key contributors, such as the perspectives from patients, health service providers, and communities (Donabedian, 1988; Jun et al., 1998; Mosadeghrad, 2013). 


\subsection{Patients rather than consumers}

The traditional patient-physician relationship is predominantly physiciancentred whereby the role of the physician is highly emphasised with autonomy (Alexander, Hearld, Mittler, \& Harvey, 2012; Alrashdi, 2012; Berger, Braehler, \& Ernst, 2012; Kaba \& Sooriakumaran, 2007). In the physician-centred setting, physicians actively make decisions on their own, and their patients passively comply with the decisions (Kaba \& Sooriakumaran, 2007). In recent years, the autonomy of physicians has shifted to their patients (Berger et al., 2012; Kaba \& Sooriakumaran, 2007; Levinson, Kao, Kuby, \& Thisted, 2005). Unlike the passive patients in the physician dominant relationship, todays' patients seek treatment information; express their preferences; discuss their healthcare options; and participate in the healthcare decision-making. Patients, who are actively involved in their healthcare, are usually more interested in and satisfied with their healthcare, and they are more likely to carry out their treatment effectively and adopt healthy behaviours; consequently, they have better health outcomes (Alexander et al., 2012; Eaton, Roberts, \& Turner, 2015; Kuehn, 2009; Price, Bereknyei, Kuby, Levinson, \& Braddock, 2012). In the UK, almost $90 \%$ of GPs implement shared decision-making during the medical interaction with their patients (Blundell et al., 2011).

Patient-oriented care is now endorsed as a key principle in the healthcare setting, and the role of the patient in the health service has been empowered more than ever (Berger et al., 2012; Deber, Kraetschmer, Urowitz, \& Sharpe, 2005; Levinson et al., 2005). It is furthermore questioned whether the term "patient" still applies due to their empowerment and autonomy (Deber et al., 2005). The original meanings of patient includes "patience; sufferance" (from Old French pacience), "patience, endurance, submission; quality of suffering" (from Latin patientia) (Zaner, 2015). In contrast, today's patients, who articulate their demands, can alter their treatment and influence the relationship with their physicians may not be properly explained by the concept of the term "patient", which is largely dependent and powerless (Mossma, 2014). These changed roles and attitudes of patients in modern 
medicine render them a new identity; "consumers" 19 or "customers" 20 who acquire or buy goods and services (Brown, 1993; Deber et al., 2005; McLaughlin, 2009;

Mossma, 2014). The benefits of consumerism in healthcare were emphasised:

Consumerism-a societal movement grounded in education and transparency of information-enables and engages individuals more directly in selection and purchasing decisions.... The principles of consumerism involve higher quality, wider variety, better access, innovation, and lower costs. These principles allow consumers to flex their market power and drive efficiency into the system. (Blank, 2010, p. 9)

Acknowledging patients as consumers helps physicians to be aware of patient equality and rights in the healthcare relationship, and encourages physicians to know their patients better and to meet their needs (Brown, 1993). Once patients were considered as consumers who were buyers of the health services (i.e., consumers or customers), physicians were seen as sellers who provide the services (Mossma, 2014). Subsequently, the physician-patient relationship was replaced by the relationship of provider-consumer which is governed by the supply-demand principle (Deber, 2000; Mossma, 2014; Naidu, 2009). Different labels describe different identities, characteristics of individuals, and their relationships (McLaughlin, 2009; Mossma, 2014; Naidu, 2009). Conversely, once people are given labels, they are influenced by their labels, and tend to act accordingly.

While healthcare autonomy of patients is recognised as the consumers' right and their choices in healthcare emphasised as consumers' power in modern medicine (Deber et al., 2005), this consumerism in healthcare has raised scepticism (McLaughlin, 2009; Mold, 2015; Mossma, 2014). In general, consumers/customers are assumed as rational individuals who are able to make the right choices in their healthcare; health service consumers thoughtfully consider variables and consequences of their healthcare in order to meet their needs and expectations

\footnotetext{
19 "Consumer" from the Latin (consumere) is defined as "to take completely, to take up. One who acquires goods or services; a buyer" (Brown, 1993, p. 18). Patients were first called consumers by medical economists in the USA in the 1930s (Mold, 2015).

20 "Customer" from the Middle English (word for tax collector) refers to a person who purchases products or services (Deber et al., 2005).
} 
(McLaughlin, 2009). However, what would be a right thing to do by the healthcare providers, if their patients' choices/decisions are inappropriate (McLaughlin, 2009; Mold, 2015)? Shortus, Kemp, McKenzie, and Harris (2013) conducted a study focused on healthcare providers' perspectives regarding patients' involvement in medical decision-making and found that healthcare providers often experienced a conflict between two different responsibilities; one was achieving ideal health outcomes (evidence-based medicine) for their patients, and the other was respecting their patient's decisions. The healthcare providers all acknowledged achieving "the best possible outcomes in the best possible way" (p. 193) for their patients as a main goal. The providers, who emphasised the importance of achieving ideal outcomes rather than respecting their patients' decisions, believed that "a good (and professionally responsible) provider was one with the ability and desire to try to change a patient's priorities and preferences, rather than one who simply respected their priorities when they differed and did not try to change them" (Shortus et al., 2013, p. 193).

The appropriateness of the notion of consumers in health service has been challenged and criticised as seeing healthcare as shopping (Mold, 2015). It has been noted that most patients want to be fully engaged in their healthcare decision-making (Deber et al., 2005; Kraetschmer, Sharpe, Urowitz, \& Deber, 2004; Levinson et al., 2005); however, many still like to rely on their doctor's opinion for their treatment (Levinson et al., 2005; Price et al., 2012). For example, Levinson et al.’s (2005) study on public preferences for participating in decision-making in the USA found that while most (96\%) of the 2,765 respondents wished to be involved in their healthcare (i.e., discuss treatment options and share opinions with their doctors), approximately half (52\%) wanted their physicians to make the final decisions on their behalf, and $44 \%$ were in favour of a physician-directed approach in their relationship with physicians.

The modern patients, who expect to be more autonomous as consumers, still want to be called a "patient" rather than "consumer" or "customer" (Anczewska et al., 2011; Deber et al., 2005; Mossma, 2014). Deber et al. (2005) surveyed the feelings and preferences of patients on their labels (how they want to be called) and 
found that the patients were not comfortable with the terms client, customer, or consumer, but were comfortable with the term patient. Respondents in this study displayed different attitudes compared to traditional patients; they often complained about the long wait for healthcare and highly desired to be engaged in healthcare decision-making with their physicians rather than passively follow their doctors' decisions. However, they were in favour of the patient-physician relationship over the consumer-provider relationship. Krugman (2011, April 20) argues against viewing patients as consumers and perceiving healthcare as merely financial transactions. He emphasises that medical decisions are life and death decisions, which require specialised expertise and heavy responsibilities; and insists that health services should not be considered as ordinary services, and doctors should not be thought of as people who sell their services to consumers. While healthcare is acknowledged as a patient right (Maruthappu, Ologunde, \& Gunarajasingam, 2013), the question raised is whether consumers deserve this right (Wen, 2013).

\subsection{Person-centred care}

In general, physicians are expected to demonstrate a certain level of professionalism within the relationship with their patients (Robbins \& Galperin, 2010). Physicians have professional obligations (Cruess, Cruess, \& Johnston, 2004), and these obligations distinguish them from other professionals (Martimianakis, Maniate, \& Hodges, 2009). Emanuel (1988) identified the professional role of physicians, "the objective of the medical profession is devotion to a moral ideal-in particular healing the sick and rendering the ill healthy and well” (p. 1686). Physicians, who take an oath to commit to their patients and society are assumed to act in the best interests of their patients, rather than their own interests (Minkoff \& Ecker, 2015; Pellegrino, 2002). Based on this assumption, patients and society put their faith in physicians, and the therapeutic relationship between patient-physician is constructed based on this faith (Minkoff \& Ecker, 2015).

Berwick, a medical doctor (paediatrician), described his fear of becoming a patient. "It is the loss of influence on what happens to me. It is the image of myself in a hospital gown, homogenized, anonymous, powerless, no longer myself”' (2009, 
p. 564). His fear was mainly due to a loss of self rather than medical errors or unreliability, and the fear was from his understanding that physicians follow their own needs and desires rather than those of the patients. However, as demonstrated by the emotions expressed by Berwick, a patient is not an object but a person with his/her own feelings and perceptions (Rogers \& Koch, 1959). They have knowledge about their illnesses based on their own experiences that their physicians may not know. The notion of person-centeredness was developed by the American psychologist Carl Rogers in the early 1940s (Leplege et al., 2007; Morgan \& Yoder, 2012). There are a number of terms referring to the concept person-centred, and they are often used interchangeably; for example, patient-centred, people centred, and relationship-centred (McCormack, Karlsson, Dewing, \& Lerdal, 2010). Personcentred care, as a holistic approach, considers one as a whole person beyond a patient, and respects one's values and beliefs (Leplege et al., 2007; McCormack et al., 2010). It emphasises a trust relationship between patient and physician in order to provide healthcare based on the patient's point of view, rather than the views of physicians or the healthcare system (Neuwelt \& Matheson, 2012; Pelzang, 2010). However, Omonzejele (2005) argues that respecting patients and the wishes of patients are two different issues. In Berwick's (2009) view, patients' wishes and needs are something that physicians need to know and learn. When the wishes of patients are not desirable according to the physicians' view, physicians should reinform the patients of the relevant information and the technical evidence. If a number of patients made the same but wrong choices (e.g., requesting needless Magnetic Resonance Imaging [MRI]), then it would be a sign of failure in education and communication between patient-physician, which would need to be improved.

While patients and society put their faith in physicians and expect them to provide altruistic services based on morality and integrity (Cruess \& Cruess, 2008; Pellegrino, 2002), physicians are expected to keep an emotional distance from their patients as a protocol in hospital settings (Robbins \& Galperin, 2010). Individuals who have low levels of empathy are less likely to be motivated by altruism (Batson, 2002). Physicians who are highly empathetic may experience distress if they are unable to help their patients, and it could result in avoiding feelings of empathy in order to stay away from altruistic engagement with their patients (Burks, Youll, \& 
Durtschi, 2012). This empathy avoidance could lead to less effective healthcare with dissatisfactory healthcare experiences for both the patients as healthcare recipients and the physicians as providers (Batson, 2002; Burks et al., 2012). Palmer (2007) criticises the medical environment which teaches and encourages professionals to suppress their emotions as a strategy to survive as physicians. "Don't wear your heart on your sleeve" (p. 9), that is, hide your emotions to stay safe. Palmer articulates the importance of the ability to understand emotions of others and themselves in healthcare which refers to emotional intelligence ${ }^{21}$. Patients who experience caring, empathic, reliable and responsive care (person-centred care) demonstrate not only increased satisfaction but also improved healthcare outcomes, engaging in healthy behaviours (Hibbard \& Greene, 2013; Rogers, 1979; Turney \& Kao, 2012).

Although person-centredness or patient-centredness in health services has become a major indicator in quality of care (Epstein \& Street, 2011); the meaning of patient-centredness changes depending on different interests (Berwick, 2009). In the consumer-oriented approach, patient-centredness proposes "let us know what you need and want, and that is what we will offer" (Berwick, 2009, p. w558), which is based on the assumption that patients are always right. Unlike this, patientcentredness in the physician-oriented approach signifies "trust us; we know best what will help you" (Berwick, 2009, p. w558) with the belief that physicians always know what is best for their patients.

To attend those who suffer, a physician must possess not only the scientific knowledge and technical abilities, but also an understanding of human nature. The patient is not just a group of symptoms, damaged organs and altered emotions. The patient is a human being, at the same time worried and hopeful, who is searching for relief, help and trust. The importance of an intimate relationship between patient and physician can never be overstated because in most cases an accurate diagnosis, as well as an effective treatment, relies directly on the quality of this relationship. (Hellin, 2002, p. 452)

\footnotetext{
${ }^{21}$ Emotional intelligence refers to the ability to understand emotions of his/her-self and others, and regulates one's emotions and actions to increase the quality of life (Arora et al., 2010; Robbins \& Galperin, 2010).
} 
Patient-centredness is a patient's right which is core to healthcare, regardless of how it is called (Berwick, 2009). The first step in patient or person-centred care should start with an understanding of what matters to the patients (Rickert, 2012), and these matters should be considered by physicians according to their knowledge to benefit the patients (Berwick, 2009).

\subsection{Summary}

In this chapter, extant literature was reviewed in keeping with a grounded theory methodology and how the literature is interwoven into the emerging theory as data, in order to complete the theory. Accordingly, the literature review focused on the health-seeking behaviour of Korean immigrants in New Zealand and current understandings on relevant areas that can be compared and support the findings of the research. The literature review examined the following categories: migration and acculturation; immigrants' health and health service utilisation; immigrants' healthseeking behaviour and their illness perceptions; and patient's satisfaction with regard to quality of health services and person-centred care.

Immigrants' health-seeking behaviour is often considered a product of interactions between immigrants and the healthcare system in the host country. Immigrants shape their healthcare perceptions of the host health services depending on the healthcare they received, and their health-seeking behaviours are directly influenced by these perceptions. However, immigrants' healthcare perceptions are also influenced by their cultural backgrounds and the healthcare system that they are familiar with. Their illness perceptions are different to that of the locals in the host country. Their healthcare needs and expectations are largely unmet within the host healthcare system because they predominantly stem from their home country. They always compared their healthcare experiences in the host country to their previous healthcare experiences in their country of origin.

Some Koreans in New Zealand, who were eligible for free healthcare, visited Korea for healthcare despite putting their health at risks. Koreans' health-seeking behaviour in New Zealand is not well understood in the literature. To understand Koreans' health-seeking behaviours in New Zealand and their reasons for rejecting 
the New Zealand health services, I explored the literature related to patient's satisfaction, quality of health services, and person-centred care. Patients judge health service quality according to their level of healthcare satisfaction, and patient satisfaction is a subjective matter because it is heavily dependent on personal perspectives. Therefore, the quality of healthcare assessed by patients varies from one to another.

While the difference between the healthcare systems of the host and home countries is indicated as a determinant in immigrants' satisfaction with their healthcare, their healthcare satisfaction is also affected by the relationship between the immigrant patient and physician. The role of the interactions between patients and healthcare providers and healthcare provider's attitudes have been acknowledged as a major contributing factor to patient's satisfaction, and immigrant's healthcare satisfaction are no exception. Although the healthcare quality assessments of patients, which are considerably affected by their personal perceptions, have been subjected to question for their validity, patients' satisfaction, particularly based on the positive relationship with their physicians, resulted in improved physical and emotional healthcare outcomes. Patients who experience caring, empathic, reliable and responsive care express high levels of satisfaction, and physicians who empathise with their patients are more likely to be altruistic. Person-centredness based on the trust relationship between patient and physician becomes a major indicator in the quality of healthcare in health services. The following chapter provides detailed discussions pertaining to the issues around methodological choices and approaches of grounded theory as the research methodology for this research. 


\section{Chapter 3. Methodology}

\subsection{Introduction}

This research was designed to explore the healthcare experiences of Korean immigrants in New Zealand to understand their health-seeking behaviours and the factors affecting these behaviours. I decided to use grounded theory as a methodology for this research to find a latent pattern in Korean immigrants' healthcare experiences, behaviours, and perceptions in order to develop knowledge in a form of theory. The chapter begins with the fundamental characteristics of qualitative research, followed by a brief history of grounded theory and its divergence. The overview of grounded theory includes its essential tenets which are the congruent, iterative and integrative data collection and data analysis, and the constant comparison with theoretical sampling. This chapter demonstrates methodological choices and challenges I experienced throughout the research process. Having chosen grounded theory as the methodology, the dilemma was choosing which version of grounded theory would best suit the research. I intended to follow Charmaz's constructivist version of grounded theory at the beginning of the research, but moved to Glaser's classic grounded theory during the research. The reasons for the change and a rationale and justification of the classic grounded theory for this research are explained in detail. I also discuss the difficulties in adopting grounded theory that researchers may experience, such as choosing one version of grounded theory from the various versions, the different roles of the literature review, and the unfamiliar attributes of grounded theory as a general methodology.

\subsection{Qualitative research approach}

Qualitative inquiries originated from hermeneutics, phenomenological sociology, and the Verstehen (understanding and interpretation) tradition aimed to understand human behaviours and interpret the meanings of life that people make of their experiences (Schwandt, 2007). Researchers who focus on human behaviours or meanings, employ a qualitative research approach to explore and observe the issues 
of the study phenomenon in a natural setting (Hesse-Biber \& Leavy, 2010; Sullivan \& Ebrahim, 1995). Qualitative research is the most suitable approach to study the "process" of social phenomenon rather than its outcomes because:

(1) depicting process requires detailed description of how people engage with each other, (2) the experience of process typically varies for different people so their experiences need to be captured in their own words, (3) process is fluid and dynamic so it can't be fairly summarized on a single rating scale at one point in time, and (4) participants' perceptions are a key process consideration. (Patton, 2001, p. 159)

Unlike researchers undertaking quantitative research, who see subjectivity as sources of bias, researchers using qualitative methodologies pay more attention to subjective reality over the objective truth, and believe that the subjective reality constructed by individuals or groups of people is also truth (Polit \& Beck, 2013). Qualitative researchers are interested in diversity in social phenomenon rather than its universality, and listen to people's stories and experiences that they had not heard or understood yet (Auerbach \& Silverstein, 2003).

There is a dearth of understanding on the health-seeking behaviour of Korean immigrants in New Zealand and their healthcare adapting process, which requires new knowledge and theories to explain them. In order to understand the main healthcare concerns of Korean immigrants in New Zealand and to find a grounded pattern in their behaviour; Korean immigrants' healthcare experiences in both Korea and New Zealand, including their health beliefs and healthcare perceptions, were explored. Therefore, an exploratory qualitative research design was adopted to look closely at Koreans' health-seeking behaviour to understand what is going on. By employing a qualitative approach, Korean immigrants (who are a minority ethnic group in New Zealand and somewhat marginalised in the healthcare system) talked about their personal experiences and thoughts with respect to health and healthcare. To shed light on the specific social process they underwent, and to develop knowledge in a form of theory based on the perspectives of Korean immigrants, grounded theory was chosen as the research methodology for this research.

Particularly, Glaser's classic grounded theory (Glaser \& Holton, 2004), which is the 
original version of grounded theory, was the selected methodology. I adopted grounded theory as a neutral/general methodology beyond the quantitative and qualitative paradigms (Holton, 2008); therefore, there are no philosophical assumptions or theoretical paradigms claimed for this research.

\subsection{Grounded theory}

\subsubsection{An overview}

Grounded theory is an inductive methodology which refers to "the discovery of theory from data systematically obtained from social research" (Glaser \& Strauss, 1967, p. 2). This methodology is a theory developing methodology, not to test or verify existing theories (Charmaz, 2014; Corbin \& Strauss, 2008; Glaser \& Strauss, 1967; Mills et al., 2006). Grounded theory offers a freedom to enter the empirical social world to discover or generate a theory grounded in participants' daily life (Jones \& Alony, 2011; Mills, Bonner, \& Francis, 2006). Researchers collect and analyse data with an open mind and as minimised preconceptions as possible, in order to generate theory naturally from the data without forcing or contamination (Glaser, 2013). Grounded theory emerged from the symbolic interaction tradition of social psychology and sociology. It emphasises people's actions based on the meanings that they develop, share, and rely upon in the process of social interaction; for example, words, expressions, and signs (Blumer, 1986). This methodology is well suited to a research with complex human behaviours and underlying social issues affecting people's lives (Charmaz, 2014; Lingard, Albert, \& Levinson, 2008). It aims to provide a theoretical explanation of a social process (not structure) through a theory generated from data (Charmaz, 2014; Corbin \& Strauss, 2008; Glaser \& Strauss, 1967; Mills et al., 2006). Grounded theory is the most appropriate methodology to research when there is a dearth of knowledge or no theory to explain the issues of the phenomenon being studied (Birks \& Mills, 2010; Creswell, 2012; Crooks, 2001; Glaser, 1992).

The most salient distinctions of grounded theory compared to other qualitative methodologies are constant comparative analysis together with theoretical 
sampling in its theory generating process (Bryman, 2008; Glaser \& Strauss, 1967; Hallberg, 2006). Data collection and analysis in grounded theory is a simultaneous and iterative ongoing process. Once data are collected from interviews and start to generate theoretical ideas, the next data collection is informed by these ideas; that is, theoretical sampling is applied and directs further data collection with theoretical sensitivity as a part of the theory generation process (Charmaz, 2014; Corbin \& Strauss, 2008; Glaser \& Strauss, 1967). According to Dey (1999), theoretical sampling helps "the researcher to identify possible areas of inquiry... without any presumption about the nature of the relationship that may hold" (pp. 170-171). At the same time, the researcher consistently compares between emerging incidents ${ }^{22}$, incidents with each category ${ }^{23}$, and between categories (Charmaz, 2014; Glaser, 2002; Glaser \& Strauss, 1967). As a result, the researcher is able to define the basic properties $^{24}$ of each category and refine the categories as well. In keeping with constant comparative analysis and theoretical sampling during the data collection and analysis, theory generation is closely connected to the data because there are fewer chances of involvement with researcher's assumptions; therefore, a theory can be developed with theoretical sensitivity (Bryman, 2008; Glaser \& Strauss, 1967). The constant comparisons and theoretical sampling continue until a strong theoretical understanding is achieved and no new theoretical idea emerges from newly collected data (Bryant \& Charmaz, 2010; Corbin \& Strauss, 2008).

\subsubsection{History and divergence of grounded theory}

Grounded theory has been developed and expanded from the 1960s. This methodology was originally developed by two American sociologists, Barney Glaser and Anselm Strauss from their research on death and dying patients in hospitals (Charmaz, 2014). Glaser and Strauss defined and explained grounded theory as a research methodology in their publications: Awareness of Dying (1966) and The Discovery of Grounded Theory (1967). They offered a set of rigorous research

\footnotetext{
22 Incidents are data and indicators of a category or concept (Bryant \& Charmaz, 2010).

${ }^{23}$ Category can be referred to as class or division distinguished by similarities and differences between groups (Dey, 1999). It is also seen as a concept (Glaser, 1992).

${ }^{24}$ Property is an attribute or quality of a thing that is owned by a category (Dey, 1999).
} 
procedures of data analysis and theory generation for systematic qualitative research, and showed that the outcomes of qualitative research projects could be as significant as quantitative research projects (Bryant \& Charmaz, 2010). This inductive research approach (explaining issues of the research through a theory generated from data), challenged the dominant deductive quantitative research paradigm (focusing on preexisting theory testing) at that time (Charmaz, 2000; Charmaz \& Henwood, 2008). Grounded theory became one of the most popular methodologies in qualitative research (Birks \& Mills, 2010).

Glaser and Strauss' different academic backgrounds contributed to a divergence of grounded theory in later decades (Charmaz, 2014). While Strauss was significantly influenced by the traditions of pragmatism and symbolic interactionism from the University of Chicago ${ }^{25}$, Glaser's background was strongly rooted in rigorous quantitative methods at Columbia University (Bryant \& Charmaz, 2010; Dillon, 2012). Their different interests and ideas of grounded theory, which were influenced by their educational backgrounds, conflicted. Finally, in the late 1980s, Strauss, together with Corbin, developed a new version of grounded theory called "Straussarian" (Corbin \& Strauss, 2008, 2014; Strauss \& Corbin, 1994, 1998). They adopted detailed analytic techniques and more prescriptive procedures, and moved grounded theory toward theory verification. Glaser criticised the contradictive components in Strauss and Corbin's new version of grounded theory which he argued resulted in forced data and preconceived categories. Glaser strongly emphasised "theory emergence" with theoretical sensitivity in grounded theory, and stayed with the original grounded theory which is known as "Glaserian" or “classic/traditional/pure grounded theory” (Glaser, 1992).

Heath and Cowley (2004) pointed out that the main reason for this discrepancy was not philosophical but methodological. While Strauss and Corbin (1998) believed they progressed grounded theory, Charmaz (2014) criticised their verification and positivistic underlying assumptions. More recently, Corbin updated

\footnotetext{
${ }^{25}$ The Chicago School qualitative traditions associated with naturalistic observational approach, pragmatist assumptions, and symbolic interactionist perspective. It took into account language, meanings, and actions to understand human's life and experiences (Bryant \& Charmaz, 2010).
} 
her earlier work ${ }^{26}$ with Strauss; she added an explanation of pragmatism and symbolic interactionism as the philosophical underpinning for their version of grounded theory which were absent from the earlier edition (Corbin \& Strauss, 2008). From the 1990s, Glaser and Strauss' grounded theory has evolved or remodelled because of researchers, such as Charmaz, with a different ontological and epistemological stance in terms of different philosophical perceptions (Berterö, 2012; Mills et al., 2006).

There was considerable debate and criticism towards Glaser and Strauss' Grounded theory methodology. Birks and Mills (2010) and Bryant and Charmaz (2010) claimed that it is not a methodology as it does not offer philosophical and theoretical paradigms which orient the stance of researcher and the researched. Rather it is a method or an approach with various strategies and techniques. Charmaz (2014) has criticised its positivistic assumptions with an objective reality and the distance between researcher and participants to prevent the subjective biases of the researcher. Charmaz moved away from the original grounded theory and developed a constructivist grounded theory; it applies the Glaser and Strauss' traditional grounded theory approach but takes a more subjective and flexible stance within a constructivist paradigm, and denies an objective position and an emergence of theory (Berterö, 2012; Charmaz, 2014; Mills et al., 2006). Charmaz's constructivist grounded theory takes relativism as an ontological stance and chooses subjectivism as an epistemological stance; it emphasises an interrelationship between researcher and participants, and focuses on the subjective role of the researcher. She articulated, "Research acts are not given; they are constructed. Viewing the research as constructed rather than discovered fosters researchers' reflexivity about their actions and decisions" (2014, p. 13).

Sociologist Clarke (2005), who was a student of Strauss, reshaped grounded theory further based on a postmodernist approach and developed situational analysis. She focuses on the importance of using situations rather than actions or processes,

\footnotetext{
${ }^{26}$ After Strauss died in 1996, Juliet Corbin published two more editions $\left(3^{\text {rd }}\right.$ and $\left.4^{\text {th }}\right)$ of Basics of qualitative research: Techniques and procedures for developing grounded theory (Corbin \& Strauss, 2008, 2014) which was the original work with Strauss (Strauss \& Corbin, 1994, 1998).
} 
and provides practical tools such as situational maps to analyse data (Allen, 2010). However, she is criticised for her version of grounded theory because it incorporates Strauss' work (Morse, 2009), and it is doubted that using her situational analysis approach will allow data to emerge (Allen, 2010). Schatzman (1991), a colleague of Strauss and Glaser in the 1960s-1970s, developed a dimensional analysis as an alternative theory generation method. He viewed theory generation in the original grounded theory as lacking a structural analytical foundation, and proposed a dimensional analysis method with a clearly articulated analytic process within a structural foundation (Berterö, 2012). Theory in dimensional analysis aims to explain a complex social phenomenon related to its multiple dimensional attributes rather than a basic social process alone (Kools, 1999; Kools, McCarthy, Durham, \& Robrecht, 1996). However, this method is limited and rare in the literature (compared to other versions of grounded theory) because it has been less documented (Morse, 2009).

\subsection{Rationale for classic grounded theory}

The central concern regarding methodological inquiry of this research was how to explore and examine Korean immigrants' issues in healthcare and their health-seeking behaviours in New Zealand. The uncertainty was not the only issue of the research, there was also an issue with the methodological approach to conduct the research. As indicated in the introduction, Charmaz's constructivist grounded theory was the chosen version of grounded theory for this research in the first place, but I changed to Glaser's classic grounded theory because of the discrepancy between the research and the methodology. This was due to the following understandings; philosophical assumptions with respect to grounded theory, researchers' role including the relationship with participants, and the ways of theory generation in grounded theory.

\subsubsection{General methodology grounded theory}

Creswell (2012) stated, "researchers bring their own worldviews, paradigms, or set of beliefs to the research project, and these inform the conduct and writing of 
the qualitative study" (p. 15). Researchers choose a version of grounded theory based on their philosophical or theoretical perspective, and the selected version of grounded theory underpins and directs the researcher and the research (Jeon, 2004). Choosing constructivist grounded theory as a research methodology for my research was largely due to its clearly defined philosophical underpinnings. I was attracted to the constructivist philosophical assumptions. Constructivist grounded theory takes, ontologically a relativist stance (there is no absolute truth rather the shared reality constructs truth), epistemologically a subjectivist stance (the emphasised subjectivity in the role of the researcher), and methodologically an interpretivist stance (the mutually constructed reality is interpreted by the observer) (Gardner et al., 2012). Particularly, as a novice researcher, this articulated philosophical foundation in constructivist grounded theory seemed clear to understand and easy to follow its methodological process; because it provided principle guidance in how to look and think, how to interact with the data, and how to interpret them. However, as the research progressed and my understanding of grounded theory grew, I started to doubt whether the chosen constructivist grounded theory was the right version of grounded theory. I confronted the nature of grounded theory (I understood it as a neutral and general methodology) and questioned the influences of the researcher's philosophical assumptions that I adopted. I was able to see that this research was better suited to classic grounded theory than constructivist grounded theory.

Researchers inexperienced in grounded theory are expected to encounter fear and confusion when they analyse data at the beginning of the research (Glaser, 2002, 2011). Initially, I was confused with grounded theory because of my lack of confidence in using this methodology. I also doubted the data due to their scattered meanings and disconnections between them at the beginning. However, once data started to reveal some theoretical meanings and ideas, even though it took time, the earlier meaningless ideas made their own sense, and then the research was directed by the emerging concepts and categories from the data. The properties and concepts were developed and saturated during the ongoing data collecting and analysis process. Particularly, using the constant comparative analysis with the theoretical sampling helped me to conceptualise theory with theoretical sensitivity close to the 
data. It was also expected by Glaser (2002) that the confidence and ability of the researcher would grow as the experience of grounded theory grew.

The research was directed by its own theoretical guidance which directly emerged from the data, not the philosophical assumptions of constructivist grounded theory. I realised that grounded theory, as a methodology, does not need to have a specific philosophical assumption or theoretical paradigm to guide research. Glaser (2010) articulated that grounded theory is an independent methodology with "a total package" (p. 3). Grounded theory, particularly original (classic) grounded theory, is a general methodology that "stands alone on its own, as a conceptualizing methodology" (Glaser \& Holton, 2004, p. 55). Classic grounded theory resists being confined by any specific philosophical perspectives, and also rejects being either positivist (quantitative) or interpretivist (qualitative) in paradigm; rather this methodology acknowledges its roots in both quantitative and qualitative paradigms (Holton, 2008; Simmons, 2011). Simmons argued that it is "not legitimate justification for choosing to purposefully incorporate one's theoretical paradigm, political or professional ideology, theoretical speculations, and/or personal predilections into the research" (p. 4). Research should be guided by its own purposes and needs rather than its chosen philosophical stances (Glaser, 2001).

\subsubsection{The role of the researcher}

Constructivist grounded theory shrinks the distance between researcher and participants; the researcher's active involvement in theory construction is required as a significant part of the theory building (Charmaz, 2014). Constructivist grounded theorists often regard the researcher as a "passionate participant" (Denzin \& Lincoln, 2005, p. 196). One of the most distinctive differences between constructivist and classic grounded theory is the role of the researcher in theory generation. Charmaz, who remodelled original (classic) grounded theory to a constructivist version with articulated philosophical assumptions, believes that theory co-construction between the researcher and participants is crucial (Glaser, 2012). She emphasises the role of the researcher as a theory co-constructor together with participants (Gardner et al., 2012; Mills, Chapman, Bonner, \& Francis, 2007). Charmaz denies the distance 
between a researcher and participants, and criticises the role of the researcher in classic grounded theory as a "distant expert" (Charmaz, 2000, p. 513) or a "neutral observer" (Charmaz, 2014, p. 343).

While the distant experts/neutral observers in classic grounded theory keep a distance from the participants in order to avoid researchers' subjective biases (Charmaz, 2014), the theory co-constructor researcher in constructivist grounded theory actively interacts with his/her participants and participates in the theory construction as a passionate participant (Gardner et al., 2012; Mills et al., 2007). She emphasises the subjectivity of the researcher and ignores the objectivity in the role of the researchers, and claims that the complete objective stance of the researcher is far from reality (Mills et al., 2006). Glaser (2012) raised concerns towards the mutual theory construction between a researcher and participants in constructivist grounded theory. In his view, the researcher's empowered subjectivity and their active involvement in theory construction would result in weakening the voices of the participants but raising the voice of the researcher. Consequently, the researcher's influence on theory generation would be greater than that of participants. He pointed out the notion of theory co-construction or co-creation in constructivist grounded theory as a "legitimate forcing" (Glaser, 2012, p. 31), whereas Charmaz (2014) argued that "the theory depends on the researcher's view; it does not and cannot stand outside of it" (p. 239). Charmaz (2014) claimed Glaser's (classic) grounded theory was objectivist grounded theory which limits the researchers' understanding of the participants' subjective world. Simmons (2011) questioned Charmaz's constructivist-objectivist dichotomy, and argued that classic grounded theory is a neutral and general methodology with the elements of both constructivist and objectivist, so it is not appropriate to clarify it into either constructivist or objectivist.

The empowered subjective role of the researcher, I thought, would be more helpful for a researcher because it may give better chances to look closely at the issues of the Korean immigrants, and it would result in achieving a deeper insight into that issue. So I acknowledged the researcher's subjective role and its influences on research, but I was not sure to what extent the researcher's subjectivity in constructivist grounded theory was allowed. Interviewing the participants as a 
researcher allowed me to enter the participants' subjective world (Charmaz, 2014). I could explore each Korean participant's personal experiences, beliefs, and perceptions and expectations with respect to their health and healthcare. Being a Korean researcher myself and interviewing Korean participants in their homes almost automatically generated an intimate relationship between the researcher and the participants. They often asked my opinions about their experiences and thoughts. I was not the only one observing at the interview, the participants also observed the researcher. I had to inform the participants of the role of the researcher as an interviewer in order to have them focus on their experiences and stories not the researcher's. Once the participants understood me as a researcher who listens to them but did not answer, their stories were deepened with fewer interruptions. I had to admit that maintaining a certain amount of distance (in a flexible manner) between the researcher and participants was necessary, and it was somewhat beneficial to obtaining quality data. On the other hand, sharing the same background with the participants and speaking the same language helped me to have a better understanding of the participants' experiences, and assisted the participants to stay open to me as well. I understand that the role of the researcher in grounded theory is not about the complete subjective or objective role because it is impossible and is not necessary. Researchers who claim to be absolutely objective are questioned about their "unconscious biases" and researchers who claim to be completely subjective are asked about the bias contaminated by their perceptions (Markey et al., 2014, p. 20). If "we are part of the world" as Charmaz stated (2014, p. 17), we cannot avoid both the subjective reality and the objective truth, I think.

\subsubsection{Theory generation}

Although grounded theory methodology offers researchers analytic power to generate theory (Charmaz, 2014), the approaches to theory generation vary according to the versions of grounded theory, particularly between classic and constructivist grounded theory. The theory in classic grounded theory emerges directly from the data (participants) without forcing (Glaser \& Strauss, 1967), while constructivists believe that theory is constructed by a mutual understanding between the researcher and participants (Charmaz, 2014). According to Charmaz (2000), "Data do not 
provide a window on reality. Rather, the 'discovered' reality arises from the interactive process and its temporal, cultural, and structural contexts" (p. 524). Glaser (2002) views the co-constructed theory between researcher and participants as a forced theory, and claims that "the participants are the data, NOT the theorists. The participants, while having great involvement in resolving their main concern, seldom have a conceptual perception of it as a GT theorist does" (p. 29). It is strongly believed that any theory emerged from the data (through the constant comparative analysis with theoretical sampling) will fit and work ${ }^{27}$; the theory is able to explain the issues of the research, if not the theory is forced (Glaser \& Strauss, 1967). Classic grounded theory aims to generate a conceptual theory to provide conceptual explanations of the studied phenomenon beyond the descriptive or interpretive explanations (Glaser, 2012; Glaser \& Strauss, 1967). Researchers, to generate a conceptual theory, need to understand "not only what is being told, how it is being told and the conditions of its being told, but also all the data surrounding what is being told" (Glaser, 2012, p. 29).

The theory generated from this research was not mutually constructed by the researcher (myself) and the participants, but emerged from the participants as guided by the constant comparative analysis and theoretical sampling. Participants shared their personal healthcare experiences and their thoughts with me. I gathered data from Korean participants' experiences and stories like pieces of a puzzle, and worked with all the pieces of data together until a picture was formed. I was able to see the reality constructed by the participants themselves (even if participants were not able to see such). There was limited interaction between the researcher and the participants; "The GT researcher listens to participants venting issues rather than encouraging them to talk" (Glaser \& Holton, 2004, p. 57). Participants who had experienced and/or are experiencing the phenomenon were the experts, not the researcher (Auerbach \& Silverstein, 2003). The theory generated from my research was one that emerged and was discovered not constructed, so I moved from Charmaz's constructivist version of grounded theory to Glaser's classic grounded theory.

\footnotetext{
${ }^{27}$ See the Section 4.9 Data analysis (p. 99).
} 
To sum up, there were several reasons to use classic grounded theory for this research. First, this research was guided by the emerging data, not the researcher's personal philosophical perspectives. Second, the role of the researcher was that of a neutral observer rather than a theory co-constructor. Thirdly, the substantive theory emerged from the data and was not mutually constructed by the researcher and the participants. Finally, in order to render a conceptual understanding of Korean immigrants' health-seeking behaviour in New Zealand, the data analysis in this research was focused on a conceptual abstract level beyond the descriptive or interpretive level. I looked at the data with distance and closely, and analysed the data subjectively and also objectively.

\subsection{Challenges and difficulties}

Grounded theory is a well-known systematic theory generating research methodology and has been widely used in qualitative research (Bulawa, 2014). However, researchers, particularly novice researchers, are often confronted with the challenges of difficulties in conducting grounded theory and also confusion due to the various versions of grounded theory (Cho \& Lee, 2014; Elliott \& Higgins, 2012; Holton, 2008; Kwok, McCallin, \& Dickson, 2012; Tan, 2010). According to Markey et al. (2014), researchers are able to choose one of the versions of grounded theory according to their philosophical beliefs and the characteristics of the research. Glaser and Holton (2004) emphasised that "Grounded theory deals with the data as it is" (p. 50). The question is: how does the researcher know that the chosen version of grounded theory is the right one for the research if one does not know what the data are (before the data are collected)?

\subsubsection{Various versions of grounded theory}

There is no one way to conduct grounded theory; it varies depending on the researchers and according to the versions of grounded theory (Bulawa, 2014). Most researchers planning to undertake a grounded theory research face the dilemma of choosing which version of grounded theory best suits their studies (Bulawa, 2014; Markey et al., 2014). As the various versions of grounded theory share many 
common elements but with different perspectives (Charmaz, 2014), researchers who have different philosophical stances have moved from the original grounded theory and developed other versions of grounded theory (e.g., Strauss and Corbin's Straussarian, Charmaz's constructivist grounded theory, Clarke's situational analysis, and Schatzman's dimensional analysis). Researchers who are new to grounded theory can be easily confused by the similar but different versions of grounded theory approaches and the ongoing debates over grounded theory amongst these versions (Bulawa, 2014; Markey et al., 2014). Researchers are required to understand the methodological distinctions and the different philosophical underpinnings between the versions of grounded theory approaches to enable them to select one for their studies and also to justify their chosen versions (Kenny \& Fourie, 2015).

However, grounded theory is not easy to understand and learn if one does not have actual experiences in grounded theory (Echevarria-Doan \& Tubbs, 2005; Kwok et al., 2012). For example, the theory "emergence" is a fundamental principle in classic grounded theory, accordingly researchers adopting a classic grounded theory methodology must trust in theory emergence (Glaser \& Holton, 2004); "trust that emergence will occur and it does" (Glaser, 1992, p. 4). However, theory emergence seems difficult to understand without any experience, and it cannot be trusted if one cannot understand it. Researchers are also asked about "an ability to conceptualize, to organize, to tolerate confusion with some incident depression, to make abstract connections, to remain open, to be a bit visual, to thinking multivariately and most of all to trust to preconscious processing and to emergence" (Glaser, 2009, p. 2). Researchers inexperienced in grounded theory may not know if they are capable of all this; further, they may not even understand what the above means. Researchers find grounded theory hard to understand until the procedure is finished (EchevarriaDoan \& Tubbs, 2005; Kwok et al., 2012). Glaser (2009) sees these experiences of the researcher (confusion and struggles in conducting the methodology) as part of the grounded theory procedure, and he advises that they (confusion and struggles) should be tolerated for a while. A number of classic grounded theorists suggest that, from experience, the best way to learn grounded theory is to "just do it" and it can be learned (Elliott \& Higgins, 2012; Glaser \& Holton, 2004; Kwok et al., 2012; Ng \& Hase, 2008; Pergert, 2009; Roderick, 2009). 


\subsubsection{Methodological confusion}

Grounded theory, a general methodology, stands alone separated from other methodologies (Glaser \& Holton, 2004; Holton, 2008; Kwok et al., 2012). It is applicable not only when using qualitative data but also to the use of quantitative data, and has both objectivist and constructivist perspectives (Holton, 2008). However, researchers easily confuse grounded theory with qualitative research methodologies due to its similar methodological procedures and components; for example, data collection from in-depth interviews, observations, and field notes, and inductive data analysis (Cho \& Lee, 2014; Glaser \& Holton, 2004; Holton, 2008). The terms used in grounded theory and qualitative research are also similar, such as explore, open-ended question, stories, themes, concepts, and theories (Dey, 1999). Accordingly, grounded theory is often classified as a qualitative methodology and adopted as one of the qualitative methodologies or methods, and it is a common misunderstanding amongst qualitative researchers (Glaser \& Holton, 2004; Holton, 2008). This methodological confusion has led researchers to modify original grounded theory to another version with a qualitative research orientation (Glaser \& Holton, 2004; Holton, 2008; Simmons, 2011). Theoretical paradigms or philosophical assumptions are adopted into the general grounded theory; and it resulted in, losing the key attributes of the original grounded theory such as theory emergence and theory conceptualisation (Glaser \& Holton, 2004). In this perspective, the other modified versions of grounded theory that moved from the original grounded theory to the qualitative territory with qualitative perspectives are considered as "remodelled" rather than "evolved" (Glaser \& Holton, 2004; Simmons, 2011).

\subsubsection{Research question}

In general, research begins with a research question and the research question identifies issues and scopes of the research (Flick, 2008). However, stating a research question at the beginning of the research is not recommended in grounded theory, rather it is preferred to have a research question that is generated from the data during the research (Glaser \& Holton, 2004; Glaser \& Strauss, 1967). The research 
question in grounded theory is formulated according to data generated during the research and is refined and focused as the research progresses. As Glaser and Strauss (1967) emphasised, "The initial decisions are not based on a preconceived theoretical framework" (p. 45), grounded theory studies start with a broad and general research question that indicates general interests of the research not researchers' preconceived ideas or existing theories (Dey, 1999; Willig, 2013). So, the research question does not influence the theory generating process and confine the scope of the research.

However, Strauss who remodelled grounded theory to another version (Straussarian) with Corbin (1998) changed his view on the research question, and emphasised the need for the research question from the early stages of the research. According to Strauss and Corbin, the research question with specific theories or hypotheses provides boundaries of the research and it helps researchers to focus on the topic of the research. Glaser and Holton (2004) argued that the research question with preconceived ideas (extant theories or hypotheses) will limit the researchers' flexibility and freedom to explore the phenomenon being studied and will force the process of theory generation. Flick (2008) stated, it seems easier and helpful for researchers, particularly for novice researchers, to be guided by a clear research question with defined scopes of the research. Even so, the decision regarding the research question in grounded theory (whether it directs the issues of the research or the general interests of the research) will be left to the researcher, and the researchers have to make a decision according to the versions of grounded theory they choose to adopt (Birks \& Mills, 2010).

\subsubsection{Literature review: When and how}

In traditional qualitative research, a literature review at the early stage of the research provides the relevant knowledge related to the research topic, discovers gaps in the current knowledge, and helps to develop the research questions or hypotheses (Birks \& Mills, 2010; Mavetera \& Kroeze, 2009). However, the role of the literature review and when to conduct this in grounded theory differs to those of other research methodologies (Andrew, 2006). Reviewing the existing literature in grounded theory aims to support and explain the theory, not to find a research gap in 
the literature (Chen \& Boore, 2009; Ng \& Hase, 2008). Thus, searching for the relevant literature is delayed until the data collection and analysis is finished (Mavetera \& Kroeze, 2009). Glaser and Holton (2004) strongly articulate that "The mandate is to remain open to what is actually happening and not to start filtering data through pre-conceived hypotheses and biases to listen and observe and thereby discover the main concern of the participants in the field" (p. 57). The delayed literature review in grounded theory serves to prevent the researcher's preconceived ideas, derived from the literature, having an influence on data, and to ensure theory emerges from the participants, not from the researcher or from the existing theories and literature (Chen \& Boore, 2009; Markey et al., 2014).

However, there is a debate about the literature review in grounded theory, when to conduct it and to what purpose, amongst grounded theorists (Birks \& Mills, 2010; Bluff, 2005; Chen \& Boore, 2009; Ng \& Hase, 2008). Bluff (2005) argues that delaying the literature review does not promise that the theory generation is free from the researcher's preconceived ideas. For example, if the researcher is somehow related to the phenomenon being studied, their knowledge and experience would influence the data regardless of the influences of the literature review. Clegg (2003) also argues that if the research area is new there would be a lack of literature to review; accordingly, the literature review at the beginning of the research is less likely to impact on the research. On the other hand, the delayed literature review is supported by Gilgun (2014). She pointed out the advantage of the delay; according to her, the literature review conducted after the data analysis has a stronger connection with the findings of the research rather than the literature reviewed conducted at the early stage of the research. Strauss and Corbin (1998) recommend an on-going literature review at all stages of the research. They acknowledge the researcher's inevitable influences on the research and also the benefits of the literature review during the research as it provides an analytic tool to stimulate the researcher's theory conceptualization.

Locating the literature review and the research question in grounded theory is an ongoing debate, and it is generally a matter of which version of grounded theory. However, researchers are, regardless of the version of grounded theory they adopted, 
required to address and identify the gaps within the academic knowledge and define a research question to justify the needs for the research (Birks \& Mills, 2010; Markey et al., 2014). Therefore, ignoring prior review of the literature would be problematic for most researchers because they do not have sufficient knowledge of the research area they have proposed at the beginning (Birks \& Mills, 2010). In this research, I carried out two different literature reviews at different stages with different objectives. The first literature review took place at the beginning of the research to learn what is known and not known about the health-seeking behaviour of Korean immigrants in New Zealand in the literature, in order to identify the knowledge gap and get approval for the research. The second review was undertaken when data were almost analysed and the theory was nearly formulated to fill the gap in the research findings and to complete the theory as data.

\subsection{Quality of grounded theory}

Addressing rigour is a crucial component in qualitative studies to articulate the integrity of the research process and the accuracy and competency of research findings (Aroni et al., 1999; Charmaz, 2014; Rambaree, 2007). As qualitative research methodologies/methods are flexible guidelines, there is no one approach that guarantees rigour in qualitative studies and no set criteria that applies to all qualitative studies (Charmaz, 2014; Corbin \& Strauss, 2008; Gasson, 2004; Rolfe, 2006). The degree of rigour in a qualitative research varies depending on the researcher, the research purposes, and the methodologies/methods employed in the research (Charmaz, 2014; Corbin \& Strauss, 2008). The rigour in grounded theory studies is often criticised and doubted due to its inductive theory generation approach, theoretical saturation which relies on the research's subjective judgements, and biases influenced by the researcher's subjective stance (Gasson, 2004). Gasson argues that criticism and suspicion of grounded theory studies is due to its assessments of rigour based on a positivist view rather than an interpretive view. Grounded theory studies aim to derive theory from data, not to test existing theory (Charmaz, 2014; Glaser \& Strauss, 1967); therefore, judging the rigour of a grounded theory research via positivist evaluations is not right (Gasson, 2004). The rigour of a grounded theory research should be measured by the manner of explanation of the 
research process and theorising of the phenomenon, but not the research findings alone (Hammersley, 1987).

Charmaz (2014) provides a set of multiple criteria for ensuring rigour in grounded theory studies: credibility, originality, resonance, and usefulness. She emphasises the use of multiple validity strategies to check the accuracy and credibility of research findings. While Corbin (Corbin \& Strauss, 2008) sees Charmaz's criteria as comprehensive which include both the scientific and creative aspects of a qualitative research, Glaser and Holton (2004) draw attention to the concerns on accuracy and descriptive capture in Charmaz's criteria. Glaser and Holton argued that they (accuracy and descriptive capture) are the pure qualitative researchers' concerns, not for the grounded theory researchers. According to them, grounded theory is about conceptual theory not accurate descriptive theory; therefore, these concerns have nothing to do with the rigour of grounded theory. Adopting qualitative research criteria to measure a grounded theory research quality with the concerns of accuracy and descriptive details will result in losing the analytic power of grounded theory. As Charmaz (1990) articulated in her early publication, "The rigor of the grounded theory method depends upon developing the range of relevant conceptual categories, saturating (i.e. filling, supporting, and providing repeated evidence for) those categories, and explaining the data" (Charmaz, 1990, p. 1163). Therefore, the quality of grounded theory studies varies according to the methodological thoroughness of the research; that is, the quality of grounded theory research varies depending on how researchers conduct the research (Holton, 2008). Grounded theory, a general methodology, is an independent methodology with its own systematic process of sampling, coding, and memoing (Jones, 2009). It is not appropriate to assess the quality/rigour of grounded theory by the external criteria, but it must be measured by its own criteria of the methodology (Charmaz, 1990).

Glaser (1978) offered four criteria to evaluate the quality in grounded theory studies; fit, work, relevance, and modifiability. The criterion fit is a fundamental component of theory generation; fitting data into categories and categories into data, and later fitting categories into a theory. It enables a theory to fit into the phenomenon being studied. Work is about the applicability of the theory. Theory 
should explain and interpret what is going on in the area under research. Relevance means the degree of understanding of the substantive research area; the theory must focus on the main concerns or core problems under research. Modifiability implies continuous theory modifying; this means the theory should be modifiable with fresh ideas from new data (Glaser, 1978; Glaser \& Strauss, 1967). Glaser's criteria help the researcher to be aware of how his/her theory is constructed and how the constructed theory works (Charmaz, 2014). The quality of grounded theory assures application of the theory emerging from the research in its own substantive area and beyond (Glaser \& Strauss, 1967).

\subsection{Summary}

This exploratory qualitative research adopted grounded theory as a research methodology to develop a conceptual theory of the health-seeking behaviour of Korean immigrants in New Zealand. In this chapter, I explained my methodological choices which were based on the characteristics of the research, and shared information to help readers understand the methodology that I chose to employ for this research. I have provided an overview of qualitative research and grounded theory including its background and divergence. It was followed by a detailed discussion on the choice of grounded theory, specifically Glaser's classic grounded theory as the suitable research methodology for this research. I described the challenges and difficulties in conducting grounded theory that I encountered during the research. These challenges and difficulties were mainly due to the various versions of grounded theory; the different roles and different approaches to the literature review in grounded theory; and the characteristic of grounded theory as a general methodology. I have introduced Glaser's four criteria (fit, work, relevance, and modifiability) to maintain and evaluate the rigour and quality of grounded theory studies, and reasoned why the rigour of grounded theory should not be judged by other qualitative research criteria. The following chapter details how these features of grounded theory were applied during the research and how they influenced the theory generating process in this research. 


\section{Chapter 4. Methods}

\subsection{Introduction}

Grounded theory provides tools to conduct research but these tools are simply techniques to use, not instructions to follow (Charmaz, 2014). Keeping a "keen eye, open mind, discerning ear, and steady hand" (p. 26) will render insights into the phenomenon being studied. The previous chapter "Methodology" explained the issues with the methodological choices and challenges experienced in this research. In this chapter, the theory generating process is introduced with a step-by-step description of the procedure undertaken throughout the research, along with a detailed explanation of how this procedure was guided by Glaser's classic grounded theory methodology.

It begins with the sampling criteria applied to recruit participants and the ethical considerations associated with the participants interviewed. The detailed explanations of the research setting, recruitment, sampling methods, and participant's characteristics are provided. The data collection and analysis processes are also discussed along with the constant comparative analysis together with theoretical sampling. I address the difficulties and advantages of carrying out a grounded theory research in a second language through my personal experience, and introduce how I managed rigour in this grounded theory research.

\subsection{Sampling criteria}

Immigrants' health-seeking behaviours vary not only amongst immigrant ethnic groups but also within an ethnic group depending on their immigrant status and levels of adaptation. Accordingly, there are certain factors that directly affect immigrants' health-seeking behaviour, such as duration of residency, language proficiency, physical status, socio-economic and immigration status, and cultural background (Choi, 2013a; Davies et al., 2009; Zimmerman et al., 2011). Therefore, certain criteria for the participant recruitment were necessary to find specific embedded factors affecting Korean immigrants' healthcare decisions and their 
health-seeking behaviours in New Zealand. The sampling criteria applied in this research were: aged 18 or over (to have healthcare experience independent of their parents); duration of residency in New Zealand of 18 months or more (time to understand and experience health services in New Zealand); and being a resident, permanent resident or citizen of New Zealand, or holding a work permit or student visa (eligible for the same healthcare benefits as locals). Research participants were recruited according to these criteria.

\subsection{Ethical considerations}

When conducting any research the researcher has an obligation to be aware of the possible consequences which may be harmful to participants (Drew, Hardman, \& Hosp, 2008; Orb, Eisenhauer, \& Wynaden, 2001). Harm may include not only physical but also psychological harm such as stress, distress, losing self-esteem, and discomfort (Bryman, 2008; Houghton, Casey, Shaw, \& Murphy, 2010). For example, during the interview a participant who was sharing her stillbirth experience displayed emotional stress. She could not speak for a while due to the painful memories. I turned the audio recorder off and waited for her to calm down; and advised we could finish the interview on another day. While the qualitative researcher is busy collecting rich and sensitive data (including descriptive details), the participants' privacy, identity, and confidentiality may be exposed without any protections (Houghton et al., 2010; Orb et al., 2001). I understood the responsibility of the researcher and I was and am aware that Koreans who participated in this research shared their private stories and personal life experiences with me as a researcher.

Ethical issues in qualitative research are sometimes unpredictable and these ethical problems may not have simple solutions; however, researchers will be able to cope with these issues if they prepare them early (Batchelor \& Briggs, 1994; Orb et al., 2001). Orb et al. (2001) advise that the researcher's awareness of ethical responsibility and employment of well-established ethical principles will ease the difficulties of ethical issues in a qualitative research. The ethical principles suggested by Chambliss and Schutt (2012) are: "avoid harming research participants"; "obtain 
informed consent"; "avoid deception in research, except in limited circumstances"; and "maintain privacy and confidentiality" (p. 44).

Informed consent means that participants understand the research they are taking part in and the research requirements (Israel \& Hay, 2006). Before beginning the interview, all participants were informed as to the research purpose and the identity of the researcher (Appendix 1, 2; Participant information sheet in Korean and English). In order to protect the participants from potential harms and risks, they were required to sign a consent form prior to commencing the interview (Appendix 3, 4; Participant consent form in Korean and English). To ensure the voluntary participant's privacy and to keep data safe and secure, all participants' identities were kept anonymously. Each participant was assigned a code at the start of the data collection process, and the codes of the participants replaced their names in all notes and transcriptions. All interview data were stripped of identifiers. For example, the name of a Korean GP in Auckland, which was mentioned by a few participants, was replaced by "xxx" in the transcripts. The printed transcripts and the signed participants' consent forms were kept in a locked draw; digital transcripts were kept on a password-protected computer; and audio files were downloaded to a personal password protected computer and deleted from the recorder. Even if ethical principles may not promise ethical research (Orb et al., 2001), bearing them in mind enabled me to be aware of my ethical responsibility. The following was my mantra during the research process: "If it is not right do not do it; if it is not true do not say it"-Marcus Aurelius. This research was reviewed and approved by the Human Ethics Committee of Victoria University in Wellington (Appendix 5).

\subsection{Setting and recruitment}

Participants were recruited from Wellington and Auckland, two major cities in the North Island. Most Korean immigrants (85.6\%) reside on the North Island (Statistics New Zealand, 2014a), and the Auckland region is the most common area for Koreans to live (72.9\%). Recruitment first started in Wellington and moved to Auckland. At the beginning, to find a way to access the Korean community to recruit participants, I sought advice on how to achieve access. I am a Korean but I did not 
have a proper network or sufficient information about Korean immigrants in New Zealand because I was new (I came to New Zealand to undertake this research). The initial information about Koreans and the Korean community in Wellington was gathered from the Korean community newspapers, magazines, and websites. I also emailed the Korean embassy in Wellington to get advice, and visited Korean business places (grocery and gift shops, and restaurants), attended Korean church (Wellington Good Church) and the Korean social club (Kimchi club). The pastor at the Korean church introduced my research to the church members at the service and advertised it in the church bulletin. This was very helpful. Nineteen participants were recruited in Wellington. Recruitment in Auckland went much easier and faster than in Wellington. This was probably due to the larger Korean population (nearly 22,000 people) and the Korean residential clusters in Auckland (Statistics New Zealand, 2014a). I was introduced to two Korean women who were influential and had a broad network in the Korean community. With their help, I met Korean Catholic church members, people who were in a voluntary community service, and visited a senior citizens club (Browns Bay Senior Citizens club). Many Korean immigrants in Auckland not only participated in the research (as participants/interviewees), but voluntarily recruited participants for the interview. Finally, 29 Koreans participated in Auckland.

When I met potential participants, I initially introduced the research and myself to them. If they were interested, I provided the Participant Information Sheet (Appendix 1,2) to help them have a clear understanding of the research, participants' rights, and the type of involvement being asked. However, I tried not to share my opinions or concerns and any extra information related to the issues of the research with the participants. This was to avoid imposing pre-conceived ideas onto the participants; therefore, enabling them to talk about their experiences and thoughts freely at the interview (Elliott \& Higgins, 2012).

\subsection{Sampling methods}

Three different sampling methods were employed; purposive and snowball sampling to recruit the participants, and theoretical sampling to control the data 
collecting process in order to find data relevant to an emerging theory (Glaser \& Holton, 2004). At first, participants were recruited through the purposive sampling technique from the Korean community (such as Korean churches and social clubs) in both Wellington and Auckland. Since purposive sampling (Patton, 2001) recruits the participants who are relevant to the purposes of the research, I recruited Korean immigrants who met the sampling criteria of the research. When the initial participants were recruited by purposive sampling, the snowball sampling technique was adopted together with the purposive sampling method.

"Snowballing" is an appropriate sampling method when recruiting participants who are vulnerable or marginalised within a small pool of populations, and is useful to recruit participants who meet the specific criteria of a research through available social networks (Morgan, 2008). Korean immigrants are a minority ethnic group in New Zealand. Accordingly, the snowball sampling method seemed suitable to recruit Korean participants and actually it worked well for this research. For example, participants were asked to refer or voluntarily referred potential participants for the additional interviews. Their assistance was crucial because the participants, who understood the research (after the interview) and who knew the community, were able to find eligible participants for the research. With this sampling technique the sample size grew efficiently like a rolling snowball.

Although snowball sampling has been adopted by many qualitative researchers, it has some disadvantages (Bryman, 2008; Morgan, 2008). This sampling method works based on participant's personal social networks (e.g., family members, friends, or colleagues). Thus, people referred by the participants are more likely to be from their personal networks and share common characteristics, such as cultural beliefs, perceptions and behaviours. This means, the selection of participants in this sampling method largely relies on the initial contacts (Bryman, 2008). Accordingly, the participants recruited through the snowball sampling are less likely to represent the entire population of the research, and it can be subject to possible sampling bias. As Morgan (2008) recommended, I applied purposive sampling together with snowballing technique, instead of relying only on the snowball 
sampling method. This helped me to maximise diversity in participants and to minimise sampling bias.

Theoretical sampling was applied when the data (collected from the participants who were recruited by purposive and snowball sampling) generated some theoretical ideas. Once theoretical sampling was applied, further recruitment and data collection were directed by theoretical sampling. Theoretical sampling can be referred to as purposive sampling due to its purposive sampling mechanism; however, Jeon (2004) pointed out the distinct differences between the theoretical sampling and the purposive sampling techniques. Purposive sampling selects the participants at the beginning of the research and the selection is determined by the research questions, whereas theoretical sampling is an ongoing sampling method which is determined by data analysed through constant comparison (Glaser \& Holton, 2004; Glaser \& Strauss, 1967). In other words, theoretical sampling responds to the ongoing data analysis throughout the coding process. Therefore, that theoretical sampling can only be adopted when the collected data are analysed and generate theoretical ideas. Once theoretical sampling is adopted, further recruitment and data collection are directed by theoretical sampling according to the analysed data (Charmaz, 2014). Glaser and Strauss (1967) stated, a researcher who adopts theoretical sampling "jointly collects, codes and analyses the data and decides what data to collect next and where to find them, in order to develop the theory as it emerges" (p. 45). Theoretical sampling enables the researcher to achieve apposite data and refines the concepts and categories which were generated from the data; as a result, a conceptualised theory emerges from the data with theoretical sensitivity (Charmaz, 2014; Glaser \& Strauss, 1967).

\subsection{Characteristics of participants}

Forty-eight participants were recruited, 19 from Wellington and 29 from Auckland. Participants were first generation Korean immigrants in New Zealand. Most (90\%) moved to New Zealand between 1992 and 2011, and were residents, permanent residents or citizens of New Zealand $(n=45)$; work permit holders $(n=2)$; or student visa holders $(n=1)$. There were 13 males and 35 females, ranging in age 
from 34 to 86 . Most participants were married and lived with family members, except for three widows and two divorced females. Amongst them, nine participants were full-time workers, six were part-time workers, and 10 were self-employed. The other 23 participants were made up of 13 home-makers, eight retirees, and two unemployed due to their physical conditions at that time. Participants' healthcare experiences in New Zealand and in Korea varied from each other. The demographic characteristics of the participants is provided in Appendix 7.

\subsection{Data collection}

In grounded theory, "The initial decisions for theoretical collection of data are based only on general sociological perspectives and on a general subject or problem area" (Glaser \& Strauss, 1967, p. 45). Researchers collect data with an open mind and with minimal preconceptions in order to achieve theory naturally and directly from the participants' perspectives without forcing or contaminating. In this research, the main data were collected from the 48 participants in face-to-face indepth interviews. The other types of data gathered and prepared were memos (e.g., field notes, audio recorded memos, and visualised memos), a research journal, informal conversations, literature review, and advertisements from Korean magazines and websites (related to the health-seeking behaviour of Korean immigrants in New Zealand).

As the iterative process of concurrent data collection and analysis is typical of grounded theory (Glaser \& Strauss, 1967), the data collection process in this research was led by the preceding findings of the data analysis. In keeping with the theoretical sampling method, I repeatedly collected the data to find data relevant to emerging theoretical ideas. The data were analysed as soon as possible following each interview so that further interviews were able to be guided by the data themselves. This method helped to develop theoretical ideas and saturate the emerging theory from the data, and narrowed it down as the research progressed (Dastjerdi, Olson, \& Ogilvie, 2012; Jones \& Alony, 2011). Data were collected from April to November 2014. In Figure 1 the concurrent, iterative and integrative theory building procedure carried out in this research is demonstrated and explained. 


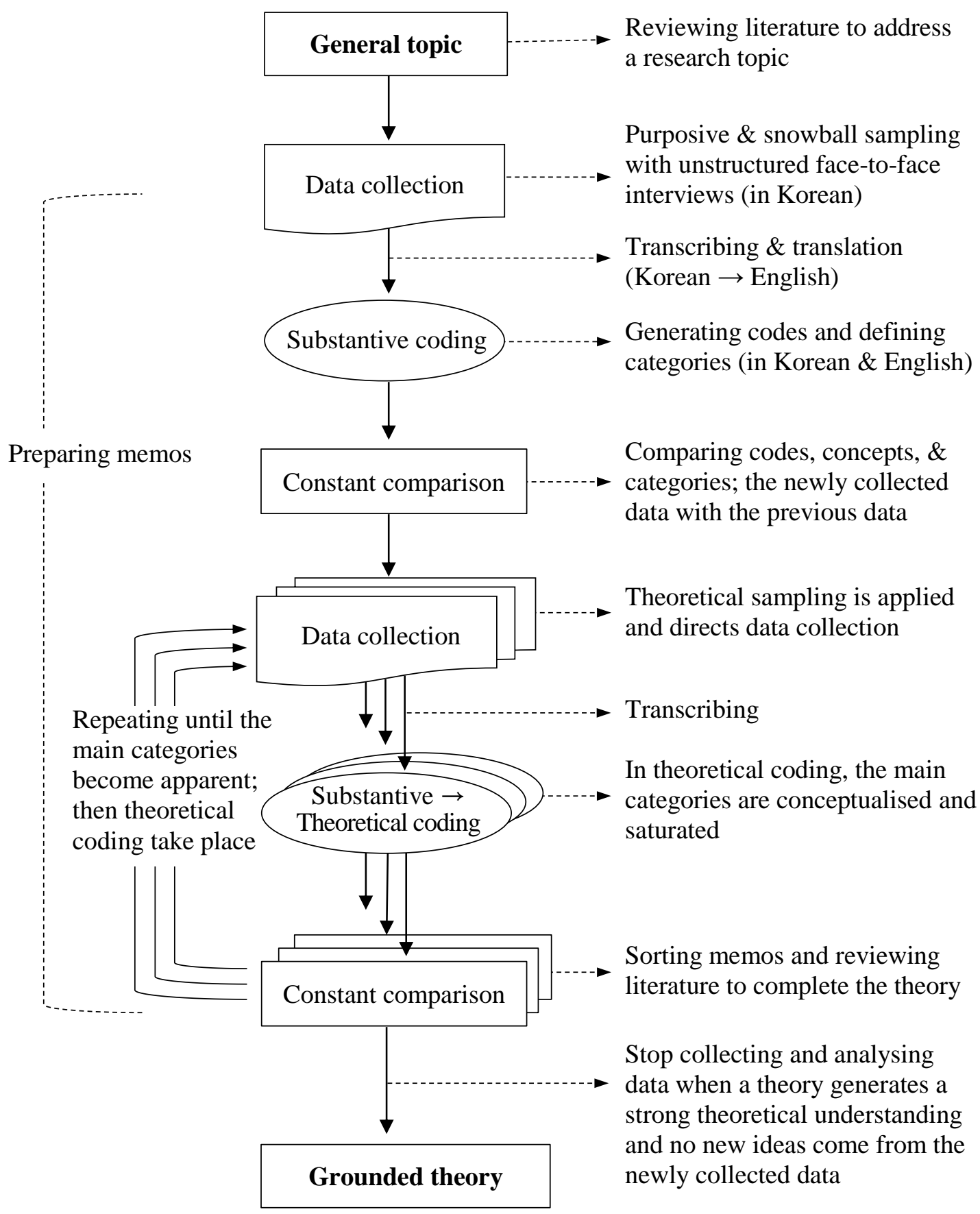

Figure 1. Theory building procedure

\subsubsection{Interviews}

Interviews are the most common method of data collection in grounded theory like qualitative studies (Charmaz, 2014). They are particularly useful when participant observation is not an option or when the participants' experiences are 
largely unknown (Merriam, 1997). While individual interviews are considered as an appropriate method to collect in-depth and detailed personal information on sensitive issues, group interviews allow a wider range of information and render collective opinions and attitudes which explain groups of people's understanding of social context (DiCicco-Bloom \& Crabtree, 2006). To enhance data richness, I employed both individual and group interviews to collect data. Participants were given the option of being interviewed individually or in groups, or both. The place and time for each interview was decided together by myself and the participant(s). The interviews took place in participants' homes, business places, offices, schools, and sometimes restaurants or coffee shops. I tried to choose the place where participants could talk comfortably with minimal disturbance. All interviews were conducted in Korean (as participants preferred speaking Korean), and the interviews were audio-recorded with the participants' permission. Interviews lasted 30 to 120 minutes depending on the participant's responses and situations. There were 23 individual interviews, seven two-person interviews ( $\mathrm{n}=14$ [two people attended twice]), and four group interviews $(\mathrm{n}=14$ [one person attended twice]).

In the beginning of the research, as Charmaz's (2014) constructivist grounded theory was the chosen version of grounded theory for the research, I prepared an interview guide (Appendix 6). Having an interview guide with written introductory questions and a set of topical questions helped me feel less anxious about interviewing participants. The questions included in the interview guide were about healthcare experiences, perceptions, strategies, and the contributing factors in their healthcare decision-making. However, when the interview commenced, I realised that participants' healthcare experiences and their thoughts were far broader and more complex than the interview questions (even though I intended to prepare the interview guide questions broad enough and tried not to point out the specific issues of the research), and some of the initial questions were also too abstract to answer (e.g., How do you perceive your health risk and needs? or What kind of healthcare do you seek?). Furthermore, the interviews themselves generated the key words or topics to focus on, so I was able to continue the following interviews with these topics (e.g., GPs are not specialists, regular comprehensive medical check-ups in 
Korea, or kind and friendly medical staff). The interview guide was not as useful as expected and I began to question its necessity in grounded theory research.

Glaser (2012) stated, "If the data is garnered through an interview guide that forces and feeds interviewee responses then it is constructed to a degree by interviewer imposed interactive bias" (p. 30). Although doing grounded theory focuses on uncertainties and the uncertainties diminish as the research progresses, if one wants to understand the main concerns of the research participants, using a prepared interview guide may not be appropriate, especially at the beginning of the research. I decided not to use the interview guide and started the interview with broad questions; for example, "Tell me about your health", or "Tell me about your healthcare experiences". I applied the theoretical sampling method to guide the interviews and to develop theoretical ideas that emerged from the participants. As a result, the interview focus was narrowed down with theoretical sampling. How the theoretical sampling method guided the interviews is explained in the latter Section 4.9.2 Constant comparison and theoretical sampling. The interview method in this research shifted from semi-structured to open-ended unstructured, and the interview guide which was used for the early interviews was replaced by the theoretical sampling. This occurred before I changed the version of grounded theory for this research from Charmaz's constructivist to Glaser's classic grounded theory. During the research, my role as a researcher and interviewer also moved from a passionate participant (Denzin \& Lincoln, 2005) to a passive listener (Glaser, 2012).

\subsubsection{Memos and other sources of data}

Regarding data Glaser stated, "All is data" (Glaser, 2001, p. 145). Grounded theory allows for all types of data to be used to discover a pattern grounded in the phenomenon studied (Glaser, 2012; Glaser \& Holton, 2004). Particularly, memos play a major role in grounded theory analysis. Researchers capture their analytical thoughts and ideas as memos, and these memos reflect what the researcher knows and how he/she knows it (Lempert, 2010). Memos in grounded theory are not simply to remember or store researcher's thoughts and ideas, rather to develop theoretical ideas from the data and to analyse them (Holton, 2008). Using memos facilitates the 
data analysis to move from a descriptive level to a theoretical level (Holton, 2010). Memoing is a continual process in grounded theory as Holton stated, researchers in grounded theory utilise memos throughout the research.

There were different types of memos used during the data collection and analysis in this research: hand-writing memos, voice recording memos, diagrams, mind-maps, and post-it memos; as well as a research journal and field notes (as memos are entries in a research journal or field notes). I tried to memo as quickly as possible to catch my analytic ideas at that moment. I wrote memos freely without any worries about grammar, spelling, or tidiness, and used either Korean or English, or both; because I prepared them for myself. My observations of the participants (such as facial expressions, voice tones, body language, and interactions within the groups) including my personal thoughts were recorded in the form of field notes during or immediately after the interview. When interviewing participants face-to-face, I realised that keeping eye contact with them was as important as taking memos and taking memos could interrupt the participants' stories. Particularly, when the participants were talking about very sensitive and private issues. I made notes as simple and short as possible during the interviews, and when the interview was finished, I quickly moved to another place to memo what I could not memo at the interview. Table 1 and 2 are examples of memos after interview.

Table 1. Memo A: After interview

\begin{tabular}{l}
\hline Code_T0X \\
She views her and her family healthcare experiences in New Zealand based on the \\
previous healthcare experiences in Korea. Her perceptions are rooted in her experiences. \\
She displayed frustration, dissatisfaction, and disappointment with respect to healthcare \\
experienced in New Zealand. She had enough healthcare experiences in Korea and has a \\
clear idea about healthcare that she wants. Her healthcare expectation might be too high and \\
unrealistic. However, she expects healthcare as a mother of a child and as a wife of a man. \\
Her family health is her responsibility. Her husband was generally quiet during the \\
interview. His healthcare experience in Korea was almost zero, so he does not have things \\
to compare to healthcare experiences in New Zealand. His perception towards the New \\
Zealand health services was quite positive even though he did not express strongly it. \\
Expectations are directly connected to experiences (what you know) and it seems that if the \\
expectation is too high it is more likely to be dissatisfied.
\end{tabular}




\section{Table 2. Memo B: After interview}

\begin{tabular}{l}
\hline Code_INOX \\
\hline \multicolumn{1}{|c|}{ Immigration, moving to a foreign country with family, is a huge life decision. } \\
Health determines quality of life, means everything. It should be one of the top priorities in \\
our lives. But he (not only him, others also) did not check the New Zealand healthcare \\
system before he immigrated to New Zealand. How and why? Furthermore, his main reason \\
for immigration was his deteriorated health. He chose New Zealand and moved here because \\
he wanted to live healthy and spend more time with his family. BUT, he did not try to know \\
the New Zealand healthcare system. It is hard for me to understand. I need to check more \\
about it....He left Korea 20 years ago. He was pushed by the busy and competitive lifestyle \\
in Korea. I can see that immigrants who have strong push factors seem to adapt better into \\
the host society.
\end{tabular}

I used a voice recorder and made voice notes when I needed to capture my thoughts quickly or when hand-writing was not possible. I wrote a research journal as the researcher and as an individual with a background, personal life and personal characteristics. Much of what I wrote was not important or not relevant to the research, but it revealed my preconceived ideas and potential prejudices and made me more aware of possible researcher's bias to the data during the research.

Once theoretical ideas and concepts started to emerge from the data and as I began to understand the meanings of the data, my thoughts and ideas also started to activate. I often utilised visualised memos (e.g., diagrams, charts, mind maps, and post-it memos) to stimulate and facilitate my analytical thinking. Lempert (2010) stated that visualising thoughts and ideas helps to organise ideas and stimulates their analytical thinking, and visualised memos enable the researcher to look at the data with analytical distance. I drew diagrams, charts and mind maps as a strategy to capture and illustrate my incomplete thoughts and blurry ideas. I was actively involved with analytic thinking through the activity of visualising itself, and it helped me refine rough thoughts and to find connections between scattered ideas while drawing memos. Adopting visualised memos guided the data analysis and theorising process, and it resulted in generating a theory at conceptual levels. Several examples of diagrams made in this research are located in Appendix 8-10. These sequential diagrams (A, B, C, D, E) show the theory shaping process in this research. 
Table 3. Memo C: After informal conversation

Code_INOX
When I met her recently, she was happy to share with me her new healthcare
experience at the public hospital. She seemed to have lost weight compared to the time we
met in May 2014. For the past few months, she had abdominal pain and visited her GP a
few times due to the symptom. However, her GP said that she was normal. Finally, she
presented with high fever, intense sweating, vomiting, and severe abdominal pain. She went
to the ED and the hospital kept her for three days for observation. This story is exactly the
same as another participants' healthcare experience. How and why does it happen quite
often???...She took blood tests and ultrasound scan at the hospital. Gallstones and high
cholesterol levels were found and they were the main causes of the problem. It was the first
time she was hospitalised in her life. Despite her fear (due to her symptoms and the first
hospitalising experience in a foreign country), she was impressed by the caring medical
staff and their sincerity. It changed her healthcare perceptions (except the GP service); she
started to view the New Zealand health services positively (which was negative before).
Now follow-up is ongoing. This updated healthcare experience of participant gave me
another chance to confirm the theory that emerged from this research.

I also had informal conversations with some of my participants. I had several chances to meet the participants and they shared the healthcare experiences they had had since the interview and their opinions of these. Their experiences and opinions were used as memos and assisted the data analysis providing another form of meaningful data (Table 3). In addition, articles and commercial advertisements from Korean websites and magazines and newspapers in the Korean communities in New Zealand with respect to Koreans' healthcare and their health-seeking behaviours were collected. Specifically, Korean comprehensive medical check-up advertisements, which targeted Korean immigrants' health-seeking behaviour in New Zealand, assisted to develop one of the categories in the research.

\subsection{Transcribing and translation}

When each interview was finished I transcribed the audio-recorded interviews as soon as possible, so that the interview data could be moved to the analysis stage. After listening to the recorded interviews repeatedly I had a better understanding of them; however, transcribing was a lengthy process and needed patience. I tried to focus on the main concerns of Korean immigrants in healthcare while listening to the participants' voices and prepared memos to figure it out. Once an interview was 
transcribed in Korean, I immediately translated it into English. In qualitative research the data are words rather than numbers, and qualitative researchers, who translate one language into another language, interpret the meaning of the words from the participant's point of view (van Nes, Abma, Jonsson, \& Deeg, 2010). At the early stage of the coding process, my concern was how to translate precisely and correctly; as I thought, it would be the best way to prevent the participants' words from losing their own meaning. However, grounded theory, particularly classic grounded theory focuses on conceptualisation in the theory generation beyond the description (Glaser $\&$ Holton, 2004). Soon I realised that the translation in grounded theory studies is more than literal translation (accurate word-for-word translation).

Concepts are the names given to a pattern in data and the names which capture the best fitted meaning have conceptual power (Glaser, 2002). Naming concepts and categories with a best-fitted meaning within two different languages was the tough part of this research. In order to select an appropriate English word that has the right meanings for the Korean word, I had to carefully examine the meanings of words in English and in Korean as well. Translating in grounded theory was not simply delivering meanings of the words, rather constructing meanings between two languages (Tarozzi, 2011). For example, there was a common expression amongst the research participants termed "nurturing disease (병을 키우다)". It could also be translated as "raising/bringing up disease". It indicated deterioration of disease resulting from failure to take appropriate treatment before it deteriorated, which was a sarcastic expression. This expression implies feelings of "blame", "regret" or "disappointment" because it was preventable. Although "nurturing disease" is a very common expression in Korea and amongst Koreans, it needed extra explanation in order to deliver the actual meanings of the participants behind the words.

Language carries culture (e.g., customs, values, and feelings); that is, there would be no absolute conceptual equivalence across languages (Temple \& Young, 2004). I had to examine the words of the participants (Korean) and search for the words in English with the best-fitted meanings even if they were not literally the same in meaning. When the appropriate English words were found to replace the 
names of concepts and categories in Korean, I queried these English names with English native speakers around me to get their understandings of the words and their opinions. I discussed the meanings with them and confirmed the suitability of the words to minimise the gap between two languages. When the concepts and categories were developed as data analysis progressed, and they were named in English, I decided not to translate all transcripts (cover-to-cover), translating only those parts that were necessary. Translating entire transcripts from Korean to English benefited in developing concepts and categories at the early stage of the coding process (open coding); however, it was not worthwhile after the concepts and categories were established and named due to its time-consuming nature.

Conducting grounded theory in English, as a non-native English speaker, was an obvious challenge. Bilingual researcher Tarozzi (2011), who sees translating as an act of intercultural mediation, claims that the benefits of doing translation in grounded theory outweigh its difficulty because doing translating itself provides powerful analytic resources. I have to agree that I was able to grab the grounded meanings of the words while I was struggling between two languages, and it became a foundation for the conceptual theory development.

\subsection{Data analysis}

Researchers in grounded theory, "look for patterns in data, any kind of data, name (conceptualize) those patterns, identify relationships between the conceptualised patterns, and write them into a theory" (Simmons, 2011, pp. 6-7). According to Charmaz (2014), coding allows the researcher to view data in a refined way. However, researchers are often challenged by their preconceptions while they interact with the data, and if data are forced into preconceived codes and categories it is considered poor research practice. Attaining an in-depth knowledge of participants and understanding of the studied phenomenon helps the researcher to avoid imposing his/her preconceived ideas into the research (Charmaz, 2014). The classic grounded theory coding process was adopted to analyse the data in this research. I personally believe in emergence of theory as Glaser insisted; "trust that emergence will occur and it does" (Glaser, 1992, pp. 3-4). A theory that has emerged from the data (by 
constant comparative analysis with theoretical sampling) will fit and work, if it does not fit and work the theory is forced (Glaser \& Strauss, 1967).

I decided to code the data manually because this research aimed to understand Koreans' healthcare experiences and concerns, and adopted classic grounded theory to render a theoretical explanation through a conceptual theory emerged from the participants' perspectives. During the interviews, I heard participants' voices, observed their facial and body expressions, and felt empathy; and when I coded the data I remembered them. The coding was based on my understandings of the participants rather than the literal meanings of the participant's words. Holton and Walsh (2016) stated that the researcher in grounded theory must engage in coding systematically with constant comparison and generate conceptual ideas through memoing, even though they are time consuming. Accordingly, coding by automatic software is not recommended in classic grounded theory. The concern raised regarding the use of automatic data analysis software in grounded theory studies is the loss of analytic creativity in the theory generating process and this will result in losing analytic power of grounded theory, even if it is convenient, quick, and easy to use. The coding process in classic grounded theory is divided into two stages: substantive coding (includes open and selective coding processes) and theoretical coding (Holton, 2010).

\subsubsection{Substantive coding: Open coding and selective coding}

Coding aims to generate codes and find relevance to develop theoretical concepts and categories, and integrate them into a theory (Glaser, 1978; Strauss \& Corbin, 1998). The coding process in this research began when the first interview was transcribed and translated from Korean to English. I listened to the audiorecorded interviews and read the transcripts repeatedly to get a sense of direction for analysis and to find a latent pattern grounded in Koreans' health-seeking behaviours in New Zealand. In open coding, transcripts were analysed word-by-word and lineby-line, and the words or phrases of the participants were used as substantive codes; 
this method is referred to as "in vivo" 28 coding. According to Charmaz (2014), in vivo codes capture the meanings of the participants' perceptions and actions, and help researchers to stay close to the data to avoid imposing their personal feelings or impressions on the data. At the open coding stage, I generated as many codes as possible as Glaser and Strauss (1967) suggested, as diverse properties of the category developed from the various data helped to fill the gaps in categories and in theory. The more interviews conducted, the more codes were generated from these interviews. I tried to name concepts and categories with progressive gerunds to emphasise the participants' actions and the movements in data in order to understand their behaviour through their actions. For example, "trying to be healthy", "encountering GP service", "rejecting the New Zealand health services", "negotiating healthcare options" and "trusting and attuning". The gerund form (-ing) of concepts and categories provide strong feelings on actions, and it helps researchers to concentrate on data and its process (Charmaz, 2014; Glaser, 1978).

I was not sure how to carry out data analysis with two different languages at the beginning of the research. However, I decided to use both languages for the data coding and analysis until the main categories were clarified and they had fixed English names. At first, I coded the Korean transcripts and generated codes in Korean, and the Korean codes were found in the English transcripts simultaneously or translated to English directly. The codes, concepts and categories that emerged were named in both Korean and English; the examples of codes in two different languages are "GPs are not specialists (지피는 전문의가 아니야)"; "I was told (내가 들었는데)"; "Listens well (잘 들어 줘)". The problem of using two different languages in one code was the existing gap between languages including the cultural gap between Korea and New Zealand. As indicated previously, searching for English words that can deliver the same meanings as Korean words was challenging. However, carrying out coding with two different languages (rather than choosing one of the languages) offered chances to examine the meanings of participants' words in-

${ }^{28}$ The word "in vivo" comes from the Latin "in that which is alive"(Saldana, 2009). The codes in "in vivo" coding are actual words or terms of the participants (Bluff, 2005). Qualitative research and grounded theory use this coding method as one of the initial coding methods (Saldana, 2009). In vivo coding also refers to "literal coding" and "verbatim coding". 
depth. Particularly, the Korean labels of the codes and concepts helped me to remember the participants' voices as the participants spoke in Korean at the interviews and as many codes and concepts came directly from their words. I felt that Korean codes were easier to manage than English codes as Korean is my mother tongue.

In selective coding, the codes, concepts, and categories were constantly compared one to another, and the data newly collected compared with the previous data. Theoretical sampling with constant comparative analysis guided the subsequent sampling of participants and interviews, and the emerging concepts and categories were continuously examined and supported by the data collected by theoretical sampling (Holton, 2010). As a result, the concepts and categories were refined and achieved saturation. During the selective coding some concepts and categories disappeared or merged with others, whereas some concepts and categories were enhanced and saturated by emerging codes through the constant comparative analysis and theoretical sampling. For example, the early data showed that Koreans' English proficiency was one of the major factors affecting their health service utilisation in New Zealand. "Language difficulty" was placed as a theme under one of the categories; however, it disappeared as more interviews proceeded. According to the data revealed later, their language proficiency did not affect their health service utilisation as much as they thought; even if it might influence their competence or confidence in health service utilisation. Language was not a major problem in their healthcare because Koreans in this research usually could get help from one of the family members (who could communicate in English). They knew through their healthcare experiences that doctors and nurses at the hospital speak (English) according to the patient's levels of English to make sure their patients understand; otherwise, they can use the interpreter service.

Another early category was labelled as "Natural healing" which was brought directly from the words of Korean immigrants. As more data were collected, it was found that the meaning of natural healing was not exactly the original meaning of natural healing; it was closer to "self-care" or "self-management". Natural healing, which can be referred to as "natural medicine" or "complementary and alternative 
medicine (CAM)", prefers to use natural remedies rather than science-based medicine (Calabrese, 2012). It focuses on self-care management and supports the body's own healing process within the natural environment. Korean immigrants were largely used to visiting hospitals and taking injections for even trivial illnesses. For them, the meaning of natural healing was taking care of themselves by taking a rest and without seeing doctors even if they took medication from pharmacies. The category, "Natural healing" even though it came from the participants' own words, was changed to "Self-care and self-awareness" 29 . During the selective coding, the relevant data were collated into categories; as a result, their subsequent data collection and coding became less, and the selected categories became saturated (Glaser \& Holton, 2004; Holton, 2010). The codes, concepts, and categories developed in the research are attached in Appendix 11.

\subsubsection{Constant comparison and theoretical sampling}

Constant comparative analysis is a typical data analysis in grounded theory (Glaser \& Holton, 2004; Glaser \& Strauss, 1967). The iterative process of data collection, transcription, and data analysis occurred concurrently with the constant comparative analysis method. There are three levels of comparison proposed in grounded theory (Glaser \& Holton, 2004; Glaser \& Strauss, 1967). The first level of comparison is between each incident in order to find the uniformity or similarity amongst data and their different conditions. At the second level, concepts are compared to more incidents in order to generate more theoretical properties; as a result, concepts are saturated and conceptualised. The third level of comparison is between concepts and it aims to achieve theoretical saturation. Consequently, conceptualised concepts and categories are integrated into the emerging theory.

During the coding process, the emerged theoretical ideas from the data were constantly compared with the newly collected data by theoretical sampling; the concepts and properties were formed by similar kinds of codes, and these concepts and properties were compared with the newly collected data from the new

\footnotetext{
${ }^{29}$ See the Section 5.7.1 Healthcare in New Zealand is ideal: Self-care and self-awareness (p. $151)$.
} 
interviews; and they were enhanced, expended, modified, or disappeared. The concepts and categories that emerged through the constant comparative analysis were compared again with various analytic memos that I prepared during the data collection and analysis process. This comparison with analytic memos cleared and defined the connections between the concepts and categories and saturated them further. When they were integrated and theorised in a theory, then I compared the theory with the relevant literature to contextualise the theory within the current knowledge. Constant comparative analysis was adopted throughout the coding process to generate a theory that fitted into the studied phenomenon.

The derived emerging concepts and categories through constant comparative analysis directed the following samplings and informed the next interviews, which refers to theoretical sampling (Holton, 2010; Jones \& Alony, 2011). Theoretical sampling responds to constant comparative analysis and controls the data collection to collect the data that fit into the emerging theory (Glaser \& Holton, 2004). Charmaz's research (2006) on people with chronic illness showed how data collection is shaped by theoretical sampling. Her initial data analysis found that people with chronic illness often measure their health according to their level of daily activities. This finding offered a subsequent interview with focused questions to explore the deeper meanings of these measures. Similarly, the early data analysis in this research showed that Korean immigrants' healthcare assessments were influenced by the functional aspects of health services rather than their technical aspects. Korean immigrants' health-seeking behaviours were more likely to be changed when they were affected by the attitudes of the medical staff and/or the ambience of health services. In order to find a latent pattern which could explain this behaviour, further interviews and data collection were narrowed down to the relationship between the type of healthcare participants experienced and its influences on their health-seeking behaviours.

\subsubsection{Theoretical coding}

Once numerous substantive codes and categories are identified and categorised, the main categories become apparent, then theoretical coding takes place 
(Holton, 2010). While substantive coding (open and selective coding) works with fractured data and groups them according to their attributes, theoretical coding concentrates on saturated categories and examines the relationship between those categories (Jones \& Alony, 2011). Researchers in theoretical coding sort memos into emergent categories and employ literature to cross-reference to theorise main categories (Holton, 2010; Pleizier, 2010). Sorting memos helps the researcher to figure out the theoretical relation of memos to concepts and concepts to categories; as a result, the final conceptual categories are revealed with theoretical saturation, and a theory emerges from these central categories. Then the researcher analyses the relevant literature in order to complete the theory (Holton, 2010). The final product of the theoretical coding is a conceptual theory that renders a theoretical explanation of the main concerns of the participants from their perspectives (Jones \& Alony, 2011).

During the theoretical coding process, Koreans health-seeking behaviours were defined and classified into the four main categories: "rejecting", "attuning", "attuning but negotiating", and "rejecting but negotiating". They were modified, extended, and saturated from the earlier categories such as "beginning/unknown", "separating", and "attuning" as seen in the diagram memos (Appendix 9, 10). Sorting memos through constant comparative analysis helped me to clarify theoretical connections between concepts and categories; as a result, the more relevant categories were developed and completed and the less relevant categories were disappeared. For example, in the theoretical coding process, the category "beginning" or "unknown" was dropped because they were theoretically less meaningful to the emerging theory compared to other categories. Meanwhile, the categories "attuning but negotiating" and "rejecting but negotiating" were developed and refined because they explained the specific health-seeking behaviour of Korean immigrants that could not be covered by the previous categories.

When the theory took its shape after sorting memos, I went back to the literature to contextualise the emerged theory to existing knowledge (Strauss \& Corbin, 1998). I reviewed the extant literature to make sure the findings of the research fit into the current existing knowledge in the studied area. Particularly, 
Berry's (1997, 2003, 2005) model of acculturation was influential even though his model covers the acculturation as a whole, while this theory deals with acculturation in the healthcare domain which is one of the many domains of acculturation. Berry's understanding of the acculturation process from a psychologist's perspectives ${ }^{30}$ helped me to develop a deeper insight into the healthcare acculturation process of Korean immigrants in New Zealand. The research findings were compared and analysed with the relevant literature and integrated into the theory. I discussed and introduced the relevant literature with the findings of the research in the following chapter titled Findings. I stopped further data collection and analysis when the emergent theory generated a consistent and strong theoretical understanding, and no new idea emerged from the newly collected data.

The coding process in classic grounded theory emphasises theoretical integration; theoretical codes conceptualise the connections between codes, between categories, and between codes and categories (Glaser \& Strauss, 1967). The additional data, which were collected by the theoretical relevance, were continuously collected, coded, and added to the emerging categories until the categories were fully saturated and conceptualised. This coding process was efficient and worked well even though it was time-consuming. It was like a puzzle; interviews generated pieces of the puzzle, and the gaps in the puzzle were filled by the new pieces collected through constant comparative analysis with theoretical sampling which helps to find suited pieces. Accordingly, many substantive codes were generated during the coding process to fill the gaps between codes, and categories were constantly compared and grouped into similar categories until the main categories were defined. The codes were classified and grouped together and labelled to develop conceptual main categories (Appendix 11).

\subsection{Managing rigour in theory generation}

My priority in managing rigour in this research was to make sure the theory was generated directly from the data and not from existing hypotheses or my own

\footnotetext{
30 The detailed description of Berry's model of acculturation is provided in the Section 2.4 Acculturation (p. 30).
} 
preconceived ideas. The constant self-awareness of the implicit actions and meanings of the researcher in the research process is a significant component in grounded theory, because the research findings can be influenced by the researcher's biases and prejudices without noticing (Gasson, 2004). To avoid distortion by my personal biases during the data analysis, I utilised various types of memos and a research journal. While I recorded analytic thoughts and ideas, my personal biases and prejudices were also recorded there. These records, consequently, reminded me of the potential biases and prejudices that I had, and helped me to be aware of them during the research. Engaging a critical self-awareness required me to be constantly self-critical and to scrutinise each research process conducted. I believe that this minimised my influences on data and increased the rigour of the research.

Classic grounded theory assesses its quality via the criteria of fit, work, relevance, and modifiability (Glaser, 1978). I utilised constant comparative analysis with theoretical sampling to ensure theoretical sensitivity in the theory generating process in order to satisfy the criterion of "fit" into the studied phenomenon. To meet the criteria of "work" and "relevance", this research analysed Korean immigrants" healthcare adapting process and clarified their different adopting pathways (with their reasons and consequences) and different healthcare approaches. Koreans' health-seeking behaviours were conceptualised into the Healthcare Acculturation Theory, and this substantive theory explains their behaviours and healthcare concerns in New Zealand. When a theory is able to adopt new ideas from new data, the theory meets the criteria of "modifiability"; and if the theory is modifiable, the theory will fit and work, and be relevant (Flint \& Johnson, 2010). The Healthcare Acculturation Theory was developed to understand Koreans' health-seeking behaviours in New Zealand. Therefore, in order to apply this theory to other immigrant groups, it needs to be tested. This means, this theory is not a final outcome; it can be modified and expanded further when relevant new data appears.

While qualitative methodologies concentrate on multiple levels of confirmation (e.g., triangulation) to achieve rigour of the research (Mertens, 1998), grounded theory focuses on the research method itself; the rigour in grounded theory is assured by constant comparative analysis and theoretical sampling during the 
theory generation process (Elliott \& Lazenbatt, 2005). The constant comparison and theoretical sampling methods in grounded theory provided the research with a rigorous theory development procedure. It is believed that if a theory is rigorously generated from the data, then the theory would be applicable to explain the issues of the studied area. In the research, the ongoing concurrent data collection and analysis were guided by the constant comparison and theoretical sampling methods throughout the research process, and utilising these methods together with memoing helped to preserve theoretical sensitivity in theory generation and keep rigour of the research. Grounded theory methodology required time, thought, patience, diligence, and commitment. To achieve rigour in grounded theory research, the researcher has to work at it; there are no shortcuts (Corbin \& Strauss, 2008).

\subsection{Summary}

The chapter provided detailed descriptions of the theory generating process implemented in this research. It included how participants were recruited and how data were collected and analysed. Purposive and snowball sampling methods were employed to recruit participants with theoretical sampling guidance. Forty-eight participants were recruited from Wellington and Auckland, and the main data were collected from unstructured face-to-face individual, two-person, and group interviews. I discussed my role as a researcher during the interviews and the reasons for the change in type of interview from the semi-structured to the open-ended unstructured. The recorded interviews were transcribed and translated from Korean to English. The various types of data were collected and prepared during the research, and demonstrated with examples.

Coding adopted Glaser's classic grounded theory coding process (substantive coding and theoretical coding). I demonstrated the use of grounded theory in a single research involving two different languages; particularly, during the coding process. As classic grounded theory focuses on theory emergence, the iterative, congruent, and integrative data collection and analysis process was guided by constant comparative analysis with theoretical sampling, and this process continued until the 
data generated a conceptual theory with a strong theoretical understanding. This chapter ended by providing a discussion of how I managed rigour in theory generation. The findings of the research together with the substantive theory that emerged from this research are presented and discussed in light of the literature in the following chapter. 


\section{Chapter 5. Findings}

\subsection{Introduction}

The chapter provides Korean immigrants' transition pathways in the process of healthcare acculturation in New Zealand. In the healthcare adapting process what Koreans' main concerns in healthcare were and how they resolved them are explored and discussed in detail. In keeping with Glaser's classic grounded theory Korean immigrants' experiences and their health-seeking behaviours are conceptualised and theorised. The substantive theory that emerged from this research, namely the Healthcare Acculturation Theory, is introduced in this chapter. The healthcare adapting process of Korean immigrants within the New Zealand healthcare system is illustrated in Figure 2. The theory building process is provided step-by-step with a series of figures (Figure 2- Figure 6) and theoretical explanations.

There were four healthcare positions revealed as main categories of the Healthcare Acculturation Theory: "rejecting", "attuning", "attuning but negotiating", and "rejecting but negotiating". Korean immigrants adopted one of the four positions as their healthcare approach within the New Zealand healthcare system based on their healthcare experiences and their perceptions of the host health services. The chosen position reflected Koreans' specific perceptions, attitudes, and behaviours toward the New Zealand health services, and influenced their health service utilisation and health-seeking behaviours as well. During the healthcare adapting process, their health-seeking behaviours were reconstructed by adapting to the New Zealand healthcare system. Accordingly, their healthcare positions were modified or transitioned. The factors contributing to these behavioural transitions and the consequences of these changes are also explained by the Healthcare Acculturation Theory.

In grounded theory, literature is integrated into the theory as another form of data to complete the theory. The research findings are presented with a discussion based on the literature review conducted after data analysis. 

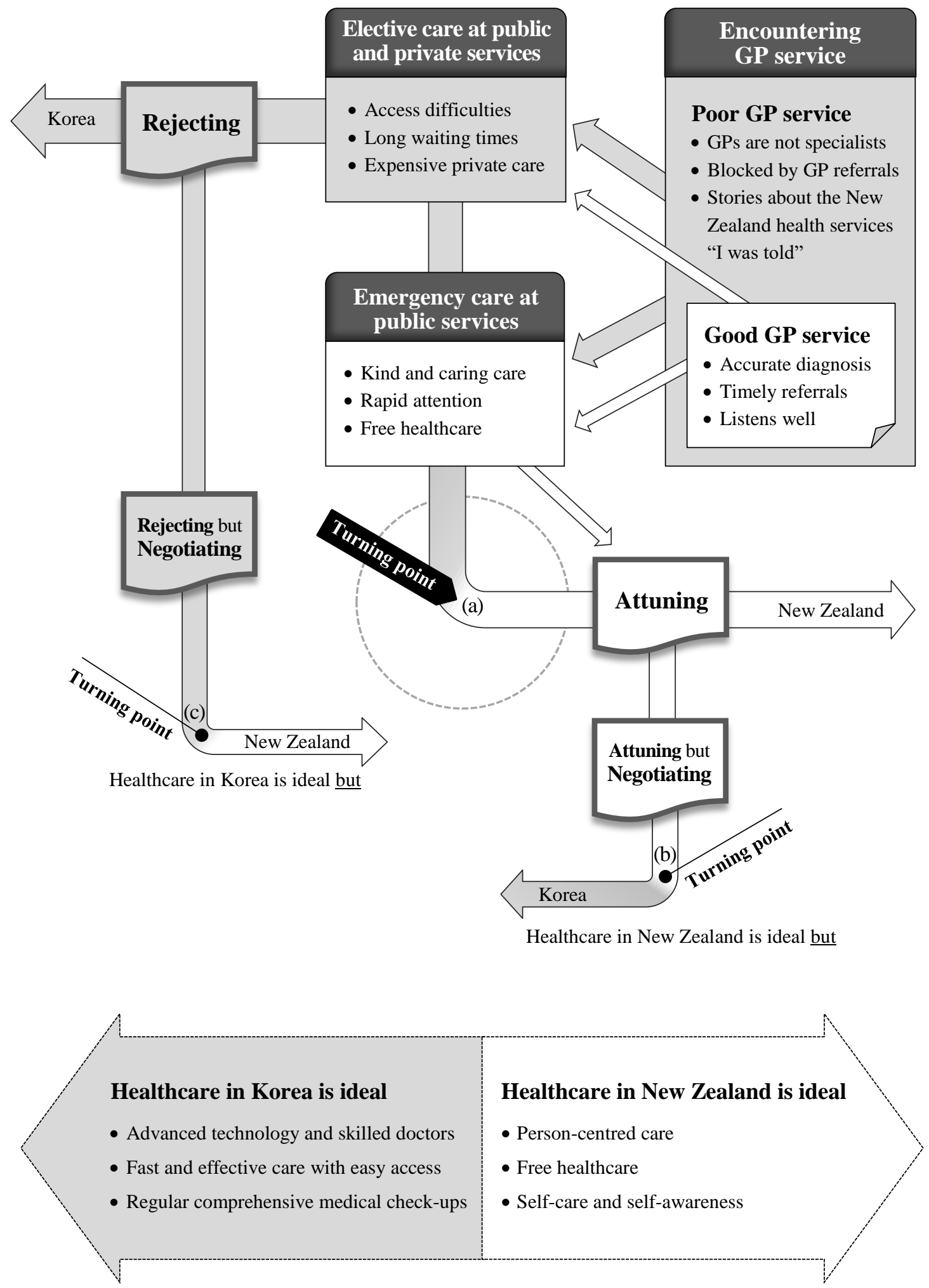

Figure 2. Healthcare Acculturation Theory 


\subsection{Beginning}

\subsubsection{Purpose of immigration}

Koreans came to New Zealand looking for a better quality of life rather than for economic reasons (Epstein, 2006; Kim \& Yoon, 2003; Song, 2013; Yoon \& Yim, 1997). They chose New Zealand for a relaxed lifestyle, clean and green nature, and a less stressful education system in an English environment, rather than the competitive education system, stressful working conditions, and crowded and polluted living environment in Korea (Chang et al., 2006; Kim \& Yoon, 2003). They sought the values and qualities in life that they could not have in Korea, and they found them in New Zealand. According to O'Reilly and Benson (2009), relatively affluent immigrants who "escape from somewhere and something" (p. 3) and look for something that fulfils their desires in order to have a better quality of life, like Korean immigrants in New Zealand, are termed lifestyle immigrants.

The purpose of immigration for the participants in this research was similar to those of Koreans in the research literature. They were attracted to the relaxed lifestyle, the quiet and uncrowded environment and beautiful nature in New Zealand, and an English school system for their children's education. Common words used when they described New Zealand included: “clean”, "green”, "beautiful”, “calm”, "peaceful", and "relaxed". For example, a woman with 14 years residence portrayed her first impression of New Zealand, "I saw a paradise. Wherever I went, it was a dream world with green, green grasslands" (G01a:25). The environmental beauty of New Zealand often captivated Koreans and some of them saw it as a "paradise" (Kim $\&$ Yoon, 2003). They were "pulled"31 by New Zealand, but they were also "pushed" by some factors in Korea such as stressful lifestyle, highly competitive working environment and education system, and political corruption.

A man in his 40s moved to New Zealand in 1999 after experiencing the financial crisis in Korea in 1997. Companies in Korea had a severe downsizing and voluntary retirements were encouraged at that time. "I believed in 'jobs for life' but it

${ }^{31}$ The Push-Pull Model (Lee, 1966) explains migration based on the push (minus) and the pull (plus) factors of both origin and destination. See the Section 2.3.1 Theories of migration initiation (p. 25). 
collapsed in a moment. I was full of fear for my future” (T06a:16). Another male participant revealed his strong feelings toward Korea while explaining their reasons for immigration. "I left Korea because I dislike Korea....There was so much political corruption. I was upset and disappointed, so I left Korea without looking backfor a second" (IN06:311). Other reasons for immigration reported by participants included marriage (five females had married Koreans in New Zealand); family reunification (three senior participants moved to New Zealand to stay with their children who were already New Zealand citizens or residents), and job opportunity (a husband of one participant had a job offer).

Lee's (1966) Push-Pull Model proposes that migration might occur as a consequence of comparison when migrants calculate the push (minus) and the pull (plus) factors of both origin and destination, and when the pull factors at the destination outweigh that of the origin, then migration is likely to occur. In this regard, the immigration decision to New Zealand seemed an inevitable outcome for the participants who were pulled by factors in New Zealand (the relaxed and better lifestyle, an English environment for their children's education, and peaceful and natural environment), and pushed by factors in Korea (the competitive working environment and education system, and political corruption). Their reasons for immigration were largely similar but varied in details according to their personal situations. In their immigration decision, the plus and minus factors in both countries were taken into account, and generally considered rationally but sometimes emotionally. A few participants' immigration decisions were largely due to the pull factors in New Zealand rather than the push factors in Korea; for example, they came to New Zealand for their own reasons such as marriage, family reunification and a job offer. In addition, none of the participants reported that his/her immigration decision was influenced by the New Zealand healthcare system. They were not even interested in health or the healthcare system when they came to New Zealand.

\subsubsection{When I came here}

Participants' ages ranged from 26 to 60 years when they came to New Zealand. Most classified themselves as "young" and "healthy". Some participants 
specifically articulated, "I came to New Zealand when I was in my early 30s. I was healthy because I was young" (IN10:3); "I was not interested in health because I was young" (IN05:109). During the early days of immigration, they rarely utilised health services and many of them did not even register with the GP (general practitioner) service: "We didn't get sick at all during the first three years, so we didn't have a GP" (T04a:78); "I didn't need to see a GP because I was well" (IN21:30). This low health service utilisation of Korean immigrants is reported in the New Zealand Health Survey ${ }^{32}$ 2013/14 (Ministry of Health, 2014b). Asian ethnic groups, such as Koreans, utilised the primary health services less than all other ethnic groups in New Zealand. They were found with comparatively low levels of healthrelated risk factors, including smoking, and have less incidence of some chronic conditions, such as asthma, arthritis and chronic pain. That is, their lower health service utilisation compared to other ethnic groups was most likely due to their reduced healthcare needs as a consequence of their overall better health status. This phenomenon is commonly explained by the "healthy immigrant effect" (Kennedy et al., 2006; McDonald \& Kennedy, 2004; Mehta, 2012). Immigrants, more precisely the first generation of immigrants (foreign-born), are generally healthier than the native-born citizens, and that is mainly due to the criteria of the immigration policy, which are more favourable to young and healthy individuals.

There was a lack of knowledge of the New Zealand healthcare system amongst the participants, particularly during their early days in New Zealand. Some participants acknowledged, "I learned about the GP service one year after I came here" (IN07:37). "I did not know what a GP was at that time....My kid was sick and we just went there because it had a cross sign like a hospital" (G01a:63). Participants often complained about the difficulties in getting health service information and their limited English proficiency was one of the main reasons.

I made a huge decision because I loved my husband so much. I am young. I am in my mid-30s. I thought everything was going to be alright if I tried hard. But every aspect of this culture is so different, such as the "roundabout" and driving direction. I feel helpless when I imagine

${ }^{32}$ This longitudinal survey (New Zealand Health Survey) focuses on health and wellbeing of New Zealanders, and provides annual information about smoking, nutrition, access to healthcare and oral health of adults and children (Ministry of Health, 2014b). 
the situation if I got sick. Just thinking about hospitals in New Zealand and their confusing systems makes me so frustrated. These things are not explained in their brochures [in hospitals]. Besides, they are all in English. (IN19:126)

The participant quoted above had lived in New Zealand for three years following her marriage to a Korean man in New Zealand. She realised that her parents-in-law, who had lived more than 20 years in New Zealand, also did not understand the New Zealand healthcare system. She decided to study it, but learning a new culture and the new healthcare system in a foreign language was a huge challenge and stressful for her. She looked for and found health service information in Korean from the New Zealand based Korean communities (such as the Korean Community Association and the studying abroad agents' websites for Korean students in New Zealand) and her Korean friends. She also emailed and asked for help from a Korean who worked at a healthcare facility in Auckland, and got a brochure, which explained the healthcare system and services of New Zealand in Korean. "It contained quite broad information about the New Zealand healthcare system, but it wasn't clear enough. I was more confused after reading it" (IN19:137). She was not satisfied with the information because she felt that it was superficial. Being an immigrant without knowing the available health services with language difficulties made the immigrant Koreans feel insecure and anxious. Another participant who had been a resident for 14 years stated, "For people like us without English skills, there is no way to get information. No one explains it to us", (G01a:450). Koreans' seeking health service information and learning about the healthcare system in New Zealand were hampered by their language difficulties rather than their gender, age, or duration of stay.

Research on immigrants' health indicated that health service utilisation of immigrants, particularly for newcomers, was restricted by their information or knowledge level of health services in the host country; and their language skills crucially determined obtaining the new health services information of these immigrants (Choi, 2013b; Harari, Davis, \& Heisler, 2008; Scheppers, van Dongen, Dekker, Geertzen, \& Dekker, 2006). There was a lack of information and knowledge of healthcare and health services in New Zealand amongst the participants 
(particularly, in their early days of immigration when they were young and healthy), and it was heavily influenced by their language barrier. However, according to the outcomes of this research, the participants' low health service utilisation in their early stages of immigration was due to their age (young) and health status (healthy) rather than their low level of knowledge.

\subsection{Healthcare acculturation}

Most Korean immigrants in this research encountered challenges in adapting to a new healthcare system in New Zealand. They experienced changes in their health-seeking behaviours during the process of healthcare adaptation as a result of interaction with the New Zealand health services. Healthcare acculturation is an adapting process within the new host country's healthcare system. As "healthcare" is one of the many domains of acculturation (Salant \& Lauderdale, 2003), healthcare acculturation is a part of acculturation as a whole. While Korean immigrants interacted with the New Zealand health services, their healthcare perceptions, attitudes and behaviours toward the host healthcare system were formed based on their healthcare experiences with the New Zealand services and their previous healthcare experiences in Korea. During the process of healthcare acculturation, their perceptions and behaviours were reconstructed or modified as their healthcare experiences were continuous. The process of healthcare acculturation was reflected in the Koreans' healthcare perceptions and attitudes and their health-seeking behaviours; also, their immigrant lives were influenced by their levels of healthcare acculturation as well.

Korean immigrants in the research experienced various health issues, such as pregnancy and childbirth (including miscarriage and stillbirth), pulmonary oedema ${ }^{33}$, diabetes, cancers, colds, and accident injuries. However, each participant's healthcare experiences in New Zealand varied. They had different levels of health service utilisation due to their health status and different lengths of stay, and they utilised different types of services according to their age, illnesses, and the presence

\footnotetext{
${ }^{33}$ Pulmonary oedema is a condition caused by excess fluid accumulation in the lungs, and it leads to impaired gas exchange and may cause respiratory failure (Brunner, 2015).
} 
or absence of insurance. The health services they experienced in New Zealand were always compared to health services in Korea. Their perceptions were formed and their "healthcare positions" ${ }^{34}$ were taken based on these evaluations. When participants' existing perceptions and healthcare positions were challenged by new service utilisation, transitions in their perceptions and healthcare positions took place in the process of healthcare acculturation.

\subsection{Encountering GP service}

Most participants' healthcare experiences in New Zealand started when they encountered the GP service. Many of them had never experienced a GP service and did not even know what a general practitioner (GP) was before they came to New Zealand. When they visited a GP for the first time, they realised that GPs were not specialists, unlike doctors in Korea, and they began to learn the differences between the health service in New Zealand and Korea. The healthcare system in Korea, which is largely privatised (approximately 94\%), is delivered by specialised doctors, not GPs; thus, the role of GPs were not commonly understood by Koreans (Kwon et al., 2015). There is no gatekeeping role in primary healthcare in Korea; accordingly, patients have access to specialist care without any restrictions.

As patients tend to use their prior experiences as a baseline when it comes to healthcare expectations, immigrants' healthcare perceptions are not free of their preconceived ideas of the healthcare system in their home countries (Lien et al., 2008; Son, 2013). One of the most distinct factors affecting the healthcare adapting process of immigrants is the gap between the home and host countries' healthcare systems (Choi, 2013a; Leduc \& Proulx, 2004; Lee, Kearns, et al., 2010). In this perspective, Korean immigrants in New Zealand who came from a very different healthcare system are likely to have more adapting problems. Figure 3 presents Korean immigrants' perceptions toward the GP service and the factors affecting these perceptions.

\footnotetext{
34 "Healthcare positions" reflects immigrants' healthcare approaches within the host healthcare system. It implies perceptions, attitudes, and behaviours of immigrants toward the host healthcare system including their levels of involvement in health service utilisation.
} 


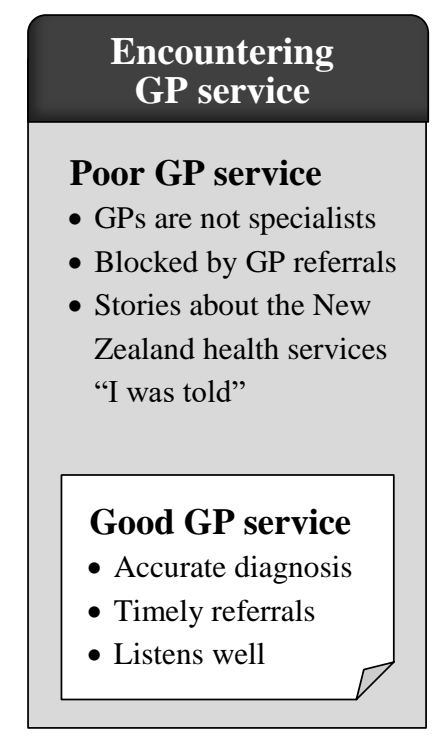

\section{Figure 3. Healthcare Acculturation Theory: Encountering GP service}

\subsubsection{Poor GP service}

After encountering the unfamiliar GP service, participants were largely dissatisfied with the service (Figure 3). Their disappointments were mainly due to the GPs' ineffective treatments, including their overly optimistic responses toward health symptoms. They believed this occurred because GPs were not specialists.

Participants felt frustrated with the GP service when they were confronted by the referral system. Participants, who were used to having a high level of access to specialist care in Korea, had to book and wait to see a GP in order to access specialist care and further medical examinations. Nonetheless, it did not simply guarantee a referral. They became anxious about their health and healthcare in New Zealand, and they tended to adhere to negative news and stories about the New Zealand health services around them; "I was told". They viewed the GP service as poor compared to the health services in Korea which are mainly delivered by specialists. Their healthcare needs and expectations were largely unmet within the GP service, and these experiences left participants with a strong negative impression towards the healthcare system in New Zealand. 


\section{GPs are not specialists}

When the participants visited a GP for the first time, they were surprised at the capability and scope of GP practices, which involved diagnosis, treatment of broad health problems, medical screenings, minor surgeries, and referrals (Paterson, 2015). A woman with 15 years residence expressed her curiosity towards the GP practice, "A GP does all, examines every part of the body!...How does one doctor screen cervical cancer and examine throat and ears?" (IN01:125). Participants' first response after utilising the GP service was, "GPs are not specialists". They were often confused with the GP's response towards their health symptoms: "My GP often says 'it will be alright. It is nothing'. For him almost everything is nothing", (T02a:73); '“You are alright!', 'very good!', and 'it is normal'. He [GP] says that all the time and it actually makes me feel better....A little while later, however, the symptoms came back again" (IN08:289). A woman in her 30s who had been resident in New Zealand for seven years reported:

The GPs' instructions are weird. Simply "it is normal" or "endure". When I went to hospitals in Korea, I used to get medication for my illness. So it helped me to get quick relief. In here, just Panadol! ${ }^{35}$ No one [GP] prescribes the medication that we used to get in Korea. That is why I am reluctant to see a GP here. (T03b:154)

When the participants visited GPs, they expected healthcare attention for their symptoms from their GPs. They visited a GP because they recognised their symptoms as an illness, but their GPs diagnosed them as normal (absence of disease), so medical treatments were not necessary. The participants' perceptions of illness did not match that of the GPs; therefore, their healthcare needs and expectations were unmet. Individuals' perceptions of illness are influenced by social and cultural factors; how that society interprets the symptoms and how physicians in society respond to it (Boruchovitch \& Mednick, 2002; Conrad \& Barker, 2010). Koreans who participated in the research perceived and interpreted their symptoms according to the perceptions of illness that they had in Korea. They expected their GP to

\footnotetext{
${ }^{35}$ Panadol is a medication used to relieve pain and reduce fever from colds and sore throats, headaches, toothaches, backaches, and reactions to vaccinations (shots) (American Society of HealthSystem Pharmacists, 2014). Panadol is also as known as Acetaminophen in the USA and Tylenol in Korea.
} 
provide treatment that they used to have with their doctors in Korea. That is, fast and effective health services which commonly provided injections and strong medication. They were used to having access to a wide range of prescriptions in Korea, regardless of whether they wanted or needed these or not.

Participants often questioned the prescriptions given by the GP. "The GP prescribed Panadol, That's all” (IN21:572), “Always [a GP says], 'Take Panadol,”, (T04a:525). "Panadol for the shoulder pain and Panadol for a cold" (IN16:923). The healthcare experiences with the GP service led the participants to be more favourable to specialist care and made them to believe that the GP's treatment was ineffective. Participants felt that their healthcare needs, both physical and psychological (emotional), were disregarded based on the GPs common responses, "You are alright!", "You are normal!", or "Take Panadol!" Although the reasons for seeing a GP amongst the participants were varied, their perceptions of the GP service were similar; GPs' treatments were ineffective because GPs were not specialised physicians. One participant in his 50s had to visit a GP three times with the same symptoms.

He [GP] checked me and said I am alright. I could not eat well because of the pain and lost 4-5kg at that time. I was not alright. I told the GP [upset and raised voice], "You said exactly the same thing before. I want to have a gastro-photograph to check my symptoms. I have insurance. I need a test even if you keep saying to me I am okay". (T04:320)

The GP suggested he take a blood tests first, and he (GP) quickly organised another test (ultra sound) when he saw the result of the blood tests. The ultra sound showed stones in his gall bladder and the GP referred him to the emergency department (ED) immediately. "He used to diagnose me superficially and said I am alright. I cannot trust him anymore" (T04:515). A woman who had carpal tunnel syndrome ${ }^{36}$ spoke about her GP. She felt that she did not receive proper treatment

\footnotetext{
${ }^{36}$ Carpal tunnel syndrome (CTS) is the most common peripheral nerve problem resulting from compression of the median nerve at the wrist caused by increased pressure in the carpal tunnel (Bland, 2007). The symptoms include numbness, tingling of the thumb and radial fingers, aching wrist, and clumsiness. It is usually a chronic condition and females aged between 40 and 60 years are at highest risk.
} 
from the GP but the instruction "Do not use the hand and be careful!" (T05a:189). However, she had to wait until the GP referred her to a specialist and she did not know the exact cause of the pain until saw a specialist. She said, "In here [New Zealand], it took me three months to figure out what the problem was" (T05a:206). The healthcare experiences with the GP service, especially the ineffective treatments and poor outcomes the participants received, resulted in a low level of satisfaction, and undermined the relationship between the participants and GPs. Some participants who were disappointed with the GP service articulated, "I don't trust GPs" (IN05:186; IN06:162; IN21:769; T04a:575). A man who had a sinus infection reported:

GPs can only ask about symptoms. They do not have medical equipment to diagnose inside the nose, so their treatment is simply based on listening to the patients. That is why I do not trust GPs, besides it cost 40-50 dollars to see a GP each time. "Be careful and take Panadol!" I did not pay to hear that. (IN05:182)

Another woman also stated, "I felt frustrated when a GP could not diagnose my symptom" (T02a:818). The GP consultation fee was frequently criticised by the participants. They found that the fee was too high relative to the ineffective outcome, and this concern determined their further utilisation of the GP service: "I hesitated to see a GP even though I felt ill, and it was due to the cost....It is not worthwhile because they are general practitioners not specialists" (T04a:309); "I want to see a specialist directly when I am ill. Then I will be able to have trust" (IN01:148). Most participants firmly articulated their healthcare preferences for specialist care after experiencing the GP service. They assessed the healthcare received from their GPs based on the outcomes; however, these assessments were inevitably influenced by their previous healthcare experiences in Korea. Simultaneously, their perceptions toward health services in Korea, which were formed in Korea, were re-evaluated according to the healthcare they experienced with the GP service in New Zealand. "If it [illness] happened in Korea, it would be solved by visiting an ENT specialist just once" (T03b:122). The more unsatisfactory healthcare experiences with the GP service generated the more positive perceptions of the Korean healthcare system 
amongst the participants. Consequently, participants considered the health services in Korea as an ideal standard of healthcare for them.

\section{Blocked by GP referrals}

Patients' access to secondary care is regulated by the referral system in New Zealand, and GPs, whose role is gatekeeping, make decisions for patients whether a specialist referral is necessary or not (Cumming et al., 2014). Therefore, patients without a GP referral cannot approach secondary and tertiary levels of healthcare except the ED and after hours service. GPs' gatekeeping in primary care aims to limit unnecessary access to secondary level care, and to provide better quality care to the patients when they need it (Foot, Naylor, \& Imison, 2010; Forrest et al., 2000; Piterman \& Koritsas, 2005). On the contrary, there is no gatekeeping function in primary healthcare in Korea, so patients have the freedom to choose a specialist and have direct access to their (specialist) practice without referrals (Chun et al., 2009; Ock et al., 2014).

In Korea, we used to visit specialists freely. Most doctors in Korea were specialists even the doctors at the small clinics. Whenever I needed them, I could see them. But I have to see a GP first here. To get medication or to see a specialist I have to visit a GP first. It bothers me, bothers me a lot. (G03c:101)

From the participants' point of view, those who were used to freely visiting specialists in Korea, seeing a GP first in order to access secondary care was a barrier; further, GPs' first assessments did not simply guarantee a referral. Some participants complained of the difficulties in getting a GP referral: "Once a GP says you don't need that and that's it!" (IN02:244); "I have to explain and persuade [a GP] in order to get a referral. 'I think I need this. I want. Could you do it for me? Please.' like that" (IN20:444). Many participants' healthcare needs for specialist based care, which they were used to, did not reach the threshold of the GPs referral. One participant whose husband had gastric bleeding and ulcers was disappointed when the GP prescribed "Zantac" for him. "I think he [GP] should have organised a medical examination for my husband if the symptom was so serious....My distrust of GPs started from that time" (IN07:144). There was strong desire for specialist care 
amongst the participants that stemmed from the disappointed healthcare experiences with the GP service; however, their desire was blocked by the GPs' non-referrals. A male participant in his 40 s had a chronic migraine for 15 years.

In this country, it is not possible to get a CT [Computerised Tomography] or MRI [Magnetic resonance imaging] without the GP's permission. Even though I asked for these tests due to my headache, it was not possible. I like to have these tests even though it cost me $\$ 1000$. I am willing to pay for these tests but still the GP's referral is mandatory. So there is no way....My GP always says, "It is just migraine”. (T06a:102)

The GP considered the symptom of the participant as not serious enough for specialist care or further medical examination, whilst the participant viewed his symptoms as underestimated by the GP. Conflicts took place between the participants and GPs due to the disagreement on the severity of the symptom and a lack of referral for secondary care. Patients' health service evaluation might be different to that of the physicians who provided the health service; however, the degree of understanding between the patients and healthcare providers determined patients' satisfaction and their relationship as well (Alrashdi, 2012; Petersen, 1988). Participants felt that their opinion with respect to their healthcare needs and expectations were disregarded within the New Zealand healthcare system. They did not know what to do and where to go when refused referrals by their GPs. Some said: "I have to wait until my symptom deteriorated to see a specialist" (IN11:117); "In fact, it was better that my symptom deteriorated because it gave me access to the ED service" (T01:869). This was the question raised by one female participant: "Why do I have to see a GP first? Why does this system work this way?" (IN19:267).

\section{Stories about the New Zealand health services "I was told"}

After experiencing the GP service over time, participants gradually understood that they would not get the same attention and treatment that they used to get in Korea, and they became anxious about their health and healthcare in New Zealand. Patients with unsatisfactory healthcare experiences are more likely to be affected by the negative stories and news around health services (Migge \& Gilmartin, 
2011). A number of Korean immigrants in this research, who had adverse experiences with the GP service, also tended to be somewhat easily swayed by negative news and stories about the New Zealand health services around them. "I was told, one Korean couldn't wake up from the general anaesthesia of a surgery” (IN05:55). "Someone I know had a hard time after colonoscopy because it left a hole in the colon" (IN15:454). "I was told, here, I have to wait very long to get healthcare. And people die before receiving treatment" (T04a:121). "I could often hear from people around me, 'someone was ill but a GP missed the diagnosis,", (T05a:215). "I was told, I should not trust even if a GP says 'it is alright", (IN01:139).

Participants' anxiety doubled with these negative stories and information. They were overly worried about a misdiagnosis that is likely to be caused by a superficial examination by their GPs who were not specialists and delayed treatment as a result of the misdiagnosis. Consequently, anxiety was rooted in the participants, and they were reluctant to utilise the GP service. One woman who had nephritis ${ }^{37}$ spoke about the reason for the delayed GP visit. "Because I thought the GP would say 'you are normal'....How could I know it was nephritis?" (T03b:343). A female participant who had herpes simplex (according to her self-diagnosis) avoided seeing a GP. She said, "GPs did not seem to know skin problems well...and their prescriptions would be Panadol, Panadol for everything” (T02a:69). Participants, who were disappointed and suspicious about the GP service, lowered their expectations and declined to use the service in order to avoid their perceived risks. However, it resulted in them putting their health at further risk such as delaying diagnosis and undertaking treatment based on self-diagnosis.

Participants' healthcare experiences were largely involved with the GP service; many of them did not have experience with other health services in New Zealand, such as ED service and secondary and tertiary levels of care. They perceived the New Zealand health services based on their healthcare experiences

\footnotetext{
${ }^{37}$ Nephritis is the inflammation of the filtering units of the kidneys, which causes impaired kidney function (Kidney Health New Zealand, 2015). Nephritis can be due to a variety of causes, including kidney disease, autoimmune disease, and infection.
} 
with the GP service. Accordingly, the participants who perceived the GP service as poor also viewed all New Zealand health services as poor. These negative perceptions directly affected their attitudes, behaviours, and healthcare decisions until new healthcare experiences challenged these perceptions.

\subsubsection{Good GP service}

While most participants perceived the GP service as poor, a small number of participants reported good healthcare experiences with the GP service. Their satisfactory experiences with the GP service were mainly due to their GP's accurate and quick diagnosis, and timely referrals (IN09:105; IN10:32, 61; G11a:61). For example, a married couple who took care of their aged parents highly appreciated their GP. "Above all, his diagnosis is very accurate and quick" (IN09:105). They believed that the GP, who diagnosed his parents' illnesses correctly (father's pulmonary oedema and mother's aneurysm) and referred them immediately to specialists, saved their parents' lives. Three older participants were also satisfied with their GP. They attended the same GP and agreed with each other, "He diagnoses well and listens to patients well. He makes patients feel comfortable" (G11b:92). They indicated affirmative perceptions of the New Zealand healthcare system due to the good GP service they experienced.

There were also some good statements about the GP service from a few participants, regardless of their perceptions of the GP service whether they were negative, positive, or neutral. One participant said, "The good thing of the GP service is record keeping. My GP knows even my parents' medical history" (IN02:205). A woman who had the same GP for 20 years reported, "He provides check-ups regularly even when I visited him for a cold. I feel comfortable [secure] with him." (G02a:436). Her GP provided or suggested medical check-ups on a regular basis such as blood tests and cancer screenings that she and her husband needed, and it made her feel secure. However, she still preferred the health services in Korea. Another participant who perceived GPs' healthcare as ineffective and preferred to have specialist care, spoke about her GP. 
I heard about other GPs who are not nice and speak fast even if the patient was not an English person....But my family GP is different. She explains in a kind and stable manner. She takes good care of us.

(T03b:138)

The good GP service experiences of some participants were not as influential as the poor GP service experiences. They were positive experiences but somewhat tentative. These experiences could not shape their healthcare perceptions, and could not change their existing perceptions of the GP service or the New Zealand health services. During the utilisation of the GP service Korean immigrants had formed their perceptions of the New Zealand healthcare system, and when these perceptions became strong enough to influence their healthcare decisions and direct their healthseeking behaviours, their healthcare positions were identified as their approach to healthcare within the New Zealand healthcare system.

\subsection{Four healthcare positions: Attuning, rejecting, attuning but negotiating, and rejecting but negotiating}

Participants' health-seeking behaviours generally fell into one of the four healthcare positions at any one point in time. Four healthcare positions, which were revealed as main categories of the Healthcare Acculturation Theory, were "rejecting", "attuning", "attuning but negotiating", and "rejecting but negotiating". Participants chose one of four positions as their healthcare approach based on their healthcare experiences and perceptions in New Zealand. The chosen position indicated participant's certain perceptions, attitudes, and behaviours toward the New Zealand healthcare system including their levels of involvement in health service utilisation in New Zealand and in Korea. Therefore, once a specific position was held, their health-seeking behaviours and healthcare decisions were directed by the said position. As their healthcare experiences were ongoing, their chosen positions were not end-outcomes in themselves. When their existing healthcare perceptions and health-seeking behaviours were challenged by new healthcare experiences, and when the participants responded to these new experiences, their healthcare positions shifted to other positions or reinforced themselves along with the latest healthcare 
experiences, and these changes informed the Healthcare Acculturation Theory. There was a small number of participants whose attitudes and behaviours did not fit into these four categories. Their healthcare positions were not formed yet because they had insufficient healthcare experiences due to their good health status and/or short length of stay.

\subsection{Rejecting: GP service and elective care at public and private services}

Rejecting was a position whereby the immigrants abandoned and disregarded the New Zealand health services due to unsatisfactory experiences within the New Zealand healthcare system. Figure 4 portrays the rejecting healthcare position of Korean immigrants including their reasons for rejecting and its consequences. During the healthcare adapting process, participants took rejecting as their initial healthcare position after experiencing the GP service. They were disappointed with the service due to the GPs who were not specialists and difficulties obtaining

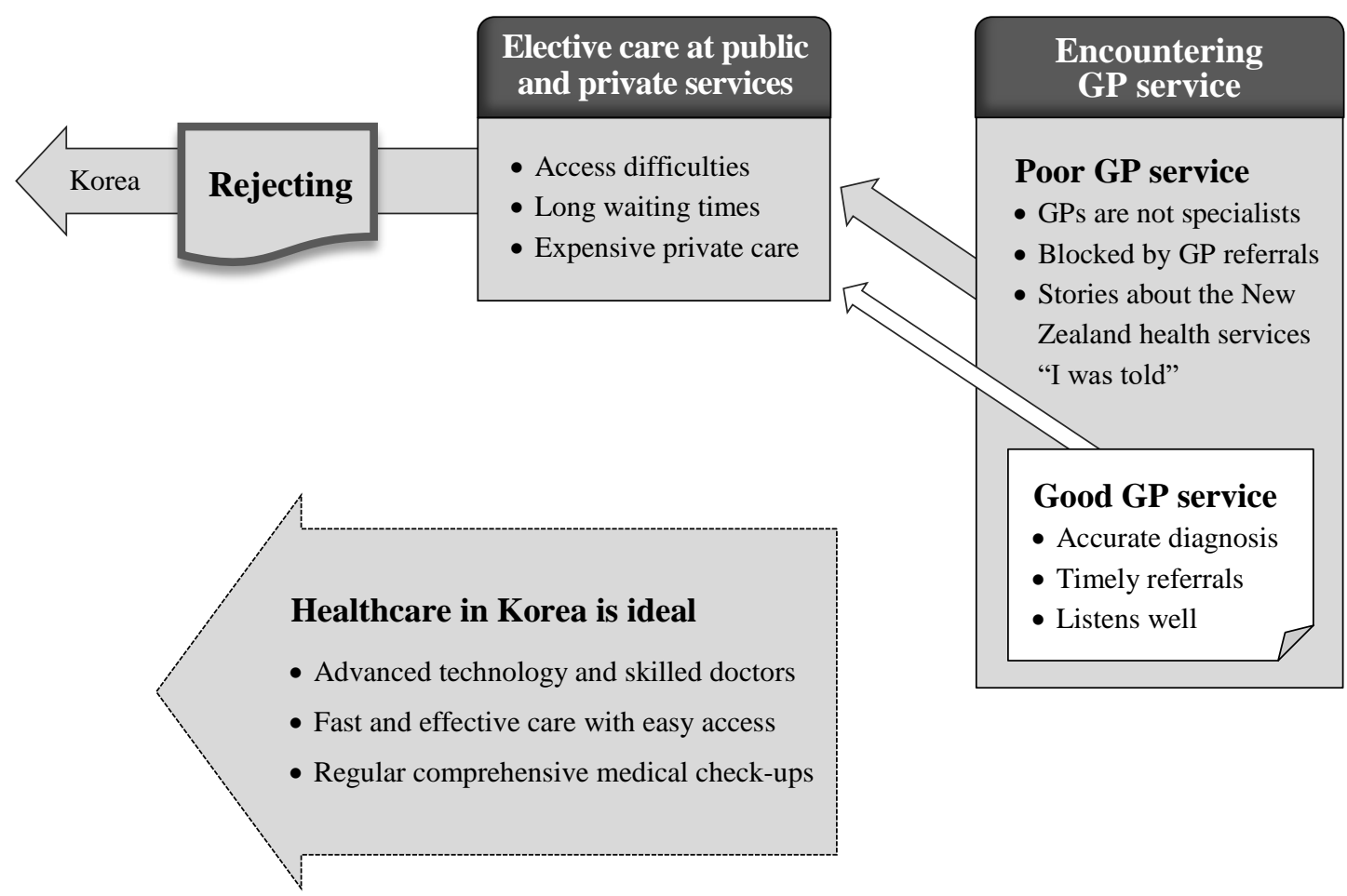

Figure 4. Healthcare Acculturation Theory: Rejecting 
specialist referrals. They were easily influenced by negative stories about the New Zealand health services including the GP service. They perceived the GP service as poor and took the rejecting position. When the participants were confronted with elective care at public and private services, the rejecting position was taken or reinforced. Their healthcare experiences were negative towards these services because of access difficulties and long waiting times for public care (ED service and secondary and tertiary levels care) and the high cost of private care. Their healthcare perceptions were disaffected by these aspects of the services. There were two different groups of participants who made the rejecting decision at this stage. One was the participants, who already took rejecting as their initial healthcare position resulting from previous poor GP service experiences. After experiencing elective care at public and/or private services, their chosen position of rejecting became firmer and more pronounced than before. Another group was the participants who chose rejecting as their initial position due to these healthcare experiences (elective care at public and/or private services), even though some of them had good GP service experiences.

Participants in this research were used to freely utilising health services with various healthcare options in Korea. When they encountered the absence of freedom and restricted options within the New Zealand healthcare system they became more cognisant of the high level of access to health services that they had had in Korea (Andreou, 2013). A man who had a chronic illness (sinus infection) reported, " $M y$ GP said 'I can't refer you to a specialist'... my chronic illness deteriorated for the last 10 years because I couldn't access a specialist in this country" (IN05:26). According to the literature, if the immigrants had no prior experience with the GP service, their healthcare needs and expectations were largely unmet within the GP service in the host country (O'Donnell et al., 2007; 2008). The gatekeeper role of the GP was not easy to understand by the immigrants, who were used to direct access to the specialist care, and they tended to underestimate GPs because they were not specialised doctors. Immigrants expect health services that they were used to in their home countries. This means, Korean immigrants in New Zealand who came from a very different healthcare system are more likely to have more adapting difficulties (Lipson et al., 2003). 
Within the New Zealand healthcare system, participants felt powerless and without agency in terms of access to health services of their choice. Losing the power to control health services was more than healthcare itself, it was losing ownership of their own health, and it left them feeling fearful about risks to their health (Breyer, 2013). New Zealand based healthcare experiences were largely adverse and/or restricted; consequently, participants' perceptions of the New Zealand health services were negative, and they took a rejecting position based on these perceptions.

Participants were disappointed with the New Zealand health services and these experiences turned them away from it. They viewed the health services in Korea as better than those in New Zealand. They believed the healthcare standards in Korea as their ideal standard in healthcare: advanced technology and skilled doctors; fast and effective healthcare with easy access; and regular comprehensive medical check-ups as preventive healthcare. They tried to minimise using the health services in New Zealand, and maximise its utilisation in Korea.

Although they favoured the health services in Korea, their preferences were often confined by time and the cost of travelling to Korea. "I can't visit Korea often, so have to take care of health myself" (IN05:206). They looked for alternatives to avoid the New Zealand health services, and to prepare for the access problems in the New Zealand health system as well. Many of them practiced healthy lifestyles to maintain and improve their health, such as healthy diet, regular exercise, and taking proper rest. They also kept and used Korean medication to manage their minor health symptoms themselves, and took health supplements (such as omega 3-6-9, pro-polis, and multi-vitamins) to prevent disease and keep healthy. Some of them purchased private insurance to have easy access to hospital.

There were a small number of participants who tried oriental therapy (e.g., acupuncture) as alternatives in New Zealand, but they were not satisfied with that. Two of them stated, "I cannot trust oriental doctors in New Zealand" (T04a:1190; T06a:102). The reason they gave was that the quality of oriental medicine in New Zealand was not as good as in Korea. Cupping therapy ${ }^{38}$ was used by a participant

\footnotetext{
${ }^{38}$ Cupping therapy is a form of alternative oriental medicine in which cups are placed on the skin to create suction in order to stimulate the blood flow (El-Wakil, 2011).
} 
who expressed dissatisfaction with acupuncture and chiropractic treatment in New Zealand. "We [he and his wife] learned cupping therapy because there was no other way....The cupping equipment that my mother sent us is really useful because we can take care of ourselves" (IN05:82). He and his wife learned it from his uncle who was an oriental medicine doctor in Korea. In addition, participants with a rejecting position tended to watch Korean Television health channels to get information, and tended to search the Korean websites for information about their health symptoms and treatments. "I always search the internet for my symptoms before meeting a GP....If it can't solve the problem I call a hospital in Korea" (IN16:986).

\subsubsection{Healthcare in Korea is ideal}

Participants were pessimistic about the New Zealand health services when they experienced unmet healthcare needs and expectations within the New Zealand healthcare system. As a result, they chose the rejecting position as their healthcare approach in New Zealand. They came to believe that Korean health services were better and reconsidered health services in Korea as ideal healthcare for them. They strongly favoured the health services in Korea with its advanced technology and skilled doctors, fast and effective healthcare with easy access, and affordable and regular comprehensive medical check-ups.

\section{Advanced technology and skilled doctors}

Participants who took the rejecting position viewed doctors in Korea as more experienced and better skilled than doctors in New Zealand, and valued doctors in Korea over the doctors in New Zealand. A woman had an operation for tympanitis (inflammation of the middle ear) at a public hospital in New Zealand after waiting seven months, but it did not improve. She was looking forward to another operation in Korea and stated without hesitation, "I would choose medical skills in Korea if I had serious illness" (IN15:297). Another woman spoke about her healthcare experiences with GPs, “They [GPs] don't seem to know much. They couldn't figure [symptom] out quickly" (T02a:68). One female in her 50s opined that: 
The doctors in Korea have survived serious competition to become doctors from a population of 50 million and developed their skills through countless practice there. New Zealand has a relatively small population and market compared to Korea, thus the doctors in New Zealand would be less skilled and experienced compared to the doctors in Korea. That is why the doctors in Korea may be better than the doctors in New Zealand, I think. In this regard, I trust the doctors in Korea more [than the doctors in New Zealand]. That's it. Even if the doctors in New Zealand are smarter than the doctors in Korea, they cannot be better than the doctors in Korea with regard to experience and skills. (IN01:299)

It was the common analogy amongst the participants: the better medical skills in Korea were a result of more chances to practice, and the more chances to practice were simply due to the larger population in Korea. One participant spoke about his belief on healthcare.

No one would doubt that doctors' abilities and skills are the most crucial factors for healthcare. Anyone with life threatening health issues would want a great doctor, an excellent specialist no matter the cost. (IN03:113)

Participants who were anxious about the New Zealand doctors' experiences and skills were worried about the level of medical technologies in New Zealand as well. "The medical technology in New Zealand is far behind Korea” (G02a:240). "MRI scans came to New Zealand 10 years after Korea" (T07a:226). "The concern is the aged medical equipment rather than this country's healthcare system" (IN05:97). The medical equipment and technologies in New Zealand were compared to those in Korea and judged to be outdated. These concerns were accentuated when the participants had a positive healthcare experience in Korea. A man who had had an operation for his sinus infections in Korea a few years earlier gave his view on the Korean healthcare system.

The healthcare system in Korea is well specialised like a conveyor belt. The doctors' experience in the number of patients, which is the amount of practice or the degree of proficiency is also very important, I think. It is affordable in Korea to purchase expensive medical equipment because a big enough market has already been formed there. (IN05: 223) 
The health services in Korea, unlike in New Zealand, are largely privatised (approximately 94\%), so there is serious competition between health service providers to attract patients (Kwon et al., 2015). Commercially oriented health services are largely operated by large-scale hospitals in Korea with its population of 50 million. These hospitals purchase expensive medical equipment and employ advanced technology in order to meet consumers' (rather than patients) satisfaction and to achieve higher profits as well. Hospitals in Korea are reportedly some of the best hospitals internationally with the most advanced technology medical equipment amongst OECD countries (OECD, 2012). Korean immigrants were familiar with the commercialised health services with advanced medical technologies in Korea.

The healthcare system in Korea is well organised and very convenient. If I take a gastro photography in a hospital, they show me it graphically, in three-dimensional graphics. It will be given to me on a $C D$ in to keep personally. Besides, it will be done in a few days. It is really convenient. (IN14:301)

Participants whose healthcare positions were rejecting gave a similar opinion on medical skills and technologies in both countries whether their opinions were based on direct or indirect experiences. Korean doctors were more experienced and better skilled, and Korean medical equipment and technologies were more advanced than those in New Zealand. Many of them judged medical skills and technologies as top priorities in healthcare, and considered them as ideal standards in healthcare. Their decisions in health service choices and the assessment of the healthcare they received were significantly determined by these standards.

\section{Fast and effective healthcare with easy access}

Patients in Korea walk in to hospitals without booking and referrals, and get various medications and injections for even trivial illnesses. Their greatest concern is quick relief, regardless of the type of disease. Participants, who came from the same health service environment, considered Korean health services without access difficulties as fast and effective care; and chose the health services in Korea as an ideal healthcare option for them. 
People in Korea can go directly to a specialist, and get treatment that is fast and effective. Here [New Zealand], I have to book even to see a GP. Booking is not necessary in Korea. I can get an injection even if go to a hospital without booking. (IN03:73)

The participant, quoted above, added, "There is no quick relief even though I am sick. That is the most discomfort in here [New Zealand]" (IN03:96). Another woman said, "Here, if it is a cold they [doctors in New Zealand] don't respond like doctors in Korea. Just 'endure' or 'take time', these are their common responses", (IN20:445). Sometimes, participants who had expected the same services that they were used to in Korea had to bear their symptoms without treatment because the doctors in New Zealand did not consider their symptoms as illnesses.

My GP said that drugs reduce the immune system of the human body, which has self-healing ability.... I had to live with skin trouble for a month because I couldn't get any relief [from the GP's treatment]. I think, New Zealand puts too much faith in natural healing. (IN16:744)

A woman said, "I could get a prescription for medication, so I could get relief quickly in Korea. But here, just Panadol. They [doctors in New Zealand] hardly prescribe medication" (T03b:154). In the participants' point of view, the doctors in New Zealand tended to over rely on natural healing and their treatments were ineffective. Individuals' illness perceptions are a product of interaction within a social context; people respond toward illness according to how they practiced and experienced illness within their society (Mishra et al., 2013; Salant \& Lauderdale, 2003). The different illness perceptions between GPs and Korean immigrants, which were shaped by two different societies and two different healthcare systems, created a gap in their patient-doctor relationship.

A number of studies claim the understanding gap between patient-doctor as one of the major contributors to patients' dissatisfaction (Alrashdi, 2012; Harmsen et al., 2008; Hudson et al., 2012; Suurmond et al., 2011). In this regard, minority ethnic groups are more likely to be dissatisfied with the host health services because the host society may not have enough experiences or knowledge to understand them, and the ethnic minority group may not know the host society either (Harmsen et al., 
2008). Likewise, immigrants' unsatisfactory healthcare experiences have been found to be largely caused by communication problems such as miscommunication and misunderstanding (Harmsen et al., 2008; Hudson et al., 2012; Suurmond et al., 2011). Ethnic minority immigrants are less likely to be satisfied with the health service in their host countries, and it is usually due to insufficient understanding with their physicians regarding their healthcare (Harmsen et al., 2008). Participants assessed the health services they received in New Zealand based on the service they experienced in Korea, and their healthcare decisions and health service utilisation were affected by these assessments.

Without a gatekeeping role in the Korean healthcare system, commercialised hospitals offer patients various healthcare services which can be referred as "Onestop" ${ }^{\text {39 }}$ access service, and patients enjoy the freedom to choose doctors and hospitals according to their personal preferences (Tse et al., 2013). In contrast, participants in New Zealand were confronted with GP referrals, which were difficult to get but inevitable for specialist care, and long waiting times for the public services, even after the GP referrals. One participant booked an ultrasound scan due to his fatty liver and waited for two weeks after his GP referred him.

Most hospitals in Korea offer medical test services such as an ultrasound scan, but here not many places provide these services.... From booking to seeing a doctor takes quite a long time here [compared to Korea]....I must not get sick here. (IN11:181)

The mother of one participant reportedly felt pre-stroke symptoms at midday, but she decided to wait until evening. Her daughter (participant) explained, "My mother knew that the access to the ED service is almost impossible during the day. She also wanted to avoid the process [the GP referral]" (IN20:181). Her mother gained access to the ED service that night and had two operations; however, she did not recover from the stroke due to the late treatment. "I want to see a doctor as soon as possible when I am sick, and it won't be a problem in Korea" (IN15:488). "The procedures for healthcare in New Zealand are not as fast as those in Korea. For

39 "One-stop" access to health services provides a comprehensive range of services including internal medicine, various surgeries, and medical check-ups in one place (Tse et al., 2013). 
example, it took me one and a half years to get a spine operation [in New Zealand]" (T07:254).

When the participants were disappointed with the access difficulties in public services such as ED service and secondary and tertiary levels of care, and the long waiting times after access to the service, they turned away from the New Zealand healthcare system and chose the rejecting position as their approach to healthcare. One married couple went to the ED service due to the wife cutting her finger with a knife. The wife said, "We waited for about three hours there until one a.m." (T04:417). Her husband asked the medical staff about the reason for the delayed treatment and were told, "There are patients with more urgent situations than yours" (T04:421). Another participant spoke about her friend's husband who went to the ED service with a stomach ache.

It could have been treated simply but became peritonitis after the seven hours wait, so he was hospitalised for three weeks....There are always patients with life threatening issues at the ED, and doctors have to save their lives first. It is their mission saving lives. I understand that. But if it were me, I would be very upset. (G01a:488)

Participants were very anxious about their health and healthcare within the New Zealand healthcare system and it was mainly due to the restricted access and long waiting times. Accordingly, the great fear amongst the participants was delayed diagnosis and treatments.

No one can wait for five or six months if it was cancer. If I were the one who had cancer, I cannot wait. People say that Koreans don't have patience. But it has nothing to do with patience. If something needs to be done in a hurry, we have to hurry. (G03b:570)

A man who had a sinus operation in Korea because he could not access specialist care in New Zealand complained, "I know that it [public health services] is free. But how long do I have to wait to get the free healthcare?" (IN05:254). Another participant pointed out that, "To get free healthcare, you have to wait for a long time" (T04a:121). A female participant who had been diagnosed with thyroid cancer by a doctor in Korea while she was on holiday shared: "It took me only 10 days to 
finish everything, diagnosing, biopsy and operation....It wouldn't be possible in this country [New Zealand]" (IN16:73). Participants, who were dissatisfied with the New Zealand health services, strongly adhered to the fast and effective healthcare with easy access options in Korea.

\section{Regular comprehensive medical check-up}

Koreans in the research, particularly those who took a rejecting position, expressed a great belief in a regular comprehensive medical check-up in Korea. They were satisfied with this medical check-up programme in Korea and believed that their health could be secured by this routine check-up. One participant in his 40s who had resided in New Zealand for 15 years took a comprehensive medical check-up in Korea every second year, "In order to stay healthy, a routine comprehensive medical check-up is crucial" (IN03:206). He was assured of continuous Korean medical checks in the future even though he always got a clean bill of health. He asserted that the rapid response to disease would be wiser and to discover disease at the early stage the regular check-up is helpful. Another man who was also in favour of a comprehensive medical check-up in Korea stated, "I think, just knowing about my health [condition] itself is very useful. Treating a disease after its onset would be too late" (IN05:205). His colorectal polyp was found during a medical check-up in Korea, and he felt secure with this check-up programme because disease was prevented by this check-up, he believed. One married couple stated, "It might be a waste of money if the results of the tests were all good, but it relieves my anxiety. I don't need to be anxious anymore after that" (T04a:648).

Participants perceived the emotional and psychological relief after the comprehensive check-up in Korea as one of its benefits. At the same time, they felt insecure and anxious over their health due to the absence of a full body check-up programme in the New Zealand healthcare system. "I used to take a [comprehensive] check-up every year while I worked for a company for 20 years in Korea. But they [New Zealand] don't have that kind of programme. It is very weird" (T04a: 436). "I want to take various medical tests regularly, every six months or once a year", (T06a:141). One woman who could not afford to travel to Korea for this medical 
check-up, reported with anxiety in her voice, "All my friends in Korea take a total body check-up on a regular basis" (IN01:39). For Koreans, the medical screenings in New Zealand such as blood tests and breast and cervical cancer screenings, which were provided on a regular basis according to individuals' age and gender, were not enough. They felt these screenings were superficial compared to the Korean comprehensive medical check-up, which consists of 22 items of body checks and medical examinations ${ }^{40}$ (Lee \& Lee, 2010). "I took a blood tests because there wasn't a comprehensive check-up [in New Zealand]....Frankly, I can't trust it [the result] because it was just based on a blood tests" (IN15:145). "The provided medical tests here are not enough for us....There are various affordable medical tests in Korea in terms of preventive healthcare. But in New Zealand, they are available only when I get sick" (T06a:127).

Sometimes their desire and knowledge regarding medical check-ups that stemmed from the Korean healthcare system, conflicted with the doctors in New Zealand. One man said, "We, Koreans, believe that certain medical tests must be held periodically when we are over 40. But they [doctors in New Zealand] said we don't need these tests if we are not sick" (T06a:184). One female participant in her 50 s stated with a frustrated voice, "Once, I requested an osteoporosis test to my GP because I had joint problems. But the GP's response was that I was over reacting. Even though he knew my age and medical history" (T02a:25). There were complaints amongst the participants with respect to the procedure of screenings in New Zealand as well. Participants who were used to having a one-stop access without long waiting times for medical check-ups in Korea found procedural inconveniences in medical screenings in New Zealand. One woman reported, "Unlike hospitals in Korea where various medical check-ups are available in one

\footnotetext{
${ }^{40}$ Koreans are used to taking whole body check-ups regularly according to their age groups. People aged 40 or over in Korea are entitled to take routine "comprehensive body checks" every two years, and the cost is covered by the NHI (National Health Insurance Service, 2014). There are also various private comprehensive medical check-up programmes at private hospitals and the minimum price range is approximately NZ\$700-800 ("Medical screening," 2014). However, it varies depending on the types and the number of screenings, and hospitals as well. Many hospitals in Korea provide this check-up programmes. People book for the service and it generally takes approximately 2-3hours.
} 
place, here, we have to request each test separately" (T03b:230). Another female participant pointed out that:

Many hospitals in Korea provide a comprehensive medical check-up. They examine the whole body in three hours [at one hospital]....But here, I have to book and wait for days and days even if it was just one gastroscopy. Moreover, the cost is too high. (IN14:88)

Unaffordable high costs for elective medical check-ups in New Zealand were perceived as another hindrance.

In Korea, a basic comprehensive check-up including a gastroscopy costs me about NZ\$400-500.... But here, the gastroscopy alone costs more than NZ\$1000. If more tests were added up, the cost would be tremendously high. That's why I take a comprehensive test whenever I visit Korea. (IN03:6)

Despite the traveling time and costs, participants found benefits of a comprehensive medical check-up in Korea: preventing diseases, subsequent emotional and psychological relief, and procedural convenience and cost effectiveness compared to the New Zealand screenings. Participants did not visit Korea only for the comprehensive medical check-up. They took a comprehensive medical check-up when they visited Korea for some other purpose, such as visiting family and friends, a holiday, or for business purposes. In their view, preventive healthcare in the New Zealand healthcare system was lacking.

After experiencing dissatisfactory healthcare in New Zealand, participants put strong faith in the Korean healthcare system. They preferred healthcare in Korea with experienced doctors and advanced technology and viewed Korean health services (with its high level of access and freedom of choice) as fast and effective care. They felt secure with the routine Korean comprehensive check-up programme. Consequently, they considered Korean health services as ideal. This faith assured and reinforced their healthcare position of rejecting within the New Zealand healthcare system. 


\subsection{Attuning: Emergency care at public services}

While most participants began with the rejecting healthcare position due to adverse healthcare experiences in New Zealand, attuning took place in healthcare acculturation as a result of satisfactory health service experiences. Figure 5 displays the healthcare position of attuning and its pathways including the turning point (a) in the Healthcare Acculturation Theory. There were two pathways leading to the attuning phase. The first pathway was the transition from rejecting to attuning position; the participants who were rejecting had their turning point (a) after experiencing satisfactory healthcare and changed their healthcare position from rejecting to attuning. Another pathway was attuning as an initial position with some participants choosing this position due to positive healthcare experiences in New Zealand.

\section{Turning point}

The turning point (a) ${ }^{41}$ represented the most powerful moment in the process of healthcare acculturation (Figure 5). Participants who reached such a point experienced transitions, not only in their healthcare positions, but in their life beliefs and values as well. Their healthcare positions were that of rejecting before experiencing a turning point, and when the turning point (a) took place, their position shifted from rejecting to attuning; this means, the turning point (a) was a precondition in order to transit from the rejecting position to the attuning position. Participants generally experienced this turning point when they utilised emergency healthcare (rather than elective care) at public sectors (e.g., ED service and secondary and tertiary level public services). Emergency care that participants experienced at public services was kind and caring care with rapid attention regardless of money (free care), which was person-centred care ${ }^{42}$ that they perceived as affective and altruistic care. Particularly, participants who considered fast and

\footnotetext{
${ }^{41}$ Turning point (a) was when the participant's healthcare position moved from rejecting to attuning as a result of being influenced by positive healthcare experiences. A turning point in the Healthcare Acculturation Theory was found at three different stages with (a) being the major turning point. The other two turning points (b) and (c) are explained in the Section 5.8 Negotiating: Attuning but negotiating and rejecting but negotiating (p. 155), 5.8.1 Attuning but negotiating (p. 155), and 5.8.2 Rejecting but negotiating (p. 158).

${ }^{42}$ See the Section 2.14 Person-centred care (p. 60).
} 


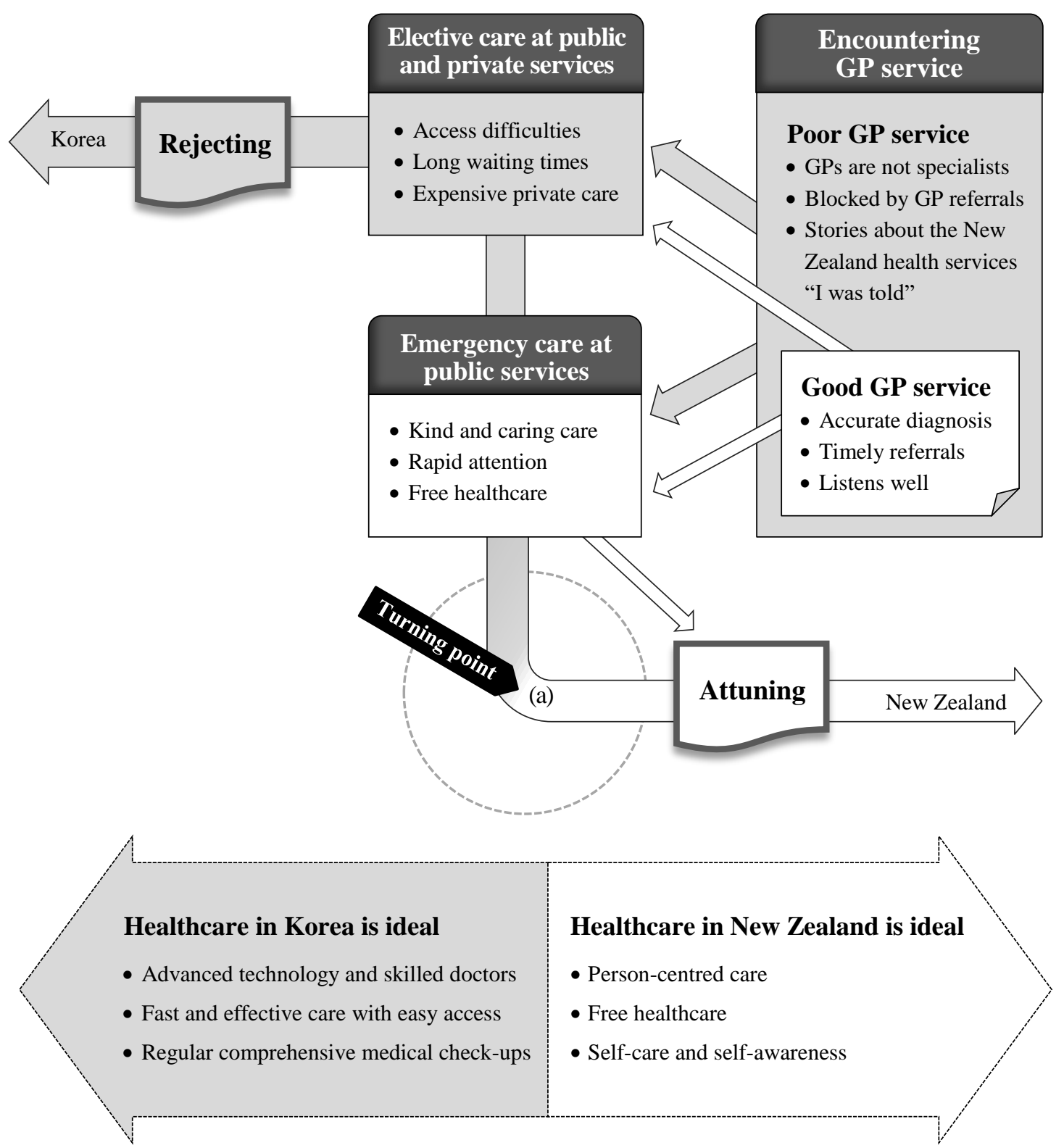

\section{Figure 5. Healthcare Acculturation Theory: Turning point and attuning}

effective healthcare with advanced medical technology in Korea as their ideal healthcare standards, were deeply impressed by the sincerity and kindness seen in the New Zealand public health services. Their healthcare perceptions and attitudes were changed after experiencing this type of care and their sense of values and beliefs were challenged by this healthcare experience. The turning point (a) occurred as a consequence of receiving care that Korean immigrants perceived as "altruistic" care. This turning point was not related to the participants' status (such as age, gender, and 
length of stay as immigrants) and their barriers to healthcare (such as cultural and linguistic incompetency and unfamiliarity of the host healthcare system). Rather, it was related to the ambience of health services (especially, the attitudes of the medical staff) that they had never experienced in Korea.

This turning point led participants with a rejecting position to turn back to the New Zealand healthcare system which they had previously abandoned. They were willing to adapt to the system and tried to embrace the problems that they faced within the New Zealand health services without complaints. Their negative perceptions and attitudes switched to positive; subsequently, their rejecting position was replaced by the attuning position.

\section{Attuning}

Once the attuning position took place in their healthcare acculturation process, attuning appeared in their daily lives in New Zealand as well. After experiencing the caring and genuine healthcare at ED service and secondary and tertiary level public hospitals, the participants, who believed in skilled doctors and advanced medical technology in Korea, changed their opinions. "I trust doctors in New Zealand" (IN02:311; IN09:351; IN13:72; IN21:735). "I have trust in the New Zealand healthcare system" (G01a:688). They moved to a position of trusting the New Zealand healthcare system over that of Korea. Even though they perceived medical skills in Korea as better than those in New Zealand, they chose the New Zealand health services over the Korean health services for their healthcare irrespective of minor or serious illnesses (life threatening). They tried to understand the approach of the New Zealand healthcare system and were willing to assimilate into it. Their "us and them" mentality was replaced by an "us" attitude, and they became more altruistic and expressed a desire to help society.

One participant whose parents-in-law had operations at a public hospital reported, "My father-in-law was extremely thankful to this country. He is making a 
donation to Barnardos ${ }^{43}$ and World Vision ${ }^{44}$ because he wanted to requite" (IN10:68). A young woman who had a breast cancer operation at a public hospital in 2014 said, "I am planning to join the Cancer Society Volunteer Driving Service because I was a beneficiary" (T01b:717). A similar opinion was found amongst the participants who experienced care that was heart-warming for them, "I am willing to pay tax" (IN06:61; IN09:105; IN20:128). They used to complain about high taxes in New Zealand before their change of heart. Participants' lives started to accommodate New Zealand society when attuning had taken place within the healthcare acculturation process.

\subsubsection{Healthcare in New Zealand is ideal}

Participants, who adopted the position of attuning, experienced transitions in many aspects of their lives. In particular, their beliefs on healthcare, which were their ideal healthcare standards, changed from the Korean to New Zealand standards. Participants, who preferred Korean health services because of advanced technology and skilled doctors, fast and effective healthcare, and regular comprehensive medical check-ups, considered the New Zealand health services as ideal for them. This was due to healthcare they experienced in New Zealand that was person-centred care with caring and kind medical staff, free healthcare that was not commercially driven, and self-care with increased self-awareness rather than being heavily reliant on hospital care (as in Korea).

\section{Person-centred care}

Patients' assessment of satisfaction with the healthcare provided is significantly determined by person-centredness in healthcare, such as caring, empathy, reliability, and responsiveness (Tucker \& Adams, 2001). Patients who experienced person-centred care ${ }^{45}$ demonstrate not only improved healthcare outcomes and increased satisfaction, but also changes in attitudes and behaviours

\footnotetext{
${ }^{43}$ Barnardos provides services to New Zealand children and their families who are at risk and disadvantaged (Barnardos, 2016, April 7).

${ }^{44}$ World Vision is an international evangelical Christian humanitarian aid, development, and advocacy organization (World Vision NZ, 2012).

${ }^{45}$ See the Section 2.14 Person-centred care (p. 60).
} 
(Hibbard \& Greene, 2013; Rogers, 1979). As a result of affective healthcare experiences at public services, participants expressed their favour, appreciation, and understanding toward the New Zealand healthcare system based on the healthcare they received. One participant compared his experiences of witnessing childbirth in New Zealand and in Korea.

In theatre, the patients in Korea feel that they are subjects of an experiment [to doctors] whilst doctors in New Zealand treat the patients with respect. It is a business, just work to do for them [doctors in Korea]. But they [doctors in New Zealand] care about the feelings of the patients....The atmosphere of the theatre is too cold in Korea but here [in New Zealand], it is like a party, it is more like home....[In theatre in Korea] many people come to check a patient but hardly greet the patient. Just the main doctor comes and explains briefly. But here, they [medical staff in New Zealand] explain kindly to the patient, and the atmosphere in theatre is not cold. I feel that the hospital bed is inside the house. The hospital bed is moved to a home. They [medical staff in New Zealand] care about the patient's feeling when the patient is lying naked on the bed in theatre. (IN22:05)

He was impressed by the warmth and kindness shown to his wife by health services at a New Zealand public hospital that he had not experienced in Korea. He preferred health services in New Zealand rather than in Korea even though he thought the medical skills in Korea were more advanced. His existing beliefs in healthcare were challenged and his ideal healthcare standards changed from Korean to New Zealand standards, which he saw as person-centred, rather than a cold conveyor belt of medical attention.

Patient satisfaction is a subjective matter as it is heavily dependent on their perceptions (Senić \& Marinković, 2013). Patients, in general, perceive healthcare according to the functional quality of the service (how the service is provided) rather than the technical quality (medical care) (Alrashdi, 2012; Senić \& Marinković, 2013), and it is because patients cannot easily assess the technical aspects of medical quality (Alrashdi, 2012). Unlike this, Koreans in this research who were affected by the functional quality of the New Zealand health services articulated that Korean medical skills are better than those in New Zealand without hesitation. They had believed in skilled doctors and advanced technologies in Korea, and these were their 
paramount concerns in healthcare. They still viewed the medical technologies in New Zealand as less advanced than in Korea and the doctors in New Zealand as less experienced and skilled than the doctors in Korea. However, participants who had experienced kind and trustworthy healthcare that was person-centred questioned their personal values and beliefs, and they came to believe that medicine was more than medical skills. One participant in his 50s opined:

I was told that the level of medicine in New Zealand is behind that of Korea, but I never experienced that. I went to a hospital many times due to my parents' condition rather than mine. Above all, they were very kind. It would be true that their medical facilities were outdated. And it would be possible that the medical equipment, which is used in Korea, is not in New Zealand. But I don't think that the latest medical equipment guarantees better health services. It may give some convenience....I have faith in doctors [in New Zealand]. (IN09:339)

Participants valued caring and kind healthcare over the medical skills and technologies in Korea. A woman in her 50s lived with her parents for 10 years in New Zealand, and both parents passed away six years ago (her father had lung cancer and her mother had blood cancer). She was grateful to the doctors for her parents' care, particularly her mother's doctors.

They [doctors] knew that my mother didn't have any hope at that time, I think. But they did their best for my mother even though they knew it. I was able to see it. I really appreciate them. Doctors in Korea would say, "Go home" under this circumstance. (IN20:199)

She was aware of her mother's impending death as were the doctors, but they acted as though her mother was going to recover. There was a mutual pretence between her as family of the patient and the physicians (Glaser \& Strauss, 1966). In Korea, traditionally under Confucianism ${ }^{46}$, children of dying parents tended not to inform the parents about their imminent death (Oh et al., 2004). They had an obligation to take care of their dying parents, even make decisions for them (with respect to treatment and the final end-of-life decisions), and it was considered as a

\footnotetext{
46 The traditional value and cultural norm in Korea were deeply influenced by Confucianism. Confucianism is not a religion rather it is a practical political and social doctrine that includes instructions of interpersonal behaviour (Śleziak, 2013).
} 
part of children's responsibility (Kwon, 2013). In Korean culture, children should provide every possible treatment and this was judged as demonstrating "caring" (Chung, Xu, \& Kwak, 2005). When her mother's condition became hopeless, the doctors' concern shifted from the patient to the family of the patient. Her mother's doctors pretended to treat her dying mother in order to comfort her (participant). Their compassionate care left her with strong faith in the New Zealand doctors and the healthcare system.

Koreans often complain about outdated medical equipment in New Zealand. Some complained about rusted equipment and so on. They are very anxious about those things, so they want to go back to Korea for their healthcare. In fact, I can understand them. Particularly dental care, it would be better in Korea because it is expensive here. However, I don't think that the medical equipment is that old enough to affect surgeries even though they are outdated. They [doctors in New Zealand] care about people's lives. I believe that they would change them if the outdated equipment became an issue for surgeries. (IN20:135)

Several participants experienced significant medical errors ${ }^{47}$ or traumatic healthcare incidents in New Zealand. They were losing a wife (IN06) and friend (G02a), stillbirth (IN13), miscarriage (IN07), and being left with lifetime health issues (IN08). A young mother who had had a stillborn in a New Zealand public hospital spoke about how she lost the baby. "Emotionally, it was terribly hard...because the ...the mistake wasn't mine. I noticed that [abnormal symptom] and told a midwife [several times] about that, but she continued to say 'no, it's not [a problem], just stay home," (IN13:127). Losing a baby is a devastating experience, especially if the tragedy was avoidable. "I know exactly when I lost the baby" (IN13:131), her eyes were filled with tears.

She also shared her experiences at the public hospital. "Around me, my mother-in-law and other Koreans strongly stopped me from seeing my baby" (IN13:87). She could not see her baby for three days even though she wanted to. Her

\footnotetext{
${ }^{47}$ Medical error is "the failure of a planned action to be completed as intended (i.e. error of execution) or the use of a wrong plan to achieve an aim (i.e. error of planning)" (Institute of Medicine, 2004, p. 30).
} 
hospital-based counsellor could not understand Korean culture and encouraged her to see her baby. Seeing the body of the deceased child facilitates parents' healthy grieving, and having an opportunity to say goodbye to the baby is important in the grieving process (Erlandsson, Warland, Cacciatore, \& Rådestad, 2013; Harrington \& Sprowl, 2012; Rådestad, Westerberg, Ekholm, Davidsson-Bremborg, \& Erlandsson, 2011). The hospital staff had put clothes on the dead baby and showed her the baby whenever she wanted to. This helped her to cope with her sorrow.

The counsellor explained that it would be better if I saw her and hugged her a lot...I did. Actually, it was really good....I could hug her as much as possible during the time that I had. Others [Koreans] may think I am strange. It could be strange for them, but I had time with my baby. I didn't have money at that time, but I wanted to have a funeral for her. When they [hospital] heard that, they even provided a funeral ceremony for my baby....At least I did something for my baby before she left, and I could let her go. (IN13:92)

Mothers who see and hold their stillborn babies have been found to have better psychological outcomes of the experience and lower levels of depression and anxiety compared to the mothers who do not have this interaction with the infant (Rådestad et al., 2011). The woman (participant) expressed her appreciation to the counsellor who encouraged her to see and have contact with the baby. After this experience, she realised that medical treatment is not only about wounded physical bodies but also about psychological and emotional hurts of patients. She came to believe that taking care of patients' feelings and emotions is as important as the medical skills. The hospital provided genuine caring for the patient who was saddened by the tragedy, and it changed the patient's appreciation of values in health and health services. After the interview, she said that the hospital gave her the baby's birth certificate with her baby's handprints and footprints. She said, "She [the baby] is my family, I feel that".

She did not want to blame anyone as she was grateful for the health service that she experienced at the public hospital after the stillbirth. "During the time, while I was in anguish, this country provided me with a lot of support" (IN13:106). The care and support provided by the hospital (encouraging her to see the baby, treating 
the baby as if alive and providing a birth certificate to comfort the mother, counsellor and interpreter services, and funeral service) comforted the mother greatly even though she was in an emotional and psychological traumatic state after the stillbirth. She was deeply touched by the genuine and empathetic support and care she received at the public hospital. The power of kindness rendered her forgiveness. In the middle of the interview, she said, "What if it happened in Korea?" (IN13:108). She assumed that she would have spent most of her time trying to figure out whose fault it was and be busy blaming others.

Another participant who was pregnant at age 39 experienced a miscarriage. In her third month of pregnancy, she saw blood on her underwear and reported it to her GP. 'My GP said, 'it's alright. Such a thing may happen.' I had to trust him because he was the doctor not me [upset and distressed voice]" (IN07:176). She went to a public hospital when her bleeding became serious and she miscarried.

When I ran to the hospital with serious bleeding they immediately took care of me, even washed me. They did everything for me....They phoned me [after she was discharged from the hospital] continuously to make sure that I got home safely. They checked on me for a day or two. (IN07:229)

She was disappointed with the miscarriage and in her GP. However, she felt that the healthcare she experienced at the public hospital was genuine, and appreciated the responsiveness of the medical staff. She reported, "I visited a public hospital three times [with other emergency issues], and every time they responded immediately. They never wasted even a second" (IN07:202). These healthcare experiences at the public hospitals enabled her to consider the GP as a person who can make a mistake, even though she could not trust the GP anymore and her expectations towards the GP service were lowered after that.

Participants who had experienced medical errors in New Zealand compared them to the medical errors in Korea, and the comparison was not only about the errors themselves but also about the attitudes of the medical staff and the ambience of health services. Their responses to the medical errors that they confronted were affected by the sincerity of the medical staff who provide the service and the caring 
and empathetic care they received. Present literature on patients' responses to medical errors found that patients and their families' decisions to litigate were determined not only by physical harms or injuries, but also by the attitudes of medical practitioners (Ambady et al., 2002; Huntington, \& Kuhn, 2003; Mazor et al., 2006; Mazor, Simon, \& Gurwitz, 2004). Korean immigrants who came from the commercially driven healthcare system in Korea felt the healthcare experienced at the public hospitals in New Zealand as empathetic and altruistic health services in a person-centred way.

\section{Free healthcare}

In the highly competitive medical environment in Korea, the private healthcare providers offer patients various health services with a range of healthcare options. While patients in Korea are given almost unconstrained freedom of choice of providers, treatment methods, and the type of ward in hospitals, medical cost is patients' share within the fee-for-service (FFS) payment system in Korea (Chun et al., 2009; Jones, 2010). Healthcare providers in Korea are paid dependent on the quantity of medical service provided to a patient under the FFS system. They tend to encourage patient's healthcare consumption; particularly, the uninsured services that involved high cost advanced medical technologies and expensive medicines to maximise profit (Kwon et al., 2015).

Koreans in the research were familiar with their role as the consumer at the Korean medical market. They commonly stated, "Doctors in Korea are like businessmen" (T05a:566). "In Korea, if we don't have money we can't go to hospitals" (IN13:476). "It is just commercial rather than caring for people" (G01c:592). Many participants who had experienced the public hospital care in New Zealand remembered the moment when they were discharged from a hospital. " $A$ nurse told me, 'You can go home now'. What should I do? It was a very awkward situation. I should pay though. But the nurse said, it was all free” (IN22:195). "I asked my husband to pay, and he came back and said, we can go home [without paying]. I said, 'How can we leave just like this?' So we waited. A nurse came and asked, why we were still there [laugh]" (IN20:112). Koreans, coming from a profit- 
driven healthcare system, were impressed not only by the New Zealand free health services but also medical staff who provided sincere care even though the services were free. They expressed great appreciation toward medical staff in New Zealand.

At that time, it wasn't a good situation financially....But I could have a lot of comfort due to the free healthcare that my husband received including the low medication fee. The healthcare was free but they [doctors] were not superficial. It wasn't only free health service, it was genuine care as well. I came to think "this country is quite attractive". (IN18:118)

A man reported, "Although hospitals provide free care, these people [medical staff in New Zealand] don't make us uncomfortable, and they are not haughty" (G03c:382). One participant whose sister received proper treatment due to the fast ambulance service and the medical staff's rapid response at a public hospital reported, "It is very different to Korea. Without money, we wouldn't get healthcare so quickly like that or we would die in Korea" (G01a:89). As participants became more affected by free healthcare in New Zealand and inspired by the genuine attitude of medical staff, they criticised the doctors and commercial health services in Korea. "Money is a top priority in Korea and Korean hospitals are totally business. At least, doctors here [New Zealand] seem to care about people's lives” (IN01:307). "Patients are not patients in Korea. They are just money [for the doctors]" (T05a:569). A man in his 30s who had recently experienced health services in New Zealand and in Korea as well indicated that, "In Korea, there is an extra fee for patients to pay. The patients who didn't pay the extra, they will be somewhat excluded at the doctor's routine rounds. The doctors are less likely to talk to those patients" (T01a:128). A similar experience was reported by another participant. She stated in an upset voice,

I was surprised when I heard that doctors in Korea take bribes from patients....Even my mother gave her doctor about NZ\$100 last time....And one, I know, who had a breast cancer operation in Korea, told me that she gave about NZ\$300 to her doctor for special favour. (T05a:568) 
Unlike the doctors' attitudes in Korea, which the participants experienced, one female participant who had a breast cancer operation at a public hospital in New Zealand reported, “The nurse told me, 'Don't worry about money. From now on, you should only worry about your health', I almost felt like crying. If it was in Korea, I can't even imagine it" (IN22:102). Participants who had healthcare experiences at public hospitals in New Zealand also found that the level of healthcare services they received at public hospitals eased the burden of patients and their family. In Korea, hospitals do not offer routine daily care services for patients such as help with eating bathing, and dressing (Park et al., 2012). Thus, one of the ill patient's family members usually has to take a caregiver role or they hire a caregiver for the patient. In this situation, being a patient in Korea is quite a heavy burden for the patient and for the family as well. "If it is cancer, on some occasions, we have to sell the house in Korea" (IN17:167). A participant who had experienced healthcare in Korea recently stated,

When I was ill I was very sorry [for my family], because of me....It is too difficult to manage if one of the family members is sick in Korea. Here [New Zealand], it seems that the family does not much care about the family member who is ill because hospitals take care of them - even eating and bathing. But patients in Korea need somebody for their support. (T01a:490)

One woman had to leave her husband at a public hospital in New Zealand because a nurse asked her to leave; besides, she found that there was no place for her to stay at the hospital. "They said, don't worry. Go and take a rest at home.... When I came back to the hospital, my husband looked happy because they took care of him so well" (G01b:658). As immigrants evaluate the host health services that they experienced based on their previous healthcare experiences in their home country (Lien et al., 2008; Mead \& Roland, 2009; O'Donnell et al., 2008), Korean immigrant's healthcare assessment in New Zealand was also influenced by the healthcare received in Korea. Koreans perceived the healthcare they experienced in New Zealand subjectively, according to their personal perceptions and expectations derived from their healthcare experiences in Korea. This means, it would vary depending on individuals and nations. It is apparent that the healthcare participants 
experienced at a public hospital was more than free healthcare for them. It was caring and inspirational care that they perceived as altruistic, and this level of healthcare had an influence on their emotional and physical healing. If they trusted the health service in Korea because of the medical skills and technologies; now, they put their faith in the New Zealand healthcare system because they believed that medical staff provided selfless care. One participant said, "I trust them. I know, they will take care of me" (IN02:329).

\section{Self-care and self-awareness}

When the Korean participants had experienced the public health services and were affected by empathetic and altruistic healthcare practitioners, they came to trust and tried to fit into the New Zealand healthcare system. Subsequently, changes in health-seeking behaviours took place amongst them. Participants reported that they did not go to GPs for minor illnesses such as colds or headaches because they knew that they would not get anything from GPs for these symptoms except a prescription for Panadol. In the past, they were also disappointed with the GP service and anxious about their health and healthcare as the participants who were in a rejecting position felt. While they lived in Korea, they were used to getting prescriptions with strong medications (including oral and injection antibiotics) from hospitals, and they were even able to purchase various medications from local pharmacies without prescriptions $^{48}$.

“Quick relief" from illnesses was their main concern even though they were trivial symptoms and it was believed by them to be the right way to deal with illnesses. This belief, the participants brought from Korea, was conflicted when they faced the New Zealand healthcare providers. One married couple visited a GP due to a wife's deteriorated cold symptoms. When the husband heard the GP say "Take Panadol and drink more water!", he felt frustrated. He replied, "It won't solve the problem. We didn't come for that [Panadol and drinking water]. Give us that, give us antibiotics!” (T04a:88). Previous studies (Choi, Park, Lee, \& Kwon, 2012; Norris et al., 2010) indicated the overuse and misuse of antibiotics (oral and injectable) in

\footnotetext{
${ }^{48}$ See the Section 1.4.3 Healthcare system in Korea (p. 11).
} 
Korea as a serious matter, and Koreans' low level of knowledge and misguided belief on antibiotics contributed to this behaviour. When the participants came to New Zealand they came with the health-seeking behaviour they practiced in Korea. They expected fast and effective healthcare with easy access to specialists and with prescriptions for strong medications (including oral and injection antibiotics) in order to have quick relief. Their healthcare needs and expectations, which were derived from the Korean healthcare system, were largely unmet within the New Zealand healthcare system.

However, once their negative perceptions of the New Zealand health services changed after experiencing the turning point, their healthcare positions shifted from rejecting to attuning, and their attitudes and behaviours were also influenced by these changes. A woman who had been resident in New Zealand for 21 years reported, "What I learned since I lived here, like a cold, it will disappear if you take a break and sleep well. Because it is usually caused by fatigue...If we have a rest, it will disappear" (IN10:442). The participants also spoke about their health as they perceived it: "Nowadays, I hardly catch a cold due to my improved immune system" (IN07:17); "My kid and I noticed that we hardly get sick now...I think, my kid became healthier after moving here" (IN12:242). They acknowledged their improved health during their residence in New Zealand and believed that it was a result of self-care without taking injections and strong medications. There were no special self-care skills or strategies found amongst them; rather than exercise, diet and daily rest, or drinking hot lemon tea and lots of water, and taking a rest and a Panadol for a cold.

According to the WHO (1983), self-care is "a means whereby people take much greater responsibility for their own health based on an understanding, ...of what health is all about, how to promote it, what damages it, how to protect it, and what to do when it does go wrong” (p. 1). Participants who were interested in selfcare also displayed their increased self-awareness of their own health: "I am much more aware of my health now than in Korea. I am concerned and care about diet, exercise and hygiene" (IN19:223); "I have to take care of myself. My attitude towards health has changed since Korea" (G03b:530). This participant also pointed 
out the reason why she was heavily reliant on hospital care in Korea: "The access was available at any time and getting a prescription for medication was too easy in Korea. These easily made us depend on doctors for our health" (G03b:492). Of note, the following was the views on doctors reported by the participants whose healthcare perceptions have changed: "We, Koreans, think a doctor who gives quick relief is a good doctor" (G02a:575); "When my child had a cold in Korea, my mother-in-law easily became impatient if she couldn't see any changes after a week of visiting a doctor. She used to urge me to find another doctor because she considered the doctor not good enough" (IN10:449); "Doctors in Korea readily provide a painkiller if someone is sick...they [doctors in New Zealand] wait and look first if it is not life threatening. This is the right way to go, I think" (IN04:160). Participants viewed themselves (Koreans) as sensitive to their own health but heavily dependent on hospital care, and doctors in Korea simply responded to their patients' demands.

Knowing oneself as well as one's health is primary to self-care, and the outcome of self-care is not only an increased sense of self-awareness, but can also result in changed behaviour (Richards, Campenni, \& Muse-Burke, 2010). Once their awareness of health increased, they began to develop a sense of responsibility for their own health. "There are diseases that can be prevented if we try to avoid them.... We should take care of our health ourselves. We shouldn't be reliant on hospital care too much" (IN07:246). The responsibility for their own health had shifted from their doctors to themselves. They might have lost their agency in terms of choices in health services within the New Zealand healthcare system; however, their autonomy (their own health control) increased when they took responsibility for their own health. The different healthcare orientations in Korea and New Zealand which influenced the health-seeking behaviour of Korean immigrants were compared by a participant.

Hospitals in Korea used to prescribe a lot of medications and injections in order to give quick relief. So if they don't take these medications, they feel uneasy because they feel, the disease would get worse. This anxiety urges people to take more medicines and injections. However, doctors in New Zealand pursue natural healing ${ }^{49}$. If they want to be

\footnotetext{
${ }^{49}$ Participants often used the term "natural healing" to mean self-care. They confused managing minor illnesses (e.g., a cold and headaches) through self-care instead of hospital medical care (such as
} 
popular with people they would prescribe strong medications to give quick relief, but they don't do that.... Their prescriptions never destroy or weaken our immune system. (IN07:319)

Participants who were in the attuning position began to think about healthcare that was good for people. A mother of two children reported, "they [doctors in Korea] overuse medications. But they [doctors in New Zealand] don't give medications. I really disliked it at first....Now I know, it was not a good thing to take a lot of medications even though it was easy to have" (IN14:207). They perceived self-care practice as a good health-seeking behaviour and believed that their health would benefit from it. A female participant spoke about her view on the New Zealand healthcare system:

The healthcare system in New Zealand is not very highly advanced like Korea. But they look for a way, which will benefit us.... Even if it takes a little longer, they look for a way that is good for one. In Korea, we go directly for antibiotics and injections for a cold in order to get quick relief. Here, it is not like that. They prefer to wait and try to heal naturally. (G01a:742)

Participants with an attuning position changed their health-seeking behaviour from being heavily reliant on hospital care to self-care with increased self-awareness and taking responsibility for their own health. Unlike them, participants who were in a rejecting position adopted self-care because they felt that they did not have a choice other than that which was offered in New Zealand. Further, they practiced self-care to protect themselves against diseases, so that they could avoid utilising the New Zealand health services.

Participants whose positions were attuning chose the New Zealand health services over the Korean health services for their healthcare practice. For them the healthcare in New Zealand is ideal (person-centred care, free care that is not commercially driven, and self-care with increased self-awareness rather than being heavily reliant on hospital care). They valued the New Zealand health services over

injections and strong medications) as natural healing even though they took some medications from pharmacies (e.g., Panadol). 
the fast and effective Korean healthcare not just because of the effect of self-care, but the trust they put in the New Zealand healthcare system and people in the system who care about patients sincerely. The more they attuned to the New Zealand healthcare system, the more harmony and balance they experienced in their daily lives in New Zealand.

\subsection{Negotiating: Attuning but negotiating and rejecting but negotiating}

Negotiating was an approach taken by participants who were in the rejecting or attuning positions. They negotiated when their healthcare options were limited, and they made healthcare decisions in order to have the best outcomes based on the situation that they were confronted with, such as waiting times, the type of diseases, healthcare cost, and time and cost for travelling to Korea. Their decisions were guided by rational thought and intention rather than their healthcare positions (which implied their healthcare perceptions and preferences), and their health-seeking behaviours were influenced by these decisions. There were two different types of negotiating positions: "attuning but negotiating" and "rejecting but negotiating". While the attuning but negotiating position transitioned from the attuning position, the rejecting but negotiating position moved from the rejecting position. Figure 6 illustrates these two different negotiating transition pathways in the healthcare acculturation process including the two turning points (b) and (c). As shown, the two turning points (b) and (c) occurred after negotiating took place. This means that these two turning points were not a precondition to the transition in healthcare positions like the turning point (a). Rather, they were merely the consequences of the negotiation and therefore did not have a strong impact on their healthcare acculturation process.

\subsubsection{Attuning but negotiating}

Participants who took an attuning but negotiating position had experienced transitions: rejecting $\rightarrow$ attuning $\rightarrow$ attuning but negotiating, or attuning $\rightarrow$ attuning but negotiating. Their healthcare perceptions and preferences were similar to those of the participants in the attuning position. They appreciated the caring and trustworthy 

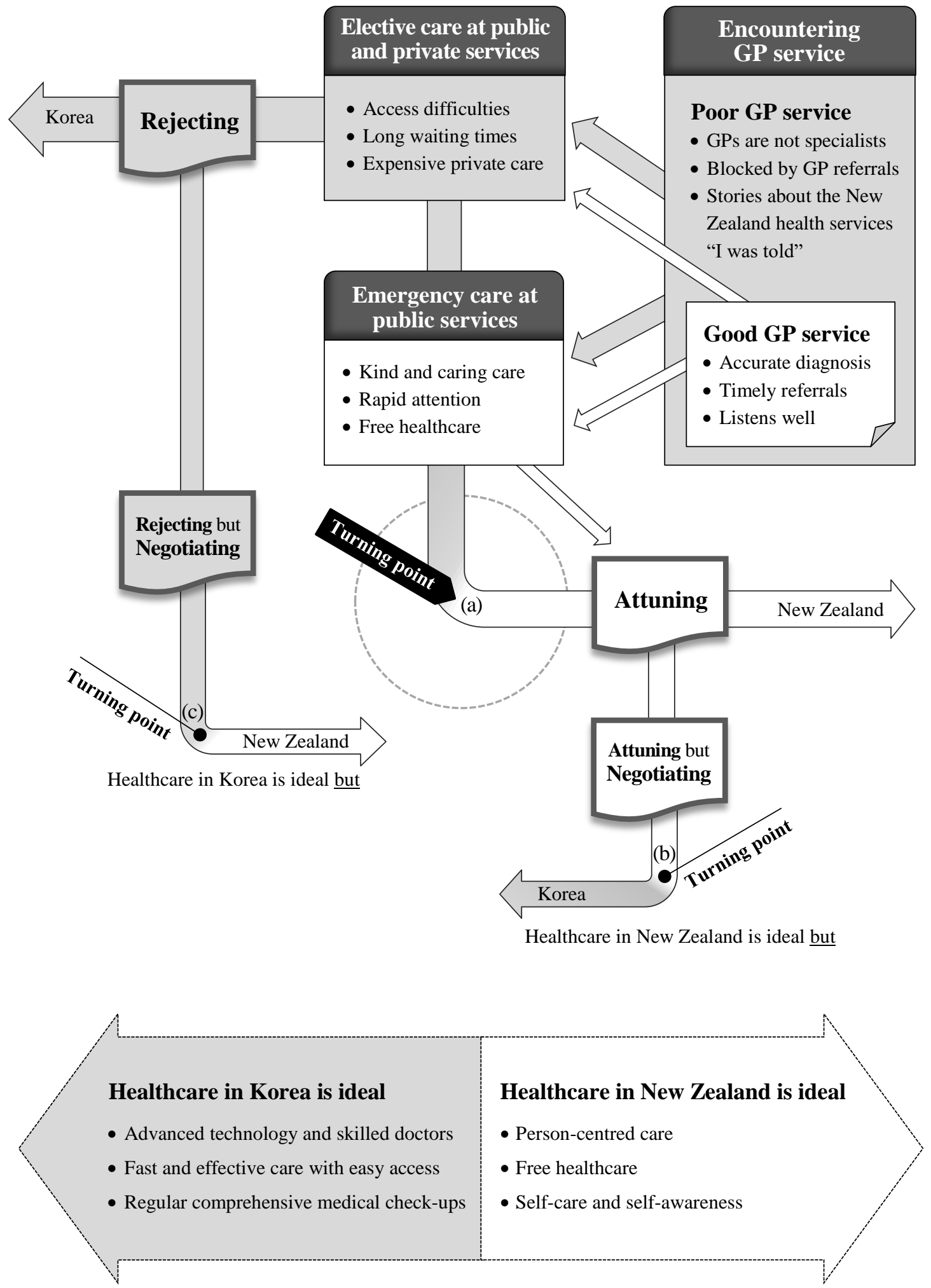

Figure 6. Healthcare Acculturation Theory: Attuning but negotiating and rejecting but negotiating 
healthcare practitioners and preferred to have health services in New Zealand. The healthcare in New Zealand was ideal for them. However, they would consider the healthcare options in Korea if they had to wait too long for the New Zealand services and/or the disease was extremely rare (considering the medical skills in both countries according to the type of disease). A turning point (b) occurred when the participants negotiated their healthcare options, and their healthcare position transitioned from attuning to attuning but negotiating. Although their healthcare position shifted, their perceptions and preferences remained. Therefore, in the process of healthcare acculturation, the turning point (b) from attuning to attuning but negotiating was not as influential as the major turning point (a) from rejecting to attuning.

For example, one participant experienced the turning point (a) in her healthcare position during her healthcare utilisation for her breast cancer at a public hospital in New Zealand. She was impressed by the kind and authentic care there, and consequently her healthcare position transitioned from rejecting to attuning. However, when her husband was diagnosed with Eosinophilic Granulomatosis with Polyangiitis (EGPA) ${ }^{50}$, and she learned that this disease was extremely rare (its incidence in New Zealand was approximately three cases/million per year) (Beasley, Bibby, \& Weatherall, 2008), they chose the healthcare option in Korea.

The breast cancer surgery in New Zealand is widely well reputed. But the disease [EGPA], in our opinion, would have a lack of clinical trials in New Zealand due to its small population. It would be better to go to Korea for its treatment if the disease is not well known. (IN21:539)

She believed that the decision was appropriate as Korean doctors would have more experience with the disease. Another woman in her late 40s, who showed a favourable attitude toward the New Zealand health services after experiencing public

\footnotetext{
${ }^{50}$ Eosinophilic Granulomatosis with Polyangiitis (EGPA), which is also known as the ChurgStrauss Syndrome, is a systemic vasculitis (massive eosinophilia in tissues and blood) associated with people who had or have asthma (Hasegawa et al., 2015). The diagnosis EGPA is often challenged by its multi-systemic characteristics with potential pulmonary, cutaneous, and/or neurological involvement (Guillevin, 2012). There are broad symptoms like asthma, weight loss, mononeuritis multiplex, sinusitis or polyposis, skin lesions, and lung infiltrates. It is life threatening and has no cure.
} 
hospital care, gave a metaphorical opinion regarding her healthcare options. "Let's suppose, the chocolate in Korea has better taste than that in New Zealand.

Definitely, I will choose the Korean chocolate if it is possible because I know the taste [laugh] " (IN18:333). Other participants, whose position was the attuning but negotiating, also spoke about their views on the healthcare options in Korea: "We, Koreans have choices. If I want to have healthcare in New Zealand I can take it here, and if I want it in Korea I can go there" (IN21:699); "I think it would be impossible to assimilate completely [into the New Zealand healthcare system] because we have an option. We can always go back to Korea if it is the solution" (IN20:501). Being a Korean in New Zealand was seen as a privilege with respect to healthcare for the participants who took the attuning but negotiating position due to having access to two different healthcare systems.

\subsubsection{Rejecting but negotiating}

Another position of the negotiating, rejecting but negotiating took place when the participants who were in the rejecting position confronted hindrances to utilising health services in Korea, such as time and cost for travelling, and fees for healthcare in Korea (rejecting $\rightarrow$ rejecting but negotiating). The participants, who took the rejecting but negotiating position held the same beliefs and attitudes as the participants in the rejecting position, so they still preferred to have healthcare in Korea and considered it as the ideal healthcare. When the healthcare in Korea was not available for them, they negotiated with the healthcare options in New Zealand. This means there was a turning point (c) in healthcare acculturation when their healthcare position changed from rejecting to rejecting but negotiating, but it was as minor as the turning point (b) in the attuning but negotiating position.

There were a number of reasons that led them to consider healthcare options in New Zealand: their financial situations, health status, age, jobs, and business. These reasons were directly related to the cost and time of travelling to Korea and the cost of healthcare in Korea. They calculated plus (pull) and minus (push) factors between both countries (Lee, 1966) and chose the healthcare option in New Zealand, taking the position rejecting but negotiating because it gave them better outcomes. 
Their healthcare decisions were based on their rational thoughts; "When I take into account the fact that I am not young anymore, I should try to fit into the New Zealand system, I think. When we were young and with money, it might have been a different story” (IN01:174); “Above all, I don't want to be a burden to friends and family in Korea....If I were sick, I will get better benefits in New Zealand than in Korea because I worked and paid tax for a long time" (G02a:261); "I am not a resident of Korea. Who is going to take care of me? Besides I don't have insurance in Korea” (T04a:1146).

In the positions in negotiating, the participants looked for the best possible healthcare option between the healthcare systems of New Zealand and Korea. Their healthcare decisions were based on their personal situations, not on their perceptions or present healthcare positions. This healthcare usage pattern demonstrated the transnational health service utilisation structure where immigrants seek care in both their country of origin and the host country (Wang \& Kwak, 2014).

\subsection{Summary}

Chapter five focused on a theoretical rendering of the findings of the research. The emergent Healthcare Acculturation Theory outlined Koreans' healthcare acculturating processes within the New Zealand healthcare system and provided a theoretical explanation of Koreans' health-seeking behaviours in New Zealand. In the Healthcare Acculturation Theory, Koreans' health-seeking behaviours were largely categorised into four different healthcare positions: attuning, rejecting, attuning but negotiating, and rejecting but negotiating. Each healthcare position portrays the individual's approach to healthcare including healthcare perceptions, attitudes, and behaviours including their levels of service utilization.

Korean immigrants who chose a rejecting position considered the healthcare services in Korea as an ideal healthcare option for them. They turned back from the New Zealand health services owing to dissatisfactory healthcare experiences with the GP service; access difficulties and long waiting times for public care; and the high cost of private care. They trusted skilled doctors with advanced medical technology and preferred fast and effective healthcare with easy access to specialist care in 
Korea. They favoured the Korean regular comprehensive medical check programme. Accordingly, they tended to avoid healthcare in New Zealand, and waited for the chances for healthcare in Korea.

The attuning position was chosen by Korean immigrants who had positive perceptions towards the New Zealand healthcare system. Their healthcare perceptions were influenced by healthcare experiences (emergency healthcare rather than elective care) at the public hospitals. The care they received was person-centred care with caring and kind medical staff, and free healthcare that was not commercially-oriented; and they perceived it as "altruistic". Particularly, participants whose position was rejecting experienced a turning point when they received healthcare at the public sector, and their present healthcare perceptions were challenged by the healthcare experienced. Once the turning point occurred in their healthcare acculturation their perceptions and attitudes changed and their healthcare position transitioned from rejecting to attuning. Participants in the attuning position valued the New Zealand health services over the Korean health services. They preferred healthcare in New Zealand and believed the New Zealand health services to be ideal.

The two different negotiating positions (attuning but negotiating or rejecting but negotiating) were found in the health acculturation process of Korean immigrants in New Zealand. They negotiated their healthcare options based on their personal situations and conditions in order to have the best outcome, even if their healthcare preferences were different. Koreans, whose healthcare position was attuning, considered healthcare options in Korea when their healthcare options in New Zealand were restricted by access, waiting times, the type of diseases, and healthcare cost. Their healthcare position moved from attuning to attuning but negotiating even though their preferred healthcare was in New Zealand. The rejecting but negotiating position was chosen by Korean immigrants who were in the rejecting position. They chose the healthcare option in New Zealand due to the cost and time of travelling to Korea and the cost of healthcare in Korea, even though they favoured health services in Korea. 
Korean immigrants' healthcare perceptions were influenced, not only by health services received in New Zealand, but also healthcare they experienced in Korea. Their healthcare positions were determined based on these comparisons of healthcare in Korea and New Zealand, and the chosen position directed their healthcare decisions and health-seeking behaviours. As healthcare acculturation is a continuous adaptation process, participants' healthcare experiences were ongoing. Their health positions were modified, enhanced, or transitioned during the healthcare acculturating process according to the healthcare they experienced. Korean immigrants' healthcare adapting processes within the New Zealand healthcare system were clarified and theorised into the Healthcare Acculturation Theory, and Korean immigrants' health-seeking behaviours were explained by this substantive theory developed from this research. The final chapter concludes the thesis by addressing the contributions, implications, and limitations of this research, as well as providing suggestions for future research. 


\section{Chapter 6. Conclusion}

\subsection{Introduction}

Korean immigrants encountered an unfamiliar healthcare system when they immigrated to New Zealand. During the healthcare adapting process their healthseeking behaviours were challenged and reconstructed as a consequence of interaction with the New Zealand health services. This research began with the broad research interest of: "what are the health-seeking behaviours of Korean immigrants in New Zealand and what factors affect these behaviours?' I investigated Koreans' healthcare experiences in New Zealand and in Korea including their perceptions of these experiences, and developed a substantive theory that provides a theoretical understanding of Korean immigrants' health-seeking behaviours and identifies their healthcare acculturation pathways within the New Zealand healthcare system.

This final chapter addresses the contributions this thesis makes to knowledge and to methodology and methods. It is followed by several implications of the findings from this research and the limitations that I perceived during and after the research. Recommendations for future research based on these limitations are suggested. It ends with concluding thoughts.

\subsection{Contribution to knowledge}

This research aimed to understand Korean immigrants' health-seeking behaviours in New Zealand and the factors affecting these behaviours. To understand this, Korean immigrants' approaches to healthcare and their healthcare adapting pathways within the New Zealand healthcare system were investigated and theorised. Since there is relatively little research related to health and healthcare of Koreans in New Zealand, their main concerns in healthcare and how they resolved these concerns are not well understood in New Zealand and even to Koreans themselves. Therefore, the main contributions made by this thesis are: a substantive theory developed to shed light on Koreans' health-seeking behaviours in New Zealand; the Healthcare acculturation theory which expands on the present acculturation theories; 
and a new understanding of the most influential contributing factor to healthcare which is the type of care that matters to patients.

The Healthcare Acculturation Theory developed from this research provides new and original knowledge of Koreans' health-seeking behaviours in New Zealand; making a contribution to the existing body of knowledge on acculturation and immigrants health. The health-seeking behaviours of Korean immigrants were categorised into four different positions: "rejecting", "attuning", "attuning but negotiating", and "rejecting but negotiating". Koreans chose one of these positions as their approaches to healthcare and the chosen position reflected their healthcare perceptions, attitudes, and behaviours including their degree of health service involvement in the host and home countries. Although the Korean immigrants were found to be in one of the four positions at any one point in time, their healthcare positions were not end-outcomes in themselves but rather transitional positions. Koreans' healthcare experiences were ongoing as acculturation is an on-going process (Ozer, 2013). There is an underlying assumption that there is no "acculturated" individual (Schwartz et al., 2010). These findings have implications for future interventions for Korean immigrants in New Zealand and offer a useful source of information to other ethnic minority groups; and will finally serve to improve their health.

The Healthcare Acculturation Theory elucidates Korean immigrants' healthcare adapting pathways within the New Zealand healthcare system. This theory expands on the present acculturation theories by providing the process of acculturation (rather than a static snapshot) with its reasons and consequences. Korean immigrants' healthcare acculturation process is explained with the contributing factors that influence their healthcare positions and pathways by this theory. Further, this substantive theory may be applicable to identify other ethnic immigrant groups' healthcare adapting processes and their health-seeking behaviours, even though it was specifically aimed to explain Koreans' health-seeking behaviour in New Zealand. As Glaser (1978) emphasised, the modifiability of theory to fit and work in practice needs to be tested by other researchers with other ethnic 
populations and under different healthcare settings (or by myself through postdoctoral research) to further develop the theory.

The outcomes of this research demonstrate that the most significant contributing factor in one's healthcare decision is the type of care that matters to the patients, beyond the individuals' barriers to healthcare. Another contribution made by this research is this new understanding that challenges the present literature on immigrants' healthcare. Prior research on immigrants' health and health-seeking behaviour largely focused on the barriers to health service utilisation in the host societies (Derose, Bahney, Lurie, \& Escarce, 2009; Derose, Escarce, \& Lurie, 2007; Leclere, Jensen, \& Biddlecom, 1994; Shin, Song, Kim, \& Probst, 2005), and indicated that the barriers generally stemmed from the gap between the healthcare systems of the host and home countries, as well as the cultural gap. These barriers were unfamiliarity of the host healthcare system, cultural and linguistic incompetency, and socio-economic disadvantages (Choi, 2013; Leduc \& Proulx, 2004; Lee, Kearns, \& Friesen, 2010). However, the outcomes of this research show that the major influences of immigrants' healthcare decisions and health-seeking behaviours are the type of healthcare that is related to the ambience of health services and the healthcare provider's attitudes rather than the barriers to healthcare that immigrants face in the host country.

Korean immigrants' health-seeking behaviour changed after experiencing healthcare at the public hospitals in New Zealand (with emergency care rather than elective). The healthcare the Koreans' experienced at the public service was kind, caring and empathetic, which is patient-oriented (person-centred) care, as well as free healthcare that is not commercially driven. They experienced healthcare in the public service that they had never experienced before. Korean immigrants' previous healthcare experiences were mainly with private providers in a commercially oriented competitive medical market in Korea (Kwon, Lee, \& Kim, 2015). They were used to being "consumers" rather than "patients" in their previous healthcare environment. They had the choice to select and purchase health services as consumers and enjoyed consumer power in their healthcare; they believed health services in Korea were an ideal service for them and postponed healthcare until they 
could go back to Korea. They rejected and abandoned the health services in New Zealand because they had lost their freedom and agency in the New Zealand healthcare system. However, when they experienced healthcare at the New Zealand public service they responded emotionally. They perceived that the health service received was altruistic and healthcare practitioners who provided the service were devoted. At that time, they could get attention and healthcare as patients, rather than consumers who had purchasing power with consumer rights. This impression rendered a turning point in their healthcare acculturation process and in their immigrant lives as well. Accordingly, their health-seeking behaviour was affected by this healthcare experience and their healthcare position also transitioned from rejecting to attuning.

Immigrants tend to assess their healthcare experience in their host countries based on their healthcare experiences in their home countries (Lien, Nafstad, \& Rosvold, 2008; Mead \& Roland, 2009; O'Donnell, Higgins, Chauhan, \& Mullen, 2008). This means, other ethnic immigrant groups in New Zealand may have different perceptions of the New Zealand health services; they may not feel that health services at the public hospitals are affective and altruistic. In this regard, it would be difficult to define the ideal health service that patients seek since patients perceive their healthcare subjectively. Nevertheless, it is crucial to understand what matters to patients (Rickert, 2012) because these matters influence their healthcare decisions and further, their health-seeking behaviours. There has been increasing interest in providing care from a person's perspective with respect to the person's own values, experiences and knowledge; accordingly, person-centred care has been acknowledged and adopted as an ideal healthcare approach in many countries (Epstein \& Street, 2011; Leplege et al., 2007; McCormack, Karlsson, Dewing, \& Lerdal, 2010).

The findings of this research do not articulate the ideal healthcare system or health services for people, but explain what matters to the patients from the patients' perspectives. Korean immigrants experienced altruistic care at the New Zealand public sector whether it was truly altruistic or not, and their health-seeking 
behaviours and healthcare decisions were significantly impacted by these experiences.

\subsection{Contribution to methodology and methods}

Acculturation research has been predominantly quantitative while this research, focusing on Korean immigrants' healthcare acculturation process, has employed an inductive qualitative approach. I explored Korean immigrants' healthcare experiences and perceptions, and concerns and expectations in healthcare in their own settings. My intention, in using the qualitative approach, was to develop a substantive theory that provides an in-depth understanding of the issues of the participants. The rich and detailed data were collected by the open-ended face-toface interviews, and they were analysed and theorised in order to provide an explanation of theoretical understanding. As a result, the Healthcare Acculturation Theory was developed with a deeper insight into Koreans' healthcare acculturation process and health-seeking behaviours in New Zealand.

Another contribution of this thesis to methodology and methods lies in how I managed the myriad of challenges and difficulties in identifying a variation of grounded theory that enabled me to develop an authentic approach to using this methodology, finally following Glaser's classic grounded theory with emergent fit approach. Since this research demonstrates the use of grounded theory in a research involving two different languages, a further contribution to methods is related to how I coped with the complexity of carrying out data collection and analysis in two languages (particularly, during the coding and theory development processes); and how translation became a foundation for the conceptual theory development and was integrated into theory building with analytic power.

\subsection{Implications}

As most immigrants experience changes in their health-seeking behaviours as part of acculturation (Choi, 2013b), Korean immigrants' health-seeking behaviours were reconstructed and transitioned during the process of healthcare acculturation. 
According to the findings of the research, Korean immigrants were affected by healthcare at the public hospitals that was sincere, empathetic and responsive care delivered in a person-centred way, beyond the cultural gap, language difficulties, and the difference between healthcare systems. These outcomes from this research have several implications for healthcare practice and policy, as well as immigrants themselves and their communities.

First, Person-centredness in healthcare should be encouraged in practice as individuals' healthcare decisions are directly affected by the healthcare practitioner's attitudes and the ambience of health services. Second, in order to provide more person-centred care, healthcare practitioners need to understand not only the patients' symptoms but also their needs and seeking behaviours, as patients seek not only medical treatment but also quality empathetic care and trusting relationships with their medical practitioners. It can result in better outcomes of patients' health and a reduced burden on health services; in other words, it can benefit both patients and healthcare providers. Third, therefore, efforts should be concentrated in improving interpersonal skills in the healthcare setting to understand patients' concerns and to know what matters to them. Healthcare practitioners should look for ways to develop their interpersonal skills, not just intercultural skills; and education and training programmes must teach them how to improve these skills effectively.

In addition, although this research strongly emphasises the type of care that has an impact on patients, health literacy found amongst Korean immigrants should not be overlooked. Immigrants' insufficient information and understanding as to their entitlements to care and availability of health services in the host country influence their obtaining adequate and timely healthcare (Davies et al., 2009; Galanis et al., 2013). Therefore, increased attention is required to provide appropriate information regarding the New Zealand healthcare system; how the healthcare system works in New Zealand and a proper explanation of health services available to them. Further, education of Korean immigrants through a proper channel (like the Korean community ethnic network as a primary source) would be more effective, and will facilitate Korean immigrants' health service access. 


\subsection{Limitations and recommendations for future research}

Being a Korean researcher who had knowledge and an understanding of Korean immigrants was a benefit to this research (as I reported in the Section 3.4.2 The role of the researcher); however, it could also lead to potential researcher bias. I shared the same background and spoke the same language as the participants, had an insider understanding of the participants' experiences and their life stories, and empathised with them. I was at risk of being too close to the data when I found myself in their stories. To avoid this bias, I applied constant comparative analysis and theoretical sampling together with various memos and reflexively used a research journal throughout the research.

This research closely followed grounded theory methods. Sampling and data collection were guided by systematic application of constant comparative analysis with theoretical sampling methods as part of emerging theoretical ideas. As a result, the data were only from first generation Korean immigrants and not from younger Koreans who were born in New Zealand. Younger age groups may have different perceptions and experiences of healthcare in New Zealand. This could limit the research findings, even though generalisation was not a concern of this exploratory research. Additionally, participants in this research were recruited from Auckland and Wellington in the North Island. Koreans in the Canterbury Region, where $11.1 \%$ of the Korean population live (3,336 people) (Statistics New Zealand, 2014a), were not included. Therefore, it is recommended that future research regarding Korean immigrants' health-seeking behaviour in New Zealand includes second generation Korean immigrants or the younger age groups; and/or Korean immigrants in Christchurch (Canterbury Region). It would be of interest to compare the outcomes of this research with the findings from future studies focusing on different Korean immigrant generations and age groups, or different regions.

While the findings of this research were generated from the rich and detailed data collected from the Korean immigrants' healthcare experiences and stories, this research did not investigate the healthcare providers' perspectives on Korean immigrants as patients. Healthcare practitioners' perceptions of the health-seeking behaviours of Korean immigrants would extend the substantive theory developed in 
this research; especially, perspectives from GPs (General practitioners) who played a key role in Koreans' rejecting healthcare decisions, and doctors and nurses at the public hospitals who provided a turning point for Korean immigrants from rejecting to attuning. Therefore, future research should consider including other stakeholders who are involved in Koreans' healthcare and have influence on their health-seeking behaviours.

This research was to understand how Korean immigrants, who came from a very different healthcare system, reconstructed their health-seeking behaviours by adapting to the New Zealand healthcare system. It would be interesting if future research were to focus on New Zealanders' healthcare experiences in Korea, and explore their health-seeking behaviours and the changes within the Korean healthcare system; how New Zealanders in Korea perceive the Korean health services, what factors lead to changes in their health-seeking behaviours, and how their healthcare acculturation process in Korea is different to that of Korean immigrants in New Zealand. The findings of the possible future research, together with the findings of this present research, would provide a more comprehensive understanding of immigrants' health-seeking behaviours and the factors that contribute to their behaviours and healthcare decisions.

\subsection{Conclusion}

In this thesis I have made an important contribution to the existing body of knowledge on acculturation demonstrating new knowledge and a theoretical understanding of health-seeking behaviour, in the development of a substantive theory based on the experiences of Korean immigrants in New Zealand. How immigrants perceive their experiences of health services shapes their perceptions of the healthcare system of the host country; and these perceptions have major influences on their healthcare decisions and health-seeking behaviours, and their immigrant lives as well. According to the findings of this thesis, the most influential factor contributing to the health-seeking behaviour of Korean immigrants was the type of care that they perceived as affective and altruistic. This understanding challenges the present literature on immigrant's health and healthcare which stresses 
the unfamiliarity of the host healthcare system, cultural differences, and language difficulties as the main influences in immigrants' healthcare.

This research emphasises the attitudes of the medical staff and the ambience of health services that resulted in sincere, empathetic and responsive care as the major contributing factors to Korean immigrants' acceptance of healthcare. The "turning point (from rejecting to attuning)" represented the most powerful moment in the process of healthcare acculturation for these Korean immigrants. Participants who reached such a point experienced transitions, not only in their healthcare positions, but in their life beliefs and values as well, which went far beyond simply a shifting perspective but can be likened more to a "conversion". This was conversion to a new understanding of the experience of being a "patient", who is cared for in a person-centred way, where they came to understand New Zealand in a different way and were willing to assimilate, rather than remain consumers in a financially driven and "cold" Korean healthcare system. 


\title{
Appendices
}

\section{Appendix 1. Participant information sheet (Korean)}

\author{
VICTORIA UNIVERSITY OF WELLINGTON \\ Te Whare Wananga o te Upoko o te Ika a Mani

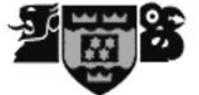 \\ 연구 참가자를 위한 정보
}

\section{연구 제목}

뉴질랜드 한인 이민자들의 건강과 관련된 행동·습관 연구

Examination of the health-seeking behaviours of Korean immigrants in New Zealand

\section{연구원}

김미경 박사 후보

Graduate School of Nursing, Midwifery and Health,

Victoria University of Wellington

저는 웰링턴 빅토리아 대학에서 박사과정 연구논문을 수행하고있는 박사과정 학생입니다. 본 연구는 뉴질랜드 한인들이 건강 관련 어떠한 행동과 습관들을 가지고 있는지 살펴보고, 이러한 행동 습관 형성에 영향을 미치는 요인들을 연구하고자 합니다.

연구 참가자: 한국인 만 18 세 이상으로 뉴질랜드에 거주하고 계시는 (18개월 이상) 영주권 혹은 시민권 소지자이면 연구에 참여하실 수 있습니다.

연구 목적: 본 연구는 뉴질랜드 한인 이민자들이 건강과 관련하여 어떤 행동과 습관을 가지고 있는지, 의료 서비스를 이용할 때 어떤 요인들이 의사결정에 지대한 영향을 미치는 지를 연구하고자 합니다

연구의 중요성: 뉴질랜드에 거주하고 계시는 한인들의 건강과 관련 - 어떻게 건강을 관리하고 유지하는지, 어떻게 의료 서비스를 이용하고 있는지에 대한 - 이해가 부족한 것이 사실입니다. 따라서 본 연구가 뉴질랜드에 거주하는 한인들의 건강과 삶을 이해하는데 도움이 되고, 더 나아가 이해를 바탕으로 하는 적절한 의료서비스 제공과 한인 이민자들의 건강을 증진을 도모하는데 역할을 하게 될 것입니다.

연구에 참여하시게 되면: 기본적으로 1 회 또는 2 회의 인터뷰 (개별 또는 그룹) 에 참여하시게 됩니다. 인터뷰는 보통 60 90분이 소요될 것으로 예상되나 이는 참가자들의 그때 상황에 따라 조정될 수 있습니다. 모든 인터뷰는 한국어로 진행됩니다. 모든 인터뷰는 녹음되며 녹음된 내용은 사본으로 옮겨져 영어로 번역 될 것입니다. 인터뷰 후, 요약 정리된 인터뷰 내용을 다시 한 번 구두로 확인 하실 수 있습니다. 언제라도 연구에 참여를 원치 않으실 경우에는 참여 취소가 가능하며 제공하신 정보는 인터뷰 후 2주 안에서는 철회가 가능 합니다. 
연구 참여 동의: 본 연구는 자발적인 참여를 기본으로 하며, 연구동의서에 서명을 시작으로 연구에 참여하시게 됩니다.

기밀 유지: 연구 참가자가 제공하는 모든 정보는 저를 포함하여 감독 교수님들과 오디오 녹음을 번역 하는 사람에게도 기밀로 유지 될 것입니다. 참가자의 신원은 익명으로 유지됩니다. 코드/가명이 참가자의 이름을 대신 할 것이며, 모든 메모와 인터뷰 녹음자료에도 코드/가명이 사용될 것입니다. 연구 결과가 게재된 출판물에 참가자의 이름이 언급되지 않으며, 신원이 밝혀질 여지가 있는 어떤 내용도 포함하지 않습니다.

연구 승인: 본 연구는 빅토리아 대학 인간윤리위원회 (Victoria University of Wellington Human Ethics Committee)로부터 승인을 받았습니다.

연구 결과: 논문은 웰링턴 빅토리아 대학 도서관에 보관 될 것입니다. 학술 저널에 연구 결과가 게시될 것이며, 뉴질랜드 한인 단체에 연구결과가 발표 될 것입니다.

데이터 보관 및 파괴: 모든 데이터는 패스워드로 접근이 제한되고, 역시 패스워드 처리가 된 컴퓨터 에서 10 년 동안 보관되고 그 후에는 파괴 될 것입니다.

본 연구에 관련하여 질문이 있거나 자세한 정보를 원하면 저 또는 연구 감독 교수님들께 문의하여 주시기 바랍니다.

\section{연락처:}

김미경 (Michelle Kim), 박사 후보

Graduate School of Nursing, Midwifery and Health, Victoria University of Wellington Phone: 044636662

e-mail: michelle.kim@vuw.ac.nz

Dr. Kay de Vries, 제1 연구 감독 교수

Graduate School of Nursing, Midwifery and Health, Victoria University of Wellington Phone: 044636650

e-mail: kay.devries@vuw.ac.nz

Dr. Kathy Nelson, 제2 연구 감독 교수

Graduate School of Nursing, Midwifery and Health, Victoria University of Wellington Phone: 044636138

e-mail: kathy.nelson@vuw.ac.nz 


\title{
Appendix 2. Participant information sheet (English)
}

\author{
VICTORIA UNIVERSITY OF WELLINGTON \\ Te Whare Wananga o te Upoko o te Ika a Maui

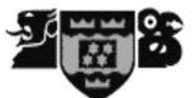 \\ PARTICIPANT INFORMATION SHEET
}

Title of the study

\section{Examination of the health-seeking behaviours of Korean immigrants in New Zealand}

Researcher

Mikyung Kim, PhD candidate

Graduate School of Nursing, Midwifery and Health, Victoria University of Wellington

Introduction: I am a Korean $\mathrm{PhD}$ student undertaking a research project that will lead to a doctoral thesis at the Graduate School of Nursing, Midwifery and Health, Victoria University of Wellington. The aim of the study is to explore the health-seeking behaviour of Korean immigrants in New Zealand.

Participants: You have been invited to participate because you are a Korean immigrant with either permanent residency or citizenship living in New Zealand and you are aged 18 or over.

The purpose of the study: The study aims to examine your health-seeking experiences and the factors affecting your decision-making related to healthcare when you are accessing health services.

Benefits and significance: The findings of this study will fill a gap in the knowledge and understanding of Korean immigrants' health-seeking behaviours in New Zealand. The study results will have implications for future interventions for Korean immigrants and will serve to improve their health and health care.

Participation: You will be asked to share your experiences by participating in one or more interviews which may be either individual or in a group. It is estimated that the initial interview will last 60 to 90 minutes. All interviews will be conducted in Korean. They will then be audio-recorded, transcribed and translated into English. You will be offered an opportunity to discuss a summary of your interview transcript. You can withdraw from the study or ask that certain information be removed up to two weeks after the interview.

Participant information sheet - Ethics approval 20449 Version 1: Examination of the health-seeking behaviours of Korean immigrants in New Zealand. Approved 18/12/2013 
Consent: Participation in this study is voluntary. To participate you will be asked to sign a consent form.

Confidentiality: Any information you provide will be kept confidential to myself, the supervisors and the person who transcribes the audio recordings of interview. Your identity will be kept anonymous and you will be assigned a code/pseudonym, and it will replace your name in all notes and transcriptions. The published results will not use your name, and no opinions will be attributed to you in any way that will identify you.

Statement of approval: This research project has received approval from Victoria University of Wellington Human Ethics Committee.

Study findings: The thesis will be deposited in the Victoria University of Wellington Library. It is intended that one or more articles will be submitted for publication in scholarly journals and that the results will be presented in Korean communities.

Data storage and destruction: The data will be password-protected and stored for 10 years in the password-protected computer, and then destroyed.

If you have any further questions or would like to receive further information about the project, please contact me and my supervisors.

\section{Contact details:}

Mikyung Kim (aka Michelle Kim), PhD candidate

Graduate School of Nursing, Midwifery and Health, Victoria University of Wellington Phone: 044636662

e-mail: michelle.kim@vuw.ac.nz

Dr. Kay de Vries, Primary supervisor

Graduate School of Nursing, Midwifery and Health, Victoria University of Wellington Phone: 044636650

e-mail: kay.devries@vuw.ac.nz

Dr. Kathy Nelson, Secondary supervisor

Graduate School of Nursing, Midwifery and Health, Victoria University of Wellington Phone: 044636138

e-mail: kathy.nelson@vuw.ac.nz

Participant information sheet - Ethics approval 20449 Version 1: Examination of the health-seeking behaviours of Korean immigrants in New Zealand. Approved 18/12/2013 


\section{Appendix 3. Participant consent form (Korean)}

VICTORIA UNIVERSITY OF WELLINGTON

Te Whare Wananga o te Upoko o te Ika a Maui

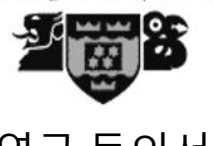

연구 동의서

연구 제목

\section{뉴질랜드 한인 이민자들의 건강에 관련된 행동·습관 연구}

Examination of the health-seeking behaviours of Korean immigrants in New Zealand

연구원

김미경 박사 후보

Graduate School of Nursing, Midwifery and Health,

Victoria University of Wellington

나는 본 연구에 대하여 설명을 들었으며, 연구에 참여하기 위해 할 일에 대해 잘 알고 있습니다. 나는 이 연구에 자발적으로 참여하고자 합니다.

나는 아래의 항목들을 잘 이해하고 있습니다:

- 나 자신을 보호하기 위하여 특정 정보를 변경 혹은 제외를 요청할 수 있습니다.

- 인터뷰 중 모든 질문에 대답할 의무가 없습니다.

- 언제라도 연구 참여를 취소할 수 있으며, 제공한 정보는 인터뷰 후 2 주 안에서는 철회할 수 있습니다.

- 모든 인터뷰는 녹음되며, 녹음된 내용은 사본으로 옮겨져 영어로 번역 될 것입니다.

- 내가 제공하는 모든 정보는 담당 연구자를 포함하여 감독 교수님들과 오디오 녹음을 번역 하는 사람에게도 기밀로 유지 될 것입니다.

- 연구 결과가 게재된 출판물에 내 이름이 언급되지 않으며, 내 신원이 밝혀질 여지가 있는 어떤 내용도 포함하지 않습니다.

- 연구 완료 시 요약된 연구 결과를 연구자에게 요청 할 수 있습니다.

- 모든 데이터는 패스워드로 접근이 제한 될 것이고, 역시 패스워드 처리가 된 컴퓨터에서 10 년 동안 보관되며, 그 후에는 파괴 될 것입니다.

이 연구에 참여하겠습니다.

서명:

참가자 이름:

날짜: 


\title{
Appendix 4. Participant consent form (English)
}

\author{
VICTORIA UNIVERSITY OF WELLINGTON \\ Te Whare Wananga o te Upoko o te Ika a Mani

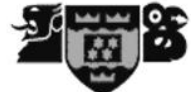 \\ PARTICIPANT CONSENT FORM
}

Title of the study

Examination of the health-seeking behaviours of Korean immigrants in New Zealand

Researcher

Mikyung Kim, PhD candidate

Graduate School of Nursing, Midwifery and Health,

Victoria University of Wellington

I have been given an explanation of this research project and I understand what will be involved if I participate in the study. My participation is voluntary.

I understand that:

- I have the right to request changes or to exclude certain information to protect myself.

- I do not have to answer all the questions during the interview.

- I may withdraw from the research myself or ask that certain information be removed (that I have provided) up to two weeks after the interview.

- All interviews will be audio-recorded, transcribed and translated into English.

- Any information I provide will be kept confidential to the researcher, the supervisors and the person who transcribes and translates the audio recordings of our interviews.

- The published results will not use my name, and that no opinions will be attributed to me that will identify me.

- I can request a summary of the research results when it is completed.

- The data will be password-protected and stored for 10 years in the passwordprotected computer, and then destroyed.

I agree to take part in this research.

Signature:

Name of participant:

Date:

Participant information sheet - Ethics approval 20449 Version 1: Examination of the health-seeking behaviours of Korean immigrants in New Zealand. Approved 18/12/2013 


\section{Appendix 5. Ethics approval}

TE WHARE WĀNANGA O TE ŪPOKO O TE IKA A MĀUI

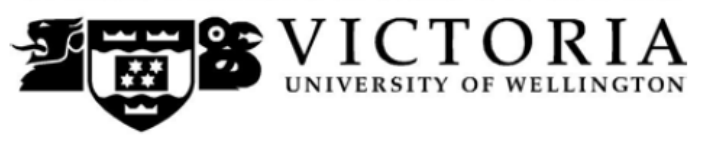

MEMORANDUM

Phone $\quad 0-4-4635676$

Fax $\quad 0-4-4635209$

Email_Allison.kirkman@vuw.ac.nz

\begin{tabular}{l|l}
\hline TO & Mikyung Kim \\
\hline COPY TO & Kay de Vries \\
\hline FROM & Dr Allison Kirkman, Convener, Human Ethics Committee \\
\hline
\end{tabular}

\begin{tabular}{l|l}
\hline DATE & 18 December 2013 \\
\hline PAGES & 1 \\
\hline
\end{tabular}

SUBJECT

Ethics Approval: 20449

Examination of the health-seeking behaviours of Korean immigrants in New Zealand

Thank you for your application for ethical approval, which has now been considered by the Standing Committee of the Human Ethics Committee.

Your application has been approved from the above date and this approval continues until 26 February 2016. If your data collection is not completed by this date you should apply to the Human Ethics Committee for an extension to this approval.

Best wishes with the research.

Allison Kirkman

Human Ethics Committee

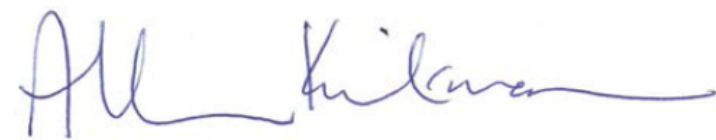




\section{Appendix 6. Interview guide}

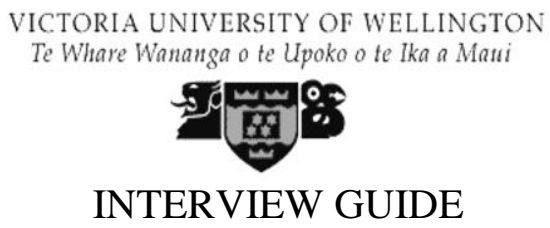

INTERVIEW GUIDE

\section{Title of the study}

Examination of the health-seeking behaviours of Korean immigrants in New Zealand

Researcher: Mikyung Kim, PhD candidate

Graduate School of Nursing, Midwifery and Health, Victoria University of Wellington

The following questions will be used to commence, guide, and give basic structure to individual and group interviews.

1) How do you perceive your health risk and needs?

2) What were your perceptions or behaviours, with respect to health risks and needs, before immigration, and since you have settled in New Zealand?

3) What have been your experiences in relation to your health needs in New Zealand?

4) What are the most influential factors in your decision-making to seek health care?

5) What strategies do you use to attend to your health needs while living in New Zealand?

6) What matters most when you access health care services? (It can be English proficiency, cost, knowledge of health care system, age, gender, ethnicity, cultural factors, and personal belief.)

7) What kind of healthcare do you seek?

These are the preliminary study questions. These will be modified as the study progresses. 


\section{Appendix 7. Demographics of participants}

\begin{tabular}{|c|c|c|c|c|c|c|c|}
\hline \multirow{2}{*}{ ID } & \multirow{2}{*}{$\begin{array}{l}\text { Gender/ } \\
\text { Age }\end{array}$} & \multirow{2}{*}{$\begin{array}{c}\text { Duration } \\
\text { (yrs) }\end{array}$} & \multirow{2}{*}{ Employment } & \multirow{2}{*}{$\begin{array}{l}\text { Marital status and } \\
\text { family members }\end{array}$} & \multirow{2}{*}{ Healthcare experience in $\mathrm{NZ}$} & \multicolumn{2}{|c|}{ Healthcare experience in Korea } \\
\hline & & & & & & Before came to $\mathrm{NZ}$ & After came to $\mathrm{NZ}$ \\
\hline IN01 & F56 & 15 & Full-time & Divorced; 1 son & GP service, screenings & Specialist treatment & None \\
\hline IN02 & F57 & 20 & Part-time & $\begin{array}{l}\text { Married; husband, } \\
2 \text { daughters }\end{array}$ & $\begin{array}{l}\text { GP service, ED service, public } \\
\text { hospital care, screenings }\end{array}$ & Specialist treatment & Medical check-ups \\
\hline IN03 & M47 & 15 & Full-time & $\begin{array}{l}\text { Married; wife, } \\
1 \text { daughter }\end{array}$ & $\begin{array}{l}\text { GP service, ED service, public } \\
\text { hospital care, screenings }\end{array}$ & Specialist treatment & $\begin{array}{l}\text { Medical check-ups, dental } \\
\text { care }\end{array}$ \\
\hline IN04 & M37 & 7 & Own business & $\begin{array}{l}\text { Married; wife, } \\
2 \text { daughters }\end{array}$ & $\begin{array}{l}\text { GP service, ED service, public } \\
\text { hospital care }\end{array}$ & None & None \\
\hline IN05 & M47 & 19 & Full-time & $\begin{array}{l}\text { Married; wife, } \\
1 \text { daughter }\end{array}$ & $\begin{array}{l}\text { GP service, ED service, private } \\
\text { care, complementary care } \\
\text { (acupuncture, chiropractic) }\end{array}$ & Specialist treatment & $\begin{array}{l}\text { Specialist treatment } \\
\text { (surgery), medical check- } \\
\text { ups }\end{array}$ \\
\hline IN06 & M54 & 15 & Own business & $\begin{array}{l}\text { Married; wife, } \\
1 \text { daughter }\end{array}$ & $\begin{array}{l}\text { GP service, ED service, public } \\
\text { hospital care, screenings }\end{array}$ & Specialist treatment & None \\
\hline IN07 & F52 & 22 & Part-time & $\begin{array}{l}\text { Married; husband, } \\
1 \text { son, } 1 \text { daughter }\end{array}$ & $\begin{array}{l}\text { GP service, ED service, public } \\
\text { hospital care, private care, } \\
\text { screenings }\end{array}$ & Specialist treatment & None \\
\hline IN08 & F53 & 17 & Unemployed & $\begin{array}{l}\text { Married; husband, } \\
1 \text { son }\end{array}$ & $\begin{array}{l}\text { GP service, ED service, public } \\
\text { hospital care, private care, } \\
\text { screenings }\end{array}$ & Specialist treatment & $\begin{array}{l}\text { Specialist treatment } \\
\text { (surgery), medical check- } \\
\text { ups }\end{array}$ \\
\hline IN09 & M56 & 21 & Full-time & Married Couple; & $\begin{array}{l}\text { GP service, ED service, public } \\
\text { hospital care, private care, }\end{array}$ & $\begin{array}{l}\text { Specialist treatment, } \\
\text { medical check-ups, dental } \\
\text { care }\end{array}$ & Dental care \\
\hline IN10 & F52 & 21 & Full-time & 1 son, 1 daughter & $\begin{array}{l}\text { complementary care (acupuncture, } \\
\text { chiropractic) }\end{array}$ & $\begin{array}{l}\text { Specialist treatment, } \\
\text { medical check-ups, dental } \\
\text { care }\end{array}$ & $\begin{array}{l}\text { Medical check-ups, dental } \\
\text { care }\end{array}$ \\
\hline IN11 & M40 & 4 & Own business & Married; wife & $\begin{array}{l}\text { GP service, public hospital care, } \\
\text { screenings }\end{array}$ & Specialist treatment & $\begin{array}{l}\text { Medical check-ups, dental } \\
\text { care }\end{array}$ \\
\hline
\end{tabular}




\begin{tabular}{|c|c|c|c|c|c|c|c|}
\hline \multirow{2}{*}{ ID } & \multirow{2}{*}{$\begin{array}{l}\text { Gender/ } \\
\text { Age }\end{array}$} & \multirow{2}{*}{$\begin{array}{c}\text { Duration } \\
\text { (yrs) }\end{array}$} & \multirow{2}{*}{ Employment } & \multirow{2}{*}{$\begin{array}{l}\text { Marital status and } \\
\text { family members }\end{array}$} & \multirow{2}{*}{ Healthcare experience in NZ } & \multicolumn{2}{|c|}{ Healthcare experience in Korea } \\
\hline & & & & & & Before came to NZ & After came to $\mathrm{NZ}$ \\
\hline IN12 & F63 & 4 & Home-maker & $\begin{array}{l}\text { Married; husband } \\
\text { (in Korea), } 1 \text { son }\end{array}$ & GP service, screenings & $\begin{array}{l}\text { Specialist treatment } \\
\text { (surgery), medical check- } \\
\text { ups, dental care }\end{array}$ & $\begin{array}{l}\text { Specialist treatment, } \\
\text { medical check-ups, dental } \\
\text { care }\end{array}$ \\
\hline IN13 & F37 & 10 & Home-maker & $\begin{array}{l}\text { Married; husband, } \\
3 \text { sons }\end{array}$ & $\begin{array}{l}\text { GP service, ED service, public } \\
\text { hospital care, dental care, } \\
\text { screenings }\end{array}$ & Specialist treatment & Dental care \\
\hline IN14 & F43 & 11 & Home-maker & $\begin{array}{l}\text { Married; husband, } \\
1 \text { son }\end{array}$ & $\begin{array}{l}\text { GP service, ED service, public } \\
\text { hospital care, private care, dental } \\
\text { care }\end{array}$ & Specialist treatment & $\begin{array}{l}\text { Medical check-ups, dental } \\
\text { care }\end{array}$ \\
\hline IN15 & F57 & 21 & Home-maker & Married; husband & $\begin{array}{l}\text { GP service, public hospital care, } \\
\text { private care, screening }\end{array}$ & Specialist treatment & $\begin{array}{l}\text { Specialist treatment } \\
\text { (surgery), medical check- } \\
\text { ups, dental care }\end{array}$ \\
\hline IN16 & F33 & 5 & Home-maker & $\begin{array}{l}\text { Married; husband, } \\
\text { husband's parents }\end{array}$ & $\begin{array}{l}\text { GP service, ED service, public } \\
\text { hospital care }\end{array}$ & $\begin{array}{l}\text { Specialist treatment, } \\
\text { medical check-ups, dental } \\
\text { care }\end{array}$ & $\begin{array}{l}\text { Specialist treatment } \\
\text { (surgery), medical check- } \\
\text { ups, dental care }\end{array}$ \\
\hline IN17 & F61 & 21 & Home-maker & Married; husband & $\begin{array}{l}\text { GP service, ED service, public } \\
\text { hospital care, private care, } \\
\text { screenings }\end{array}$ & Specialist treatment & $\begin{array}{l}\text { Specialist treatment } \\
\text { (surgery), medical check- } \\
\text { ups }\end{array}$ \\
\hline IN18 & F49 & 15 & Own business & Married & $\begin{array}{l}\text { GP service, ED service, public } \\
\text { hospital care, screenings }\end{array}$ & $\begin{array}{l}\text { Specialist treatment } \\
\text { (surgery) }\end{array}$ & None \\
\hline IN19 & F36 & 4 & Part-time & $\begin{array}{l}\text { Married; husband, } \\
\text { husband's parents }\end{array}$ & GP service, screening & Specialist treatment & None \\
\hline IN20 & F53 & 17 & Full-time & $\begin{array}{l}\text { Married; husband, } \\
1 \text { son }\end{array}$ & $\begin{array}{l}\text { GP service, ED service, public } \\
\text { hospital care, private care, } \\
\text { screenings }\end{array}$ & Specialist treatment & $\begin{array}{l}\text { Specialist treatment, } \\
\text { dental care }\end{array}$ \\
\hline IN21 & F39 & 6 & Part-time & Married; husband & $\begin{array}{l}\text { GP service, ED service, public } \\
\text { hospital care, private care, } \\
\text { screenings }\end{array}$ & Specialist treatment & $\begin{array}{l}\text { Specialist treatment } \\
\text { (husband' surgery) }\end{array}$ \\
\hline
\end{tabular}




\begin{tabular}{|c|c|c|c|c|c|c|c|c|}
\hline \multicolumn{2}{|c|}{ IN22 } & M40 & 8 & Own business & $\begin{array}{l}\text { Married; wife, } \\
\text { mother, } 2 \text { daughters, } \\
1 \text { son }\end{array}$ & $\begin{array}{l}\text { GP service, public hospital care, } \\
\text { screenings }\end{array}$ & $\begin{array}{l}\text { Specialist treatment } \\
\text { (surgery), medical check- } \\
\text { ups, dental care }\end{array}$ & None \\
\hline \multicolumn{2}{|c|}{ IN23 } & $\mathrm{F} 49$ & 16 & Full-time & $\begin{array}{l}\text { Married; husband, } \\
2 \text { daughters }\end{array}$ & $\begin{array}{l}\text { GP service, ED service, public } \\
\text { hospital care, screenings }\end{array}$ & Specialist treatment & None \\
\hline \multirow[t]{2}{*}{ T01 } & $\mathrm{a}$ & M38 & 6 & Full-time & \multirow[t]{2}{*}{ Married couple } & \multirow{2}{*}{$\begin{array}{l}\text { GP service, ED service, public } \\
\text { hospital care, private care, } \\
\text { screenings }\end{array}$} & None & $\begin{array}{l}\text { Specialist treatment } \\
\text { (surgery), medical check- } \\
\text { ups }\end{array}$ \\
\hline & $\mathrm{b}$ & \multicolumn{3}{|c|}{ See IN21 } & & & & \\
\hline \multirow{2}{*}{ T02 } & $\mathrm{a}$ & F50 & 23 & Full-time & $\begin{array}{l}\text { Married; husband } \\
\text { (in Korea), } 1 \text { son }\end{array}$ & $\begin{array}{l}\text { GP service, ED service, public } \\
\text { hospital care, screenings }\end{array}$ & Specialist treatment & $\begin{array}{l}\text { Medical check-ups, dental } \\
\text { care }\end{array}$ \\
\hline & $\mathrm{b}$ & F57 & 25 & Own business & $\begin{array}{l}\text { Married; husband, } \\
1 \text { daughter, } 1 \text { son }\end{array}$ & $\begin{array}{l}\text { GP service, ED service, public } \\
\text { hospital care, private care, } \\
\text { screenings }\end{array}$ & $\begin{array}{l}\text { Specialist treatment } \\
\text { (surgery) }\end{array}$ & $\begin{array}{l}\text { Medical check-ups, dental } \\
\text { care }\end{array}$ \\
\hline \multirow{2}{*}{ T03 } & $\mathrm{a}$ & M46 & 7 & Own business & \multirow{2}{*}{$\begin{array}{l}\text { Married couple; } 1 \\
\text { son }\end{array}$} & \multirow{2}{*}{$\begin{array}{l}\text { GP service, ED service, public } \\
\text { hospital care }\end{array}$} & None & None \\
\hline & $\mathrm{b}$ & $\mathrm{F} 42$ & 7 & Home-maker & & & Specialist treatment & Medical check-ups \\
\hline \multirow{2}{*}{ T04 } & $\mathrm{a}$ & M56 & 11 & Own business & \multirow{2}{*}{$\begin{array}{l}\text { Married couple; } \\
2 \text { daughters }\end{array}$} & \multirow{2}{*}{$\begin{array}{l}\text { GP service, ED service, public } \\
\text { hospital care, private care, } \\
\text { screenings }\end{array}$} & $\begin{array}{l}\text { Specialist treatment, } \\
\text { medical check-ups, dental } \\
\text { care }\end{array}$ & $\begin{array}{l}\text { Medical check-ups, dental } \\
\text { care }\end{array}$ \\
\hline & $\mathrm{b}$ & F57 & 11 & Own business & & & $\begin{array}{l}\text { Specialist treatment } \\
\text { (surgery), medical check- } \\
\text { ups, dental care }\end{array}$ & $\begin{array}{l}\text { Medical check-ups, dental } \\
\text { care }\end{array}$ \\
\hline \multirow[t]{2}{*}{ T05 } & $\mathrm{a}$ & F55 & 14 & Home-maker & $\begin{array}{l}\text { Married; husband, } \\
1 \text { son }\end{array}$ & $\begin{array}{l}\text { GP service, ED service, private } \\
\text { care, screenings }\end{array}$ & $\begin{array}{l}\text { Specialist treatment, dental } \\
\text { care }\end{array}$ & $\begin{array}{l}\text { Specialist treatment } \\
\text { (surgery), medical check- } \\
\text { ups, dental care }\end{array}$ \\
\hline & $\mathrm{b}$ & \multicolumn{7}{|c|}{ See IN01 } \\
\hline
\end{tabular}




\begin{tabular}{|c|c|c|c|c|c|c|c|c|}
\hline \multirow{2}{*}{\multicolumn{2}{|c|}{ ID }} & \multirow{2}{*}{$\begin{array}{l}\text { Gender/ } \\
\text { Age }\end{array}$} & \multirow{2}{*}{$\begin{array}{l}\text { Duration } \\
\quad(\text { yrs })\end{array}$} & \multirow{2}{*}{ Employment } & \multirow{2}{*}{$\begin{array}{l}\text { Marital status and } \\
\text { family members }\end{array}$} & \multirow{2}{*}{ Healthcare experience in $\mathrm{NZ}$} & \multicolumn{2}{|c|}{ Healthcare experience in Korea } \\
\hline & & & & & & & Before came to $\mathrm{NZ}$ & Before came to $\mathrm{NZ}$ \\
\hline \multirow{2}{*}{ T06 } & a & M45 & 16 & Full-time & \multirow{2}{*}{$\begin{array}{l}\text { Married couple; } \\
1 \text { son, } 1 \text { daughter }\end{array}$} & \multirow{2}{*}{$\begin{array}{l}\text { GP service, ED service, public } \\
\text { hospital care, private care, } \\
\text { screenings, complementary care } \\
\text { (acupuncture) }\end{array}$} & $\begin{array}{l}\text { Specialist treatment, } \\
\text { medical check-ups, dental } \\
\text { care }\end{array}$ & $\begin{array}{l}\text { Medical check-ups, dental } \\
\text { care }\end{array}$ \\
\hline & $\mathrm{b}$ & $\mathrm{F} 44$ & 16 & Home-maker & & & $\begin{array}{l}\text { Specialist treatment, } \\
\text { medical check-ups, dental } \\
\text { care }\end{array}$ & $\begin{array}{l}\text { Medical check-ups, dental } \\
\text { care }\end{array}$ \\
\hline \multirow{2}{*}{ T07 } & $\mathrm{a}$ & M73 & 21 & Retired & \multirow{2}{*}{ Married couple } & \multirow{2}{*}{$\begin{array}{l}\text { GP service, ED service, public } \\
\text { hospital care, private care, } \\
\text { screenings }\end{array}$} & None & None \\
\hline & $\mathrm{b}$ & F66 & 21 & Retired & & & $\begin{array}{l}\text { Specialist treatment, dental } \\
\text { care }\end{array}$ & $\begin{array}{l}\text { Regular medical check- } \\
\text { ups, dental care }\end{array}$ \\
\hline \multirow{3}{*}{ G01 } & a & F56 & 14 & Home-maker & $\begin{array}{l}\text { Married; husband, } \\
2 \text { sons }\end{array}$ & $\begin{array}{l}\text { GP service, ED service, public } \\
\text { hospital care, screenings }\end{array}$ & $\begin{array}{l}\text { Specialist treatment } \\
\text { (surgery), dental care }\end{array}$ & Medical check-ups \\
\hline & $\mathrm{b}$ & F52 & 14 & Part-time & $\begin{array}{l}\text { Married; husband (in } \\
\text { Korea), } 1 \text { son, } 1 \\
\text { daughter }\end{array}$ & $\begin{array}{l}\text { GP service, ED service, public } \\
\text { hospital care, screenings }\end{array}$ & $\begin{array}{l}\text { Specialist treatment, dental } \\
\text { care }\end{array}$ & $\begin{array}{l}\text { Medical check-ups, dental } \\
\text { care }\end{array}$ \\
\hline & $\mathrm{c}$ & F61 & 4 & Part-time & $\begin{array}{l}\text { Married; husband (in } \\
\text { Korea), son and his } \\
\text { wife, } 3 \text { grandchildren }\end{array}$ & GP service & $\begin{array}{l}\text { Specialist treatment } \\
\text { (surgery), medical check- } \\
\text { ups, dental care }\end{array}$ & $\begin{array}{l}\text { Specialist treatment, } \\
\text { medical check-ups, dental } \\
\text { care }\end{array}$ \\
\hline \multirow{4}{*}{ G02 } & a & F61 & 22 & Own business & $\begin{array}{l}\text { Married; husband, } \\
2 \text { sons }\end{array}$ & $\begin{array}{l}\text { GP service, ED service, public } \\
\text { hospital care, screenings }\end{array}$ & $\begin{array}{l}\text { Specialist treatment } \\
\text { (surgery), medical check- } \\
\text { ups, dental care }\end{array}$ & $\begin{array}{l}\text { Medical check-up, dental } \\
\text { care }\end{array}$ \\
\hline & $\mathrm{b}$ & F54 & 12 & Home-maker & $\begin{array}{l}\text { Married; husband, } \\
1 \text { daughter }\end{array}$ & $\begin{array}{l}\text { GP service, ED service, public } \\
\text { hospital care, private care, } \\
\text { screenings }\end{array}$ & $\begin{array}{l}\text { Specialist treatment } \\
\text { (surgery), dental care }\end{array}$ & None \\
\hline & $\mathrm{c}$ & F44 & 11 & Home-maker & $\begin{array}{l}\text { Married; husband, } \\
1 \text { daughter }\end{array}$ & GP service & Specialist treatment & $\begin{array}{l}\text { Medical check-up, dental } \\
\text { care }\end{array}$ \\
\hline & $\mathrm{d}$ & \multicolumn{7}{|c|}{ See G01b } \\
\hline
\end{tabular}




\begin{tabular}{|c|c|c|c|c|c|c|c|c|}
\hline \multirow{4}{*}{ GO3 } & $\mathrm{a}$ & F68 & 15 & Retired & $\begin{array}{l}\text { Widow (living with } \\
\text { daughter's family) }\end{array}$ & $\begin{array}{l}\text { GP service, ED service, public } \\
\text { hospital care, private care, } \\
\text { screenings }\end{array}$ & $\begin{array}{l}\text { Specialist treatment, } \\
\text { medical check-ups, dental } \\
\text { care }\end{array}$ & $\begin{array}{l}\text { Specialist treatment, } \\
\text { medical check-ups }\end{array}$ \\
\hline & $\mathrm{b}$ & F69 & 17 & Retired & Married; husband & GP service, ED service & Specialist treatment & None \\
\hline & $\mathrm{c}$ & M57 & 14 & Unemployed & Married; wife, 2 sons & $\begin{array}{l}\text { GP service, ED service, public } \\
\text { hospital care, private care, } \\
\text { screenings }\end{array}$ & Specialist treatment & Medical check-ups \\
\hline & $\mathrm{d}$ & F71 & 11 & Retired & Widow & $\begin{array}{l}\text { GP service, ED service, public } \\
\text { hospital care, screenings }\end{array}$ & N/A & N/A \\
\hline \multirow{3}{*}{ G04 } & a & F66 & 28 & Retired & Divorced; mother & GP service, public hospital care & None & None \\
\hline & $\mathrm{b}$ & F85 & 33 & Retired & Widow; 1 daughter & $\begin{array}{l}\text { GP service, ED service, public } \\
\text { hospital care }\end{array}$ & None & None \\
\hline & $\mathrm{c}$ & F87 & 28 & Retired & Widow; alone & GP service, public hospital care & None & None \\
\hline
\end{tabular}




\section{Appendix 8. Data analysis and progress: Diagrams A, B, C}

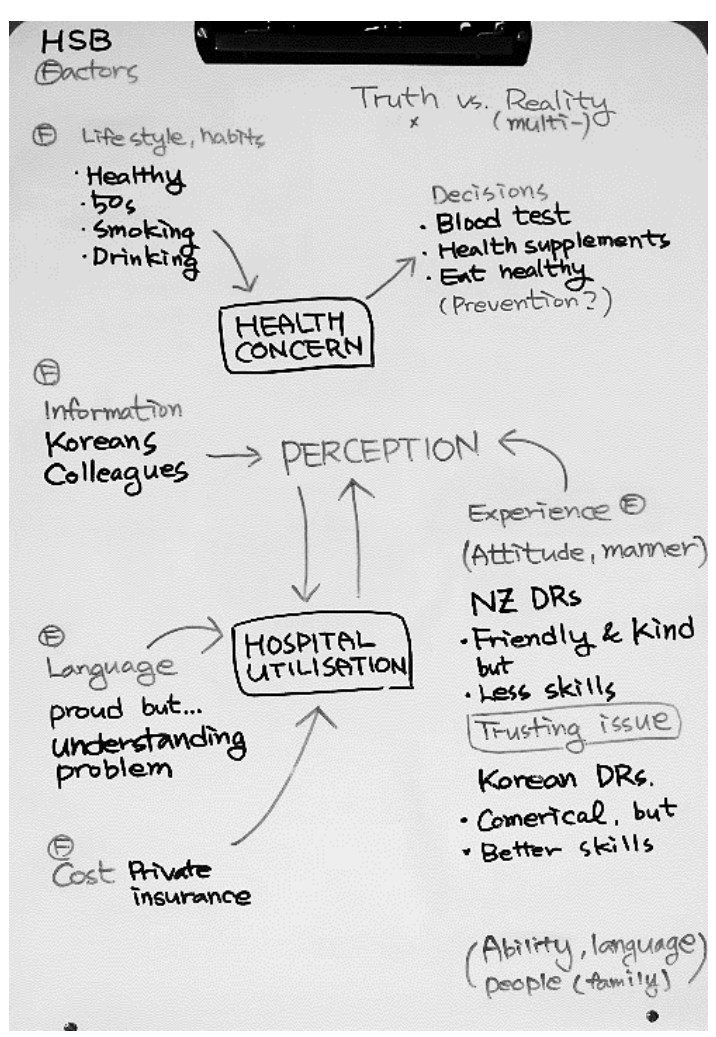

A. July 25,2014

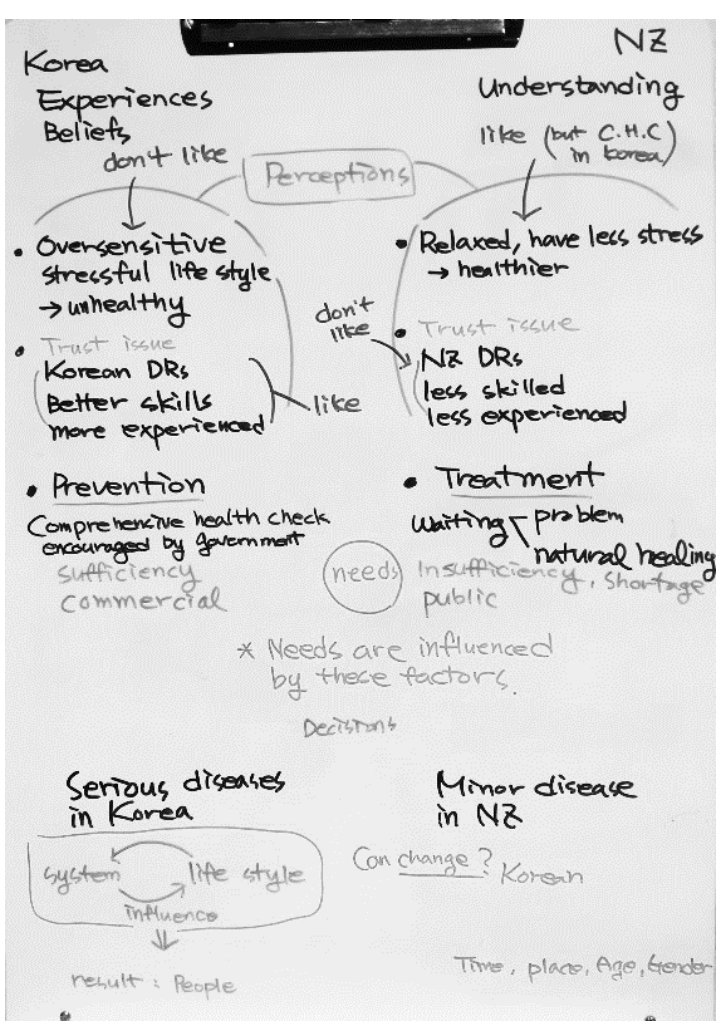

B. September 15, 2014

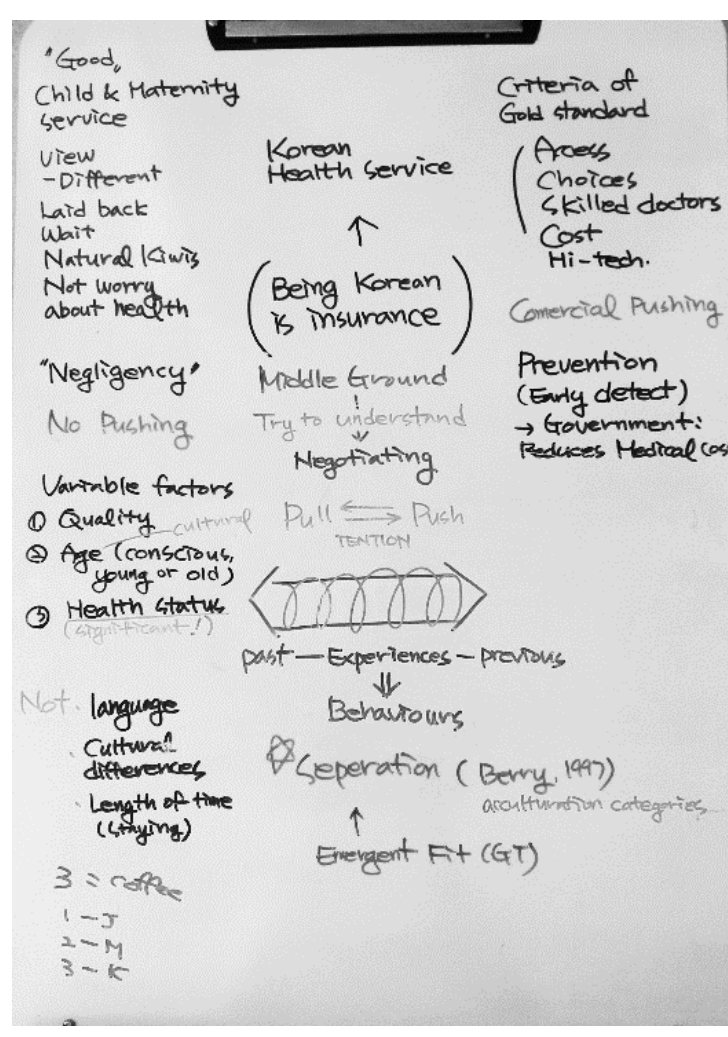

C. January 26, 2015 


\section{Appendix 9. Data analysis and progress: Diagram D}

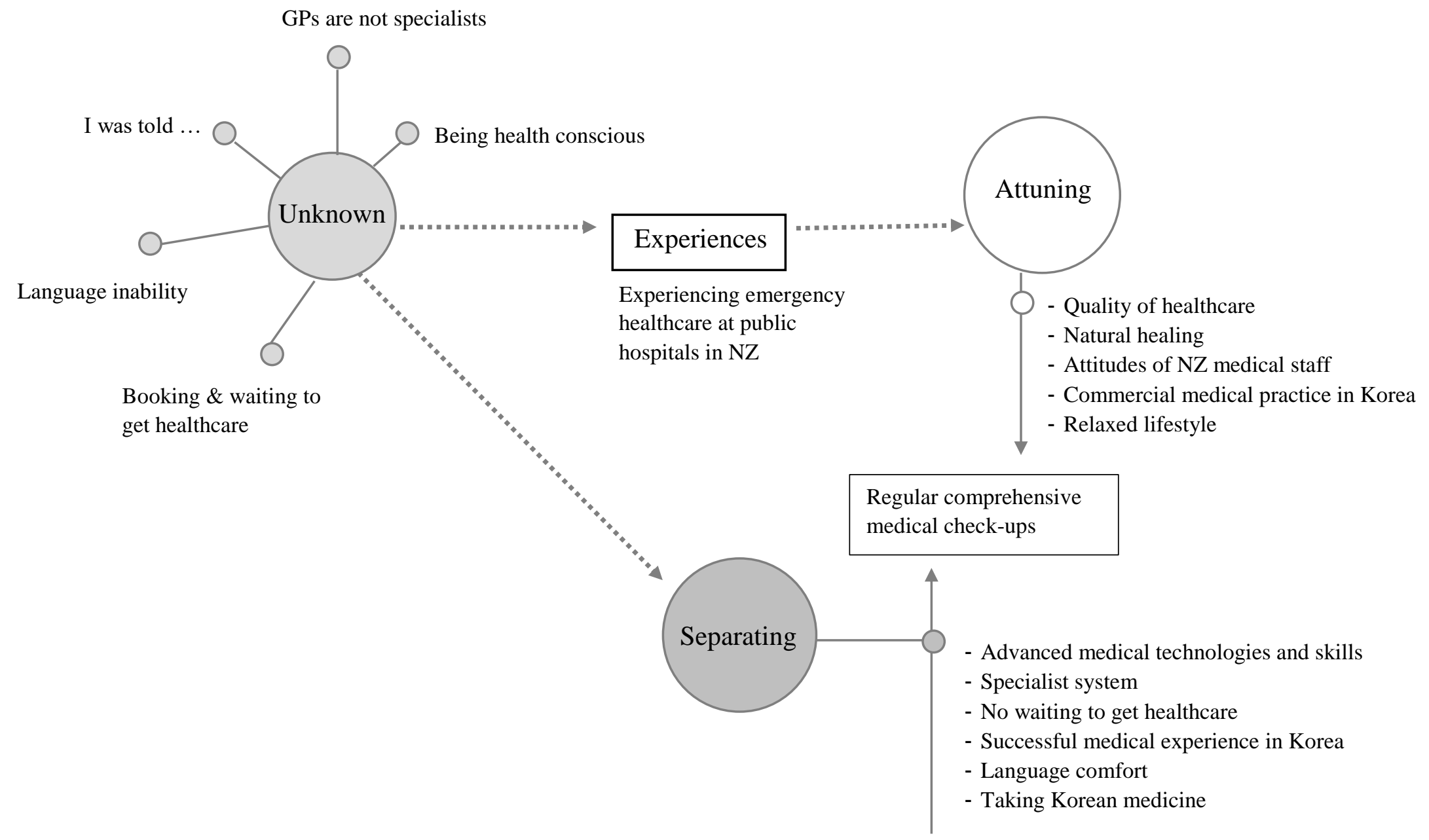

(November 28, 2014) 


\section{Appendix 10. Data analysis and progress: Diagram $\mathbf{E}$}

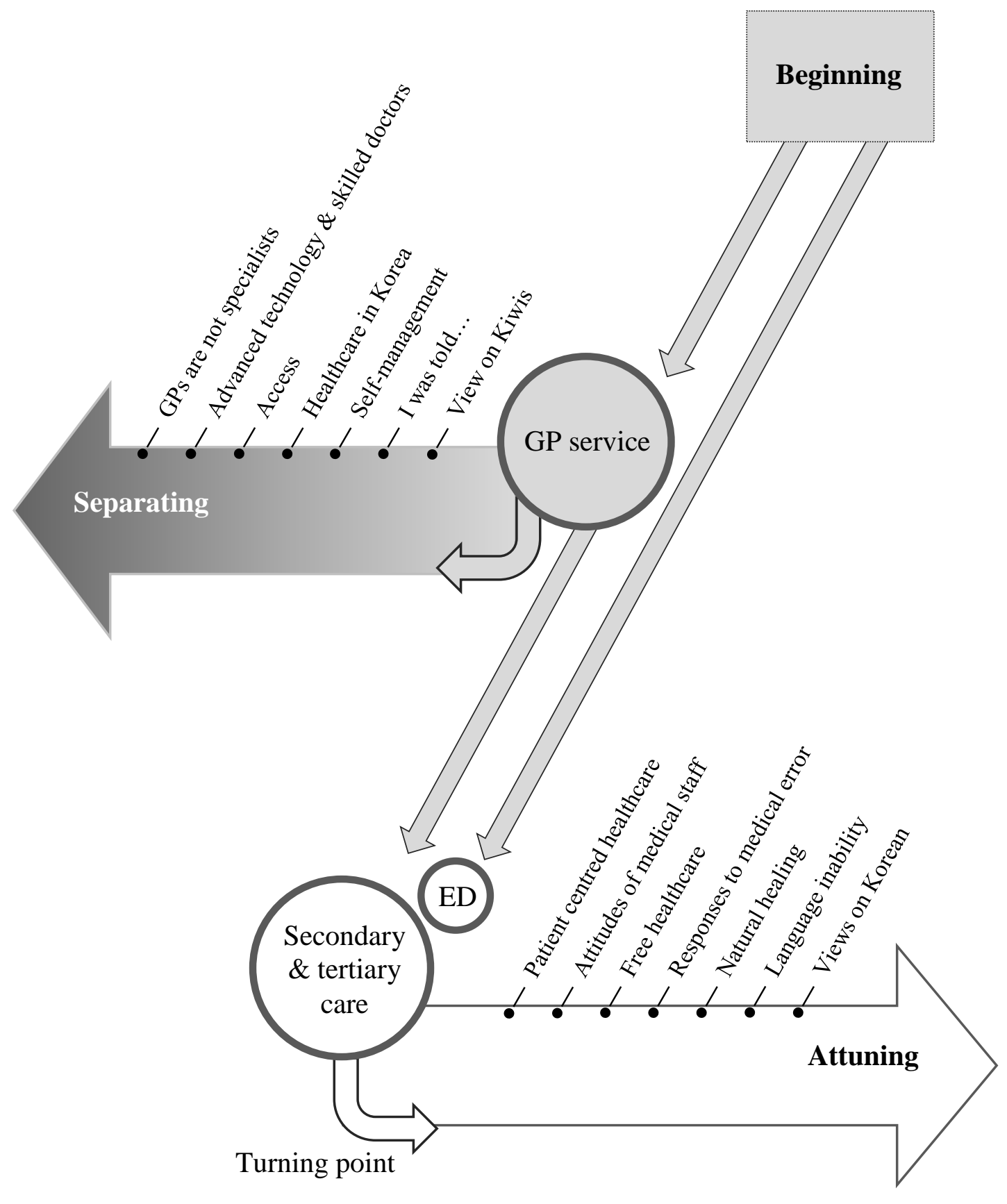

(February 12, 2015) 


\section{Appendix 11. Development of codes}

\section{Codes (data extraction)}

\section{Concepts /themes}

Categories

GPs are not specialists

A GP does all

Ineffective treatment

Want to see a specialist directly

No GP's referral, no specialist care

I was told... (negative information)

...

Skilled and experienced Korean doctors

Specialised like a conveyor belt

Convenient

Trust in specialist ...

Easy access

No booking and waiting

Quick relief

How long do I have to wait (for free care)? ...

Preventive healthcare

Relieves my anxiety

All friends of mine take

Much cheaper

I am not young anymore

No family in Korea

Cost-wise, cheaper in NZ

Cost of healthcare and travelling

I have choices

Compare care options in $\mathrm{NZ}$ and Korea

Will do if it benefits me

Diagnose accurately

Timely referrals

Listens well

Kind and friendly

\section{Care for the patient's feeling}

No time wasting

Really devoted

Care for people's lives

They do everything for me

More than medicine

Korean doctors are like businessmen

No money no hospital care in Korea

Free care but not haughty ...

Became healthier

Self-care rather than hospital care

Good for people

I have to take care of myself ver Gervice

Korea is Ideal

$\Rightarrow$ Rejecting

Fast and

effective care

with easy access

Regular

comprehensive

medical check-

ups

For the best

outcomes

(1)

For the best

outcomes

ood GP

service

Person-centred

care

Healthcare in Korea is ideal but

\section{Rejecting but negotiating}

\section{Healthcare in} New Zealand is ideal but

\section{Attuning but negotiating}

Healthcare in New Zealand is $\square$ Attuning ideal 


\section{References}

Abraido-Lanza, A. F., Chao, M. T., \& Florez, K. R. (2005). Do healthy behaviors decline with greater acculturation?: Implications for the Latino mortality paradox. Social Science \& Medicine, 61(6), 1243-1255.

Adler, P. S. (2002). Beyond cultural identity: Reflections on multiculturalism. Retrieved from Mediate website: http://www.mediate.com/articles/adler3.cfm

Akresh, I. R. (2009). Health service utilization among immigrants to the United States. Population Research and Policy Review, 28(6), 795-815.

Alegria, M., Canino, G., Shrout, P. E., Woo, M., Duan, N., Vila, D., . . Meng, X.-L. (2008). Prevalence of mental illness in immigrant and non-immigrant U.S. Latino groups. American Journal of Psychiatry, 165(3), 359-369. doi: 10.1176/appi.ajp.2007.07040704

Alexander, J. A., Hearld, L. R., Mittler, J. N., \& Harvey, J. (2012). Patient-physician role relationships and patient activation among individuals with chronic illness. Health Services Research, 47(3pt1), 1201-1223. doi: 10.1111/j.14756773.2011.01354.x

Allen, L. M. (2010). A critique of four grounded theory texts. The Qualitative Report, 15(6), 1606.

Allen, M. L., Elliott, M. N., Fuligni, A. J., Morales, L. S., Hambarsoomian, K., \& Schuster, M. A. (2008). The relationship between spanish language use and substance use behaviors among Latino youth: A social network approach. Journal of Adolescent Health, 43(4), 372-379. doi: 10.1016/j.jadohealth.2008.02.016

Alrashdi, I. (2012). Evaluation of quality of healthcare: To what extent can we rely on patient expectations and preferences. Oman Medical Journal, 27(6), 448-449. doi: 10.5001/omj.2012.107

American Psychiatric Association. (2005). Diagnostic and statistical manual of mental disorders (4th ed.). Washington, DC: Author.

American Psychological Association. (2012). Crossroads: The psychology of immigration in the new century. Retrieved from http://www.apa.org/topics/immigration/report.aspx 
American Society of Health-System Pharmacists. (2014). Drugs, herbs and supplements: Acetaminophen. Retrieved April 5, 2016, from https://www.nlm.nih.gov/medlineplus/druginfo/meds/a681004.html

Anczewska, M., Świtaj, P., Prot, K., Chrostek, A., Waszkiewicz, J., \& Indulska, A. (2011). A survey to investigate the preferred terms describing people with mental disorders-recipients' and providers' opinions. Archives of Psychiatry and Psychotherapy, 3, 25-30.

Anderson, A. (2008). Understanding migrants' primary healthcare utilisation in New Zealand through an ethnographic approach. Diversity in Health and Social Care, 5(4), 291-301.

Andreou, C. (2013). Agency and awareness. Ratio, 26(2), 117-133. doi: 10.1111/j.14679329.2012.00539.x

Andrew, T. (2006). The literature review in grounded theory: A response to McCallin (2003). The Grounded Theory Review: An International Journal, 5(2/3), 29-41.

Antecol, H., \& Bedard, K. (2006). Unhealthy assimilation: Why do immigrants converge to American health status levels? Demography, 43(2), 337-360. doi: http://link.springer.com/journal/volumesAndIssues/13524

Anwar, M., Green, J., \& Norris, P. (2012). Health-seeking behaviour in Pakistan: A narrative review of the existing literature. Public Health, 126(6), 507-517.

Arends-Tóth, J., \& Van de Vijver, F. J. (2007). Acculturation attitudes: A comparison of measurement methods. Journal of Applied Social Psychology, 37(7), 14621488.

Aroni, R., Goeman, D., Stewart, K., Sawyer, S., Abramson, M., Thien, F., \& Douglass, J. (1999). Concepts of rigour: When methodological, clinical and ethical issues intersect. Paper presented at the AQR '99 Conference, Melbourne.

Arora, S., Ashrafian, H., Davis, R., Athanasiou, T., Darzi, A., \& Sevdalis, N. (2010). Emotional intelligence in medicine: A systematic review through the context of the acgme competencies. Medical Education, 44(8), 749-764. doi: 10.1111/j.1365-2923.2010.03709.x

Asan Medical Center. (2016). 서울아산병원 종합건강진단 프로그램 [Asan medical center comprehensive health evaluation program]. Retrieved May 29, 2016, from http://health.amc.seoul.kr/health/main.do 
Auerbach, C., \& Silverstein, L. B. (2003). Qualitative data: An introduction to coding and analysis. Washington Square: New York University Press.

Ayala, G. X., Baquero, B., \& Klinger, S. (2008). A systematic review of the relationship between acculturation and diet among Latinos in the United States: Implications for future research. Journal of the American Dietetic Association, 108(8), 13301344.

Baik, T.-U., \& Murabayashi, D. H. L. (2011). Historical development of early Korean immigration to Hawaii and its legal structure. Journal of Korean Law, 11, 77100.

Baker, E., Rendall, M., \& Weden, M. (2015). Epidemiological paradox or immigrant vulnerability? Obesity among young children of immigrants. Demography, 52(4), 1295-1320. doi: 10.1007/s13524-015-0404-3

Ballara, A. (1986). Proud to be white. Auckland: Heinemann Publishers.

Barnardos. (2016, April 7). Barnardos welcomes recommendations on care of vulnerable children. from http://www.barnardos.org.nz/news

Batchelor, J. A., \& Briggs, C. M. (1994). Subject, project or self? Thoughts on ethical dilemmas for social and medical researchers. Social Science \& Medicine, 39(7), 949-954. doi: http://dx.doi.org/10.1016/0277-9536(94)90206-2

Batson, C. D. (2002). Addressing the altruism question experimentally. In S. G. Post, L. G. Underwood, J. Schloss, \& W. B. Hurlbut (Eds.), Altruism and altruistic love : Science, philosophy, and religion in dialogue (pp. 89-105). Cary, NC, USA: Oxford University Press.

Baycan, T., \& Nijkamp, P. (2013). The migration-development nexus: New perspectives and challenges. Working Paper. Vrije Universiteit, Faculteit der Economische Wetenschappen en Bedrijskunde. Amsterdam. Retrieved from http://hdl.handle.net/1871/39794

Beasley, R., Bibby, S., \& Weatherall, M. (2008). Leukotriene receptor antagonist therapy and Churg-Strauss Syndrome: Culprit or innocent bystander? Thorax, 63(10), 847-849. doi: 10.1136/thx.2007.093971

Becker, G., Gates, R. J., \& Newsom, E. (2004). Self-care among chronically ill African Americans: Culture, health disparities, and health insurance status. American Journal of Public Health, 94(12), 2066-2073. 
Benet-Martínez, V., Leu, J., Lee, F., \& Morris, M. W. (2002). Negotiating biculturalism cultural frame switching in biculturals with oppositional versus compatible cultural identities. Journal of Cross-Cultural Psychology, 33(5), 492-516.

Berger, S., Braehler, E., \& Ernst, J. (2012). The health professional-patient-relationship in conventional versus complementary and alternative medicine. A qualitative study comparing the perceived use of medical shared decision-making between two different approaches of medicine. Patient Education and Counseling, 88(1), 129-137.

Berry, J. W. (1997). Immigration, acculturation, and adaptation. Applied Psychology, 46(1), 5-34. doi: 10.1111/j.1464-0597.1997.tb01087.x

Berry, J. W. (2003). Conceptual approaches to acculturation. In K. M. Chun, P. Balls Organista, \& G. Marín (Eds.), Acculturation: Advances in theory, measurement, and applied research (pp. 17-37). Washington, DC: American Psychological Association.

Berry, J. W. (2004). Fundamental psychological processes in intercultural relations. In D. Landis, J. Bennett, \& M. Bennett (Eds.), Handbook of intercultural training (pp. 166-185). Thousand Oaks, CA: SAGE Publications.

Berry, J. W. (2005). Acculturation: Living successfully in two cultures. International Journal of Intercultural Relations, 29(6), 697-712. doi: 10.1016/j.ijintrel.2005.07.013

Berry, J. W. (2006). Acculturative stress. In P. P. Wong \& L. J. Wong (Eds.), Handbook of multicultural perspectives on stress and coping (pp. 287-298). British Columbis: Springer US.

Berry, J. W., Kim, U., Minde, T., \& Mok, D. (1987). Comparative studies of acculturative stress. International Migration Review, 21(3), 491-511. doi: $10.2307 / 2546607$

Berry, J. W., Phinney, J. S., Sam, D. L., \& Vedder, P. (2006). Immigrant youth: Acculturation, identity, and adaptation. Applied Psychology, 55(3), 303-332.

Berterö, C. (2012). Grounded theory methodology - has it become a movement? International Journal of Qualitative Studies on Health and Well-being, 7, 10.3402/qhw.v3407i3400.18571. doi: 10.3402/qhw.v7i0.18571 
Berwick, D. M. (2009). What 'patient-centered' should mean: Confessions of an extremist. Health Affairs, 28(4), w555-w565. doi: 10.1377/hlthaff.28.4.w555

Bhatia, S., \& Ram, A. (2001). Rethinking 'acculturation'in relation to diasporic cultures and postcolonial identities. Human Development, 44(1), 1-18.

Bijak, J. (2006). Forecasting international migration: Selected theories, models, and methods. CEFMR Working Paper 4/2006. Central European Forum for Migration Research in Warsaw. Retrieved from http://www.ppge.ufrgs.br/giacomo/arquivos/eco02268/bijak-2006.pdf

Birks, M., \& Mills, J. (2010). Grounded theory: A practical guide. Thousand Oaks, California: SAGE Publications.

Bland, J. D. P. (2007). Carpal tunnel syndrome. BMJ, 335(7615), 343-346. doi: 10.1136/bmj.39282.623553.AD

Blank, K. (2010). Health care consumerism. Employee Benefit Plan Review, 64(8), 9.

Bluff, R. (2005). Grounded theory: The methodology. In H. Immy (Ed.), Qualitative research in health care (pp. 147-167). London: Open University Press.

Blumer, H. (1986). Symbolic interactionism: Perspective and method. Los Angeles: University of California Press.

Blundell, N., Taylor-Phillips, S., Spitzer, D., Martin, S., Forde, I., \& Clarke, A. (2011). Elective surgical referral guidelines-background educational material or essential shared decision making tool? A survey of GPs' in England. BMC Family Practice, 12(1), 92.

Bodvarsson, Ö., \& Van den Berg, H. (2013). The determinants of international migration: Theory. The economics of immigration (2nd ed., pp. 27-57). New York: Springer.

Borjas, G. J. (1989). Economic theory and international migration. International Migration Review, 23(3), 457-485.

Boruchovitch, E., \& Mednick, B. R. (2002). The meaning of health and illness: Some considerations for health psychology. Psico-USF, 7(2), 175-183. Retrieved from: http://www.scielo.br/pdf/pusf/v7n2/v7n2a06.pdf

Bowling, A., Rowe, G., Lambert, N., Waddington, M., Mahtani, K., Kenten, C., . . . Francis, S. (2012). The measurement of patients' expectations for health care: A 
review and psychometric testing of a measure of patients' expectations. Health Technology Assessment 16(30). doi: http://dx.doi.org/10.3310/hta16300

Bowling, A., Rowe, G., \& McKee, M. (2013). Patients' experiences of their healthcare in relation to their expectations and satisfaction: A population survey. Journal of the Royal Society of Medicine, 106(4), 143-149. doi: 10.1258/jrsm.2012.120147

Bozic-Vrbancic, S. (2003). One Nation, Two Peoples, Many Cultures: Exhibiting Identity at Te Papa Tongarewa. Journal of the Polynesian Society, 112(3), 295313.

http://www.jps.auckland.ac.nz/docs/Volume112/jps_v116_no3_2003/6\%20One \%20nation.pdf

Breyer, D. S. (2013). Ownership, agency, and defeat. Acta Analytica, 28(2), 253-256. doi: 10.1007/s12136-012-0175-Z

Brown, S. W. (1993). Patient satisfaction pays: Quality service for practice success. Gaithersburg, Maryland: Aspen Publisher, Inc.

Brunner, S. (2015). Pulmonary edema: Causes, symptoms and treatment. from www.medicalnewstoday.com/articles/167533.php

Bryant, A., \& Charmaz, K. (2010). Grounded theory in historical perspective: An epistemological account. In A. Bryant \& K. Charmaz (Eds.), The Sage handbook of grounded theory: Paperback edition (pp. 31-57). Los Angeles, London: SAGE Publications.

Bryman, A. (2008). Social research methods (3rd ed.). New York: Oxford University Press.

Bulawa, P. (2014). Adapting grounded theory in qualitative research: Reflections from personal experience. International Research in Education, 2(1), 145-168.

Burks, D. J., Youll, L. K., \& Durtschi, J. P. (2012). The empathy-altruism association and its relevance to health care professions. Social Behavior and Personality: An International Journal, 40(3), 395-400.

Cain, T., \& Spoonley, P. (2013). Making it work: The mixed embeddedness of immigrant entrepreneurs in New Zealand. IZA DP No. 7332. Retrieved from https://www.massey.ac.nz/massey/fms/Colleges/College\%20of\%20Business/nto m/Cain\%20and\%20Spoonley\%202013.pdf 
Calabrese, C. (2012). Textbook of natural medicine. In J. E. Pizzorno \& M. T. Murray (Eds.), (pp. 93-100). United States of America: Elsevier Health Sciences.

Carrasquillo, O., Carrasquillo, A. I., \& Shea, S. (2000). Health insurance coverage of immigrants living in the United States: Differences by citizenship status and country of origin. American Journal of Public Health, 90(6), 917-923.

Carroll, L., \& Qiu, L. (2015, November 19). Politifact sheet: 5 questions about Syrian refugees. PolitiFact. Retrieved from http://www.politifact.com/truth-ometer/article/2013/nov/01/principles-politifact-punditfact-and-truth-o-meter/

Castles, S. (2000). International migration at the beginining of the twenty-first century: Global trends and issues. International Social Science Journal, 52(165), 269281. doi: $10.1111 / 1468-2451.00258$

Castles, S. (2010). Understanding global migration: A social transformation perspective. Journal of Ethnic and Migration Studies, 36(10), 1565-1586.

Castles, S., \& Miller, M. J. (2009). The age of migration: International population movements in the modern world (4th ed.). New York and London: Palgrave Macmillan Limited.

Chambliss, D. F., \& Schutt, R. K. (2012). Making sense of the social world: Methods of investigation. Thousand Oaks: SAGE Publications.

Chang, S., Morris, C., \& Vokes, R. W. A. (2006). Korean migrant families in Christchurch: Expectations and experiences. Wellington, New Zealand: Families Commission Retrieved from http://www.superu.govt.nz/sites/default/files/BS-korean-migrant-families.pdf.

Charmaz, K. (1990). 'Discovering' chronic illness: Using grounded theory. Social Science \& Medicine, 30(11), 1161-1172. doi: http://dx.doi.org/10.1016/02779536(90)90256-R

Charmaz, K. (2000). Grounded theory: Objectivist and constructivist methods. In N. K. Denzin \& Y. S. Lincoln (Eds.), The SAGE handbook of qualitative research (2nd ed., pp. 509-535). Thousand Oaks, Ca.: SAGE Publications.

Charmaz, K. (2006). Measuring pursuits, marking self: Meaning construction in chronic illness. International Journal of Qualitative Studies on Health and Well-Being, 1(1), 27-37. doi: 10.1080/17482620500534488 
Charmaz, K. (2014). Constructing grounded theory (2nd ed.). Thousand Oaks, Ca: SAGE Publications.

Charmaz, K., \& Henwood, K. (2008). Grounded theory. In C. Willig \& W. StaintonRogers (Eds.), The Sage handbook of qualitative research in psychology (pp. 240-262). London, England: SAGE Publications.

Chen, H. Y., \& Boore, J. R. (2009). Using a synthesised technique for grounded theory in nursing research. Journal of Clinical Nursing, 18(16), 2251-2260.

Chen, J. (2011). Internal migration and health: Re-examining the healthy migrant phenomenon in China. Social Science \& Medicine, 72(8), 1294-1301.

Chen, S. X., Benet-Martínez, V., \& Harris Bond, M. (2008). Bicultural identity, bilingualism, and psychological adjustment in multicultural societies: Immigration-based and globalization-based acculturation. Journal of Personality, 76(4), 803-838.

Chirkov, V. (2009). Summary of the criticism and of the potential ways to improve acculturation psychology. International Journal of Intercultural Relations, 33(2), 177-180. doi: 10.1016/j.ijintrel.2009.03.005

Cho, J. Y., \& Lee, E.-H. (2014). Reducing confusion about grounded theory and qualitative content analysis: Similarities and differences. The Qualitative Report, 19(32), 1-20.

Choi, J. Y. (2009). Contextual effects on health care access among immigrants: Lessons from three ethnic communities in Hawaii. Social Science \& Medicine, 69(8), 1261-1271.

Choi, J. Y. (2013a). Negotiating old and new ways: Contextualizing adapted health care-seeking behaviors of Korean immigrants in Hawaii. Ethnicity \& Health, 18(4), 350-366. doi: 10.1080/13557858.2012.734280

Choi, J. Y. (2013b). Reconstruction of health-seeking behaviors: A comparative study of three Asian Pacific immigrant groups. Qualitative Health Research, 23(4), 517-530. doi: 10.1177/1049732312469731

Choi, K.-H., Park, S.-M., Lee, J.-H., \& Kwon, S. (2012). Factors affecting the prescribing patterns of antibiotics and injections. Journal of Korean Medical Science, 27(2), 120-127. 
Choi, K.-S., Kim, C., Lee, S., Lee, H., \& Cho, W.-H. (2004). The relationships among quality, value, satisfaction and behavioral intention in health care provider choice: A South Korean study. Journal of Business Research, 57(8), 913-921. doi: 10.1016/S0148-2963(02)00293-X

Choi, M., \& Yeom, H.-A. (2011). Identifying and treating the culture-bound syndrome of hwa-byung among older Korean immigrant women: Recommendations for practitioners. Journal of the American Academy of Nurse Practitioners, 23(5), 226-232 227p. doi: 10.1111/j.1745-7599.2011.00607.x

Chow, A., Mayer, E. K., Darzi, A. W., \& Athanasiou, T. (2009). Patient-reported outcome measures: The importance of patient satisfaction in surgery. Surgery, 146(3), 435-443. doi: http://dx.doi.org/10.1016/j.surg.2009.03.019

Chun, C. B., Kim, S. Y., Lee, J. Y., \& Lee, S. Y. (2009). Republic of Korea: Health system review. Health Systems in Transition, 11(7), 1-184.

Chun, H., \& Mobley, M. (2014). The "immigrant paradox" phenomenon: Assessing problem behaviors and risk factors among immigrant and native adolescents. Journal of Primary Prevention, 35(5), 339-356. doi: 10.1007/s10935-014-0359$\mathrm{y}$

Chung, B. Y., Xu, Y., \& Kwak, C. (2005). Hospice and hospice care in Korea: Evolution, current status, and challenges. Home Health Care Management \& Practice, 18(1), 73-77. doi: 10.1177/1084822305279830

Clarke, A. E. (2005). Situational analysis. Situational analysis. Thousand Oaks, CA: SAGE Publications, Inc.

Clegg, A. (2003). Older South Asian patient and carer perceptions of culturally sensitive care in a community hospital setting. Journal of Clinical Nursing, 12(2), 283290. doi: 10.1046/j.1365-2702.2003.00724.x

Clough, J., Lee, S., \& Chae, D. H. (2013). Barriers to health care among Asian immigrants in the United States: A traditional review. Journal of Health Care for the Poor and Underserved, 24(1), 384-403.

Conrad, P., \& Barker, K. K. (2010). The social construction of illness: Key insights and policy implications. Journal of Health and Social Behavior, 51(1), S67-S79. doi: $10.1177 / 0022146510383495$ 
Corbin, J., \& Strauss, A. (2008). Basics of qualitative research: Techniques and procedures for developing grounded theory (3rd ed.). Los Angeles, London: SAGE Publications.

Corbin, J., \& Strauss, A. (2014). Basics of qualitative research: Techniques and procedures for developing grounded theory (4th ed.). Los Angeles, London: SAGE Publications.

Corlin, L., Woodin, M., Thanikachalam, M., Lowe, L., \& Brugge, D. (2014). Evidence for the healthy immigrant effect in older Chinese immigrants: A cross-sectional study. BMC Public Health, 14(1), 603.

Costigan, C., \& Su, T. (2004). Orthogonal versus linear models of acculturation among immigrant Chinese Canadians: A comparison of mothers, fathers, and children. International Journal of Behavioral Development, 28(6), 518-527.

Crampton, P., Jatrana, S., Lay-Yee, R., \& Davis, P. (2007). Exposure to primary medical care in New Zealand: Number and duration of general practitioner visits. The New Zealand Medical Journal (Online), 120(1256).

Creswell, J. W. (2012). Qualitative inquiry and research design: Choosing among five approaches (3rd ed.). Thousand Oaks, CA.: SAGE Publications, Inc.

Crooks, D. L. (2001). The importance of symbolic interaction in grounded theory research on women's health. Health Care for Women International, 22(1-2), 1127.

Cruess, R. L., \& Cruess, S. R. (2008). Expectations and obligations: Professionalism and medicine's social contract with society. Perspectives in Biology and Medicine, 51(4), 579-598.

Cruess, S. R., Cruess, R. L., \& Johnston, S. (2004). Professionalism for medicine: Opportunities and obligations. The Iowa Orthopaedic Journal, 24, 9-15.

Cumming, J., McDonald, J., Barr, C., Martin, J., Gerring, Z., \& Daubé, J. (2014). Health systems in transition: New Zealand health system review. Wellington: WHO Regional Office for the Western Pacific Retrieved from http://www.wpro.who.int/asia_pacific_observatory/hits/series/Nez_Health_Syste ms_Review.pdf.

Cunningham, S. A., Ruben, J. D., \& Narayan, K. V. (2008). Health of foreign-born people in the United States: A review. Health \& Place, 14(4), 623-635. 
Czaika, M., \& de Haas, H. (2014). The globalization of migration: Has the world become more migratory? International Migration Review, 48(2), 283-323. doi: 10.1111/imre.12095

Dastjerdi, M., Olson, K., \& Ogilvie, L. (2012). A study of iranian immigrants' experiences of accessing Canadian health care services: A grounded theory. International Journal for Equity in Health, 11, 55-55. doi: 10.1186/1475-9276$11-55$

Davies, A. A., Basten, A., \& Frattini, C. (2009). Migration: A social determinant of the health of migrants. Eurohealth, 16(1), 10-12.

de Haas, H. (2008). Migration and development: A theoretical perspective. Working paper 09. International Migration Institute. University of Oxford. Retrieved from http://www.imi.ox.ac.uk/publications/wp-09-08

de Haas, H. (2010a). The internal dynamics of migration processes: A theoretical inquiry. Journal of Ethnic and Migration Studies, 36(10), 1587-1617.

de Haas, H. (2010b). Migration and development: A theoretical perspective. International Migration Review, 44(1), 227-264.

de Haas, H. (2010c). Migration transitions: A theoretical and empirical inquiry into the developmental drivers of international migration. IMI Working Paper No 24. International Migration Institute, University of Oxford. Oxford. Retrieved from http://www.imi.ox.ac.uk/publications/wp-24-10

de Haas, H. (2011). The determinants of international migration (Vol. DEMIG Working Paper 2). International Migration Institute: University of Oxford de Haas, H. (2014a). Migration theory : Quo vadis? Arbeitspapier, Working Paper, Graue Literatur, Non-commercial literature. Oxford : International Migration Institute, University of Oxford. Retrieved from http://www.imi.ox.ac.uk/publications/working-papers/wp-100-2014 de Haas, H. (2014b). What drives human migration? Migration: A COMPAS Anthology. Retrieved from: http://compasanthology.co.uk/drives-human-migration/ de Luca, G., Ponzo, M., \& Andrés, A. R. (2013). Health care utilization by immigrants in Italy. International Journal of Health Care Finance and Economics, 13(1), 131. 
Deber, R. B. (2000). Getting what we pay for: Myths and realities about financing canada's health care system. Health Law in Canada, 21(2), 9-40.

Deber, R. B., Kraetschmer, N., Urowitz, S., \& Sharpe, N. (2005). Patient, consumer, client, or customer: What do people want to be called? Health Expectations, 8(4), 345-351. doi: 10.1111/j.1369-7625.2005.00352.x

del Pilar, J. A., \& Udasco, J. O. (2004). Deculturation: Its lack of validity. Cultural Diversity and Ethnic Minority Psychology, 10(2), 169-176. doi: http://dx.doi.org/10.1037/1099-9809.10.2.169

Denzin, N. K., \& Lincoln, Y. S. (2005). Paradigmatic controversies, contradictions, and emerging confluences. In N. K. Denzin \& Y. S. Lincoln (Eds.), The SAGE handbook of qualitative research (pp. 191-215). Thousand Oaks: SAGE Publications.

Derose, K. P., Bahney, B. W., Lurie, N., \& Escarce, J. J. (2009). Immigrants and health care access, quality, and cost. Medical Care Research and Review, 66(4), 355408.

Derose, K. P., Escarce, J. J., \& Lurie, N. (2007). Immigrants and health care: Sources of vulnerability. Health Affairs, 26(5), 1258-1268. doi: 10.1377/hlthaff.26.5.1258

DeWind, J., Kim, E. M., Skeldon, R., \& Yoon, I.-J. (2012). Korean development and migration. Journal of Ethnic and Migration Studies, 38(3), 371-388. doi: 10.1080/1369183X.2012.658543

Dey, I. (1999). Grounding grounded theory: Guidelines for qualitative inquiry. London: Academic Press.

Dias, S., Severo, M., \& Barros, H. (2008). Determinants of health care utilization by immigrants in Portugal. BMC Health Services Research, 8(1), 207.

DiCicco-Bloom, B., \& Crabtree, B. F. (2006). The qualitative research interview. Medical Education, 40(4), 314-321.

Dillon, D. R. (2012). Grounded theory and qualitative research The encyclopedia of applied linguistics: Blackwell Publishing Ltd.

Donabedian, A. (1988). The quality of care: How can it be assessed? Journal of American Medical Association, 260(12), 1743-1748. doi:

10.1001/jama.1988.03410120089033 
Dressler, W. W. (1993). Health in the African American community: Accounting for health inequalities. Medical Anthropology Quarterly, 7(4), 325-345.

Drew, C. J., Hardman, M. L., \& Hosp, J. L. (2008). Designing and conducting research in education. Thousand Oaks, London: SAGE Publications.

Eamranond, P. P., Legedza, A. T., Diez-Roux, A. V., Kandula, N. R., Palmas, W., Siscovick, D. S., \& Mukamal, K. J. (2009). Association between language and risk factor levels among Hispanic adults with hypertension, hypercholesterolemia, or diabetes. American Heart Journal, 157(1), 53-59. doi: 10.1016/j.ahj.2008.08.015

Eaton, S., Roberts, S., \& Turner, B. (2015). Delivering person centred care in long term conditions. BMJ, 350. doi: 10.1136/bmj.h181

Echevarria-Doan, S., \& Tubbs, C. (2005). Let's get grounded: Family therapy research and grounded theory. In D. H. Sprenkle \& F. P. Piercy (Eds.), Research methods in family therapy (2nd ed., pp. 41-62). New York, London: Guilford Press.

Eisenberg, L. (1977). Disease and illness distinctions between professional and popular ideas of sickness. Culture, Medicine and Psychiatry, 1(1), 9-23.

El-Wakil, A. (2011). Observations of the popularity and religious significance of bloodcupping (al-hijāāma) as an islamic medicine. Contemporary Islamic Studies, 2. Retrieved from: http://www.qscience.com/doi/pdfplus/10.5339/cis.2011.2

Ell, K., \& Castaneda, I. (1998). Health care seeking behavior. In S. Loue (Ed.), Handbook of immigrant health (pp. 125-143). New York: Springer.

Elliott, N., \& Higgins, A. (2012). Surviving grounded theory research method in an academic world: Proposal writing and theoretical frameworks. Grounded Theory Review, 11(2), 21-32.

Elliott, N., \& Lazenbatt, A. (2005). How to recognise a'quality'grounded theory research study. Australian Journal of Advanced Nursing, 22(3), 48-52.

Elliott, S. J., \& Gillie, J. (1998). Moving experiences: A qualitative analysis of health and migration. Health \& Place, 4(4), 327-339.

Emanuel, E. J. (1988). Do physicians have an obligation to treat patients with AIDS? New England Journal of Medicine, 318(25), 1686-1690.

Epstein, R. M., \& Street, R. L. (2011). The values and value of patient-centered care. Annals of Family Medicine, 9(2), 100-103. 
Epstein, S. (2006). Imagining the community: Newspapers, cyberspace and the [non-] construction of Korea-New Zealand identity. In H. Johnson \& B. Moloughney (Eds.), Asia in the making of New Zealand (pp. 147-162). Auckland: Auckland University Press.

Erlandsson, K., Warland, J., Cacciatore, J., \& Rådestad, I. (2013). Seeing and holding a stillborn baby: Mothers' feelings in relation to how their babies were presented to them after birth-findings from an online questionnaire. Midwifery, 29(3), 246-250. doi: http://dx.doi.org/10.1016/j.midw.2012.01.007

Faist, T. (2000). The volume and dynamics of international migration and transnational social spaces. Oxford, New York: Oxford University Press.

Faist, T. (2009). The crucial meso-level. In M. Martiniello \& J. Rath (Eds.), Selected studies in international migration and immigrant incorporation (pp. 59-90). Amsterdam: Amsterdam University Press.

Fassaert, T., Hesselink, A. E., \& Verhoeff, A. P. (2009). Acculturation and use of health care services by turkish and moroccan migrants: A cross-sectional populationbased study. BMC Public Health, 9, 332-332. doi: 10.1186/1471-2458-9-332

Fennelly, K. (2007). The" healthy migrant" effect. Minnesota Medicine, 90(3), 51-53.

Fiala, T. G. (2012). What do patients want? Technical quality versus functional quality: A literature review for plastic surgeons. Aesthetic Surgery Journal, 32(6), 751759.

Flatau, P. (2002). Hicks's the theory of wages: Its place in the history of neoclassical distribution theory. History of Economics Review 36, 44-65.

Flick, U. (2008). Designing qualitative research. London, Thousand Oaks, New Delhi: SAGE Publications Ltd.

Flint, N., \& Johnson, B. (2010). Towards fairer university assessment: Recognizing the concerns of students. London, New York: Taylor \& Francis.

Flores, G., \& Brotanek, J. (2005). The healthy immigrant effect: A greater understanding might help us improve the health of all children. Archives of Pediatrics \& Adolescent Medicine, 159(3), 295-297.

Foot, C., Naylor, C., \& Imison, C. (2010). The quality of GP diagnosis and referral. London: The King's Fund. 
Forrest, C. B., Glade, G. B., Baker, A. E., Bocian, A., von Schrader, S., \& Starfield, B. (2000). Coordination of specialty referrals and physician satisfaction with referral care. Archives of Pediatrics \& Adolescent Medicine, 154(5), 499.

Frederick, D. A., Forbes, G. B., \& Anna, B. (2008). Female body dissatisfaction and perceptions of the attractive female body in Ghana, the Ukraine, and the United States. Psihologijske Teme, 17(2), 203-219.

Galanis, P., Sourtzi, P., Bellali, T., Theodorou, M., Karamitri, I., Siskou, O., . . . Kaitelidou, D. (2013). Public health services knowledge and utilization among immigrants in Greece: A cross-sectional study. BMC Health Services Research, 13(1), 350 .

Gardner, A., Fedoruk, M., McCutcheon, H., Gardner, A., Fedoruk, M., \& McCutcheon, H. (2012). Discovering constructivist grounded theory's fit and relevance to researching contemporary mental health nursing practice. Australian Journal of Advanced Nursing, 30(2), 66-74.

Gasson, S. (2004). Rigor in grounded theory research: An interpretive perspective on generating theory from qualitative field studies. In W. Michael \& W. Amy (Eds.), The handbook of information systems research (pp. 79-102). Hershey, PA, USA: IGI Global.

Gauld, R., Blank, R., Burgers, J., Cohen, A. B., Dobrow, M., Ikegami, N., . . Wendt, C. (2012). The World Health Report 2008 - primary healthcare: How wide is the gap between its agenda and implementation in 12 high-income health systems? Healthcare Policy, 7(3), 38-58.

Gideon, J. (2011). Exploring migrants' health seeking strategies: The case of Latin American migrants in London. International Journal of Migration, Health and Social Care, 7(4), 197-208.

Gilgun, J. F. (2014). Chicago school traditions: Deductive qualitative analysis and grounded theory. San Bernardino, CA: CreateSpace Independent Publishing Platform.

Glaser, B. G. (1978). Theoretical sensitivity: Advances in the methodology of grounded theory. Mill Valley, Ca: Sociology Press.

Glaser, B. G. (1992). Emergence vs forcing: Basics of grounded theory analysis: Sociology Press. 
Glaser, B. G. (2001). The grounded theory perspective: Conceptualization contrasted with description. Mill Valley, CA.: Sociology Press.

Glaser, B. G. (2002). Conceptualization: On theory and theorizing using grounded theory. International Journal of Qualitative Methods, 1(2), 23-38.

Glaser, B. G. (2009). The novice GT researcher. Grounded Theory Review, 8(2), 1-21.

Glaser, B. G. (2010). The future of grounded theory. Grounded Theory Review, 9(2), 114. Retrieved from: http://groundedtheoryreview.com/2010/06/30/the-future-ofgrounded-theory1/

Glaser, B. G. (2011). Blocking conceptualization. Grounded Theory Review, 10(1), 115. Retrieved from: http://groundedtheoryreview.com/2011/04/11/blockingconceptualization1/

Glaser, B. G. (2012). Constructivist grounded theory? Grounded Theory Review, 11(1), 28-38. Retrieved from: http://groundedtheoryreview.com/2012/06/01/constructivist-grounded-theory/

Glaser, B. G. (2013). Staying open: The use of theoretical codes in GT. Grounded Theory Review, 12(1), 3-8. Retrieved from: http://groundedtheoryreview.com/2013/06/22/staying-open-the-use-oftheoretical-codes-in-gt/

Glaser, B. G., \& Holton, J. (2004). Remodeling grounded theory. Forum Qualitative Sozialforschung / Forum: Qualitative Social Research, 5(2), 47-68. Retrieved from: http://www.qualitative-research.net/index.php/fqs/article/view/607/1315

Glaser, B. G., \& Strauss, A. L. (1966). Awareness of dying. New Brunswik, London: Weidenfeld \& Nicolson.

Glaser, B. G., \& Strauss, A. L. (1967). The discovery of grounded theory: Strategies for qualitative research. Chicago: Aldine de Gruyter.

Godil, S. S., Parker, S. L., Zuckerman, S. L., Mendenhall, S. K., Devin, C. J., Asher, A. L., \& McGirt, M. J. (2013). Determining the quality and effectiveness of surgical spine care: Patient satisfaction is not a valid proxy. Spine Journal, 13(9), 1006-1012. doi: http://dx.doi.org/10.1016/j.spinee.2013.04.008

Goldsmith, R. L. (2012). Health care system structure and delivery in the Republic of Korea: Considerations for health care reform implementation in the United States. New Visions for Public Affairs, 4, 30-41. Retrieved from: 
https://nvpajournal.files.wordpress.com/2013/06/new-visions-for-public-affairsvolume-4.pdf

Gordon, M. (1964). Assimilation in American life: The role of race, religion and national origins. New York: Oxford University Press.

Greenwood, M. J., \& Hunt, G. L. (2003). The early history of migration research. International Regional Science Review, 26(1), 3-37.

Grigg, D. B. (1977). E. G. Ravenstein and the "Laws of migration". Journal of Historical Geography, 3(1), 41-54. doi: http://dx.doi.org/10.1016/03057488(77)90143-8

Grönroos, C. (1984). A service quality model and its marketing implications. European Journal of Marketing, 18(4), 36-44.

Grundy, J., \& Annear, P. (2010). Health-seeking behaviour studies: A literature review of study design and methods with a focus on Cambodia. Health Policy and Health Finance Knowledge Hub Working Paper Series no, 7.

Guillevin, L. (2012). Eosinophilic granulomatosis with polyangiitis (Churg-Strauss Syndrome). Presse médicale (Paris, France: 1983), 41(10), 1004.

Hagen-Zanker, J. (2008). Why do people migrate? A review of the theoretical literature. Maastrcht Graduate School of Governance Working Paper No. 2008/WP002. University Library of Munich. Gemany. Retrieved from http://ssrn.com/abstract=1105657 or http://dx.doi.org/10.2139/ssrn.1105657

Hall, K. D. (2004). The ethnography of imagined communities the cultural production of Sikh ethnicity in Britain. Annals of the American Academy of Political and Social Science, 595(1), 108-121.

Hallberg, L. R. (2006). The "core category" of grounded theory: Making constant comparisons. International Journal of Qualitative Studies on Health and Wellbeing, 1(3), 141-148.

Hammersley, M. (1987). Some notes on the terms 'validity'and 'reliability'[1]. British Educational Research Journal, 13(1), 73-82.

Han, I.-S., Hong, S.-O., Woo, J.-G., Byeon, K.-S., Hwang, S.-H., \& Lee, H.-C. (2007). A history of Koreans in New Zealand. Auckland, NZ: Korean History in New Zealand Compilation Committee. 
Harari, N., Davis, M., \& Heisler, M. (2008). Strangers in a strange land: Health care experiences for recent Latino immigrants in midwest communities. Journal of Health Care for the Poor and Underserved, 19(4), 1350-1367. doi: 10.1353/hpu.0.0086

Harmsen, J. A., Bernsen, R. M., Bruijnzeels, M. A., \& Meeuwesen, L. (2008). Patients' evaluation of quality of care in general practice: What are the cultural and linguistic barriers? Patient Educ Couns, 72(1), 155-162. doi: 10.1016/j.pec.2008.03.018

Harrington, C., \& Sprowl, B. (2012). Family members' experiences with viewing in the wake of sudden death. OMEGA - Journal of Death and Dying, 64(1), 65-82. doi: 10.2190/OM.64.1.e

Harris, J. R., \& Todaro, M. P. (1970). Migration, unemployment and development: A two-sector analysis. American Economic Review, 126-142.

Hasegawa, W., Yamauchi, Y., Yasunaga, H., Sunohara, M., Jo, T., Matsui, H., . . . Nagase, T. (2015). Factors that predict in-hospital mortality in eosinophilic granulomatosis with polyangiitis. Allergy, n/a-n/a. doi: 10.1111/all.12597

Hausmann-Muela, S., Muela Ribera, J., \& Nyamongo, I. (2003). Health-seeking behaviour and the health system response. In DCPP Working Paper No. 14. London School of Hygiene and Tropical Medicine. London. Retrieved from http://passinternational.org/site/images/stories/publications/DCPP_Working_Paper_14_No _Health_seeking_behaviour_and_the_health_system_response.pdf

Heath, H., \& Cowley, S. (2004). Developing a grounded theory approach: A comparison of Glaser and Strauss. Int J Nurs Stud, 41(2), 141-150.

Held, D. (1999). Global transformations: Politics, economics and culture. California: Stanford University Press.

Hellin, T. (2002). The physician-patient relationship: Recent developments and changes. Haemophilia, 8(3), 450-454.

Hesse-Biber, S. N., \& Leavy, P. (2010). The practice of qualitative research (2nd ed.). Los Angeles, London: SAGE Publications, Inc. 
Hibbard, J. H., \& Greene, J. (2013). What the evidence shows about patient activation: Better health outcomes and care experiences; fewer data on costs. Health Affairs, 32(2), 207-214.

Holton, J. A. (2008). Grounded theory as a general research methodology. Grounded Theory Review, 7(2), 67-93.

Holton, J. A. (2010). The coding process and its challenges. In A. Bryant \& K. Charmaz (Eds.), The Sage handbook of grounded theory (pp. 265-290). Los Angeles, London: SAGE Publications.

Holton, J. A., \& Walsh, I. (2016). Classic grounded theory: Applications with qualitative and quantitative data. Los Angeles, London: SAGE Publications.

Horton, S., \& Cole, S. (2011). Medical returns: Seeking health care in Mexico. Social Science \& Medicine, 72(11), 1846-1852.

Houghton, C. E., Casey, D., Shaw, D., \& Murphy, K. (2010). Ethical challenges in qualitative research: Examples from practice. Nurse Researcher, 18(1), 15-25. doi: $10.7748 / \mathrm{nr} 2010.10 .18 .1 .15 . c 8044$

Hu, H.-H., Kandampully, J., \& Juwaheer, T. D. (2009). Relationships and impacts of service quality, perceived value, customer satisfaction, and image: An empirical study. Service Industries Journal, 29(2), 111-125.

Hudson, B., Zarifeh, A., Young, L., \& Wells, J. E. (2012). Patients' expectations of screening and preventive treatments. Annals of Family Medicine, 10(6), 495502.

Hunt, L. M., Schneider, S., \& Comer, B. (2004). Should "acculturation" be a variable in health research? A critical review of research on US Hispanics. Social Science \& Medicine, 59(5), 973-986.

Immigration Bureau. (2005). Koreans overseas. Retrieved March 30, 2016, from http://www.immigration.go.kr/HP/IMM80/imm_04/imm_0407/imm_407010.jsp

Institute of Medicine. (2004). Patient safety: Achieving a new standard for care. Washington, DC: National Academies Press.

International Organization for Migration. (2013). International migration, health and human rights. Retrieved from http://www.ohchr.org/Documents/Issues/Migration/WHO_IOM_UNOHCHRPu blication.pdf 
Israel, M., \& Hay, I. (2006). Research ethics for social scientists. London, Thousand Oaks, New Delhi: SAGE Publications.

Jaber, L. A., Brown, M. B., Hammad, A., Zhu, Q., \& Herman, W. H. (2003). Lack of acculturation is a risk factor for diabetes in Arab immigrants in the U.S. Diabetes Care, 26(7), 2010-2014. doi: 10.2337/diacare.26.7.2010

Jackson, J. L., Chamberlin, J., \& Kroenke, K. (2001). Predictors of patient satisfaction. Social Science \& Medicine, 52(4), 609-620. doi: 10.1016/S02779536(00)00164-7

Jatrana, S., \& Crampton, P. (2009). Primary health care in New Zealand: Who has access? Health Policy, 93(1), 1-10. doi: http://dx.doi.org/10.1016/j.healthpol.2009.05.006

Jennings, D. (1986). The confusion between disease and illness in clinical medicine. Canadian Medical Association Journal, 135(8), 865.

Jennissen, R. (2007). Causality chains in the international migration systems approach. Population Research and Policy Review, 26(4), 411-436. doi: $10.2307 / 40230984$

Jeon, Y. H. (2004). The application of grounded theory and symbolic interactionism. Scandinavian Journal of Caring Sciences, 18(3), 249-256.

Jones, J. W. (2009). Selection of grounded theory as an appropriate research methodology for a dissertation: One student's perspective. Grounded Theory Review, 8(2), 23-34.

Jones, M., \& Alony, I. (2011). Guiding the use of grounded theory in doctoral studiesan example from the Australian film industry. International Journal of Doctoral Studies, 6, 95-114.

Jones, R. S. (2010). Health-care reform in Korea. OECD Economics Department Working Papers, 797. doi: http://dx.doi.org/10.1787/5kmbhk53x7nt-en

Jones, R. S. (2013). Education reform in Korea. OECD Economics Department Working Papers, No. 1067. Retrieved from: http://www.oecdilibrary.org/economics/education-reform-in-korea_5k43nxs1t9vh-en

Jumah, N. A., \& Duda, R. B. (2008). Comparison of the perception of ideal body images of Ghanaian men and women. African Journal of Health Sciences, 14(1), 54-60. 
Jun, M., Peterson, R. T., \& Zsidisin, G. A. (1998). The identification and measurement of quality dimensions in health care: Focus group interview results. Health Care Management Review, 23(4), 81-96.

Kaba, R., \& Sooriakumaran, P. (2007). The evolution of the doctor-patient relationship. International Journal of Surgery, 5(1), 57-65. doi: http://dx.doi.org/10.1016/j.ijsu.2006.01.005

Kahanec, M., \& Zimmermann, K. F. (2008). Migration and globalization: Challenges and perspectives for the research infrastructure. The Institute for the Study of Labor (IZA), (RatSWD Working Paper No. 51), 1-12. Retrieved from: http://ssrn.com/abstract=1445347 or http://dx.doi.org/10.2139/ssrn.1445347

Kennedy, S., McDonald, J. T., \& Biddle, N. (2006). The Healthy Immigrant Effect and immigrant selection: Evidence from four countries. SEDAP Research Paper No. 164. Ontario: McMaster University.

Kenny, M., \& Fourie, R. (2015). Contrasting classic, straussian, and constructivist grounded theory: Methodological and philosophical conflicts. The Qualitative Report, 20(8), 1270-1289.

Kiang, L., Yip, T., \& Fuligni, A. J. (2008). Multiple social identities and adjustment in young adults from ethnically diverse backgrounds. Journal of Research on Adolescence, 18(4), 643-670.

Kidney Health New Zealand. (2015). Kidney disease. from http://www.kidneys.co.nz/Kidney-Disease/Kidney-Disease/

Kim, A. E. (2009a). Demography, migration and multiculturalism in South Korea. The Asia-Pacific Journal, 7(6), 1-18.

Kim, B. S., \& Omizo, M. M. (2006). Behavioral acculturation and enculturation and psychological functioning among Asian American college students. Cultural Diversity and Ethnic Minority Psychology, 12(2), 245.

Kim, D. C., \& Yoon, H. K. (2003). Reality in paradise: A pilot study of Korean immigrants in New Zealand using the 1996 Census. In Y. Ishikawa \& A. Montanari (Eds.), The new geography of human mobility: Inequality trends? (pp. 85-102). Rome: Società geografica italiana.

Kim, D. S. (2010). Introduction: Health of the health care system in Korea. Social Work in Public Health, 25(2), 127-141. 
Kim, H.-J., \& Ruger, J. P. (2008). Pharmaceutical reform in South Korea and the lessons it provides. Health Affairs, 27(4), w260-w269. doi: 10.1377/hlthaff.27.4.w260

Kim, J., \& Chan, M. M. (2004). Acculturation and dietary habits of Korean Americans. British Journal of Nutrition, 91(03), 469-478.

Kim, M., Han, H.-R., Kim, K. B., \& Duong, D. N. (2002). The use of traditional and western medicine among Korean American elderly. Journal of Community Health, 27(2), 109-120.

Kim, M. H., Kim, C. Y., Park, Y. K., Park, Y. A., \& Cho, H. J. (2015). 건강검진은 어떻게 “산업”이 되었나? [How medical check-ups became an industry?]. In Y. K. Park (Ed.), PHI Research Report 2015-02. Seoul: People's Health Institute.

Kim, M. S. (2015). The subjective well-being of Korean children and its policy implications. Seoul: Ministry of Health and Welfare.

Kim, S., \& Rew, L. (1994). Ethnic identity, role integration, quality of life, and depression in Korean-American women. Archives of Psychiatric Nursing, 8(6), 348-356. doi: http://dx.doi.org/10.1016/0883-9417(94)90003-5

Kim, Y. Y. (2006). From ethnic to interethnic the case for identity adaptation and transformation. Journal of Language and Social Psychology, 25(3), 283-300.

Kim, Y. Y. (2008). Intercultural personhood: Globalization and a way of being. International Journal of Intercultural Relations, 32(4), 359-368. doi: http://dx.doi.org/10.1016/j.ijintrel.2008.04.005

Kim, Y. Y. (2009b). The identity factor in intercultural competence. In D. K. Deardorff (Ed.), The Sage handbook of intercultural competence (pp. 53-65). California: SAGE Publications Ltd.

King, R. (2012). Theories and typologies of migration: An overview and a primer. Willy Brandt Series of Working Papers in International Migration and Ethnic Relations 3/12. Malmö Institute for Studies of Migration, Diversity and Welfare (MIM) Malmö University. Sweden.

Koo, B. G. (2004). Adaptation process and the mental health of Korean immigrants. In S. Tse, A. Thapliyal, S. Garg, G. Lim, \& M. Chatterji (Eds.), Inaugural international Asian health conference: Asian health and wellbeing, now and into 
the future (pp. 79-90). New Zealand: The University of Auckland, School of Population Health.

Kools, S. (1999). Self-protection in adolescents in foster care. Journal of Child and Adolescent Psychiatric Nursing, 12(4), 139-152.

Kools, S., McCarthy, M., Durham, R., \& Robrecht, L. (1996). Dimensional analysis: Broadening the conception of grounded theory. Qualitative Health Research, 6(3), 312-330. doi: 10.1177/104973239600600302

Kraetschmer, N., Sharpe, N., Urowitz, S., \& Deber, R. B. (2004). How does trust affect patient preferences for participation in decision-making? Health Expectations, 7(4), 317-326. doi: 10.1111/j.1369-7625.2004.00296.x

Krugman, P. (2011, April 20). Patients are not consumers. The New York Times. Retrieved from http://www.nytimes.com/2011/04/22/opinion/22krugman.html?_r=0

Ku, L., \& Matani, S. (2001). Left out: Immigrants' access to health care and insurance. Health Affairs, 20(1), 247-256. doi: 10.1377/hlthaff.20.1.247

Kuehn, B. M. (2009). States explore shared decision making. Journal of the American Medical Association, 301(24), 2539-2541.

Kulis, S. S., Marsiglia, F. F., Kopak, A. M., Olmsted, M. E., \& Crossman, A. (2012). Ethnic identity and substance use among Mexican-heritage preadolescents: Moderator effects of gender and time in the United States. Journal of Early Adolescence, 32(2), 165-199. doi: 10.1177/0272431610384484

Kumpikaite, V., \& Zickute, I. (2012). Synergy of migration theories: Theoretical insights. Engineering Economics, 23(4), 387-394.

Kunst, J. R., \& Sam, D. L. (2013). Expanding the margins of identity: A critique of marginalization in a globalized world. International Perspectives in Psychology: Research, Practice, Consultation, 2(4), 225.

Kurekova, L. (2011). Theories of migration: Conceptual review and empirical testing in the context of the EU East-West flows Interdisciplinary conference on Migration. Economic Change, Social Challenge. UK: University College London.

Kwok, K., McCallin, A., \& Dickson, G. (2012). Working through preconception: Moving from forcing to emergence. Grounded Theory Review, 11(2), 33-43. 
Kwon, S.-H. (2013). End-of-life care in Korea: Issues and trends. Japanese Journal of Nursing and Health Sciences, 11, 54-56.

Kwon, S., Lee, T. J., \& Kim, C. Y. (2015). Health systems in transition: Republic of Korea health system review. Manila : WHO Regional Office for the Western Pacific: Retrieved from http://iris.wpro.who.int/handle/10665.1/11358.

Kwon, T.-H. (1997). International migration of Koreans and the Korean community in China. Korea Journal of Population and Development, 26(1), 1-18.

Lakey, P. N. (2003). Acculturation: A review of the literature. Intercultural Communication Studies, 12(2), 103-118.

Lang, H. G. (1987). Health policy formulation in New Zealand. The International Journal of Health Planning and Management, 2(5), 143-160. doi: 10.1002/hpm.4740020516

Lara, M., Gamboa, C., Kahramanian, M. I., Morales, L. S., \& Hayes Bautista, D. E. (2005). Acculturation and Latino health in the United States: A review of the literature and its sociopolitical context. Annu. Rev. Public Health, 26, 367-397.

Laroche, M., Kim, C., Tomiuk, M. A., \& Bélisle, D. (2005). Similarities in italian and greek multidimensional ethnic identity: Some implications for food consumption. Canadian Journal of Administrative Sciences / Revue Canadienne des Sciences de l'Administration, 22(2), 143-167. doi: 10.1111/j.19364490.2005.tb00715.x

Leclere, F., Jensen, L., \& Biddlecom, A. (1994). Health care utilization, family context, and adaptation among immigrants to the United States. Journal of Health and Social Behavior, 35, 370-384.

Leduc, N., \& Proulx, M. (2004). Patterns of health services utilization by recent immigrants. Journal of Immigrant Health, 6(1), 15-27.

Lee, E. S. (1966). A theory of migration. Demography, 3(1), 47-57.

Lee, J.-H., Choi, Y.-J., Lee, S. H., Sung, N.-J., Kim, S.-Y., \& Hong, J. Y. (2013). Association of the length of doctor-patient relationship with primary care quality in seven family practices in Korea. Journal of Korean Medical Science, 28(4), 508-515. doi: 10.3346/jkms.2013.28.4.508 
Lee, J., Carling, J., \& Orrenius, P. (2014). The international migration review at 50: Reflecting on half a century of international migration research and looking ahead. International Migration Review, 48, S3-S36. doi: 10.1111/imre.12144

Lee, J., Park, S., Choi, K., \& Kwon, S.-M. (2010). The association between the supply of primary care physicians and population health outcomes in Korea. Family Medicine, 42(9), 628-635.

Lee, J., Wachholtz, A., \& Choi, K.-H. (2014). A review of the Korean cultural syndrome hwa-byung: Suggestions for theory and intervention. Asia Taepyongyang Sangdam Yongu, 4(1), 49.

Lee, J. H. (2014). A cross-cultural study of hwa-byung with middle-aged women between native Koreans in South Korea and Korean immigrants in the United States. (PhD), University of Iowa. Retrieved from http://ir.uiowa.edu/cgi/viewcontent.cgi?article=5393\&context=etd

Lee, J. H. (2015). Hwabyung and depressive symptoms among Korean immigrants. Social Work in Mental Health, 13(2), 159-185. doi: $10.1080 / 15332985.2013 .812538$

Lee, J. Y., Kearns, R. A., \& Friesen, W. (2010). Seeking affective health care: Korean immigrants' use of homeland medical services. Health \& Place, 16(1), 108-115. doi: http://dx.doi.org/10.1016/j.healthplace.2009.09.003

Lee, N. C. (2012). A study on the structure of quality of life in OECD member countries. Health and Social Welfare Review, 32(2), 5-40.

Lee, S., Chen, L., He, X., Miller, M. J., \& Juon, H.-S. (2013). A cluster analytic examination of acculturation and health status among Asian Americans in the Washington DC metropolitan area, United States. Social Science \& Medicine, 96, 17-23. doi: http://dx.doi.org/10.1016/j.socscimed.2013.07.007

Lee, W.-C., \& Lee, S.-Y. (2010). National health screening program of Korea. J Korean Med Assoc, 53(5), 363-370.

Lee, W.-Y., \& Shaw, I. (2014). The impact of out-of-pocket payments on health care inequity: The case of national health insurance in South Korea. International Journal of Environmental Research and Public Health, 11(7), 7304-7318. doi: 10.3390/ijerph110707304 
Lempert, L. B. (2010). Asking questions of the data: Memo writing in the grounded theory tradition. In A. Bryant \& K. Charmaz (Eds.), The SAGE handbook of grounded theory: Paperback edition (pp. 245-265). Los Angeles, London: SAGE Publications.

Lenz, K., Bauer-Dubau, K., \& Jelinek, T. (2006). Delivery of medical care for migrants in Germany: Delay of diagnosis and treatment. Journal of Travel Medicine, 13(3), 133-137. doi: 10.1111/j.1708-8305.2006.00030.x

Leplege, A., Gzil, F., Cammelli, M., Lefeve, C., Pachoud, B., \& Ville, I. (2007). Person-centredness: Conceptual and historical perspectives. Disability and Rehabilitation, 29(20-21), 1555-1565.

Levinson, W., Kao, A., Kuby, A., \& Thisted, R. (2005). Not all patients want to participate in decision making. Journal of General Internal Medicine, 20(6), 531-535. doi: 10.1111/j.1525-1497.2005.04101.x

Lidguard, J., \& Yoon, H.-K. (1998). The employment experiences of recent Korean immigrants in New Zealand. Labour, Employment and Work in New Zealand.

Lien, E., Nafstad, P., \& Rosvold, E. O. (2008). Non-western immigrants' satisfaction with the general practitioners' services in Oslo, Norway. International Journal for Equity in Health, 7(1), 7-7. doi: 10.1186/1475-9276-7-7

Lingard, L., Albert, M., \& Levinson, W. (2008). Grounded theory, mixed methods, and action research. BMJ, 337. doi: 10.1136/bmj.39602.690162.47

Lipson, J. G., Weinstein, H. M., Gladstone, E. A., \& Sarnoff, R. H. (2003). Bosnian and Soviet refugees' experiences with health care. Western Journal of Nursing Research, 25(7), 854-871.

Lopez, I. (2008). "But you don't look Puerto Rican": The moderating effect of ethnic identity on the relation between skin color and self-esteem among Puerto Rican women. Cultural Diversity and Ethnic Minority Psychology, 14(2), 102-108. doi: 10.1037/1099-9809.14.2.102

Love, A. S., Yin, Z., Codina, E., \& Zapata, J. T. (2006). Ethnic identity and risky health behaviors in school-age Mexican-American children 1, 2. Psychological Reports, 98(3), 735-744.

MacKian, S. (2003). A review of health seeking behaviour: Problems and prospects. Health Systems Development Programme. University of Manchester. Retrieved 
from http://r4d.dfid.gov.uk/pdf/outputs/healthsysdev_kp/05-

03_health_seeking_behaviour.pdf

Markey, K., Tilki, M., \& Taylor, G. (2014). Reflecting on the challenges of choosing and using a grounded theory approach. Nurse Researcher, 22(2), 16-22.

Martimianakis, M. A., Maniate, J. M., \& Hodges, B. D. (2009). Sociological interpretations of professionalism. Medical Education, 43(9), 829-837.

Martin, P. (2013). The global challenge of managing migration. Population Bulletin, $68(2)$.

Maruthappu, M., Ologunde, R., \& Gunarajasingam, A. (2013). Is health care a right? Health reforms in the USA and their impact upon the concept of care. Annals of Medicine and Surgery, 2(1), 15-17. doi: http://dx.doi.org/10.1016/S20490801(13)70021-9

Massey, D. S. (1988). Economic development and international migration in comparative perspective. Population and Development Review, 14(3), 383-413. doi: http://onlinelibrary.wiley.com/journal/10.1111/\%28ISSN\%2917284457/issues

Massey, D. S. (1999). Why does immigration occur? A theoretical synthesis. In C. Hirschman, P. Kasinitz, \& J. DeWind (Eds.), The handbook of international migration: The American experience. New York: Russell Sage Foundation.

Massey, D. S. (2003). Patterns and processes of international migration in the 21 st century. Paper presented at the Conference on African Migration in Comparative Perspective, Johannesburg, South Africa, June 4-7.

Massey, D. S., Arango, J., Hugo, G., Kouaouci, A., Pellegrino, A., \& Taylor, J. E. (1993). Theories of international migration: A review and appraisal. Population and Development Review, 19(3), 431-466. doi: 10.2307/2938462

Matsudaira, T. (2006). Measures of psychological acculturation: A review. Transcultural Psychiatry, 43(3), 462-487. doi: 10.1177/1363461506066989

Matsunaga, M., Hecht, M. L., Elek, E., \& Ndiaye, K. (2010). Ethnic identity development and acculturation: A longitudinal analysis of Mexican-heritage youth in the Southwest United States. Journal of Cross-Cultural Psychology, 41(3), 410-427. doi: 10.1177/0022022109359689 
Mavetera, N., \& Kroeze, J. H. (2009). Practical considerations in grounded theory research. Sprouts: Working Papers on Information Systems, 9(32). Retrieved from: http://sprouts.aisnet.org/9-32

McCormack, B., Karlsson, B., Dewing, J., \& Lerdal, A. (2010). Exploring personcentredness: A qualitative meta-synthesis of four studies. Scandinavian Journal of Caring Sciences, 24(3), 620-634. doi: 10.1111/j.1471-6712.2010.00814.x

McDonald, J. T., \& Kennedy, S. (2004). Insights into the 'healthy immigrant effect': Health status and health service use of immigrants to Canada. Social Science \& Medicine, 59(8), 1613-1627. doi: 10.1016/j.socscimed.2004.02.004

McLaughlin, H. (2009). What's in a name: 'Client', 'patient', 'customer', 'consumer', 'expert by experience', 'service user'—what's next? British Journal of Social Work, 39(6), 1101-1117. doi: 10.1093/bjsw/bcm155

Mcllwaine, C., Cock, J. C., \& Linneker, B. (2011). No longer invisible: The Latin American community in London. Trust for London. London. Retrieved from http://www.geog.qmul.ac.uk/docs/research/latinamerican/48637.pdf

Mead, N., \& Roland, M. (2009). Understanding why some ethnic minority patients evaluate medical care more negatively than white patients: A cross sectional analysis of a routine patient survey in english general practices. $B M J$, $339(7722)$.

Medical Council of New Zealand. Public and private health systems. Retrieved Feb 24, 2016, from https://www.mcnz.org.nz/alpinfo/public-and-private-health-systems Medical screening. (2014). Visit Medical Korea. Retrieved April 7, 2016, from http://english.visitmedicalkorea.com/english/pt/packages/BD_detail02.do?packa geSeq=276\&packageMenuDivCd=1001\&packageCateCd=1002\&menuGB=1

Mehta, S. (2012). Health needs assessment of Asian people living in the Auckland region. Auckland: Northern DHB Support Agency.

Merriam, S. B. (1997). Qualitative research and case study applications in education: Revised and expanded from case study research in education. San Francisco: Jossey-Bass Publishers.

Mertens, D. M. (1998). Research methods in education and psychology: Integrating diversity with quantitative \& qualitative approaches. Thousand Oaks, London: Sage Publications. 
Migge, B., \& Gilmartin, M. (2011). Migrants and healthcare: Investigating patient mobility among migrants in Ireland. Health and Place, 17(5), 1144-1149. doi: 10.1016/j.healthplace.2011.05.002

Migrant crisis: Migration to Europe explained in graphics. (2015, December 22). BBC News. Retrieved from http://www.bbc.com/news/world-europe-34131911

Miller, M. J., \& Lim, R. H. (2011). The importance of acculturation and cultural values in counseling Asian American men. In W. M. Liu, D. K. Iwamoto, \& M. H. Chae (Eds.), Culturally responsive counseling with Asian American men (pp. 3962). New York: Taylor \& Francis.

Mills, J., Bonner, A., \& Francis, K. (2006). The development of constructivist grounded theory. International Journal of Qualitative Methods, 5(1), 25-35.

Mills, J., Chapman, Y., Bonner, A., \& Francis, K. (2007). Grounded theory: A methodological spiral from positivism to postmodernism. Journal of Advanced Nursing, 58(1), 72-79. doi: 10.1111/j.1365-2648.2007.04228.x

Ministry of Foreign Affairs. (2015). 2015 재외동포현황 [2015 Overseas Koreans].

Retrieved from http://www.mofa.go.kr/travel/overseascitizen/.

Ministry of Health. (2006). Asian health chart book 2006. Wellington: Author.

Ministry of Health. (2010). The New Zealand health survey: Objectives and topic areas august 2010. Wellington: Author.

Ministry of Health. (2012a). Health expenditure trends in New Zealand 2000-2010.

Wellington: Author.

Ministry of Health. (2012b). New Zealand national health survey 2011/12. Wellington: Author.

Ministry of Health. (2014a). Annual update of key results 2013/14: New Zealand health survey. Wellington: Author.

Ministry of Health. (2014b). Annual update of key results 2013/14: New Zealand health survey. Wellington: Author.

Ministry of Health. (2015a). Accident cover. Retrieved Feb 24, 2016, from http://www.health.govt.nz

Ministry of Health. (2015b). District health boards. Retrieved Feb 225, 2016, from http://www.health.govt.nz 
Ministry of Health. (2016). Enrolment in a primary health organisation. Retrieved Feb 25, 2016, from http://www.health.govt.nz/

Ministry of Health and Welfare. (2011). 2011 년 상반기 건강보험 주요 통계 [The major health insurance statistics for the first half of 2011]. Retrieved from: http://www.mohw.go.kr/front_new/jb/sjb030301vw.jsp

Ministry of Health and Welfare. (2012). 2012 의료기관 개설 및 의료법인 설립 운영 편람 [2012 Manual for medical institutions establishment and medical corporation operation]. Korea: Retrieved from http://www.mohw.go.kr/front_new/gm/sgm0601vw.jsp?PAR_MENU_ID=13\& MENU_ID=1304020304\&page=1\&CONT_SEQ=293244.

Ministry of the Interior. (2015). 2015 년도 지방자치단체 외국인 주민 현황 [2015 Foreign residents in each province]. Seoul: Author. Retrieved from http://www.moi.go.kr/frt/bbs/type001.

Minkoff, H., \& Ecker, J. (2015). Physicians' obligations to patients infected with ebola: Echoes of acquired immune deficiency syndrome. American Journal of Obstetrics and Gynecology, 212(4), 456.e451-456.e454. doi: http://dx.doi.org/10.1016/j.ajog.2014.12.026

Mishra, S., Kusuma, Y. S., \& Babu, B. V. (2013). Concepts of health and illness: Continuity and change among migrant tribal community in an Eastern Indian city. Anthropological Notebooks, 19(3), 61-69.

Mitchell, P. H. (2008). Defining patient safety and quality care. In R. G. Hughes (Ed.), Patient safety and quality: An evidence-based handbook for nurses; Chapter 1 (pp. 1-5). Rockville (MD): Agency for Healthcare Research and Quality (US).

Mohammad, A. A. S., \& Alhamadani, S. Y. M. (2011). Service quality perspectives and customer satisfaction in commercial banks working in Jordan. Euro Journals Publishing, 14, 60-72.

Mold, A. (2015). Making British patients into consumers. The Lancet, 385(9975), 12861287. doi: 10.1016/S0140-6736(15)60672-9

Moore, A. M., \& Barker, G. G. (2012). Confused or multicultural: Third culture individuals' cultural identity. International Journal of Intercultural Relations, 36(4), 553-562. doi: http://dx.doi.org/10.1016/j.ijintrel.2011.11.002 
Morawska, E. (2007). International migration: Its various mechanisms and different theories that try to explain it. Willy Brandt Series of Working Papers in International Migration and Ethnic Relations. Malmo University. Sweden.

Morgan, D. L. (2008). Snowball sampling. In L. M. Given (Ed.), The Sage encyclopedia of qualitative research methods (pp. 816-817). Thousand Oaks, CA: SAGE Publications.

Morgan, S., \& Yoder, L. H. (2012). A concept analysis of person-centered care. Journal of Holistic Nursing, 30(1), 6-15.

Morris, C., Vokes, R., \& Chang, S. (2007). Social exclusion and church in the experiences of Korean migrant families in Christchurch. Sites: New Series, 4(2), $11-31$

Morse, J. M. (2009). Tussles, tensions, and resolutions. In J. M. Morse, P. N. Stern, J. Corbin, B. Bowers, K. Charmaz, \& A. E. Clarke (Eds.), Developing grounded theory: The second generation (pp. 13-23). Walnut Creek, CA: Left Coast Press.

Mosadeghrad, A. M. (2013). Healthcare service quality: Towards a broad definition. International Journal of Health Care Quality Assurance, 26(3), 203-219.

Mossma, D. (2014). Are the people we serve 'patients' or 'customers'? Current Psychiatry, 13(3), 40-43.

Nagel, J. (1994). Constructing ethnicity: Creating and recreating ethnic identity and culture. Social Problem New York-, 41, 152-152.

Naidu, A. (2009). Factors affecting patient satisfaction and healthcare quality. International Journal of Health Care Quality Assurance, 22(4), 366-381.

National Health Insurance Service. (2014). National health insurance system of Korea 2014: Universal health coverage for the world. Retrieved from http://www.coopami.org/en/countries/countries/south_korea/social_protection/p df/social_protection05.pdf.

Neuwelt, P., \& Matheson, D. (2012). New Zealand's journey towards people-centred care. International Journal of Person Centered Medicine, 2(1), 7. doi: 10.5750/ijpcm.v2i1.178

New Zealand Immigration Service. (2004). Migrants' experiences of New Zealandpilot survey report. Wellington: Author. Retrieved from www.immigration.govt.nz. 
Ng, K., \& Hase, S. (2008). Grounded suggestions for doing a grounded theory business research. Electronic Journal of Business Research Methods, 6(2), 155-170.

Ninnemann, K. (2012). Hwa-byung. In S. Loue \& M. Sajatovic (Eds.), Encyclopedia of immigrant health (pp. 862-863). New York: Springer.

Noels, K. A., Pon, G., \& Clément, R. (1996). Language, identity, and adjustment the role of linguistic self-confidence in the acculturation process. Journal of Language and Social Psychology, 15(3), 246-264.

Noh, M., Lee, Y., Yun, S.-C., Lee, S.-I., Lee, M.-S., \& Khang, Y.-H. (2006). Determinants of hospital closure in South Korea: Use of a hierarchical generalized linear model. Social Science \& Medicine, 63(9), 2320-2329. doi: http://dx.doi.org/10.1016/j.socscimed.2006.05.026

Noh, S., \& Kaspar, V. (2003). Perceived discrimination and depression: Moderating effects of coping, acculturation, and ethnic support. American Journal of Public Health, 93(2), 232-238.

Norris, P., Ng, L. F., Kershaw, V., Hanna, F., Wong, A., Talekar, M., . . Cheong, L. (2010). Knowledge and reported use of antibiotics amongst immigrant ethnic groups in New Zealand. Journal of Immigrant and Minority Health, 12(1), 107112.

O'Brien, M. J., Alos, V. A., Davey, A., Bueno, A., \& Whitaker, R. C. (2014). Acculturation and the prevalence of diabetes in US Latino adults, national health and nutrition examination survey 2007-2010. Preventing Chronic Disease, 11, E176. doi: 10.5888/pcd11.140142

O'Donnell, C., Higgins, M., Chauhan, R., \& Mullen, K. (2007). "They think we're ok and we know we're not". A qualitative study of asylum seekers' access, knowledge and views to health care in the UK. BMC Health Services Research, $7(1), 75$.

O'Donnell, C. A., Higgins, M., Chauhan, R., \& Mullen, K. (2008). Asylum seekers' expectations of and trust in general practice: A qualitative study. British Journal of General Practice, 58(557), e1-e11. doi: 10.3399/bjgp08X376104

O'Reilly, K., \& Benson, M. (2009). Lifestyle migration: Escaping to the good life? In M. Benson \& K. O'Reilly (Eds.), Lifestyle migration: Expectation, aspirations and experiences (pp. 1-13). London: Ashgate. 
Ock, M., Kim, J.-E., Jo, M.-W., Lee, H.-J., Kim, H. J., \& Lee, J. Y. (2014). Perceptions of primary care in Korea: A comparison of patient and physician focus group discussions. BMC Family Practice, 15(1), 178-178. doi: 10.1186/s12875-0140178-5

OECD. (2002). Review of the Korean health care system. Paris: OECD Retrieved from http://www.mohw.go.kr/databank/koreahealth.pdf.

OECD. (2006). Where immigrant students succeed: A comparative review of performance and engagement in Pisa 2003. Paris: PISA, OECD Publishing.

OECD. (2011). OECD launches Your Better Life Index. Retrieved from http://www.oecd.org/economy/oecdlaunchesyourbetterlifeindex.htm.

OECD. (2012). OECD reviews of health care quality: Korea: Raising standards. Paris: OECD Publishing. Retrieved from http://dx.doi.org/10.1787/9789264173446-en.

OECD. (2013). Health at a glance 2013: OECD indicators: OECD Publishing. http://dx.doi.org/10.1787/health_glance-2013-en.

OECD/European Union. (2015). Indicators of immigrant integration 2015: OECD Publishing.

Oh, D.-Y., Kim, J.-E., Lee, C.-H., Lim, J.-S., Jung, K.-H., Seog Heo, D., . . Kyeong Kim, N. (2004). Discrepancies among patients, family members, and physicians in Korea in terms of values regarding the withholding of treatment from patients with terminal malignancies. Cancer, 100(9), 1961-1966. doi: 10.1002/cncr.20184

Oh, J. E., Kang, D. K., Shin, J. J., Lee, S. L., Lee, S. B., \& Chung, K. (2011). Migration profile of the Republic of Korea. IOM MRTC Research Report Series, No. 2011-01. IOM Migration Research and Training Centre. Republic of Korea. Retrieved from http://publications.iom.int/system/files/pdf/mp_korea.pdf Omonzejele, P. F. (2005). Obligation of non-maleficence: Moral dilemma in physicianpatient relationship. Journal of Medicine and Biomedical Research, 4(1), 22-30.

Orb, A., Eisenhauer, L., \& Wynaden, D. (2001). Ethics in qualitative research. Journal of Nursing Scholarship, 33(1), 93-96.

Organista, P. B., Marin, G., \& Chun, K. M. (2010). The psychology of ethnic groups in the United States. Thousand Oaks, California: SAGE Publications, Inc. 
Ormond, M. (2014). Harnessing "diasporic" medical mobilities. In F. Thomas \& J. Gideon (Eds.), Migration, health and inequality (pp. 150-162). London: Zed Books.

Osborn, R., \& Moulds, D. (2014). The Commonwealth Fund 2014 international health policy survey of older adults in eleven countries. Retrieved from: http://www.commonwealthfund.org/ /media/files/publications/in-theliterature/2014/nov/pdf_1787_commonwealth_fund_2014_intl_survey_chartpac k.pdf

Oza-Frank, R., \& Cunningham, S. A. (2010). The weight of US residence among immigrants: A systematic review. Obesity Reviews, 11(4), 271-280. doi: 10.1111/j.1467-789X.2009.00610.x

Ozer, S. (2013). Theories and methodologies in acculturation psychology: The emergence of a scientific revolution? Psychological Studies, 58(3), 339-348. doi: 10.1007/s12646-013-0203-0

Padilla, A. M. (2006). Bicultural social development. Hispanic Journal of Behavioral Sciences, 28(4), 467-497. doi: 10.1177/0739986306294255

Padilla, Y. C., \& Villalobos, G. (2007). Cultural responses to health among Mexican American women and their families. Family \& Community Health, 30, S24-S33.

Palliative Care Council. (2013). Deaths in New Zealand: Regional and Ethnic Projections, 2014-2026. Working Paper No. 3, April 2014. Wellington: Cancer Control New Zealand.

Palmer, P. J. (2007). A new professional: The aims of education revisited. Change: The Magazine of Higher Learning, 39(6), 6-13.

Papanikolaou, V., \& Zygiaris, S. (2014). Service quality perceptions in primary health care centres in Greece. Health Expectations, 17(2), 197-207. doi:

10.1111/j.1369-7625.2011.00747.x

Parasuraman, A., Zeithaml, V. A., \& Berry, L. L. (1985). A conceptual model of service quality and its implications for future research. Journal of Marketing, 49(4), 4150. doi: $10.2307 / 1251430$

Park, C. H., Shin, D. W., Choi, J. Y., Kang, J., Baek, Y. J., Mo, H. N., . . Park, S. (2012). Determinants of the burden and positivity of family caregivers of terminally ill cancer patients in Korea. Psycho-Oncology, 21(3), 282-290. 
Park, H.-J. (2014). Living with 'Hwa-byung': The psycho-social impact of elder mistreatment on the health and well-being of older people. Aging \& Mental Health, 18(1), 125-128. doi: 10.1080/13607863.2013.814103

Park, J., Myers, D., Kao, D., \& Min, S. (2009). Immigrant obesity and unhealthy assimilation: Alternative estimates of convergence or divergence, 1995-2005. Social Science \& Medicine, 69(11), 1625-1633. doi: http://dx.doi.org/10.1016/j.socscimed.2009.09.008

Park, J. M. (2014, Nov 04). South Korean children come last in 30-nation happiness survey. Daily Mail. Retrieved from http://www.dailymail.co.uk/wires/reuters/article-2820017/South-Koreanchildren-finish-happiness-survey.html

Park, S., Soumerai, S. B., Adams, A. S., Finkelstein, J. A., Jang, S., \& Ross-Degnan, D. (2005). Antibiotic use following a Korean national policy to prohibit medication dispensing by physicians. Health Policy and Planning, 20(5), 302-309. doi: 10.1093/heapol/czi033

Park, Y.-B. (2004). South Korea: Balancing labor demand with strict controls. Retrieved from Migration Policy Institute website: http://www.migrationpolicy.org/article/south-korea-balancing-labor-demandstrict-controls/

Paterson, K. (2015). Health and well-being: Health professionals. Grown Ups. Retrieved 15 March, 2015, from http://www.kiwifamilies.co.nz/articles/familydoctor-gp/

Patton, M. Q. (2001). Qualitative research \& evaluation methods (3rd ed.). Thousand Oaks, London: SAGE Publications, Inc.

Peck, B. M., Asch, D. A., Goold, S. D., Roter, D. L., Ubel, P. A., McIntyre, L. M., . . Tulsky, J. A. (2001). Measuring patient expectations: Does the instrument affect satisfaction or expectations? Medical Care, 39(1), 100-108.

Pellegrino, E. D. (2002). Professionalism, profession and the virtues of the good physician. Mount Sinai Journal of Medicine, 69(6), 378.

Pelzang, R. (2010). Time to learn: Understanding patient-centred care. British Journal of Nursing, 19(14), 912. 
Pender, N. J., Murdaugh, C. L., \& Parsons, M. A. (2002). Health promotion in nursing practice (4th Ed.). Upper Saddle River, NJ: Prentice Hall.

Pérez-Escamilla, R., \& Putnik, P. (2007). The role of acculturation in nutrition, lifestyle, and incidence of type 2 diabetes among Latinos. The Journal of Nutrition, 137(4), 860-870.

Perez, A. (2015). Acculturation, health literacy, and illness perceptions of hypertension among Hispanic adults. Journal of Transcultural Nursing, 26(4), 386-394. doi: $10.1177 / 1043659614524785$

Pérez, D. J., Fortuna, L., \& Alegria, M. (2008). Prevalence and correlates of everyday discrimination among US Latinos. Journal of Community Psychology, 36(4), 421.

Pergert, P. (2009). Methodological learning-by-doing: Challenges, lessons learned and rewards. Grounded Theory Review, 8(2), 65-75.

Petersen, M. B. (1988). Measuring patient satisfaction: Collecting useful data. Journal of Nursing Care Quality, 2(3), 25-35.

Petrie, K. J., \& Weinman, J. (2012). Patients' perceptions of their illness: The dynamo of volition in health care. Current Directions in Psychological Science, 21(1), 60-65. doi: 10.1177/0963721411429456

Phinney, J. S. (1990). Ethnic identity in adolescents and adults: Review of research. Psychological Bulletin, 108(3), 499.

Phinney, J. S., \& Ong, A. D. (2007). Conceptualization and measurement of ethnic identity: Current status and future directions. Journal of Counseling Psychology, 54(3), 271.

Piore, M. J. (1979). Birds of passage: Migrant labor and industrial societies. Cambridge, New York: Cambridge University Press.

Piterman, L., \& Koritsas, S. (2005). Part ii. General practitioner-specialist referral process. Internal Medicine Journal, 35(8), 491-496. doi: 10.1111/j.14455994.2005.00860.x

Pleizier, T. (2010). Religious involvement in hearing sermons: A grounded theory study in empirical theology and homiletics. Delf, The Netherlands: Eburon Academic Publishers. 
Polit, D. F., \& Beck, C. T. (2013). Essentials of nursing research: Appraising evidence for nursing practice (8th ed.). Philadelphia: Wolters Kluwer Health, Lippincott Williams \& Wilkins.

Pong, S.-1., \& Zeiser, K. L. (2012). Student engagement, school climate, and academic achievement of immigrants' children. In C. C. T. García \& A. K. Marks (Eds.), The immigrant paradox in children and adolescents: Is becoming American a developmental risk? (pp. 209-232). Washington, D.C.: American Psychological Association.

Portes, A., Guarnizo, L. E., \& Landolt, P. (1999). The study of transnationalism: Pitfalls and promise of an emergent research field. Ethnic and Racial Studies, 22(2), 217-237. doi: 10.1080/014198799329468

Portes, A., Kyle, D., \& Eaton, W. W. (1992). Mental illness and help-seeking behavior among Mariel Cuban and Haitian refugees in South Florida. Journal of Health and Social Behavior, 283-298.

Postelnicu, C. (2013). Interdependencies between migration and globalization. Review of Economic Studies and Research Virgil Madgearu, 6(2), 115-136.

Prey, R. (2011). Different takes: Migrant world television and multiculturalism in South Korea. Global Media Journal, 4(1), 109-125.

Price, E. L., Bereknyei, S., Kuby, A., Levinson, W., \& Braddock, C. H. (2012). New elements for informed decision making: A qualitative study of older adults' views. Patient Education and Counseling, 86(3), 335-341. doi:

10.1016/j.pec.2011.06.006

Prior, L., Chun, P. L., \& Huat, S. B. (2000). Beliefs and accounts of illness. Views from two Cantonese-speaking communities in England. Sociology of Health \& Illness, 22(6), 815-839. doi: 10.1111/1467-9566.00232

Quin, P. (2009). New Zealand health system reforms Parliamentary Library Research Paper 09/03. Wellington: New Zealand Parliament.

Rådestad, I., Westerberg, A., Ekholm, A., Davidsson-Bremborg, A., \& Erlandsson, K. (2011). Evaluation of care after stillbirth in sweden based on mothers' gratitude. British Journal of Midwifery, 19(10), 646-652. 
Rambaree, K. (2007). Bringing rigour in qualitative social research: The use of a CAQDAS. UoM Research Journal, 13. Retrieved from: http://www.ajol.info/index.php/umrj/article/view/130883/120461

Rashid, W. E., \& Jusoff, H. K. (2009). Service quality in health care setting. International Journal of Health Care Quality Assurance, 22(5), 471-482. doi: http://dx.doi.org/10.1108/09526860910975580

Ravanera, Z., \& Fernando, R. (2009). Social integration of young Canadians: What do types of attachment tell us? PSC Discussion Papers Series, 23(5), 1.

Ravenstein, E. G. (1885). The laws of migration. Journal of the Statistical Society of London, 167-235.

Richards, K. C., Campenni, C. E., \& Muse-Burke, J. L. (2010). Self-care and well-being in mental health professionals: The mediating effects of self-awareness and mindfulness. Journal of Mental Health Counseling, 32(3), 247.

Rickert, J. (2012). Patient-centered care: What it means and how to get there. Retrieved from Health Affairs Blog website: http://healthaffairs.org/blog/2012/01/24/patient-centered-care-what-it-meansand-how-to-get-there/

Ro, A. (2014). The longer you stay, the worse your health? A critical review of the negative acculturation theory among Asian immigrants. International Journal of Environmental Research and Public Health, 11(8), 8038-8057. doi: 10.3390/ijerph110808038

Robbins, D. L., \& Galperin, B. L. (2010). Constructive deviance: Striving toward organizational change in healthcare. Journal of Management and Marketing Research, 5, 1-11.

Roderick, C. (2009). Learning classic grounded theory: An account of the journey and advice for new researchers. Grounded Theory Review, 8(2), 49-63.

Rogers, C. R. (1979). The foundations of the person-centered approach. Education, $100(2), 98-107$.

Rogers, C. R., \& Koch, S. (1959). A theory of therapy, personality, and interpersonal relationships: As developed in the client-centered framework. In S. Koch (Ed.), Psychology: A study of a science (Vol. 3, pp. 184-256). New York: McGrawHill. 
Rolfe, G. (2006). Validity, trustworthiness and rigour: Quality and the idea of qualitative research. Journal of Advanced Nursing, 53(3), 304-310.

Rubalcava, L. N., Teruel, G. M., Thomas, D., \& Goldman, N. (2008). The healthy migrant effect: New findings from the Mexican family life survey. American Journal of Public Health, 98(1), 78-84.

Rudmin, F. (2003). Critical history of the acculturation psychology of assimilation, separation, integration, and marginalization. Review of General Psychology, $7(1), 3$.

Ruzek, N. A., Nguyen, D. Q., \& Herzog, D. C. (2011). Acculturation, enculturation, psychological distress and help-seeking preferences among Asian American college students. Asian American Journal of Psychology, 2(3), 181-196. doi: $10.1037 / \mathrm{a} 0024302$

Ryder, A. G., Alden, L. E., \& Paulhus, D. L. (2000). Is acculturation unidimensional or bidimensional? A head-to-head comparison in the prediction of personality, selfidentity, and adjustment. Journal of Personality and Social Psychology, 79(1), 49.

Sajid, M., \& Baig, M. (2007). Quality of health care: An absolute necessity for public satisfaction. International Journal of Health Care Quality Assurance, 20(6), 545-548.

Salant, T., \& Lauderdale, D. S. (2003). Measuring culture: A critical review of acculturation and health in Asian immigrant populations. Social Science \& Medicine, 57(1), 71-90. doi: 10.1016/S0277-9536(02)00300-3

Saldana, J. (2009). The coding manual for qualitative researchers (1st ed.). London, Thousand Oaks: SAGE Publications.

Sam, D. L., \& Berry, J. W. (2010). Acculturation: When individuals and groups of different cultural backgrounds meet. Perspectives on Psychological Science, 5(4), 472-481. doi: 10.1177/1745691610373075

Sanou, D., O’Reilly, E., Ngnie-Teta, I., Batal, M., Mondain, N., Andrew, C., . . . Bourgeault, I. (2014). Acculturation and nutritional health of immigrants in Canada: A scoping review. Journal of Immigrant and Minority Health, 16(1), 24-34. doi: 10.1007/s10903-013-9823-7 
Schatzman, L. (1991). Dimensional analysis: Notes on an alternative approach to the grounding of theory in qualitative research. In D. R. Maines (Ed.), Social organization and social process: Essays in honor of anselm Strauss (pp. 303314). New York: Aldine de Gruyter.

Scheppers, E., van Dongen, E., Dekker, J., Geertzen, J., \& Dekker, J. (2006). Potential barriers to the use of health services among ethnic minorities: A review. Family Practice, 23(3), 325-348. doi: 10.1093/fampra/cmi113

Schimmele, C., \& Wu, Z. (2015). The new immigration and ethnic identity. Population Change and Lifecourse Strategic Knowledge Cluster Discussion Paper Series/Un Réseau stratégique de connaissances Changements de population et parcours de vie Document de travail, 3(1), 1.

Schneidewind, D. K. (2016). Economic miracle market South Korea: A blueprint for economic growth in developing nations. Singapore: Springer.

Schoen, C., Osborn, R., How, S. K., Doty, M. M., \& Peugh, J. (2009). In chronic condition: Experiences of patients with complex health care needs, in eight countries, 2008. Health Affairs, 28(1), w1-w16.

Scholte, J. A. (2005). Globalization, second edition: A critical introduction (2nd ed.). New York: Palgrave Macmillan.

Schouten, B. C., \& Meeuwesen, L. (2006). Cultural differences in medical communication: A review of the literature. Patient Education and Counseling, 64(1), 21-34.

Schwandt, T. A. (2007). The SAGE dictionary of qualitative inquiry (3rd ed.). Thousand Oaks: SAGE Publications, Inc.

Schwartz, S. J., Montgomery, M. J., \& Briones, E. (2006). The role of identity in acculturation among immigrant people: Theoretical propositions, empirical questions, and applied recommendations. Human Development, 49(1), 1-30. doi: $10.1159 / 000090300$

Schwartz, S. J., \& Unger, J. B. (2010). Biculturalism and context: What is biculturalism, and when is it adaptive?: Commentary on Mistry and Wu. Human Development, 53(1), 26-32. doi: 10.1159/000268137 
Schwartz, S. J., Unger, J. B., Zamboanga, B. L., \& Szapocznik, J. (2010). Rethinking the concept of acculturation: Implications for theory and research. The American Psychologist, 65(4), 237-251. doi: 10.1037/a0019330

Schwartz, S. J., \& Zamboanga, B. L. (2008). Testing Berry's model of acculturation: A confirmatory latent class approach. Cultural Diversity and Ethnic Minority Psychology, 14(4), 275-285. doi: 10.1037/a0012818

Scragg, R. (2010). Asian health in Aotearoa in 2006 - 2007: Trends since 2002-2003. Auckland: Northern DHB Support Agency.

Senić, V., \& Marinković, V. (2013). Patient care, satisfaction and service quality in health care. International Journal of Consumer Studies, 37(3), 312-319.

Shi, L., \& Stevens, G. D. (2005). Vulnerability and unmet health care needs. Journal of General Internal Medicine, 20(2), 148-154.

Shin, C.-N., \& Lach, H. W. (2014). Acculturation and health of Korean American adults. Journal of Transcultural Nursing, 25(3), 273-280. doi: $10.1177 / 1043659614523454$

Shin, H., Song, H., Kim, J., \& Probst, J. C. (2005). Insurance, acculturation, and health service utilization among Korean-Americans. Journal of Immigrant Health, $7(2), 65-74$.

Shortus, T., Kemp, L., McKenzie, S., \& Harris, M. (2013). 'Managing patient involvement': Provider perspectives on diabetes decision-making. Health Expectations, 16(2), 189-198.

Smith, J. E. (2010). Biculturalism and multiculturalism: Competing tensions in visual arts education in Aotearoa-New Zealand. International Journal of Multicultural Education, 12(2), 1-18.

Simmons, O. E. (2011). Why classic grounded theory. In V. B. Martin \& A. Gynnild (Eds.), Grounded theory : The philosophy, method, and work of Barney Glaser (pp. 15-30). Boca Raton, Florida: BrownWalker Press.

Singham, M. (2006). Multiculturalism in New Zealand - the need for a new paradigm. Aotearoa Ethnic Network Journa, 1(1), 33-37. http://www.aen.org.nz/journal/1/1/AENJ.1.1.Singham.pdf 
Sixma, H. J., Kerssens, J. J., Campen, C. v., \& Peters, L. (1998). Quality of care from the patients' perspective: From theoretical concept to a new measuring instrument. Health Expectations, 1(2), 82-95.

Skrentny, J. D., Chan, S., Fox, J., \& Kim, D. (2007). Defining nations in Asia and Europe: A comparative analysis of ethnic return migration policy. International Migration Review, 41(4), 793-825. doi: 10.1111/j.1747-7379.2007.00100.x

Śleziak, T. (2013). The role of confucianism in contemporary South Korean society. Rocznik Orientalistyczny/Yearbook of Oriental Studies, 1, 27-46. Retrieved from: http://hdl.handle.net/11089/3401

Smith, T. B., \& Silva, L. (2011). Ethnic identity and personal well-being of people of color: A meta-analysis. Journal of Counseling Psychology, 58(1), 42.

Smokowski, P. R., David-Ferdon, C., \& Bacallao, M. L. (2009). Acculturation and adolescent health: Moving the field forward. The Journal of Primary Prevention, 30(3-4), 209-214.

Son, J. (2013). Assimilation and health service utilization of Korean immigrant women. Qualitative Health Research. doi: 10.1177/1049732313507142

Song, C. (2013). Ethnic entrepreneurship of Korean New Zealanders: Restaurant business as self-employment practice. New Zealand Journal of Asian Studies, 15(2), 94-109.

Song, Y. J. (2009). The South Korean health care system. International Medical Community, 52(3), 206-209.

St. Louis, G. R., \& Liem, J. H. (2005). Ego identity, ethnic identity, and the psychosocial well-being of ethnic minority and majority college students. Identity, 5(3), 227-246.

Statistics Canada. (2015). Classification of visible minority. Ottawa (ON). Retrieved from http://www.statcan.gc.ca/eng/concepts/definitions/minority01a (accessed 2016 Feb.04).

Statistics Korea. (2015). 세계와 한국의 인구현황 및 전망 [World and Korean population and prospects]. Retrieved from http://kostat.go.kr.

Statistics New Zealand. (2006). Quick stats about culture and identity, 2006 Census. Retrieved from 
http://www.stats.govt.nz/Census/2006CensusHomePage/QuickStats/quickstatsabout-a-subject/culture-and-identity.aspx\#.

Statistics New Zealand. (2007). Growing ethnic diversity in New Zealand and its implications for measuring differentials in fertility and mortality. Wellington: Author.

Statistics New Zealand. (2013). New Zealand has more ethnicities than the world has countries. Wellington: Author. Retrieved from http://www.stats.govt.nz/Census/2013-census/data-tables/totals-by-topicmr1.aspx

Statistics New Zealand. (2014a). 2013 Census ethnic group profiles: Korean. Retrieved from http://www.stats.govt.nz/Census/2013-census/profile-and-summaryreports/ethnic-profiles.aspx?

Statistics New Zealand. (2014b). 2013 Census quickstats about culture and identity. Wellington: Author. Retrieved from http://www.stats.govt.nz/Census/2013census/profile-and-summary-reports/quickstats-culture-identity.aspx.

Statistics New Zealand. (2014c). 2013 Census quickstats about income. Retrieved from http://www.stats.govt.nz/Census/2013-census/profile-and-summaryreports/quickstats-income.aspx.

Stephenson, R., \& Hennink, M. (2004). Barriers to family planning service use among the urban poor in Pakistan. Opportunities and choices working paper No. 2. Retrieved from http://r4d.dfid.gov.uk/PDF/Outputs/HOppsWork2.pdf

Strauss, A., \& Corbin, J. (1994). Grounded theory methodology. In N. K. Denzin \& Y. S. Lincoln (Eds.), Handbook of qualitative research (1st ed., pp. 273-285). London: Sage Publications.

Strauss, A., \& Corbin, J. (1998). Basics of qualitative research: Techniques and procedures for developing grounded theory (2nd ed.). Thousand Oaks, London, New Delhi: SAGE Publications.

Suårez-Orozco, C., Todorova, I. L. G., \& Louie, J. (2002). Making up for lost time: The experience of separation and reunification among immigrant families. Family Process, 41(4), 625-643. doi: 10.1111/j.1545-5300.2002.00625.x

Sullivan, K. R., \& Ebrahim, G. J. (1995). Mother and child health: Research methods (2 ed.). London: Book Aid. 
Suurmond, J., Uiters, E., de Bruijne, M. C., Stronks, K., \& Essink-Bot, M. L. (2011). Negative health care experiences of immigrant patients: A qualitative study. BMC Health Serv Res, 11, 10. doi: 10.1186/1472-6963-11-10

Tam Ashing, K., Padilla, G., Tejero, J., \& Kagawa-Singer, M. (2003). Understanding the breast cancer experience of Asian American women. Psycho-Oncology, 12(1), 38-58.

Tan, J. (2010). Grounded theory in practice: Issues and discussion for new qualitative researchers. Journal of Documentation, 66(1), 93-112.

Tarozzi, M. (2011). On translating grounded theory when translating is doing. In V. B. Martin \& A. Gynnild (Eds.), Grounded theory : The philosophy, method, and work of Barney Glaser (pp. 161-174). Boca Raton, Florida: BrownWalker Press.

Temple, B., \& Young, A. (2004). Qualitative research and translation dilemmas. Qualitative Research, 4(2), 161-178.

Thieme, S. (2006). Social networks and migration: Far West Nepalese labour migrants in Delhi (Vol. 7). New Brunswick, London: Transaction Publishers.

Thieme, S. (2007). A brief overview of theories of international migration. Retrieved from: http://www.glopp.ch/C1/en/multimedia/C1_pdf1.pdf

Thorns, D. C., Fairbairn-Dunlop, T. P., \& Du Plessis, R. (2010). Biculturalism, cultural diversity and globalisation: Issues for Aotearoa New Zealand. Korean Social Science Journal, XXVII, 1, 93-122.

Tse, S., Wong, G. H., Hong, C., \& Rasalingam, N. (2013). Asian people in New Zealand. In I. St George (Ed.), Cole's medical practice in New Zealand, 12th edition (pp. 73-82). Wellington: Medical Council of New Zealand.

Tucker, J. L., \& Adams, S. R. (2001). Incorporating patients' assessments of satisfaction and quality: An integrative model of patients' evaluations of their care. Managing Service Quality: An International Journal, 11(4), 272-287. doi: 10.1108/EUM0000000005611

Turner, L. (2007). 'First world health care at third world prices': Globalization, bioethics and medical tourism. Biosocieties, 2(3), 303-325. doi: $10.1017 / \mathrm{S} 1745855207005765$

Turney, K., \& Kao, G. (2012). Behavioral outcomes in early childhood: Immigrant paradox or disadvantage? In C. G. Coll \& A. K. Marks (Eds.), The immigrant 
paradox in children and adolescents: Is becoming American a developmental risk? (pp. 79-107). Washington, DC: American Psychological Association.

Uchudi, J. M. (2001). Covariates of child mortality in mali: Does the health-seeking behaviour of the mother matter? Journal of Biosocial Science, 33(1), 33-54.

Umaña-Taylor, A. J. (2004). Ethnic identity and self-esteem: Examining the role of social context. Journal of Adolescence, 27(2), 139-146. doi: http://dx.doi.org/10.1016/j.adolescence.2003.11.006

Umaña-Taylor, A. J., Wong, J. J., Gonzales, N. A., \& Dumka, L. E. (2012). Ethnic identity and gender as moderators of the association between discrimination and academic adjustment among Mexican-origin adolescents. Journal of Adolescence, 35(4), 773-786. doi: http://dx.doi.org/10.1016/j.adolescence.2011.11.003

UNHCR. (2015). The sea route to Europe: The mediterranean passage in the age of refugees. Retrieved from: http://www.unhcr.org/5592bd059.pdf

UNHCR. (2016, January 19). Registered Syrian refugees. from http://data.unhcr.org/syrianrefugees/regional.php

United Nations Population Division. (2013). Trends in international migrant stock: The 2013 revision-migrants by destination and origin. New York: UN Department of Economic and Social Affairs Retrieved from http://www.un.org/en/development/desa/population/publications/pdf/migration/ migrant-stock-origin-2013.pdf.

Van Hear, N. (2010). Theories of migration and social change. Journal of Ethnic and Migration Studies, 36(10), 1531-1536.

van Nes, F., Abma, T., Jonsson, H., \& Deeg, D. (2010). Language differences in qualitative research: Is meaning lost in translation? European Journal of Ageing, 7(4), 313-316. doi: 10.1007/s10433-010-0168-y

Vang, Z., Sigouin, J., Flenon, A., \& Gagnon, A. (2015). The Healthy Immigrant Effect in Canada: A systematic review. Population Change and Lifecourse Strategic Knowledge Cluster Discussion Paper Series/Un Réseau stratégique de connaissances Changements de population et parcours de vie Document de travail, 3(1), 4. 
Vertovec, S. (2004). Migrant transnationalism and modes of transformation. The International Migration Review, 38(3), 970-1001.

Vertovec, S. (2007). Circular migration: The way forward in global policy? Working Paper No. 4. International Migration Institute, University of Oxford. UK. Retrieved from http://www.imi.ox.ac.uk/pdfs/wp/wp-04-07.pdf

Vieru, A. M. (2013). The process of migration and integration: A theoretical inquiry. Social Research Reports, 24, 7-15.

Wang, L., \& Kwak, M.-J. (2014). Immigration, barriers to healthcare and transnational ties: A case study of South Korean immigrants in Toronto, Canada. Social Science \& Medicine. doi: 10.1016/j.socscimed.2014.11.039

Wang, Z. (2010). Self-globalisation - a new concept in the push-and-pull theory: A study on Chinese self-funded master students. Paper presented at the Education and Citizenship in a Globalising World, London. http://www.ioe.ac.uk/about/documents/About_Overview/Wang_Z.pdf

Ward, H., Mertens, T. E., \& Thomas, C. (1997). Health seeking behaviour and the control of sexually transmitted disease. Health Policy and Planning, 12(1), 1928.

Weingart, S. N., Pagovich, O., Sands, D. Z., Li, J. M., Aronson, M. D., Davis, R. B., . . Phillips, R. S. (2005). What can hospitalized patients tell us about adverse events? Learning from patient-reported incidents. Journal of General Internal Medicine, 20(9), 830-836.

Wen, L. (2013). Should you be a "patient" or "healthcare consumer"? Psychology Today. Retrieved from: https://www.psychologytoday.com/blog/the-doctor-islistening/201305/should-you-be-patient-or-healthcare-consumer

Westbrook, R. A. (1981). Sources of consumer satisfaction with retail outlets. Journal of Retailing, 57(3), 68-85.

WHO. (1983). Health education in self-care: Possibilities and limitations. Geneva, Switzerland. http://apps.who.int/iris/bitstream/10665/70092/1/HED_84.1.pdf

WHO, \& Ministry of Health. (2012). Health service delivery profile New Zealand. Retrieved from http://www.wpro.who.int/health_services/service_delivery_profile_new_zealand .pdf. 
WHO, \& Ministry of Health and Welfare. (2012). Health service delivery profile, Republic of Korea, 2012 Retrieved from http://www.wpro.who.int/health_services/service_delivery_profile_republic_of_ korea.pdf?ua=1.

Williams, M. T., Chapman, L. K., Wong, J., \& Turkheimer, E. (2012). The role of ethnic identity in symptoms of anxiety and depression in African Americans. Psychiatry Research, 199(1), 31-36. doi: 10.1016/j.psychres.2012.03.049

Willig, C. (2013). Introducing qualitative research in psychology: Adventures in theory and method (3rd ed.). Buckingham, Philadelphia: Open University Press.

Wong, S., Dixon, L. B., Gilbride, J., Kwan, T., \& Stein, R. (2013). Measures of acculturation are associated with cardiovascular disease risk factors, dietary intakes, and physical activity in older Chinese Americans in New York City. Journal of Immigrant and Minority Health, 15(3), 560-568. doi: 10.1007/s10903-012-9669-4

World Vision NZ. (2012, 2012). About world vision. from http://www.worldvision.org.nz/about-us.aspx

Xhaferaj, E. (2014). Migration theories: A comparative approach from neo-classical to contemporary perspectives. Retrieved from PECOB: Portal on Central Eastern and Balkan Europe by IECOB \& AIS website: http://www.pecob.eu/Migrationtheories-comparative-approach-neo-classical-contemporary-perspectives

Yoon, H. K., \& Yim, S. H. (1997). Nyujilandu okulandu hankukin sengup punsosk [an analysis of occupations of Korean immigrants in Auckland, New Zealand]. Journal of the Korean Geographical Society, 32(4), 491-510.

Zaner, R. M. (2015). A critical examination of ethics in health care and biomedical research: Voices and visions. Heidelberg, Dordrecht, New York, London: Springer International Publishing.

Zeithaml, V. A. (2000). Service quality, profitability, and the economic worth of customers: What we know and what we need to learn. Journal of the Academy of Marketing Science, 28(1), 67-85.

Zhou, L. (2009). Health needs assessment for Asian people in Waitemata. Auckland: Waitemata District Health Board. 
Zimmerman, C., Kiss, L., \& Hossain, M. (2011). Migration and health: A framework for 21st century policy-making. PLoS Med, 8(5), e1001034.

Zohry, A. (2005). Interrelationships between internal and international migration in Egypt: A pilot study. Research Reports Series. Development Research Center on Migration, Globalization, and Poverty, University of Sussex. Brighton, UK. Retrieved from http://r4d.dfid.gov.uk/PDF/Outputs/MigrationGlobPov/AymanReport.pdf 